\title{
Inferring Ocean Circulation during the Last Glacial Maximum and Last Deglaciation Using Data and Models
}

\author{
by \\ Daniel Edward Amrhein \\ B.A., Columbia University (2009) \\ M.S., Massachusetts Institute of Technology / Woods Hole Oceanographic Institution \\ Joint Program in Oceanography (2014)
}

Submitted in partial fulfillment of the requirements for the degree of Doctor of Philosophy

at the

\begin{abstract}
MASSACHUSETTS INSTITUTE OF TECHNOLOGY
and the

WOODS HOLE OCEANOGRAPHIC INSTITUTION
\end{abstract}

September 2016

(C) 2016 Daniel Edward Amrhein. All rights reserved.

The author hereby grants to MIT and WHOI permission to reproduce and to distribute publicly paper and electronic copies of this thesis document in whole or in part in any medium now known or hereafter created.

Signature of author

Joint Program in Oceanography - Massachusetts Institute of Technology/

Woods Hole Oceanographic Institution

15 August 2016

Certified by

Carl Wunsch

Cecil and Ida Green Professor of Physical Oceanography, Emeritus

Massachusetts Institute of Technology

Thesis Advisor

Accepted by

Larry Pratt

Senior Scientist, Woods Hole Oceanographic Institution Chair, Joint Committee on Physical Oceanography 


\title{
Inferring Ocean Circulation During the Last Glacial Maximum and Last Deglaciation Using Data and Models
}

by

\author{
Daniel Edward Amrhein
}

\begin{abstract}
Submitted to the Joint Program in Oceanography,
Massachusetts Institute of Technology and the Woods Hole Oceanographic Institution on August 15, 2016, in partial fulfillment of the requirements for the degree of

Doctor of Philosophy
\end{abstract}

\begin{abstract}
Since the Last Glacial Maximum (LGM, 20,000 years ago) air temperatures warmed, sea level rose roughly 130 meters, and atmospheric concentrations of carbon dioxide increased. This thesis combines global models and paleoceanographic observations to constrain the ocean's role in storing and transporting heat, salt, and other tracers during this time, with implications for understanding how the modern ocean works and how it might change in the future.
\end{abstract}

- By combining a kinematic ocean model with "upstream" and "downstream" deglacial oxygen isotope time series from benthic and planktonic foraminifera, I show that the data are in agreement with the modern circulation, quantify their power to infer circulation changes, and propose new data locations.

- An ocean general circulation model (the MITgcm) constrained to fit LGM sea surface temperature proxy observations reveals colder ocean temperatures, greater sea ice extent, and changes in ocean mixed layer depth, and suggests that some features in the data are not robust.

- A sensitivity analysis in the MITgcm demonstrates that changes in winds or in ocean turbulent transport can explain the hypothesis that the boundary between deep Atlantic waters originating from Northern and Southern Hemispheres was shallower at the LGM than it is today.

Thesis supervisor: Carl Wunsch

Title: Cecil and Ida Green Professor of Physical Oceanography, Emeritus 


\section{Acknowledgements}

I am grateful for the support and friendship of many people during my time in the Joint Program. Carl has been an exceptional advisor, always enthusiastic about the work and full of good questions. Numerous conversations with committee members (Olivier Marchal, Patrick Heimbach, David McGee, and Mick Follows) were important for shaping the directions of the research. Jean-Michel Campin, An Nguyen, and Gael Forget played the Virgils to my Dante as I burrowed my way into the MITgcm. Jon Lauderdale contributed an MITgcm setup that was very helpful in the work in Chapter 4. Other researchers at MIT and WHOI (Jake Gebbie, Ken Brink, Jeff Scott, Oliver Jahn...) provided assistance at various critical junctures. Many thanks goes to the saintly people in EAPS HQ, APO, PO, and PAOC for making the administrative necessities of graduate school minimally onerous for students.

Finally, I have been very lucky to have the support of my family and the company of a noble troupe of diversely talented and debauched individuals in my graduate school cohort.

Most oxygen isotope records shown in Chapter 2 can be obtained through the PANGAEA (pangaea.de/) and NOAA NCDC (www.ncdc.noaa.gov/data-access/paleoclimatology-data/) online archives; the remainder were communicated personally by Delia Oppo, Jerry McManus, Claire Waelbroeck, Luke Skinner, and Simon Jung. For access to MATLAB codes used in analyses and figure making, objectively mapped data, and record ODP980, please contact the corresponding author. Thanks goes to Ed Boyle, Lloyd Keigwin, Elisabeth Michel, Delia Oppo, David Thornalley, and Claire Waelbroeck for helpful discussions about sediment core records and their interpretation. Thoughtful comments from François Primeau and an anonymous reviewer greatly improved the associated manuscript.

For their roles in producing, coordinating, and making available the CMIP5 model output, we acknowledge the CCSM climate modeling group, the World Climate Research Programme's (WCRP) Working Group on Coupled Modelling (WGCM), and the Global Organization for Earth System Science Portals (GO-ESSP).

Support for this work came from an MIT Presidential Fellowship, an NSF Graduate Research Fellowship, and grants NASA NNX12AJ93G - Gravity data for ocean circulation and climate studies, NSF OCE-0961713 - Collaborative Research: The Physics and Statistics of Global Sea Level Change, NSF OCE-1060735 - Collaborative Research: Beyond the Instrumental Record - the Ocean Circulation at the last Glacial maximum and the deglacial sequence, and NASA NNX08AR33G - Application of Satellite Altimetry Gravity Winds and in Situ Data to Problems of the Ocean Circulation.

Permission to reprint figures from previous publications was granted through an MIT license; for more information please see https://libraries.mit.edu/scholarly/publishing/usingfigures-from-major-publishers-in-new-scholarly-articles/. 


\section{Contents}

\begin{tabular}{llr}
\hline 1 & Introduction & 19
\end{tabular}

2 Inferring surface water equilibrium calcite $\delta^{18} \mathrm{O}$ during the last deglacial period from benthic foraminiferal records: Implications for ocean circulation $\quad \mathbf{2 5}$

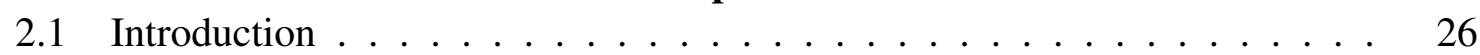

2.2 Benthic and planktonic $\delta^{18} \mathrm{O}_{c}$ records . . . . . . . . . . . . . . . . 29

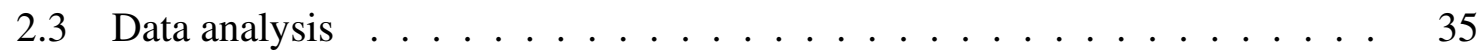

2.3.1 Relating benthic proxies to ML conditions . . . . . . . . . . . . 35

2.3.2 $\quad$ Solution method for benthic-derived $\mathrm{ML} \delta^{18} \mathrm{O}_{c} \ldots \ldots . \ldots 38$

2.3 .3 Relating planktonic proxies to ML conditions . . . . . . . . . . 43

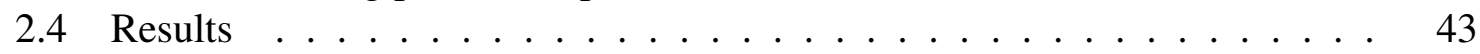

$2.4 .1 \quad \mathrm{ML} \delta^{18} \mathrm{O}_{c}$ inferred from benthic data . . . . . . . . . 43

2.4.2 Comparison of ML $\delta^{18} \mathrm{O}_{c}$ estimates from benthic and planktonic data 48

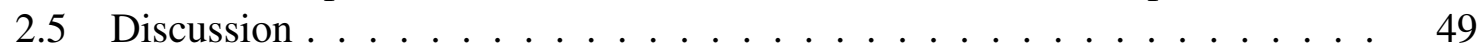

2.6 Conclusions . . . . . . . . . . . . . . . . . . . . . . . . . . . . . . . . . . . . . . . . . . .

2.7 Appendices $\ldots \ldots \ldots \ldots \ldots \ldots$

2.7 .1 Statement of the inverse problem . . . . . . . . . . 56

2.7.2 Computation of regional averages and uncertainties . . . . . . . . 58

2.7 .3 Inference of $\mathrm{ML} \delta^{18} \mathrm{O}_{c}$ conditions in a four-box model . . . . . . 59

3 GOSE: A Glacial Ocean State Estimate constrained by LGM near-sea surface temperature proxies $\quad 63$

3.1 Introduction . . . . . . . . . . . . . . . . . . . 63

3.2 Background . . . . . . . . . . . . . . . . . 66

3.2.1 Observations and simulations of near-sea surface temperature at the

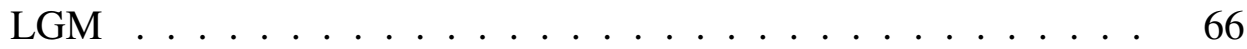

3.2.2 Previous inverse studies of the LGM constrained by MARGO obser-

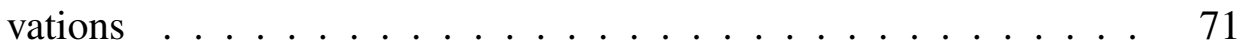

3.2.3 State estimation using the method of Lagrange multipliers . . . . . 72

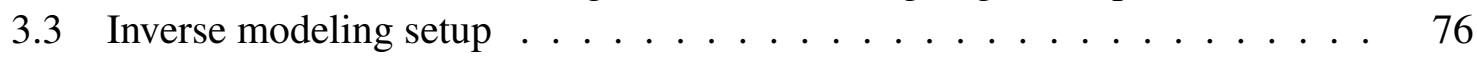

3.3 .1 The MITgcm . . . . . . . . . . . . . . . . . 76

3.3.2 First guess control variables and prior covariances . . . . . . . 78

3.3 .3 Construction of the cost function . . . . . . . . . . . . 83 
8.4 Results. . . . . . . . . . . . . . . . . . . . . . . . . . . . . . . . . . . . . . . 84

3.4.1 A quasi-equilibrated MITgcm simulation forced by CCSM4 bound-

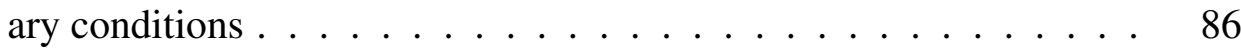

3.4 .2 Fitting the model to data $\ldots \ldots \ldots \ldots \ldots$. . . . . . . 90

$9.4 .3 \quad$ Analysis of the state estimate. . . . . . . . . . . . . . . . . . . 96

3.4.3.1 Near-sea surface temperatures . . . . . . . . . . . . . . . 96

3.4.3.2 Barotropic streamfunction . . . . . . . . . . . . . 97

3.4.3.3 AMOC and meridional heat transport . . . . . . . . . . 97

3.4.3.4 Northern Hemisphere sea ice . . . . . . . . . . . . . . 100

3.4.3.5 Mixed layer depth . . . . . . . . . . . . . . . . . . . 103

3.4.4 Evaluating the fit of $\overline{\mathbf{x}}_{M A R G O}$ to LGM $\delta^{18} \mathrm{O}$ observations $\ldots \ldots$

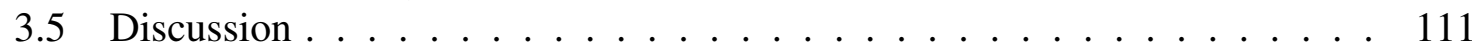

3.6 Conclusions and future work . . . . . . . . . . . . . . . . . . . . . . 118

4 Ocean state estimation from passive tracer observations 121

4.1 Introduction . . . . . . . . . . . . . . . . . . . . . . . 121

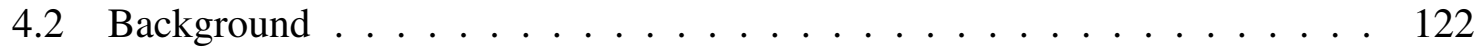

4.2 .1 Inferences from tracers at the LGM . . . . . . . . . . . 122

4.2.2 Challenges of seasonally quasi-steady ocean state estimation using passive tracer observations $\ldots \ldots \ldots \ldots \ldots \ldots$

4.3 Procedures for accelerating passive tracer equilibration for use in state esti-

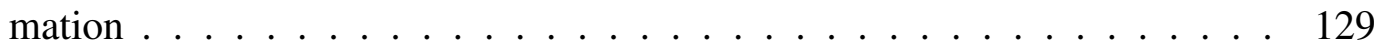

$4.3 .1 \quad$ Asynchronous time stepping . . . . . . . . . . . . . . . . . . . 129

4.3.2 Jacobi preconditioning to accelerate tracer convergence and provide a scaling for vertically asynchronous time stepping $\ldots \ldots \ldots . . \ldots 131$

4.3.2.1 Development. . . . . . . . . . . . . . 131

4.3.2.2 Demonstration in a four-box model . . . . . . . . . . . . 136

$4.3 .3 \quad$ Extrapolating tracer values using a "helper" tracer $\ldots \ldots \ldots \ldots$

$4.3 .3 .1 \quad$ Development . . . . . . . . . . . . . . . . . . . . 139

4 4.3.3.2 Demonstration in a four-box model . . . . . . . . . . . 142

4.3.3.3 Extensions and future directions . . . . . . . . . . . . 144

4.4 Evaluating tracer equilibration procedures in the MITgcm . . . . . . . . . 146

4.5 Why might Antarctic Bottom Water shoal in the Atlantic Ocean? . . . . . 153

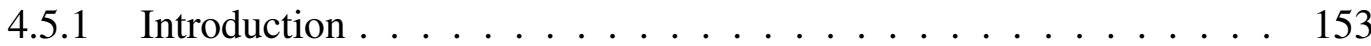

4.5 .2 Methods . . . . . . . . . . . . . . . . . 153

4.5.2.1 MITgcm configuration. . . . . . . . . . . . . 153

4.5.2.2 Setup of the inverse problem . . . . . . . . . . . . 154

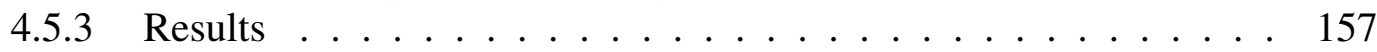

4.5 .4 Discussion $\ldots \ldots \ldots \ldots \ldots \ldots$

4.6 Conclusions $\ldots \ldots \ldots \ldots \ldots$

5 Conclusions and future work $\quad 169$ 


\section{List of Figures}

2.1 Locations of the eight benthic (blue, with labels) and 13 planktonic (red squares) records of $\delta^{18} \mathrm{O}_{c}$ used in this study. Locations and names of planktonic records are listed in Table $2.3 ! \ldots \ldots$. . . . . . . . . . . 30

2.2 Eight benthic foraminiferal $\delta^{18} \mathrm{O}_{c}$ anomaly records are used to infer ML conditions. Blue dots show the published data, the solid black line their objectively mapped values, and the grey shading the objectively mapped uncertainty. Vertical grid spacing is $1 \% 0$ and record order is arbitrary. Red lines show the reconstructed data (see text) derived from the $\mathrm{ML} \delta^{18} \mathrm{O}_{c}$ solution obtained in Section|2.4|with the small effect due to initial conditions added

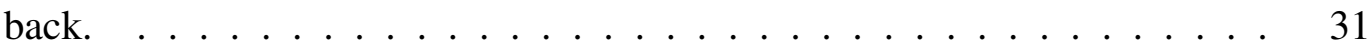

2.3 Adjoint Green's functions for NA3146 (red, Iberian Margin) and EP3210 (blue, Eastern Equatorial Pacific) computed using the GH12 model (see text). The adjoint Green's functions may be interpreted as follows. In the case when ML $\delta^{18} \mathrm{O}_{c}$ conditions are a delta function in the $i^{\text {th }}$ ML grid box at $t=0$ (that is, $q_{i}(t)=1 \%$ at $t=0$, and $q(t)=0 \%$ at all other places and times), the signal at each deep core site as the model is integrated forward in time is equal to the time trajectory in (b), scaled by the fraction indicated by circle area in the $i^{t h}$ grid box in (a). Where there are no circles, tracer communicated to core sites is very small or zero. An approximation is made in representing the adjoint Green's functions by a pair of spatial and temporal patterns (see text); over $90 \%$ of the variability is accounted for at both core

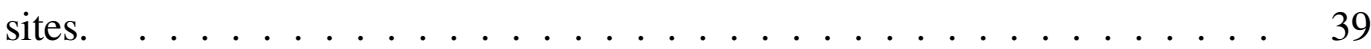

2.4 (a) Circle area centered on a grid box indicates the pointwise resolvability $r$; filled circles over land in the lower left are a legend. Grid points with no circles have resolvability $<0.01$. Lighter gray areas define regions plotted in the lower panel. Box 1 and the region south of the dotted line at $50^{\circ} \mathrm{S}$ are averaged to compute the North Atlantic and Southern Ocean time series $\tilde{m}_{N A}$ and $\tilde{m}_{S O}$, respectively (see text). (b) The benthic-derived $\mathrm{ML} \delta^{18} \mathrm{O}_{c}$ solution plotted as overlaid time series at every grid box within the five geographical regions and shaded by pointwise resolvability. In all regions, values after 6 ka lie in the nullspace and are equal to zero, as they are not constrained by benthic observations, which end at $5 \mathrm{ka}$. Note the nonlinear color bar. . . . 46 
2.5 Estimates of $\mathrm{ML} \delta^{18} \mathrm{O}_{c}$ anomalies in the North Atlantic $\left(\tilde{m}_{N A}\right)$ and Southern Oceans $\left(\tilde{m}_{S O}\right)$. The inner error bar is the standard error, the middle envelope includes the nullspace contribution when $\mathrm{ML} \delta^{18} \mathrm{O}_{c}$ is assumed hemispherically covarying, and the outer envelope includes the nullspace contribution when $\mathrm{ML} \delta^{18} \mathrm{O}_{c}$ is assumed spatially uncorrelated (see text). . . . . . . . .

2.6 Comparison of thirteen planktonic $\delta^{18} \mathrm{O}_{c}$ anomaly records (black curves) and $\delta^{18} \mathrm{O}_{c}$ anomaly records derived from benthic records (blue curves) in the ML regions overlying planktonic core sites in the Northern (a) and Southern (b) Hemispheres. Panels are ordered by latitude. Pointwise resolvabilities, $r$ (Section 2.3), and Pearson correlations, $R$, are shown to the right of each panel. Very small values of $r$ (order $10^{-5}$ ) are practically indistinguishable from zero. The vertical grid spacing is $1 \% 0 . \mathrm{ML} \delta^{18} \mathrm{O}_{c}$ in the region overlying MD03_2707 lies entirely in the null space of the inverse problem $(r=0)$ and as such is inferred to be 0 at all times. . . . . . . . . . . . . 50

2.7 Northern (a) and Southern (b) Hemisphere planktonic $\delta^{18} \mathrm{O}_{c}$ anomalies overlaid with means removed. Thick black lines are the hemispheric time means computed after smoothing and subsampling each record (see text). Grey bars give the standard deviations of the records about the mean at each time. . . 51

2.8 Comparison of spatially averaged $\mathrm{ML} \delta^{18} \mathrm{O}_{c}$ anomalies inferred from benthic records in the North Atlantic $\left(\tilde{m}_{N A}\right)$ and Southern $\left(\tilde{m}_{S O}\right)$ Oceans to averages of planktonic $\delta^{18} \mathrm{O}_{c}$ anomalies from the Northern and Southern Hemispheres $\left(\tilde{m}_{N H}^{p}\right.$ and $\tilde{m}_{S H}^{p}$, respectively). Uncertainties are the same as those described for Figures $2.5 \mid$ and $|2.7| \ldots \ldots \ldots \ldots$. . . . . . . . . . . 52

2.9 (a) Four-box tracer transport model schematic. $\delta^{18} \mathrm{O}_{c}$ values $q_{M}$ and $q_{H}$ in two ML boxes are injected into box 1 at rates $F_{M}=1 \mathrm{~Sv}$ and $F_{H}=9 \mathrm{~Sv}$. Fluid is homogenized in box 1 and flows into box 2 at a rate $F_{M}+F_{H}=10$ Sv, where it subsequently leaves the system. (b) Green's function for $q_{M}\left(q_{H}\right)$ is generated by stepping Eq. (2.26) forward in time forced by ML conditions that are zero in both boxes at all times except $q_{M}(0)=1\left(q_{H}(0)=1\right)$. (c, d) ML $\delta^{18} \mathrm{O}_{c}$ anomaly estimates and their standard error in cases without (c) and with (d) column normalization. In both cases values at 5 ka lie in the nullspace and are 0 , as they are not constrained by benthic observations. In (d), $\tilde{q}_{M}(t)$ and $\tilde{q}_{H}(t)$ are identical. All $\delta^{18} \mathrm{O}_{c}$ values have units of permil

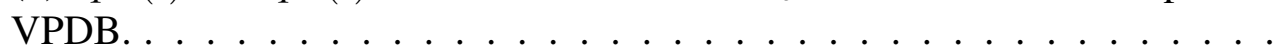

3.1 Interpolated LGM sea surface temperature anomalies from Waelbroeck et al. [2009], their Figure 2. Anomalies are computed as the difference between LGM temperature reconstructions and the World Ocean Atlas [Conkright et al.[1998]. a, July-August-September mean; b, January-February-March mean; c, annual mean. Symbols denote proxy locations. Reprinted by permission from Macmillan Publishers Ltd: Nature Geoscience, Waelbroeck

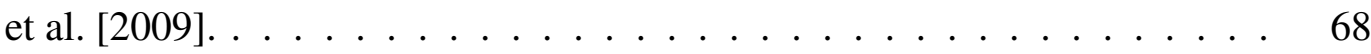


3.2 Figure 1 from Otto-Bliesner et al. [2007] showing the Atlantic Meridional Overturning Circulation (AMOC) streamfunction simulated by the PMIP2 coupled atmosphere-ocean general circulation models (GCMs) for modern (top) and LGM conditions (bottom). Units are in Sverdrups (Sv, $\left.10^{6} \mathrm{~m}^{3} \mathrm{~s}^{-1}\right)$. A modern observational range of AMOC maxima is $18 \pm(3-5) \mathrm{Sv}$ [Talley et al., 2003]. Streamfunctions in PMIP3 are different still; see Muglia and Schmittner[2015]. . . . . . . . . . . . . . . . . . . . . . . . 70

3.3 LGM bathymetry used in the state estimate in meters. . . . . . . . . . . . 77

3.4 LGM minus preindustrial quantities derived from the CCSM4 LGM PMIP3 simulation [Brady et al. $[2013] . \ldots \ldots \ldots \ldots$. . . . . . . . . . . . 81

3.5 First guess forcings constructed by adding the CCSM4 LGM minus preindustrial quantities (Figure 3.4, Brady et al. [2013]) to the ECCO v4 adjusted forcings [Forget et al. $\mid 2015 \mathrm{a}].] \ldots \ldots$. . . . . . . . . 82

3.6 MARGO $5^{\circ} \times 5^{\circ}$ gridded temperature data and uncertainties from Waelbroeck et al.[2009] interpolated to the model grid. . . . . . . . . . . . . . 85

3.7 Temperature at $5 \mathrm{~m}$ averaged over years 1992-2011 in the ECCO v4 solution (top) and over years 4800-4900 of the spin-up simulation under CCSM4 LGM atmospheric conditions (bottom). . . . . . . . . . . . . . 88

3.8 Zonal mean Atlantic meridional streamfunction plotted as a function of latitude and depth in units of Sverdrups in the ECCO v4 solution averaged over years 1992-2011 (top) and in the spin-up simulation under CCSM4 LGM atmospheric conditions averaged over years 4800-4900 (bottom). . . . . . 89

3.9 Unscaled (left, ${ }^{\circ} \mathrm{K}$ ) and scaled (right, normalized units) model-data disagreement in the initial simulation of the MITgcm. Blue (red) values indicate that the top 3 model grid boxes are cold (warm) relative to the data. Scaled misfits are computed by dividing the unscaled misfit by the data uncertainty (Figure

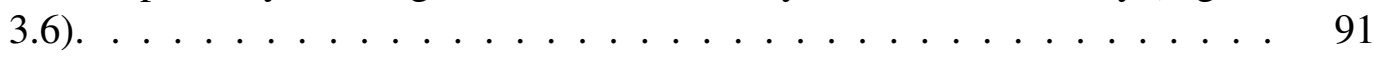

3.10 Annual model-data misfit scaled by data uncertainties after 10 iterations of the adjoint (left); as before, blue (red) values indicate that the top 3 model grid boxes are cold (warm) relative to the data. The right panel shows scaled changes in absolute misfit since iteration 0 (right); blue (red) areas indicate regions of cost reduction (increase). Results for JFM and JAS misfits are qualitatively similar. . . . . . . . . . . . . . 92

3.11 Histograms of normalized misfits to annual and boreal and austral winter average MARGO data of the $\overline{\mathbf{x}}_{M A R G O}$ solution in iterations 0,9 , and 10 . Values for iteration 9 and 10 lie very close together, and in many cases only iteration 10 (red lines) is visible. Ideally, the distribution of normalized model-data misfits would be Gaussian with zero mean and standard deviation $\sigma_{n}=1$, indicated by the grey curves. . . . . . . . . . . . . . . . 93

3.12 Annual mean control changes used to generate $\overline{\mathbf{x}}_{M A R G O}$, scaled by their prior uncertainties (units of $\sigma$; see Table 3.1). 
3.13 LGM-modern NSST temperature anomalies averaged over the top three model grid boxes (spanning 30 meters) computed as the difference between $\overline{\mathbf{x}}_{M A R G O}$ and ECCO v4 for means over July-August-September (top), January-FebruaryMarch (middle), and the annual mean (bottom). LGM temperatures are computed over the last ten years of the 100 -year $\overline{\mathbf{x}}_{M A R G O}$ simulation to avoid initial transience as the model adjusts to control changes in initial temperature and salinity. . . . . . . . . . . . . . . . . . 98

3.14 LGM (top) and LGM-modern barotropic streamfunctions plotted in Sverdrups. The modern streamfunction is computed from the ECCO v4 solution averaged over 1992-2011. . . . . . . . . . . . . . . . 99

3.15 Atlantic zonal mean streamfunctions in ECCO v4 (top, 1992-2011 mean), $\overline{\mathbf{x}}_{M A R G O}$ after 9 iterations (middle) and $\overline{\mathbf{x}}_{M A R G O}$ at the final (10th) iteration (bottom). Large changes in AMOC strength between the 9th and 10th iterations, when model-data misfit is similar, suggest that the data do not constrain this quantity well. . . . . . . . . . . . . . . 101

3.16 Heat transport in PW as a function of latitude for ECCO v4 (1992-2011 mean, top) and $\overline{\mathbf{x}}_{M A R G O}$ iterations 9 (middle) and 10 (bottom). Note the different vertical axes. . . . . . . . . . . . . . . . 102

3.17 Sea ice concentration in $\overline{\mathbf{x}}_{M A R G O}$ (left) and from modern observations [Comiso, 1999] in March (top) and September (bottom). . . . . . . . . . . . . . . . . 104

3.18 Modified figure from De Vernal et al. [2006] used in Dail [2012b] showing MARGO LGM NSST reconstructions from planktonic foraminiferal assemblages (left) and from dinoflagellate cysts (dynocysts, right) and estimates of LGM sea ice extent based on these data. In both plots, grey shading shows estimates of perennial sea ice, and the dotted lines illustrate estimated maximum winter sea ice extent. . . . . . . . . . . . . . . . . . . . 104

3.19 Annual mean control changes used to generate $\overline{\mathbf{x}}_{M A R G O}$ in the Northern Hemisphere, scaled by their prior standard deviation (Table 3.1). . . . . . . . . 105

3.20 Mean March mixed layer depth in meters computed via Kara et al. [2000] in ECCO v4 (top) and $\overline{\mathbf{x}}_{M A R G O}$ in iteration 9 (middle) and 10 (bottom). . . . . 107

$3.21 \mathrm{LGM} \delta^{18} \mathrm{O}_{c}$ measurements plotted by proxy type and core depth. . . . . . 112

3.22 Top: Glacial $\delta^{18} \mathrm{O}_{c}$ ML boundary conditions estimated using the coldest monthly average temperatures from $\overline{\mathbf{x}}_{M A R G O}$ (see text). Middle and bottom: Comparison of annual average $\delta^{18} \mathrm{O}_{c}$ derived from $\overline{\mathbf{x}}_{M A R G O}$ with a database of glacial planktonic $\delta^{18} \mathrm{O}_{c}$ measurements. The black line in the bottom panel indicates perfect model-data agreement. Error bars show the range of $\delta^{18} \mathrm{O}_{c}$ arising from seasonal variations as summarized by standard deviations of monthly climatologies at data locations. . . . . . . . . . . . . 113

3.23 Model $\delta^{18} \mathrm{O}_{c}$ averaged zonally over $20^{\circ} \mathrm{W}$ to $10^{\circ} \mathrm{W}$ (a region coincident with many benthic observations, see Figure 3.21 ) with glacial benthic values overlaid. 
3.24 Histogram of normalized misfits between the global distribution of benthic $\delta^{18} \mathrm{O}_{c}$ data (Figure 3.21) and the extrapolated equilibrium $\delta^{18} \mathrm{O}_{c}$ simulated in $\overline{\mathbf{x}}_{M A R G O}$ under surface boundary conditions shown in Figure 3.22 . Misfits are normalized by a uniform uncertainty of $0.2 \%$ following Marchal and Curry [2008] and Amrhein et al.[2015]. . . . . . . . . . . . . . . 114

4.1 Concentration at $1975 \mathrm{~m}$ after 1900 years from a globally uniform surface dye distribution advected in an offline transport simulation derived from ECCO-GODAE; figure from Wunsch and Heimbach [ [2008]. The system is far from equilibrium (where the concentration everywhere is 1) in large parts of the Indian and Pacific Oceans. . . . . . . . . . . . . . . . . . . 122

4.2 Comparison of the carbon isotope ratio of dissolved organic carbon $\delta^{13} \mathrm{C}_{\mathrm{DIC}}$ at model depth $2730 \mathrm{~m}$ (colored contours) in a 10-year state estimate from Dail [2012b] (her Figure 3-17) with modern GEOSECS $\delta^{13} \mathrm{C}_{\text {DIC }}$ observations. The modeled distribution of $\delta^{13} \mathrm{C}_{\text {DIC }}$ is patchy. $\ldots . . . . . . . .128$

4.3 Misfits between observations and model simulated tracer concentrations can be biased for disequilibrated model tracer states. Here when a tracer observation (red star) assumed to represent an equilibrated system is compared to a hypothetical grid box tracer concentration trajectory (blue line) after 100

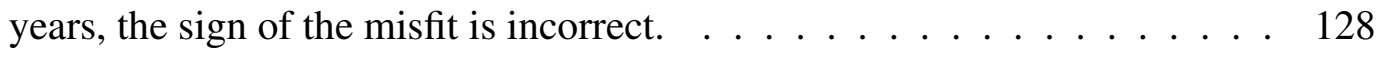

4.4 TOP: Schematic of the four-box tracer transport model. Passive tracer values $q_{M}$ and $q_{H}$ in two ML boxes are injected into box 1 at rates $F_{M}=1 \mathrm{~Sv}$ and $F_{H}=9 \mathrm{~Sv}$. Fluid is homogenized in box 1 and flows into box 2 at a rate $F_{M}+F_{H}=10 \mathrm{~Sv}$, where it subsequently leaves the system. BOTTOM: Convergence to tracer equilibrium states under boundary conditions $q_{M}=$ $1, q_{H}=0$ under standard (dashed) and preconditioned (solid) integration. . 138

4.5 Schematic illustrating grid box tracer equilibration as the expansion of the ventilated subvolume. As new waters enter the grid box, the ventilated fraction increases until it occupies the entire grid box at equilibrium. . . . . . . 139

4.6 Four-box tracer transport model schematic modified from Figure 2.9. Tracer concentration values $q_{M}$ and $q_{H}$ in two mixed-layer boxes are injected into box 1 at rates $F_{M}=1 \mathrm{~Sv}$ and $F_{H}=9 \mathrm{~Sv}$, and $q_{H}$ is injected into box 2 at rate $F_{H}=9 \mathrm{~Sv}$. Fluid is homogenized in box 1 and flows into box 2 at a rate $F_{M}+F_{H}=10 \mathrm{~Sv}$, from which it eventually leaves the system. . . . . . . 143

4.7 Evolution of tracer concentrations (solid lines) and extrapolated equilibrium values (dashed lines) in the intermediate (top) and deep (bottom) boxes of \begin{tabular}{|l|l|l|}
\hline the idealized model in Figure & 4.6 & The left column corresponds to to surface
\end{tabular} boundary conditions $q_{M}=1, q_{H}=0$ and the right column to $q_{M}=0, q_{H}=1$. 144

4.8 Passive tracer time step as a function of vertical level in the 15-layer model used for forward tracer simulations. . . . . . . . . . . . . . . . . 147

4.9 Constant (in time) surface concentration boundary conditions of preformed oxygen in $\mathrm{mol} \mathrm{O} / \mathrm{m}^{3}$. Values do not exceed 0.4 . 
4.10 Top: Maximum values of $\tilde{C}_{\infty}(t)$ over all depths and times. Locations where values are larger than roughly $0.4 \mathrm{~mol} \mathrm{O} \mathrm{m}^{-1}$ are affected by numerical artifacts. Bottom: Comparison at different locations of preformed oxygen concentrations, $C(t)$, and equilibrium estimates, $\tilde{C}_{\infty}(t)$, for vertically synchronous and asynchronous time steps computed over a 1250 year MITgcm simulation using $\varepsilon=0.05$. Grid box locations are given by black circles in the top panel. Large positive excursions in the Southern Ocean estimates arise from numerical artifacts. Extrapolated, vertically asynchronous estimates converge most quickly at all locations. Dotted vertical lines lie at 100 years, the duration integration used in Section $\mid 4.5\rceil$. . . . . . . . . . . 149

4.11 Same as Figure 4.10 but for ideal age and only computed using vertically synchronous time stepping. In the top panel the maximum values of $\tilde{C}(t)$ have been computed over all depths and times greater than 500 years due to the prevalence of overshoots during initial centuries of simulation. . . . . . 152

4.12 Passive tracer time step as a function of vertical level in the 50-layer model used for adjoint tracer simulations. . . . . . . . . . . . . 154

4.13 Top: Regions of nonzero tracer boundary conditions (blue), hypothetical data (red), and plotted sections (cyan lines) overlaid on model bathymetry used in the idealized adjoint experiment. Second: Fraction of AABW computed by Johnson [2008] along a Western Atlantic section similar to that shown in the top panel. Third: Western Atlantic section of extrapolated passive tracer concentrations after 100 years of integration before any control adjustments are made. Bottom: Western Atlantic section showing the vertical distribution of hypothetical data. . . . . . . . . . . . . . 158

4.14 Distributions of passive tracer concentration released south of $60^{\circ} \mathrm{S}$ after 100 years shown for the initial simulation (top row), after control adjustments computed in the first iteration (middle) and their difference (bottom). Left, center, and right columns correspond to sections in the Western Atlantic, Eastern Atlantic, and Pacific Oceans, respectively, as illustrated by the cyan lines in the top panel of Figure 4.13 . . . . . . . . . . . . . . . . 160

4.15 Top: Differences in tracer concentrations along the Western Atlantic section between the initial simulation and a forward simulation using only the control changes derived for $\kappa_{G M}$. Most of the shoaling of AABW seen when all controls are used is captured by changing only this variable. Middle: Changes in $\kappa_{G M}$ derived in the first iteration of the idealized tracer inverse experiment. Units are $\mathrm{m}^{2} \mathrm{~s}^{-1}$. Bottom: $C_{\infty, 0}^{A A B W}$ in the 100th year of simulation at $3900 \mathrm{~m}$. Most changes in $\kappa_{G M}$ lie in regions of large background

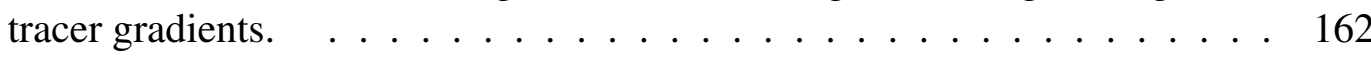


4.16 Left: Differences in tracer concentrations along the Western (top) and Eastern (bottom) Atlantic sections between the initial simulation and a forward simulation using only the wind stress control changes derived to reduce model misfit to hypothetical $C^{A A B W}$ in the sensitivity experiment with decreased $\sigma_{\kappa_{\sigma}}, \sigma_{\kappa_{G M}}$, and $\sigma_{\kappa_{d}}$. Right: Wind stress changes data in Pa, shown for June; other months are qualitatively similar. Bottom: resulting meridional velocity anomaly $\left(\mathrm{m} \mathrm{s}^{-1}\right)$ at $3700 \mathrm{~m}$. Positive values indicate a relative increase in northward velocity. . . . . . . . . . . . . . . . . . 164 


\section{List of Tables}

$2.1 \quad$ Summary of benthic foraminiferal $\delta^{18} \mathrm{O}_{c}$ records $\ldots \ldots . \ldots . \ldots 32$

2.2 "Code" indicates reference codes used in this paper for ease of discussion. SA1967 refers to a South Atlantic core at $1967 \mathrm{~m}$ depth, NI1580 to a North Indian core at $1580 \mathrm{~m}$ depth, etc. All species are from the genus Cibicidoides except for cores NA3146 (Planulina and Globobulimina) and EP3210 (Uvigerina). . . . . . . . . . . . . . . . . . 32

2.3 Summary of planktonic foraminiferal $\delta^{18} \mathrm{O}_{c}$ records . . . . . . . . . . . . . 34

2.4 "N." and "G." stand for Neogloboquadrina and Globigerina;"pachy" stands for pachyderma; "s." and "d." stand for sinistral and dextral. . . . . . . . . 34

2.5 Data importance for benthic records averaged over 20-10 ka calculated from the data resolution matrix $\mathbf{T}_{u} \ldots \ldots \ldots \ldots$. . . . . . . . 54

3.1 Control variables, prior control adjustment uncertainties $(\sigma)$, sources of first guesses, and period and duration for control elements used in the LGM state estimate. ECCO refers to the ECCO version 4 simulation in the year 2007, and averaged in space to be used on the lower-resolution llc45 grid. $\Delta$ CCSM4 refers to the LGM minus pre-industrial control runs in coupled CCSM4 simulations illustrated in Figure $3.4, \kappa_{\sigma}, \kappa_{d}$, and $\kappa_{G M}$ refer to coefficients of isopycnal diffusivity [Redi][1982], diapycnal diffusivity, and eddy bolus velocity [Gent and McWilliams, ]1990]. "Spin-up run" refers to the long forward simulation under ECCO $+\triangle \mathrm{CCSM} 4$ forcings described in Sec-

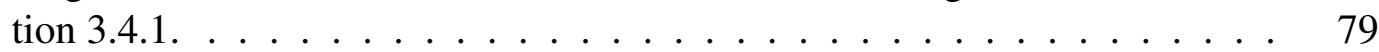

3.2 Contributions to the the cost function in the LGM state estimate. . . . . . . 84

4.1 Control variables, first guesses, prior control adjustment uncertainties $(\sigma)$, smoothing extents, and period and duration for control elements used in the idealized adjoint simulation. All values are taken from the ECCO v4 simulation in the year 2007, and averaged in space to be used on the lowerresolution grid. $\kappa_{\sigma}, \kappa_{d}$, and $\kappa_{G M}$ refer to coefficients of isopycnal diffusivity [Redi, 1982], diapycnal diffusivity, and eddy bolus velocity [Gent and McWilliams, 1990]. . . . . . . . . . . . . . 156

4.2 Contributions to the the cost function in the idealized adjoint simulation. . 156 


\section{Chapter 1}

\section{Introduction}

Observations suggest that during the last deglaciation (roughly 20,000-10,000 years ago) the Earth warmed substantially, global mean sea level rose roughly 130 meters in response to melting ice sheets and glaciers, and atmospheric concentrations of carbon dioxide increased. The ocean plays a key role in the modern climate system by storing and transporting heat, salt, carbon, and nutrients, but its role during deglacial climate change remains uncertain. Understanding how past ocean states differed from the modern, and how the ocean changes in changing climates, addresses questions about the stability and geometry of the modern ocean circulation. This thesis expands on previous efforts to reconstruct the ocean circulation over the last glacial interval and ensuing deglaciation by developing and applying new frameworks for combining ocean models and paleoceanographic data. The structure of the thesis is as follows:

Chapter 2. Inferring surface water equilibrium calcite $\delta^{18} \mathrm{O}$ during the last deglacial period from benthic foraminiferal records: Implications for ocean circulation

This chapter evaluates the ability of last-deglacial equilibrium calcite $\delta^{18} \mathrm{O}\left(\delta^{18} \mathrm{O}_{c}\right)$ 
records to constrain hydrographic changes hypothesized in the literature by Skinner and Shackleton [2005], Waelbroeck et al. [2006], and Waelbroeck et al. [2011], all of whom suggested that spatial variations in deglacial $\delta^{18} \mathrm{O}_{c}$ observed by benthic foraminifera could be the signature of past ocean circulation changes. In contrast to those results, Wunsch and Heimbach [2008] found that two cores with an apparently large phase lag were consistent with the modern ocean circulation, and Gebbie [2012] found deglacial histories of global ML seawater $\delta^{18} \mathrm{O}_{c}$ and temperature that reproduced the offset in a steady-state model of the modern tracer transport. In my Master of Science thesis [Amrhein, 2014], I developed techniques to infer transient and steady mixed layer (ML) tracer signals from deep ocean proxy observations using singular value decomposition.

Here I expand on these previous efforts by testing for circulation changes using both benthic ("downstream") and planktonic ("upstream") foraminifera, as well as quantifying the uncertainty of of ML tracer values inferred from benthic records and providing physical rationales for how uncertainties vary in space and time. A history of $\mathrm{ML} \delta^{18} \mathrm{O}_{c}$ spanning the last deglaciation is inferred from a least-squares fit of eight benthic foraminiferal $\delta^{18} \mathrm{O}_{c}$ records to Green's functions estimated for the modern ocean circulation. Disagreements between this history and the ML history implied by planktonic records would indicate deviations from the modern circulation. No deviations are diagnosed because the two estimates of ML $\delta^{18} \mathrm{O}_{c}$ agree within their uncertainties, but analyses of singular vectors suggest data collection and modeling procedures useful for inferring circulation changes in future studies. Uncertainties of benthic-derived ML $\delta^{18} \mathrm{O}_{c}$ are lowest in the high-latitude regions chiefly responsible for ventilating the deep ocean; additional high-resolution planktonic records constraining these regions are of particular utility. Benthic records from the Southern Ocean, where data are sparse, appear to have the most power to reduce uncertainties in benthicderived ML $\delta^{18} \mathrm{O}_{c}$. Understanding the spatiotemporal covariance of deglacial ML $\delta^{18} \mathrm{O}_{c}$ will also improve abilities of $\delta^{18} \mathrm{O}_{c}$ records to constrain deglacial circulation. 
Chapter 3: GOSE: A Glacial Ocean State Estimate

Among the best-documented features of the Last Glacial Maximum (LGM, roughly 20,000 years ago) is the cooling of near-surface temperatures in the atmosphere and ocean. Proxy evidence for cooler near sea-surface ocean temperatures compiled by the Multiproxy Approach for the Reconstruction of the Glacial Ocean Surface [MARGO, Waelbroeck et al., 2009] includes data from fossil shells of several species and multiple analytical techniques along with uncertainty estimates. Regional variations in ocean cooling in the proxies suggest changes in the roles of the atmosphere and ocean in transporting, transferring, and storing heat at the LGM. However, many of these variations are not reproduced in numerical models run under glacial boundary conditions as part of the Paleoclimate Modeling Intercomparison Project [PMIP; Otto-Bliesner et al., 2007, Braconnot et al., 2007, Tao et al., 2013, Dail and Wunsch, 2014]. The inability of models to reproduce apparently robust large-scale temperature changes at the LGM represents a gap in our understanding of how the climate was different during that time; it is unknown whether missing physics or other model errors are driving the misfit, or whether it arises from biases in the observations.

The ECCO (Estimating the Circulation and Climate of the Ocean) framework for ocean state estimation is a powerful tool combining a primitive equation ocean model (the MIT$\mathrm{gcm}$ ) with a Lagrange multiplier procedure for reduction of model-data misfit. Dail] [2012b] and Dail and Wunsch [2014] used this tool to derive a simulation of the LGM Atlantic Ocean constrained by MARGO. This work extends those efforts by using a global ocean domain, increasing model run durations, and including additional information about LGM climate by using atmospheric forcing fields generated by a PMIP model. Model forcings adjusted to fit the model to data include seasonal cycles of winds, precipitation, radiation, humidity, and surface air temperature, as well as time-invariant horizontal and vertical mixing parameter adjustments. The model has 50 vertical levels and active sea ice, permitting resolution of abyssal water mass distributions and sea ice buoyancy forcing. 
The inferred "current best estimate" of the ocean circulation is colder than the modern ocean, with more vigorous barotropic circulation than in the modern ocean, but the data do not appear to require a qualitatively different role for the LGM ocean in the climate system. While the simulated abyssal ocean circulation differs from the modern, it appears that the MARGO data have little ability to constrain quantities like the Atlantic Meridional Overturning Circulation strength (in agreement with Kurahashi-Nakamura et al. [2013]) or global meridional heat transport. An assessment of $\delta^{18} \mathrm{O}_{c}$ observations suggests that those data have the potential to to constrain deep ocean properties, but that they may be limited by sparsity and noise.

\section{Chapter 4: Ocean state estimation from passive tracer observations}

This chapter continues in the vein of Chapter 2 by seeking to infer circulation changes from observations of deep-ocean tracers. However, in contrast with Chapter 2 and with previous inverse modeling efforts that relied on kinematic or geostrophic models [LeGrand and Wunsch, 1995, Huybers et al., 2007, Gebbie and Huybers, 2006, Marchal and Curry, 2008, Burke et al., 2011, Lund et al., 2011, Dail, 2012a, Gebbie, 2014], this chapter demonstrates how to fit a primitive equation coupled ocean-ice model (the MITgcm) to observations of passive tracers. Using a primitive equation model is advantageous because it has superior handling of high-latitude convection, which is important for setting deep ocean circulation and tracer values [Gjermundsen and LaCasce, submitted], and because it allows us to investigate the influences of a wide range of model variables (atmospheric forcings and turbulent transport parameterizations) in fitting the data. Also, unlike Chapter 2 and previous studies that have found that changes in tracer boundary conditions can fit paleo data under the modern circulation, this chapter assumes fixed boundary conditions and infers circulation changes that fit the data. Finally, this chapter uses synthetic observations - constructed to 
represent the scenario where Antarctic Bottom Waters in the Atlantic shoaled, a popular hypothesis for glacial intervals - in an idealized experiment. This work paves the way for future idealized experiments, where hypothetical scenarios that may be supported by a range of circumstantial evidence that may be difficult to include explicitly in an inverse model can be schematized by synthetic data and possible physical scenarios derived using the adjoint machinery.

Much of the chapter is dedicated to developing two numerical techniques for fitting the MITgcm to observations of passive tracers using the method of Lagrange multipliers; previously, such approaches had been severely limited by attendant computational costs. First, scaling time steps within model grid boxes by the inverse of the local Courant number is shown to be equivalent to a Jacobian preconditioning of tracer transport that minimizes the convergence time to equilibrium, generalizing the approach of Bryan [1984]. Second, I describe an extrapolation procedure that can reduce the amount of integration time required to estimate equilibrium tracer distributions by a factor of 10 or more in some ocean locations. These techniques may be useful in related fields (e.g., chemical or biological oceanography) where tracer equilibration times are a substantial computational burden. 


\title{
Chapter 2
}

\section{Inferring surface water equilibrium}

\section{calcite $\delta^{18} \mathrm{O}$ during the last deglacial}

\author{
period from benthic foraminiferal
}

\section{records: Implications for ocean}

\section{circulation}

This chapter is an extension of my MS thesis [Amrhein, 2014], which developed methods for inferring mixed layer (ML) tracer concentrations from deep ocean measurements using a tracer transport model. Here I apply that machinery to the problem of inferring deglacial ocean circulation changes by comparing estimates of $\mathrm{ML} \delta^{18} \mathrm{O}$ of equilibrium calcium carbonate derived from benthic and planktonic foraminifera. The quantification of solution uncertainties is further developed in this chapter, and a column-scaling approach is described in Appendix 2.7.1 that yields ML tracer solutions that are smooth in space.

This chapter is the accepted version of the following article: Amrhein, D.E., G. Gebbie, 
O. Marchal, and C. Wunsch (2015), Inferring surface water equilibrium calcite $\delta^{18} \mathrm{O}$ during the last deglacial period from benthic foraminiferal records: Implications for ocean circulation, Paleoceanography, 30(11), 1470-1489, which has been published in its final form at https://dx.doi.org/10.1002/2014PA002743. Used with permission as granted in the original copyright agreement.

\subsection{Introduction}

Changes in the physical and chemical properties of the ocean were an important component of the global climate change of the last deglaciation, the period roughly 20-10 calendar kyr B.P. (ka), when Earth's atmosphere and ocean warmed and ice sheets retreated. Numerous studies have investigated possible large and (or) abrupt deviations from the modern circulation during this time using numerical models [Manabe and Stouffer, 1988, Otto-Bliesner et al., 2007, Liu et al., 2009, Barker et al., 2009], proxy observations [Keigwin, 2004, McManus et al., 2004, Curry and Oppo, 2005], and combinations of models and data [LeGrand and Wunsch, 1995, Winguth et al., 2000, Gebbie and Huybers, 2006, Burke et al., 2011, Marchal and Curry, 2008, Dail and Wunsch, 2014, Gebbie, 2014]. Distinguishing between possible circulation changes is challenging because the available database is sparse and has numerous sources of uncertainty.

The oxygen isotope ratio, $\delta^{18} \mathrm{O}$, of foraminiferal shells extracted from sediment cores has been used for decades as an indicator of global ice volume and climate change [e.g., Hays et al. 1976]. Foraminiferal measurements of $\delta^{18} \mathrm{O}$ are a proxy for the equilibrium calcite oxygen isotope ratio, $\delta^{18} \mathrm{O}_{c}$, which is a function of temperature and the ambient oxygen isotope ratio of seawater, $\delta^{18} \mathrm{O}_{w}$ [e.g., Bemis et al., 1998]. Increasing temperatures and melting land ice sheets both decrease $\delta^{18} \mathrm{O}_{c}$ in the surface ocean, so that changes in $\delta^{18} \mathrm{O}_{c}$ 
are indicative of major deglacial climate change processes.

Regional variations in benthic proxy observations of $\delta^{18} \mathrm{O}_{c}$ have been interpreted in terms of various physical processes and changes, including (1) changes in ocean circulation, (2) changes in mixed layer (ML) $\delta^{18} \mathrm{O}_{c}$ values, (3) delays due to ocean tracer propagation times, and (4) observational, representational, and age model error. Skinner and Shackleton [2005] suggested that an apparent 4000-year offset in the onset of deglaciation between $\delta^{18} \mathrm{O}_{c}$ in sediment cores TR163-31B (3210 m, eastern Equatorial Pacific) and MD99-2334K (3146 m, Iberian Margin) could be explained by circulation changes in the deep Atlantic Ocean. Waelbroeck et al. [2006] interpreted reductions at $16 \mathrm{kyr}$ B.P. (ka) in $\delta^{18} \mathrm{O}_{c}$ and the carbon isotope ratio $\delta^{13} \mathrm{C}$ at site MD98-2165 (2100 m, Indian Ocean) as the signature of a poorly-ventilated, brine-generated intermediate water mass that propagated to the Indian Ocean from the North Atlantic via a "fast connection" (simultaneous to within dating uncertainty). Waelbroeck et al. [2011] found signals of the last deglaciation in the Atlantic appearing earliest in waters at roughly $1000 \mathrm{~m}$ water depth and concluded that a delayed onset of deglaciation in waters deeper than $3000 \mathrm{~m}$ is consistent with reduced advection of $\delta^{18} \mathrm{O}_{c}$ signals in deep waters during Heinrich Stadial 1 (17.5-16.5 ka). In contrast, Wunsch and Heimbach [2008] estimated time scales of tracer equilibration and concluded that the 4000-year offset in cores MD99-2334K and TR163-31B is consistent with the modern ocean circulation, and Gebbie [2012] found deglacial histories of global ML seawater $\delta^{18} \mathrm{O}$ and temperature that reproduced the offset in a steady-state model of the modern tracer transport. It is not clear whether these interpretations are in disagreement because no uncertainty estimates were provided by the studies.

This paper evaluates the ability of last-deglacial $\delta^{18} \mathrm{O}_{c}$ records to constrain hydrographic changes hypothesized in the literature. Planktonic and benthic records lie "upstream" and "downstream" in the global ocean tracer transport in the sense that tracer values are first set in the mixed layer, near the habitats of planktonic foraminifera, and are subsequently 
transported to abyssal habitats of benthic foraminifera. Modifications in $\mathrm{ML} \delta^{18} \mathrm{O}_{c}$ signals as they are communicated to the deep ocean are set by properties of the ocean circulation; in principle, some of these properties can be estimated by comparing upstream and downstream observations of $\delta^{18} \mathrm{O}_{c}$. An analog is a one-dimensional advective-diffusive system ("pipe flow") with transiting tracer pulses, wherein the degree of smoothing of an upstream tracer signal en route to a downstream observer is related to the diffusivity, and delays in arrival times are indicative of flow speed. Relationships between arrival times, smoothing, and transport properties are much more complex in the turbulent, three-dimensional global ocean. Here, rather than attempting directly to infer flow speeds or diffusivities, we develop an upstream-downstream framework to address the more basic question of whether a compilation of deglacial benthic and planktonic records can reject the null hypothesis that the modern circulation persisted throughout the last deglaciation.

The procedure is to compare deglacial ML $\delta^{18} \mathrm{O}_{c}$ histories from (1) a least-squares fit of eight benthic $\delta^{18} \mathrm{O}_{c}$ benthic records to an estimate of the modern circulation, and (2) thirteen planktonic $\delta^{18} \mathrm{O}_{c}$ records. Disagreements (beyond uncertainty) between benthicand planktonic-derived ML $\delta^{18} \mathrm{O}_{c}$ estimates would disprove the null hypothesis and provide information about deglacial circulation changes.

In the present application, $\mathrm{ML} \delta^{18} \mathrm{O}_{c}$ estimates derived from planktonic and benthic foraminifera do not disagree given their uncertainties. This result does not mean that the circulation did not change over the deglaciation, merely that the data and assumptions lack the power to detect those changes. As additional data are gradually being generated and new age models constructed, this study should be regarded as providing a tentative set of conclusions about the power of foraminiferal $\delta^{18} \mathrm{O}_{c}$ to constrain past circulations and establishing a very general method for using additional data as they become available. The main contributions of this work are a means to quantify uncertainty in the problem of inferring past circulation from ocean tracer records, an understanding of physical processes contributing to 
that uncertainty, and the identification of steps to reduce uncertainty.

\subsection{Benthic and planktonic $\delta^{18} \mathbf{O}_{c}$ records}

Eight sediment core records of benthic $\delta^{18} \mathrm{O}_{c}$ (Table 2.1) were selected based on their resolution in time (to minimize errors due to aliasing) and the availability of age models that are not derived by synchronizing $\delta^{18} \mathrm{O}_{c}$ records to $\delta^{18} \mathrm{O}_{c}$ at other core sites, which can destroy information about the timing of tracer signals. These data are a representative, but not complete, subset of previously-studied records satisfying these criteria. Most have been used in previous studies on the propagation of $\delta^{18} \mathrm{O}_{c}$ signals in the ocean [Skinner and Shackleton, 2005, Waelbroeck et al., 2006, 2011]. The data coverage is far from ideally suited to the problem; discussion of an ideal set of core locations is postponed until Section 2.5. All $\delta^{18} \mathrm{O}_{c}$ values are reported and plotted in units of permil VPDB.

For easier reference, benthic records are labeled in this study by ocean basin and recovery depth rather than cruise number (e.g., SA3770 is the core recovered from the South Atlantic at $3770 \mathrm{~m}$ water depth; see Table 2.1). Five of the records were obtained from the Atlantic Ocean, two from the Indian Ocean, and one from the Pacific Ocean (Figure 2.1). Water depths of the sediment cores range from $1299 \mathrm{~m}$ to $3770 \mathrm{~m}$. All of the records but SA3770 come from continental margins, and all but two are based on tests from the genus Cibicidoides; $\delta^{18} \mathrm{O}_{c}$ records for cores NA3146 and EP3210 are derived at least in part from other genera.

Chronologies of benthic $\delta^{18} \mathrm{O}_{c}$ records were derived primarily from radiocarbon ages of planktonic foraminifera extracted from the same sediment core. For all records but two (SA1967 and NA1299) the published age models are used in this study. In SA1967 and NA1299 the published age models were constrained in part by tuning those records to ben- 


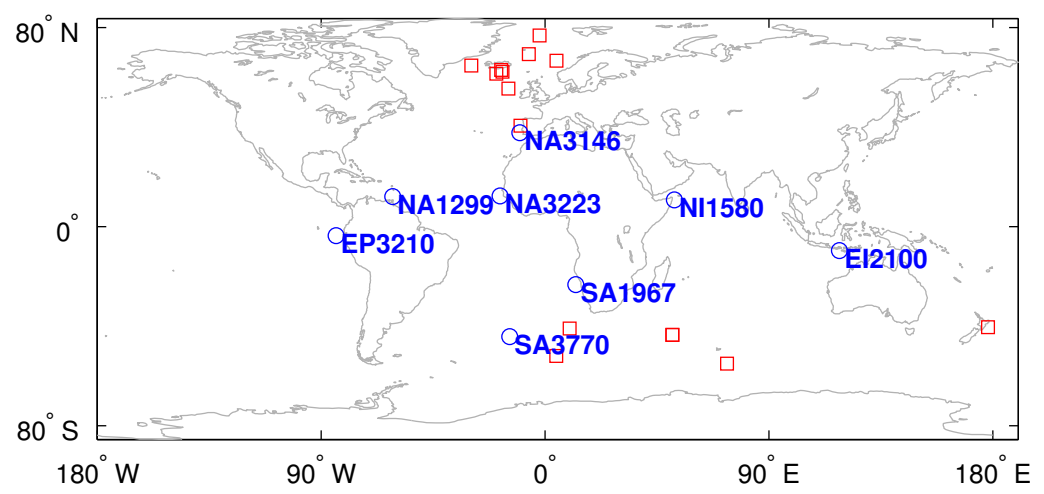

Figure 2.1: Locations of the eight benthic (blue, with labels) and 13 planktonic (red squares) records of $\delta^{18} \mathrm{O}_{c}$ used in this study. Locations and names of planktonic records are listed in Table 2.3 .

thic $\delta^{18} \mathrm{O}_{c}$ from other sediment cores; new age models for those cores were constructed by computing calendar ages from published ${ }^{14} \mathrm{C}$ ages using OxCal 4.1 [Bronk Ramsey, 2009. Reimer et al., 2009] and linearly interpolating between ${ }^{14} \mathrm{C}$-derived ages with depth. Previously published age models for NA3146 and NA3223 used in this study rely in part on synchronization of planktonic $\delta^{18} \mathrm{O}_{c}$ records derived from those cores to planktonic $\delta^{18} \mathrm{O}_{c}$ records for cores SU81-18 [Bard et al., 1987] and MD95-2042 [Shackleton et al., 2004], respectively.

Each benthic $\delta^{18} \mathrm{O}$ record shows a deglacial decrease of 1-2\%o over the interval from 25-5 ka, but the timing of these transitions appears asynchronous with the age models used (Figure 2.2). The $\delta^{18} \mathrm{O}_{c}$ decrease in core EP3210 appears markedly later than in other records. Records at shallower depths (NI1580, EI2100, SA1967, and NA1299) appear to share a twostage decrease in $\delta^{18} \mathrm{O}$, with the first transition occurring between roughly $18 \mathrm{ka}$ and 16 ka.

Only temporal anomalies of sediment core records relative to each record's time mean are considered in this paper. Subtraction of record time means mitigates effects of interlaboratory and interspecies $\delta^{18} \mathrm{O}_{c}$ offsets [e.g., Ostermann and Curry, 2000] as well as a small nonconservative effect due to pressure. Isolation of record anomalies is permitted by 


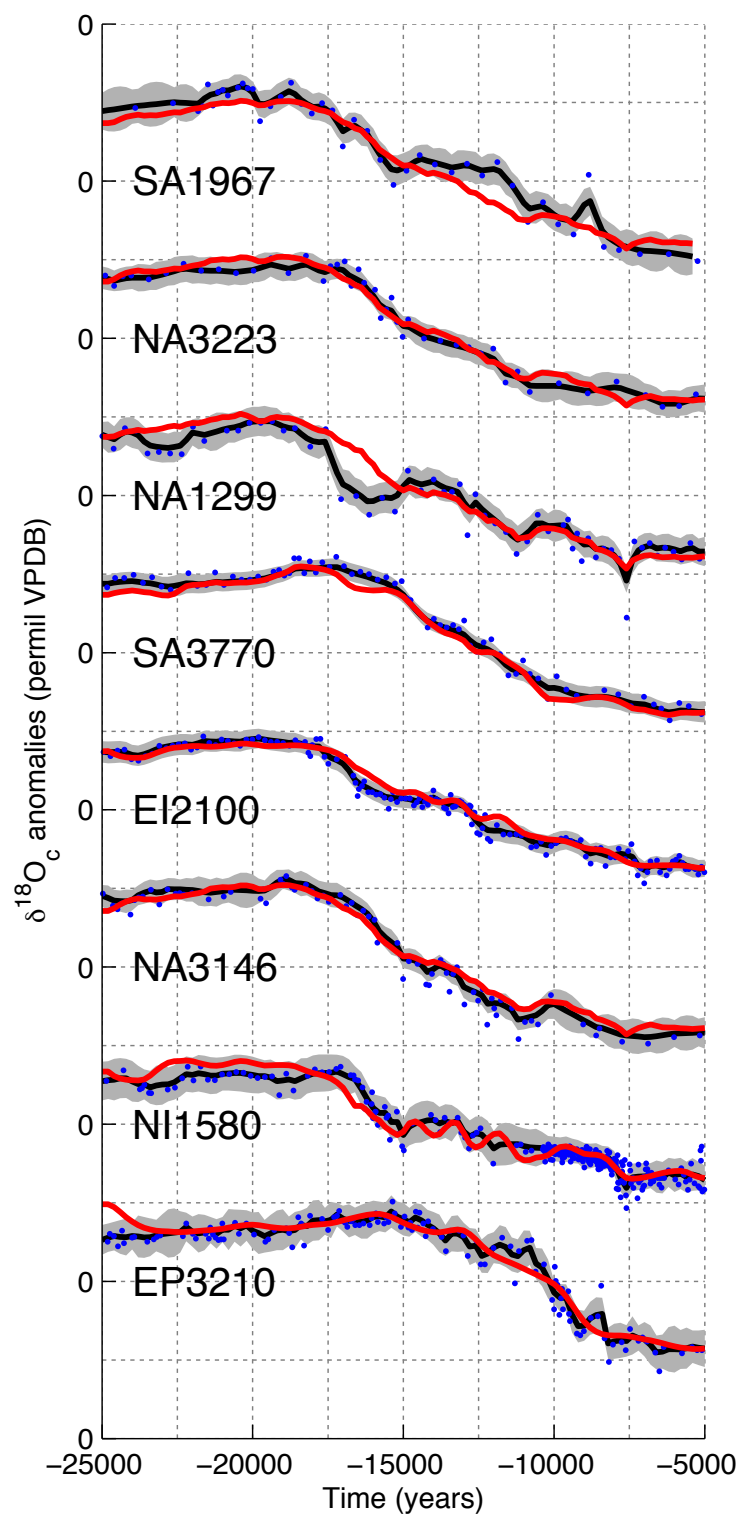

Figure 2.2: Eight benthic foraminiferal $\delta^{18} \mathrm{O}_{c}$ anomaly records are used to infer ML conditions. Blue dots show the published data, the solid black line their objectively mapped values, and the grey shading the objectively mapped uncertainty. Vertical grid spacing is $1 \%$ and record order is arbitrary. Red lines show the reconstructed data (see text) derived from the ML $\delta^{18} \mathrm{O}_{c}$ solution obtained in Section 2.4 with the small effect due to initial conditions added back. 


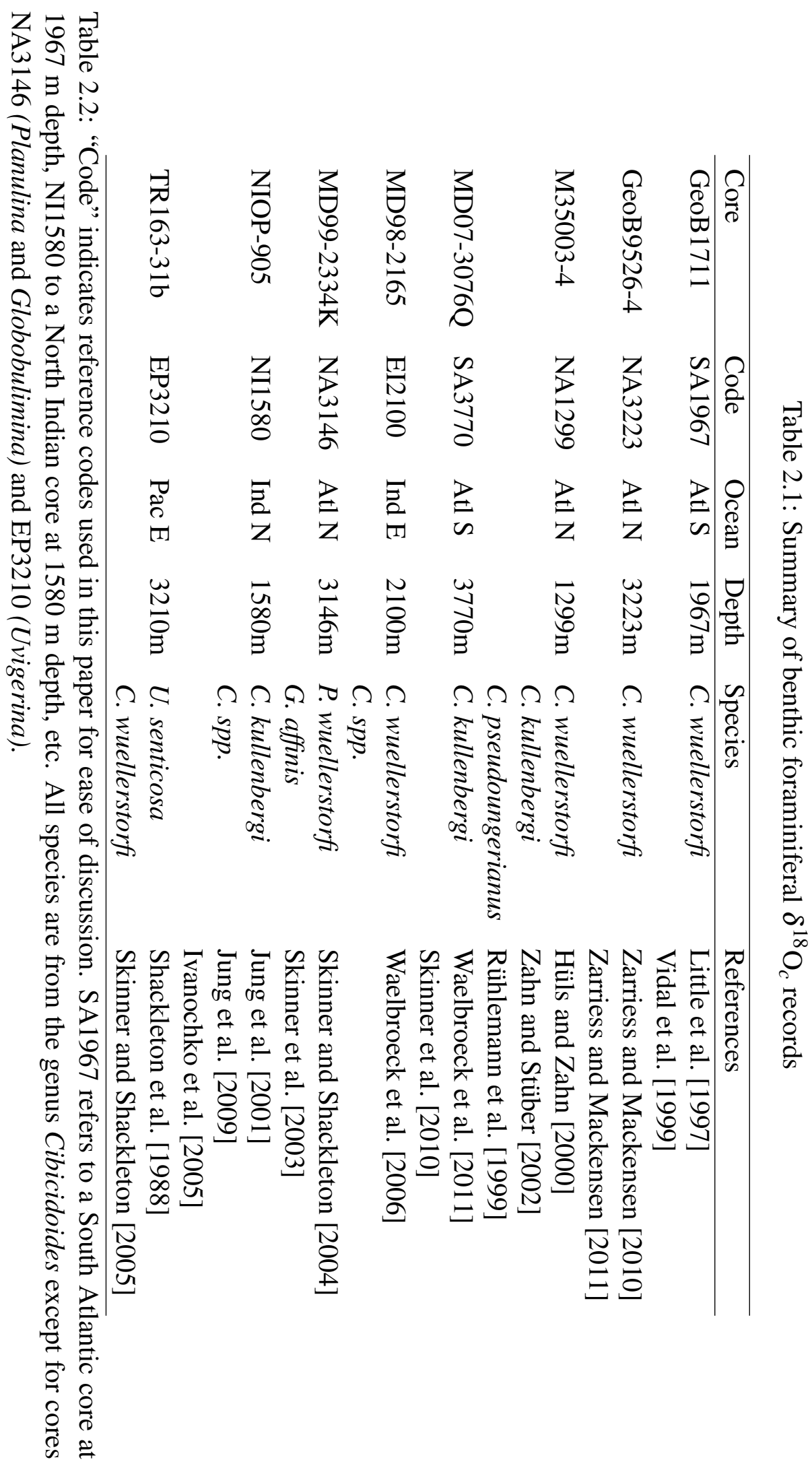


the linearity of passive tracer transport; in solving the inverse problem using the anomalies, it is assumed that record means, anomalies, and their respective uncertainties are uncorrelated. The separate inverse problem of finding a history of $\mathrm{ML} \delta^{18} \mathrm{O}_{c}$ satisfying the record time means is ignored because record means are biased from true means by finite record lengths.

Benthic $\delta^{18} \mathrm{O}_{c}$ anomaly records may be decomposed into "signal" and "noise" components, where the signal is the true in situ $\delta^{18} \mathrm{O}_{c}$ and the noise consists of supposedly random variations due to observational and representational errors. Signal and noise are both assumed to be Gaussian processes. Explicit noise statistics are required to use the data quantitatively; here, following LeGrand and Wunsch [1995], Gebbie and Huybers [2006], and Marchal and Curry [2008], observational errors are described by zero-mean white noise with standard deviation $\sigma_{n}=0.2 \%$.

Due to variable sampling procedures and sedimentation rates, measurements within each benthic $\delta^{18} \mathrm{O}_{c}$ record are spaced irregularly in time. To avoid preferential fits in the leastsquare procedure to benthic records during densely-sampled time periods, objective mapping [e.g., Bretherton et al., 1976, Wunsch, 2006] is used to generate time series that are regularly spaced at 200-year intervals spanning $25 \mathrm{ka}$ to $5 \mathrm{ka}$ (Figure 2.2). To compute objectively mapped values, a statistically stationary estimate of the signal autocovariance, $R(\tau)=\langle s(t) s(t+\tau)\rangle$, is necessary for each record, where $\tau$ is a time lag, $s$ is the $\delta^{18} \mathrm{O}_{c}$ record signal component, and angle brackets indicate the expected value. Signal autocovariances are calculated by fitting a power law to the structure function, $V(\tau) \equiv$ $\frac{1}{2}\left\langle[s(t+\tau)-s(t)]^{2}\right\rangle=\left\langle s^{2}(t)\right\rangle-R(\tau)$, computed between every two points in each record [Press et al., 1992, Rybicki and Press, 1992, Amrhein, 2014]; the signal variance, $\left\langle s^{2}(t)\right\rangle$, is approximated from the record variances and $\sigma_{n}$. The resulting interpolation (Figure 2.2) has uncertainties determined by $\sigma_{n}$, the estimate of $R(\tau)$, and the data distribution. Because objectively mapped values are linear combinations of observations, uncertainty is autocorrelated in time within each record. Uncertainty is assumed to be uncorrelated between records 
Table 2.3: Summary of planktonic foraminiferal $\delta^{18} \mathrm{O}_{c}$ records

\begin{tabular}{|c|c|c|c|c|}
\hline Core & Latitude & Longitude & Species & References \\
\hline PS1243-1 & $69.4^{\circ} \mathrm{N}$ & $6.6^{\circ} \mathrm{W}$ & N. pachy s. & Bauch et al. [2001] \\
\hline MD95-2010 & $66.7^{\circ} \mathrm{N}$ & $4.6^{\circ} \mathrm{E}$ & N. pachys. & Dokken and Jansen [1999] \\
\hline GIK23519-5 & $64.8^{\circ} \mathrm{N}$ & $29.6^{\circ} \mathrm{W}$ & N. pachys. & Millo et al. [2006] \\
\hline P69 & $40.4^{\circ} \mathrm{S}$ & $178^{\circ} \mathrm{E}$ & G. bulloides & Nelson et al. [2000] \\
\hline ODP177-1089 & $40.9^{\circ} \mathrm{S}$ & $9.9^{\circ} \mathrm{E}$ & G. bulloides & Hodell et al. [2003] \\
\hline MD73-025 & $43.5^{\circ} \mathrm{S}$ & $51.2^{\circ} \mathrm{E}$ & N. pachys. & Labracherie et al. [1989] \\
\hline MD84-527 & $43.5^{\circ} \mathrm{S}$ & $51.2^{\circ} \mathrm{E}$ & N. pachys. & Pichon et al. [1992] \\
\hline RC13-271 & $52.0^{\circ} \mathrm{S}$ & $4.5^{\circ} \mathrm{E}$ & N. pachy s./d. & Charles et al. [1991] \\
\hline MD84-551 & $55^{\circ} \mathrm{S}$ & $73.2^{\circ} \mathrm{E}$ & N. pachy s. & Pichon et al. [1992] \\
\hline MD95-2040 & $40.6^{\circ} \mathrm{N}$ & $10.1^{\circ} \mathrm{W}$ & G. bulloides & Schönfeld et al. [2003] \\
\hline ODP980 & $55.5^{\circ} \mathrm{N}$ & $14.7^{\circ} \mathrm{W}$ & N. pachy s. & $\begin{array}{l}\text { D. Oppo, J. McManus, } \\
\text { pers. comm. }\end{array}$ \\
\hline
\end{tabular}

Table 2.4: "N." and "G." stand for Neogloboquadrina and Globigerina;"pachy" stands for pachyderma; "s." and "d." stand for sinistral and dextral.

because observational and representational error processes at distant core sites are likely to be independent.

Thirteen planktonic $\delta^{18} \mathrm{O}_{c}$ records (Figure 2.6, Table 2.3) were selected on the basis of resolution, presence of ${ }^{14} \mathrm{C}$-derived age models, duration, and location in the high-latitude North Atlantic or Southern Oceans (Section 2.4). Seven records were derived from Neogloboquadrina pachyderma (sinistral), five from Globigerina bulloides, and one from N. pachyderma (both sinistral and dextral). Original published age models are used. All age models are derived from ${ }^{14} \mathrm{C}$ dates on planktonic foraminifera and/or by correlation to ice core records, with the caveat that several models may not include updated ${ }^{14} \mathrm{C}$ reservoir ages [e.g., Skinner et al., 2010]. Each record has a glacial-interglacial transition of 1-2\%. Spatial variations among planktonic $\delta^{18} \mathrm{O}_{c}$ records are due to local and regional variability as well as record noise. 


\subsection{Data analysis}

\subsubsection{Relating benthic proxies to ML conditions}

A tracer transport model is used to relate sediment core benthic $\delta^{18} \mathrm{O}_{c}$ records to mixed-layer $\delta^{18} \mathrm{O}_{c}$ histories. Similar to Lund et al. [2011] and Gebbie [2012], the procedure treats $\delta^{18} \mathrm{O}_{c}$ as conservative everywhere, except for source contributions in the ML. Experimental studies [e.g., Bemis et al. 1998] show that $\delta^{18} \mathrm{O}_{c}$ measured on foraminifera is well represented by a linear function of seawater $\delta^{18} \mathrm{O}, \delta^{18} \mathrm{O}_{w}$, and temperature, $T$. Non-conservative pressure effects on $T$ yield biases of $0.02 \%-0.07 \%$ for each core depending on depth and latitude [Fofonoff and Millard, 1983, Bryden, 1973, Saunders, 1981]. This effect is mitigated by the subtraction of record means and is otherwise neglected.

To illustrate the data analysis method generically, define first a state vector, $\mathbf{c}(t)=$ $\left[c_{1}(t), c_{2}(t), \ldots c_{N}(t)\right]^{\top}$, where $c_{i}(t)$ is $\delta^{18} \mathrm{O}_{c}$ at a time $t$ in the $i^{t h}$ of $N$ grid boxes subdividing the ocean domain below the ML. Likewise define the vector $\mathbf{q}(t)=\left[q_{1}(t), q_{2}(t), \ldots q_{L}(t)\right]^{\top}$ to be the $\delta^{18} \mathrm{O}_{c}$ of $L$ ML points. Evolution of the state vector is written in the canonical form

$$
\mathbf{c}(t+\Delta t)=\mathbf{A c}(t)+\mathbf{F q}(t) .
$$

Lower case and upper case bold variables are vectors and matrices, respectively. The matrix A is the so-called state transition matrix, and $\mathbf{F}$ describes the influence of $\mathbf{q}(t)$ on the state at the time $t+\Delta t$ [Wunsch, 2006]. Here we assume (from the null hypothesis) that $\mathbf{A}$ and $\mathbf{F}$ are steady and known from the modern circulation.

Define $\mathbf{y}(t)=\left[y_{1}(t), y_{2}(t), \ldots y_{M}(t)\right]^{\top}$ to be the objectively mapped benthic $\delta^{18} \mathrm{O}_{c}$ anomalies (Section 2.2 from $M$ sediment cores, and let $\mathbf{n}(t)=\left[n_{1}(t), n_{2}(t), \ldots n_{M}(t)\right]^{\top}$ be the

errors in those anomalies. Benthic $\delta^{18} \mathrm{O}_{c}$ anomalies are expressed as noisy measurements of 
the model state, $\mathbf{c}(t)$,

$$
\mathbf{y}(t)=\mathbf{B c}(t)+\mathbf{n}(t)
$$

where the matrix $\mathbf{B}$ relates benthic $\delta^{18} \mathrm{O}_{c}$ anomalies at sediment core locations to $\delta^{18} \mathrm{O}_{c}$ at the nearest grid boxes. Offsets of 200-300 m exist in several cases between model grid box centers and core depths, effects of which are not considered.

To write a single matrix equation relating the time history of $\mathrm{ML} \delta^{18} \mathrm{O}_{c}$ to benthic observations at all times, define concatenated vectors of values ordered first in space and then in time as underlined vectors,

$$
\begin{aligned}
& \underline{\mathbf{y}}=\left[\mathbf{y}\left(t_{0}\right)^{\top}, \mathbf{y}\left(t_{0}+1 \Delta t\right)^{\top}, \ldots, \mathbf{y}\left(t_{0}+S \Delta t\right)^{\top}\right]^{\top} \\
& \underline{\mathbf{n}}=\left[\mathbf{n}\left(t_{0}\right)^{\top}, \mathbf{n}\left(t_{0}+1 \Delta t\right)^{\top}, \ldots, \mathbf{n}\left(t_{0}+S \Delta t\right)^{\top}\right]^{\top} \\
& \underline{\mathbf{q}}=\left[\mathbf{q}\left(t_{0}\right)^{\top}, \mathbf{q}\left(t_{0}+1 \Delta t\right)^{\top}, \ldots, \mathbf{q}\left(t_{0}+S \Delta t\right)^{\top}\right]^{\top}
\end{aligned}
$$

where $S$ is the number of times when observations are present and $t_{0}$ is the initial such time. The initial conditions, data, noise, and ML conditions are related by

$$
\underline{\mathbf{y}}=\underline{\mathbf{y}}_{0}+\underline{\mathbf{G}} \underline{\mathbf{q}}+\underline{\mathbf{n}}
$$

where the matrix $\underline{\mathbf{G}}$ is described in Appendix 2.7.1 and $\underline{\mathbf{y}}_{0}$ is the contribution due to initial conditions, which nearly vanishes after 5000 years and is set to zero. As it is not the focus of this study, $\underline{\mathbf{y}}_{0}$ is estimated separately as $\underline{\mathbf{y}}_{0}$ (not shown) by forward propagating a least squares fit of time-constant ML $\delta^{18} \mathrm{O}_{c}$ to benthic anomalies averaged over -25000 - -20000 years. Defining $\underline{\mathbf{y}}^{*}=\underline{\mathbf{y}}-\underline{\mathbf{y}}_{0}$ and ignoring uncertainties in the estimation of $\underline{\mathbf{y}}_{0}$, the observations and 
ML conditions at all times are related by

$$
\underline{\mathbf{y}}^{*}=\underline{\mathbf{G}} \underline{\mathbf{q}}+\underline{\mathbf{n}} \text {. }
$$

This equation is solved to estimate the history of $\mathrm{ML} \delta^{18} \mathrm{O}_{c}(\underline{\mathbf{q}})$ from benthic $\delta^{18} \mathrm{O}_{c}$ anomalies $(\underline{\mathbf{y}})$ in the presence of a modern ocean circulation model ( $\underline{\mathbf{G}})$ and record noise ( $\underline{\mathbf{n}}$ ).

The matrix $\underline{\mathbf{G}}$ is constructed from adjoint Green's functions, which are computed by integrating a circulation model backwards in time for 5000 years under Heaviside conditions at each grid box representing a $\delta^{18} \mathrm{O}_{c}$ record (i.e., at each of those grid boxes, $c(t)=0$ for $t<0$ and $c(t)=1$ for $t \geq 0$, with $c(t)=0$ at all other grid boxes and times) and differencing the output in time. Tracer contributions after 5000 years are set to zero, which is accurate to within $10^{-5}$. The present approach is closely related to applications using Transit Time Distributions (TTDs), which are a form of Green's function [Peacock and Maltrud, 2006. Rutberg and Peacock, 2006].

The model used is a statistically steady-state representation of the combined effects of advection and mixing in the modern ocean circulation [Gebbie and Huybers, 2010, 2012, hereafter GH12]. Transports calculated in an ocean general circulation model (GCM) could also be used for this purpose; the GH12 model was chosen for its abilities to represent tracer distributions in the modern ocean and to approximate regions and rates of deep water formation, which are poorly simulated in most GCMs. The GH12 circulation was estimated using observations of potential temperature, salinity, phosphate, nitrate, and oxygen from a hydrographic compilation (WOCE and previous measurements, Gouretski and Koltermann [2004]), a gridded seawater $\delta^{18} \mathrm{O}$ product [LeGrande and Schmidt, 2006], and the GLODAP gridded dataset of bomb-corrected radiocarbon [Rubin and Key, 2002, Key et al., 2004]. The model domain has 33 vertical levels and a $4^{\circ} \times 4^{\circ}$ resolution with $L=2806 \mathrm{ML}$ regions whose vertical extent is defined using modern observed winter ML depth [Conkright et al., 
1994].

Figure 2.3 shows the adjoint Green's functions for cores EP3210 and NA3146. The method of empirical orthogonal functions [e.g., Preisendorfer and Mobley, 1988] was used to plot each adjoint Green's function as a single spatial pattern varying in time; in both cases this approximation captures more than $90 \%$ of the total spatiotemporal variance. The adjoint Green's functions show that tracer propagation to core sites smooths ML tracer anomalies in time and that outside of model high latitudes, tracer signals are minimally communicated to core sites. In the deep Pacific, the adjoint Green's function is broader in time than for the deep North Atlantic, qualitatively consistent with longer tracer equilibration time in basins far from deep water formation regions [Wunsch and Heimbach, 2008]. The NA3146 site is bathed primarily in deep water formed in the North Atlantic and Arctic, whereas the EP3210 site is mostly influenced by Southern Ocean waters.

The model time step used is $\Delta t=200$ years, the initial time is $t_{0}=-25000$ years, and the number of time steps is $S=101$, corresponding to reconstruction dates $t=-25000,-24800$, $\cdots-5000$ years. The data and noise, $\underline{\mathbf{y}}^{*}$ and $\underline{\mathbf{n}}$, are each $806 \times 1$ vectors whose length equals the total number of objectively-mapped observations, and $\underline{\mathbf{q}}$ is a $283406 \times 1$ vector whose length corresponds to the number of ML regions (2806) at every time (101) in the reconstruction. In total, 283406 unknown $\mathrm{ML} \delta^{18} \mathrm{O}_{c}$ values and 806 unknown observational errors are constrained by 806 objectively mapped benthic $\delta^{18} \mathrm{O}_{c}$ anomalies.

\subsubsection{Solution method for benthic-derived ML $\delta^{18} \mathbf{O}_{c}$}

The singular value decomposition [SVD; e.g., Wunsch, 2006] is used to find solutions for $\underline{\mathbf{q}}$ in Eq. 2.7. The SVD of $\underline{\mathbf{G}}$ is $\underline{\mathbf{G}}=\mathbf{U} \Lambda \mathbf{V}^{\top}$, where $\mathbf{U}$ and $\mathbf{V}$ are square orthonormal matrices and $\Lambda$ is a non-square diagonal matrix with $K$ strictly positive singular values $\lambda_{1}, \lambda_{2}, \ldots \lambda_{K}$ 


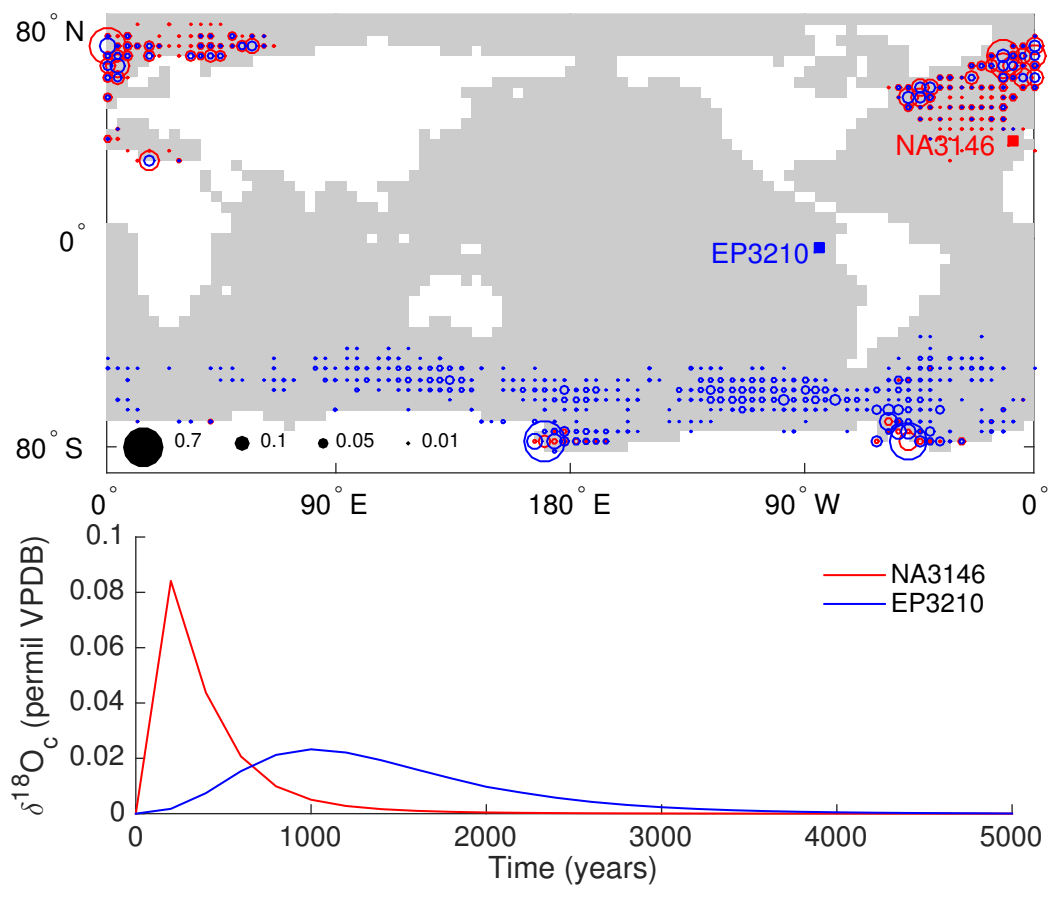

Figure 2.3: Adjoint Green's functions for NA3146 (red, Iberian Margin) and EP3210 (blue, Eastern Equatorial Pacific) computed using the GH12 model (see text). The adjoint Green's functions may be interpreted as follows. In the case when $\mathrm{ML} \delta^{18} \mathrm{O}_{c}$ conditions are a delta function in the $i^{t h}$ ML grid box at $t=0$ (that is, $q_{i}(t)=1 \%$ at $t=0$, and $q(t)=0 \%$ at all other places and times), the signal at each deep core site as the model is integrated forward in time is equal to the time trajectory in (b), scaled by the fraction indicated by circle area in the $i^{\text {th }}$ grid box in (a). Where there are no circles, tracer communicated to core sites is very small or zero. An approximation is made in representing the adjoint Green's functions by a pair of spatial and temporal patterns (see text); over $90 \%$ of the variability is accounted for at both core sites. 
in decreasing order along the diagonal. The $i^{t h}$ columns of $\mathbf{U}$ and $\mathbf{V}$ (the singular vectors) are written as $\mathbf{u}_{i}$ and $\mathbf{v}_{i}$, respectively. Denoting estimated values with a tilde, the general solution for $\underline{\mathbf{q}}$ computed by SVD is

$$
\underline{\tilde{\mathbf{q}}}=\sum_{i=1}^{K^{\prime}} \frac{\mathbf{u}_{i}^{\top} \underline{\mathbf{y}}^{*}}{\lambda_{i}} \mathbf{v}_{i}+\sum_{i=K^{\prime}+1}^{283406} \alpha_{i} \mathbf{v}_{i}
$$

In the solution, the first $K^{\prime}$ of the $\mathbf{v}_{i}$ (the effective range vectors) are weighted by the projection of the data, $\underline{\mathbf{y}}^{*}$, onto the corresponding $\mathbf{u}_{i}$ and inversely weighted by $\lambda_{i}$. The remaining $\mathbf{v}_{i}$ (the effective nullspace vectors) are weighted by unknown coefficients $\alpha_{i}$, all of which are set to zero, yielding the so-called truncated SVD solution. The effective rank, $K^{\prime} \leq K$, specifies the number of singular vectors retained in the solution (the effective range vectors) and is chosen based on the singular values, whose magnitudes are very low when data sparsity and/or noise preclude resolving the corresponding vectors.

Quantification of solution uncertainties is essential to compare benthic-derived ML $\delta^{18} \mathrm{O}_{c}$ to planktonic records. The truncated SVD solution uncertainty matrix, $\mathbf{P}$, describes the expected squared deviation of estimated ML $\delta^{18} \mathrm{O}_{c}$ from the true values [Wunsch, 2006],

$$
\begin{aligned}
\mathbf{P} & =\left\langle(\underline{\tilde{\mathbf{q}}}-\underline{\mathbf{q}})(\underline{\tilde{\mathbf{q}}}-\underline{\mathbf{q}})^{\top}\right\rangle \\
& =\sum_{i=1}^{K^{\prime}} \sum_{j=1}^{K^{\prime}} \mathbf{v}_{i} \frac{\mathbf{u}_{i}^{\top}\left\langle\underline{\mathbf{n}} \underline{\mathbf{n}}^{\top}\right\rangle \mathbf{u}_{j}}{\lambda_{i} \lambda_{j}} \mathbf{v}_{j}^{\top}+\sum_{i=K^{\prime}+1}^{283406} \sum_{j=K^{\prime}+1}^{283406} \mathbf{v}_{i}\left\langle\alpha_{i} \alpha_{j}\right\rangle \mathbf{v}_{j}^{\top} .
\end{aligned}
$$

Define the two components of $\mathbf{P}$ to be $\mathbf{P}_{c}$ and $\mathbf{P}_{\text {null }}$, respectively. The solution covariance, $\mathbf{P}_{c}$, describes uncertainty due to the observational error covariance, $\left\langle\underline{\mathbf{n n}}^{\top}\right\rangle$, (estimated by objective mapping in Section 2.2); the square root of its diagonal elements is the solution standard error. The contribution to the solution standard error from including the $i^{\text {th }}$ singular vector is proportional to $1 / \lambda_{i}^{2}$, indicating that $\mathbf{P}_{c}$ is highly sensitive to small values of $\lambda_{i}$. The nullspace uncertainty, $\mathbf{P}_{\text {null }}$, describes uncertainty arising from excluding the nullspace 
vectors. A tradeoff exists in choosing $K^{\prime}$ : as $K^{\prime} \rightarrow K, \mathbf{P}_{\text {null }}$ grows smaller as the number of effective nullspace vectors decreases, but $\mathbf{P}_{c}$ grows larger as small $\lambda_{i}$ are included in $\underline{\tilde{\mathbf{q}}}$. Here, $K^{\prime}$ is chosen based on the $\lambda_{i}$ with the goal of minimizing the total solution uncertainty.

The solution covariance, $\mathbf{P}_{c}$, is readily computed from the SVD and $\left\langle\underline{\mathbf{n}}^{\top}\right\rangle$, but additional a priori information about the nullspace weights, $\alpha_{i}$, is required for an estimate of $\mathbf{P}_{\text {null }}$. However, a relative measure of $\mathbf{P}_{\text {null }}$ is given by the solution resolution matrix, $\mathbf{T}_{v}$,

$$
\mathbf{T}_{v}=\mathbf{V}_{K^{\prime}} \mathbf{V}_{K^{\prime}}^{\top}
$$

where $\mathbf{V}_{K^{\prime}}=\left[\mathbf{v}_{1}, \ldots \mathbf{v}_{K^{\prime}}\right]$ is the semi-orthogonal matrix of right singular vectors in the effective range of $\underline{\mathbf{G}}$. The solution resolution matrix relates inferred and true $\mathrm{ML} \delta^{18} \mathrm{O}_{c}$,

$$
\underline{\tilde{\mathbf{q}}}=\mathbf{T}_{v} \underline{\mathbf{q}},
$$

in the absence of observational uncertainty and illustrates which features of $\underline{\mathbf{q}}$ lie in the nullspace and cannot be reproduced in $\underline{\tilde{\mathbf{q}}}$ [Wunsch, 2006]. Unresolvable features in time are due to low-pass filtering by ocean tracer propagation [Rutberg and Peacock, 2006, Amrhein, 2014], and unresolvable features in space are due to the sparsity of deep water formation regions and mixing in the ocean interior (Section 2.4. Appendix 2.7.3.

The pointwise resolvability is derived from $\mathbf{T}_{v}$ and describes the spatial distribution of $\mathbf{P}_{\text {null }}$, which is important for comparing $\underline{\tilde{\mathbf{q}}}$ to planktonic records. Consider the hypothetical scenario where the true $\mathrm{ML} \delta^{18} \mathrm{O}_{c}$ deglacial history, $\underline{\mathbf{q}}_{h y p, i}$, is $q_{0}$ in the $i^{\text {th }} \mathrm{ML}$ grid box and $0 \%$ everywhere else, at all times, over the last deglaciation. By Eq. 2.12, the reconstructed ML $\delta^{18} \mathrm{O}_{c}$ is $\tilde{\mathbf{q}}_{h y p, i}=\mathbf{T}_{v} \mathbf{q}_{h y p, i}$. Let $\tilde{q}_{0}$ be the deglacial time mean value of $\tilde{\mathbf{q}}_{h y p, i}$ in the $i^{\text {th }}$ 
grid box. The pointwise resolvability is defined as

$$
\begin{array}{r}
r_{i}=\frac{\tilde{q}_{0}}{q_{0}} \\
0 \leq r_{i} \leq 1
\end{array}
$$

and indicates what fraction of a ML $\delta^{18} \mathrm{O}_{c}$ signal in the $i^{\text {th }}$ grid box on the longest time scale will be reconstructed at the same location by the particular SVD solution. Insofar as ocean tracer transport acts as a low-pass filter, $r_{i}$ serves as an upper limit on the resolvability of shorter-timescale variability.

An additional diagnostic afforded by the SVD solution is the data resolution matrix,

$$
\mathbf{T}_{u}=\mathbf{U}_{K^{\prime}} \mathbf{U}_{K^{\prime}}^{\top}
$$

where $\mathbf{U}_{K^{\prime}}=\left[\mathbf{u}_{1}, \ldots \mathbf{u}_{K^{\prime}}\right]$ is the semi-orthogonal matrix of left singular vectors in the effective range of $\mathbf{G}$. The diagonal elements of $\mathbf{T}_{u}$ are the "data importance" and describe the relative importance of each observation in determining the solution [Wunsch, 2006].

Before computing the SVD, the problem is weighted to reflect observational uncertainty (derived from objective mapping, Section (2.2) ) and normalized by the column norms of $\mathbf{G}$ [Wunsch, 2006] to cancel apparent biases from spatial variations in vertical transport out of the ML (Appendix 2.7.3). Of the 283406 singular values of $\underline{\mathbf{G},} K=806$ are nonzero, corresponding to the number of objectively mapped observations at all times. Section 2.4 discusses results using $K^{\prime}=230$, chosen using a signal-to-noise criterion so that singular values less than the typical standard deviation of the objectively mapped observational noise lie in the nullspace. Larger values of $K^{\prime}$ improve the fit to the data (up to an exact fit for $K^{\prime}=806$ ), at the expense of rapidly increasing solution amplitudes and standard errors. Smaller values of $K^{\prime}$ yield inferior fits to the observations. 


\subsubsection{Relating planktonic proxies to ML conditions}

Planktonic $\delta^{18} \mathrm{O}_{c}$ observations (Section 2.2, written for all locations and times as the underlined vector $\underline{\mathbf{y}}^{p}$ ) and $\underline{\mathbf{q}}$ can be related in a generic linear form as

$$
\underline{\mathbf{y}}^{p}=\underline{\mathbf{R}} \underline{\mathbf{q}}+\underline{\mathbf{n}}^{p}
$$

where $\underline{\mathbf{R}}$ is a matrix describing which ML regions and times are constrained by planktonic observations (analogous to $\underline{\mathbf{G}}$ for benthic records) and $\underline{\mathbf{n}}^{p}$ is the planktonic observational noise. Planktonic-derived ML $\delta^{18} \mathrm{O}_{c}$ estimates are written $\underline{\tilde{\mathbf{q}}}^{p}$. Ideally, the matrix $\underline{\mathbf{R}}$ should represent plankton vital effects as well as covariances between $\delta^{18} \mathrm{O}_{c}$ in the ML and planktonic habitats, but these processes are poorly understood. Here, the simplifying assumption is made that planktonic records measure $\delta^{18} \mathrm{O}_{c}$ in the ML overlying their sediment core site; uncertainties in this representation are not assessed.

\subsection{Results}

\subsubsection{ML $\delta^{18} \mathbf{O}_{c}$ inferred from benthic data}

The truncated SVD solution, $\underline{\tilde{\mathbf{q}}}$, to Eq. 2.7 is a deglacial time history of $\mathrm{ML} \delta^{18} \mathrm{O}_{c}$ for all ML locations at $t=-25000,-24800, \cdots-5000$ years derived from eight benthic $\delta^{18} \mathrm{O}_{c}$ records. Nullspace uncertainties place strong limits on reconstruction accuracy, as diagnosed by the pointwise resolvability (Figure 2.4), which is non-uniformly distributed and generally small ( $r<0.01)$ outside of high latitudes. Low $r$ values imply that (1) only a small fraction of the true variability in each ML region is reconstructed and that (2) many other candidate 
solutions will fit the benthic data equally well. Evidently, in the context of the modern circulation, the benthic records effectively carry no information about the evolution of deglacial $\mathrm{ML} \delta^{18} \mathrm{O}_{c}$ outside of high latitudes. Understanding how high-latitude $\mathrm{ML} \delta^{18} \mathrm{O}_{c}$ covaried with values in other regions might permit benthic records to constrain those regions as well (Section 2.5).

The spatial distribution of pointwise resolvability is similar to that of the adjoint Green's functions (Figure 2.3), which in turn reflect dominant "ocean-filling" sites in the GH12 model (Gebbie and Huybers [2011], Figure 2). Clearly, only $\delta^{18} \mathrm{O}_{c}$ in ML regions that play a large role in ventilating the deep ocean can be reconstructed with any fidelity; the rationale is that only those regions have contributions to $\delta^{18} \mathrm{O}_{c}$ at core sites that are not dwarfed by observational noise. Appendix 2.7.3 demonstrates this tendency in a four-box model. As different modeled circulations have different surface regions that are most important for filling the deep ocean, the pointwise resolvability is sensitive to the steady circulation that is assumed.

Figure 2.4 plots $\underline{\tilde{\mathbf{q}}}$ as time series in five regions with nonzero $r$. All regions show glacialinterglacial transitions of $1 \% 0-2 \%$ superimposed upon several qualitatively different patterns of millennial and multi-millennial variability. Regions not plotted also have $1 \% 0-2 \% 0$ deglacial transitions, with similar patterns of variability. Examination of $\mathbf{P}_{c}$ (not shown) reveals that coherences within and between regions are highly uncertain and are likely due to data sparsity, as there is insufficient information to "unmix" contributions of different ML grid boxes to benthic core sites and to resolve spatial gradients in $\mathrm{ML} \delta^{18} \mathrm{O}_{c}$, so that spatially broad patterns are inferred (demonstrated in a four-box model in Appendix 2.7.3). The standard error (not shown) in $\mathrm{ML} \delta^{18} \mathrm{O}_{c}$ is between $0 \%$ and $0.6 \%$ at each time and location; in general, grid boxes with small pointwise resolvabilities have small standard errors because uncertainties in the data project minimally onto regions that are poorly constrained by the data. Together, the solution and pointwise resolvability suggest that the benthic data 
are able (weakly) to resolve weighted spatial averages of $M L \delta^{18} \mathrm{O}_{c}$ in regions of deep water formation, as well as grid-scale variability at locations whose contributions to ventilating the deep ocean in the model of GH12 are exceptionally high.

The reconstructed data, $\underline{\mathbf{y}}^{*}=\underline{\mathbf{G}} \underline{\tilde{\mathbf{q}}}$, is the $\delta^{18} \mathrm{O}_{c}$ reconstructed at benthic core locations when ML conditions are $\underline{\tilde{\mathbf{q}}}$ in an integration of the GH12 model over the deglaciation. Many characteristics of the data are reconstructed in $\underline{\mathbf{y}}^{*}$ (Figure 2.2): a deglacial transition of plausible amplitude is evident at every core site, no biases are apparent, and many record features are recovered. The reconstruction of a last-deglacial $\mathrm{ML} \delta^{18} \mathrm{O}_{c}$ history assuming the modern circulation that largely agrees with eight benthic records is not a trivial result. If such a history could not be found, then one might conclude that the misfit was due to a deviation from the modern circulation. The misfit to NA1299 during 18-16 ka can be reduced by choosing $K^{\prime}=500$, at the expense of introducing high-amplitude, millennial-scale variability and large standard errors to the solution in the Southern Hemisphere; even larger values of $K^{\prime}$ are necessary to reduce the misfit to SA1967 during 15-13 ka.

Spatially averaged time series of $\underline{\tilde{\mathbf{q}}}$ computed for the North Atlantic $\left(\tilde{m}_{N A}(t)\right)$ and the Southern Ocean $\left(\tilde{m}_{S O}(t)\right)$ are shown in Figure 2.5 (corresponding regions are shown in Figure 2.4 Appendix 2.7.2 describes the mean estimation procedure). Nullspace uncertainties are computed using two examples of possible covariance matrices $\left\langle\underline{\mathbf{q q}}^{\top}\right\rangle$; both assume that $\underline{\mathbf{q}}$ is Gaussian with zero mean and standard deviation equal to $1 \%$, with no autocorrelation in time. In the first example, no spatial covariance is imposed between ML grid boxes, and in the second example, ML $\delta^{18} \mathrm{O}_{c}$ is assumed hemispherically covarying and made to be spatially uniform within the Northern and Southern Hemispheres. Both cases likely underestimate the true nullspace uncertainty.

Both $\tilde{m}_{N A}(t)$ and $\tilde{m}_{S O}(t)$ have maximum values of $\delta^{18} \mathrm{O}_{c}$ between 21 and $19 \mathrm{ka}$ and minimum values at $8 \mathrm{ka}$, and solution variances are dominated by a roughly $2 \% 0$ glacialinterglacial transition. On the interval from $17 \mathrm{ka}$ to $11 \mathrm{ka} \tilde{m}_{N A}(t)$ is persistently less than 


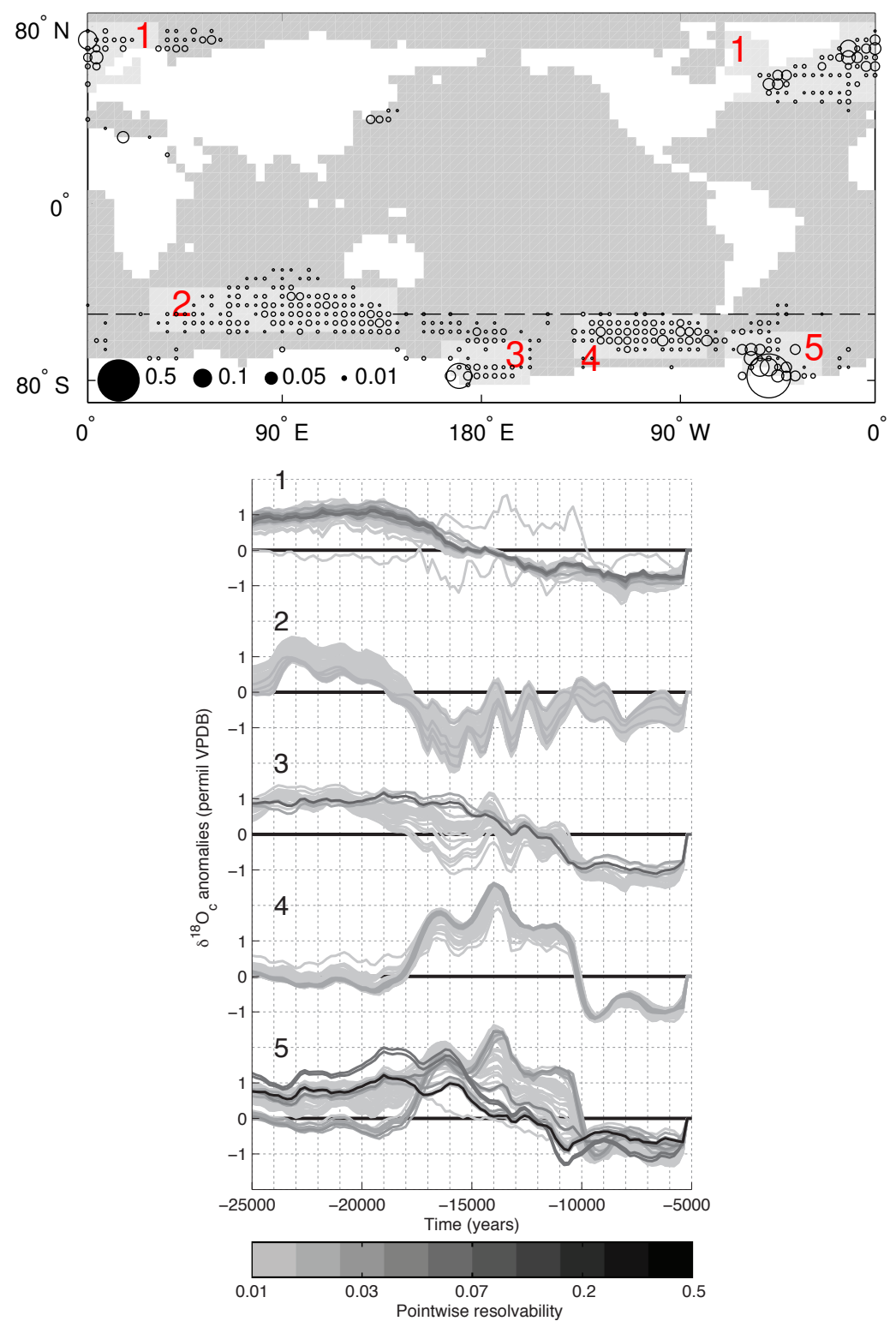

Figure 2.4: (a) Circle area centered on a grid box indicates the pointwise resolvability $r$; filled circles over land in the lower left are a legend. Grid points with no circles have resolvability $<0.01$. Lighter gray areas define regions plotted in the lower panel. Box 1 and the region south of the dotted line at $50^{\circ} \mathrm{S}$ are averaged to compute the North Atlantic and Southern Ocean time series $\tilde{m}_{N A}$ and $\tilde{m}_{S O}$, respectively (see text). (b) The benthic-derived ML $\delta^{18} \mathrm{O}_{c}$ solution plotted as overlaid time series at every grid box within the five geographical regions and shaded by pointwise resolvability. In all regions, values after $6 \mathrm{ka}$ lie in the nullspace and are equal to zero, as they are not constrained by benthic observations, which end at $5 \mathrm{ka}$. Note the nonlinear color bar. 


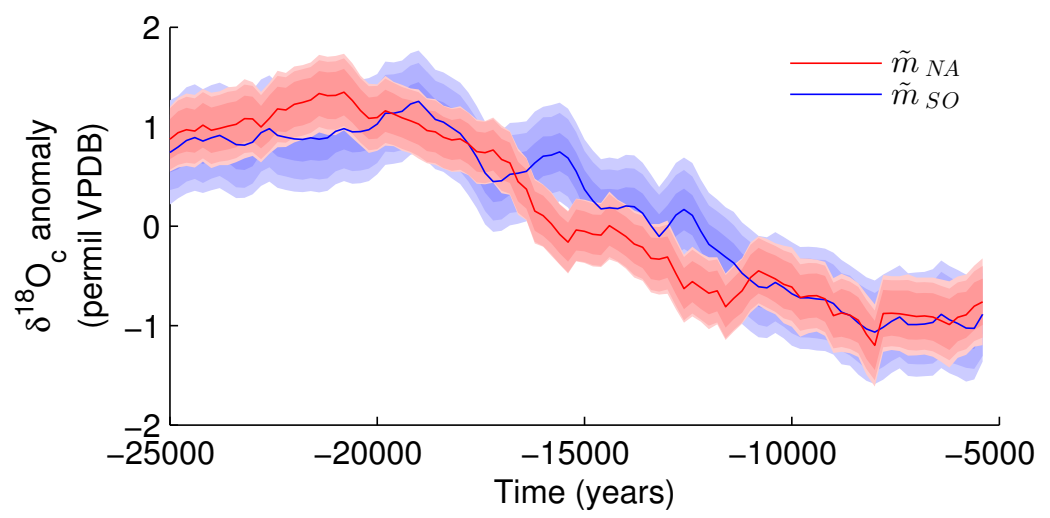

Figure 2.5: Estimates of ML $\delta^{18} \mathrm{O}_{c}$ anomalies in the North Atlantic $\left(\tilde{m}_{N A}\right)$ and Southern Oceans $\left(\tilde{m}_{S O}\right)$. The inner error bar is the standard error, the middle envelope includes the nullspace contribution when $\mathrm{ML} \delta^{18} \mathrm{O}_{c}$ is assumed hemispherically covarying, and the outer envelope includes the nullspace contribution when $\mathrm{ML} \delta^{18} \mathrm{O}_{c}$ is assumed spatially uncorrelated (see text).

$\tilde{m}_{S O}(t)$, with the largest differences $(0.8 \%$ and $0.7 \%)$ centered on $15.6 \mathrm{ka}$ and $12.6 \mathrm{ka}$. Uncertainty contributions due to $\mathbf{P}_{c}$ and $\mathbf{P}_{\text {null }}$ are comparable in size, and nullspace uncertainties are smaller when $\underline{\mathbf{q}}$ is assumed spatially uniform.

It is tempting to speculate about causes for interhemispheric variability in the inferred ML $\delta^{18} \mathrm{O}_{c}$, but given the large uncertainties, differences between $\tilde{m}_{N A}(t)$ and $\tilde{m}_{S O}(t)$ are not significantly different from zero. This result does not imply that spatially uniform ML $\delta^{18} \mathrm{O}_{c}$ in these two regions suffices to reconstruct the data, as was ruled out for NA3146 and EP3210 by Gebbie [2012], because the derived solution has substantial variation within and between the North Atlantic and Southern Ocean boxes (Figure 2.4).

Because ML $\delta^{18} \mathrm{O}_{c}$ histories are poorly constrained by benthic records, additional prior constraints on the solution could change its character substantially and reduce biases from the true solution. For instance, consideration of temperature and $\delta^{18} \mathrm{O}_{w}$ suggests that inferred $\delta^{18} \mathrm{O}_{c}$ values (Figure 2.4) in the Southern Ocean (SO) are biased high at the Last Glacial Maximum (LGM, approximately $23 \mathrm{ka}$ - $19 \mathrm{ka}$ ). Given the inferred LGM-Holocene anomaly of $\Delta \delta^{18} \mathrm{O}_{c}=2.25 \%$, using a paleotemperature equation [Bemis et al. 1998], $\delta^{18} O_{c}=$ 
$3.4+\delta^{18} O_{w}-0.21 T$, and attributing $1 \%$ to global ice volume differences [from pore water measurements, Adkins et al. 2002], LGM average SO ML temperatures are inferred to be $6^{\circ} \mathrm{C}$ less than in the Holocene, lower than the freezing point in much of the modern SO ML. (The effect of roughly $1 \mathrm{~g} / \mathrm{kg}$ higher global average salinities at the LGM on the freezing point of water is a depression of roughly $0.06^{\circ} \mathrm{C}$ [McDougall and Barker, 2011], too small to compensate for the inferred deviation.) For several reasons $\left(\Delta \delta^{18} \mathrm{O}_{c}\right.$ has a large uncertainty; local patterns of warming and cooling within the SO are possible; porewater-derived glacial $\delta^{18} \mathrm{O}_{w}$ may not be representative of the SO ML), we do not reject the solution, but the suggestion is that the solution may be "too cold" at the LGM and, more generally, that the derived solution may have persistent regional biases from true values. Treating temperature and $\delta^{18} \mathrm{O}_{w}$ as separate tracers and assigning an a priori zero probability to water temperatures below the freezing point could reduce the apparent bias; this direction is not explored further.

\subsubsection{Comparison of ML $\delta^{18} \mathrm{O}_{c}$ estimates from benthic and planktonic data}

The motivation for deriving $\underline{\tilde{\mathbf{q}}}$ was to compare it to ML conditions, $\underline{\mathbf{q}}^{p}$, estimated from planktonic foraminiferal $\delta^{18} \mathrm{O}_{c}$ records; misfits (beyond uncertainty) between the two estimates would indicate deviations from the modern circulation. Foraminiferal records considered were recovered from high latitudes, where pointwise resolvabilities are highest. Correlations between planktonic $\delta^{18} \mathrm{O}_{c}$ records and time series from $\underline{\tilde{\mathbf{q}}}$ in grid boxes nearest those records range between 0 and 0.96 in the Northern Hemisphere and between -0.32 and 0.63 in the Southern Hemisphere (Figure 2.6). Prolonged offsets between the two reconstructions are evident at most locations, and most features on millennial and shorter time scales 
in planktonic records are not visible in the benthic-derived reconstruction. However, low pointwise resolvabilities indicate that these disagreements are highly uncertain and do not suffice to disprove the null hypothesis that the modern circulation persisted throughout the deglaciation.

Regional averages $\tilde{m}_{N A}(t)$ and $\tilde{m}_{S O}(t)$ of $\underline{\tilde{\mathbf{q}}}$ are more robust and may have more power to test the null hypothesis. For comparison, Northern and Southern Hemispheric averages of planktonic records $\left(\tilde{m}_{N H}^{p}(t)\right.$ and $\tilde{m}_{S H}^{p}(t)$, respectively) are computed (Figure 2.7). Prior to computing averages, planktonic $\delta^{18} \mathrm{O}_{c}$ records were linearly interpolated to annual resolution, smoothed with a 200-year running mean, and subsampled at 200-year intervals to avoid overweighting means by more densely-sampled records. One-sigma uncertainties are approximated by standard deviations of planktonic records computed at every time step; given the sparsity of the data, these estimates should be regarded as lower limits.

Figure 2.8 compares $\tilde{m}_{N H}^{p}(t), \tilde{m}_{S H}^{p}(t), \tilde{m}_{N A}(t)$, and $\tilde{m}_{S O}(t)$. Ideally, a statistical test would be used to evaluate whether planktonic- and benthic-derived estimates agree within error. At present, with few planktonic records, a poor understanding of how and where they represent $\mathrm{ML} \delta^{18} \mathrm{O}_{c}$ (described by $\underline{\mathbf{R}}$, see Section 2.3.3), and lacking a robust quantification of uncertainty, we conclude that regional averages do not differ significantly and thus that the null hypothesis cannot be rejected even on larger spatial scales.

\subsection{Discussion}

It is worth considering whether the modern circulation null hypothesis is in any danger of being rejected in a statistically rigorous way by the available data. Several studies [LeGrand and Wunsch, 1995, Gebbie and Huybers, 2006, Marchal and Curry, 2008, Burke et al., 2011, Dail and Wunsch, 2014] have found that modern ocean circulation estimates adequately 

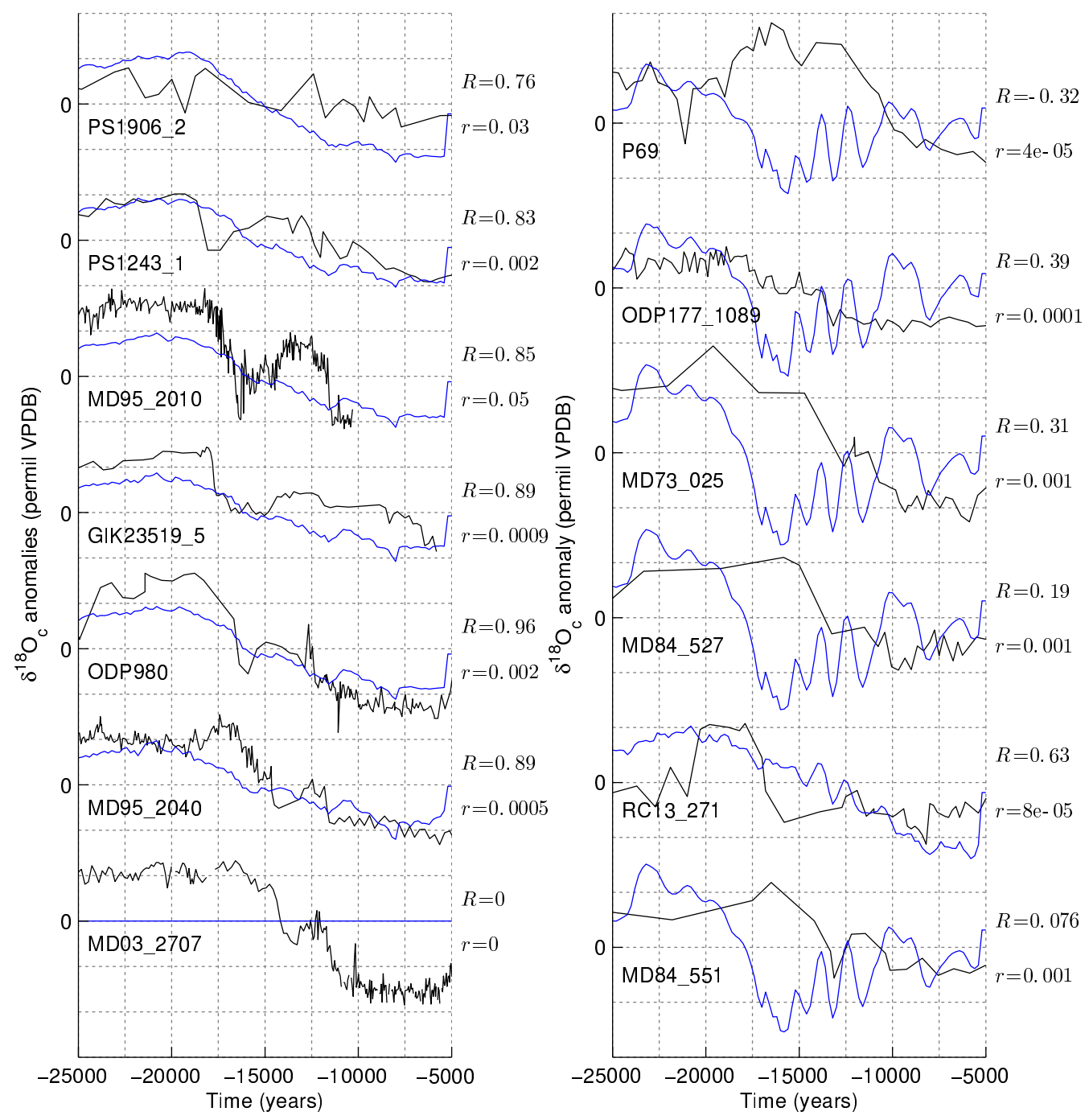

Figure 2.6: Comparison of thirteen planktonic $\delta^{18} \mathrm{O}_{c}$ anomaly records (black curves) and $\delta^{18} \mathrm{O}_{c}$ anomaly records derived from benthic records (blue curves) in the ML regions overlying planktonic core sites in the Northern (a) and Southern (b) Hemispheres. Panels are ordered by latitude. Pointwise resolvabilities, $r$ (Section 2.3), and Pearson correlations, $R$, are shown to the right of each panel. Very small values of $r$ (order $10^{-5}$ ) are practically indistinguishable from zero. The vertical grid spacing is $1 \%$. ML $\delta^{18} \mathrm{O}_{c}$ in the region overlying MD03_2707 lies entirely in the null space of the inverse problem $(r=0)$ and as such is inferred to be 0 at all times. 

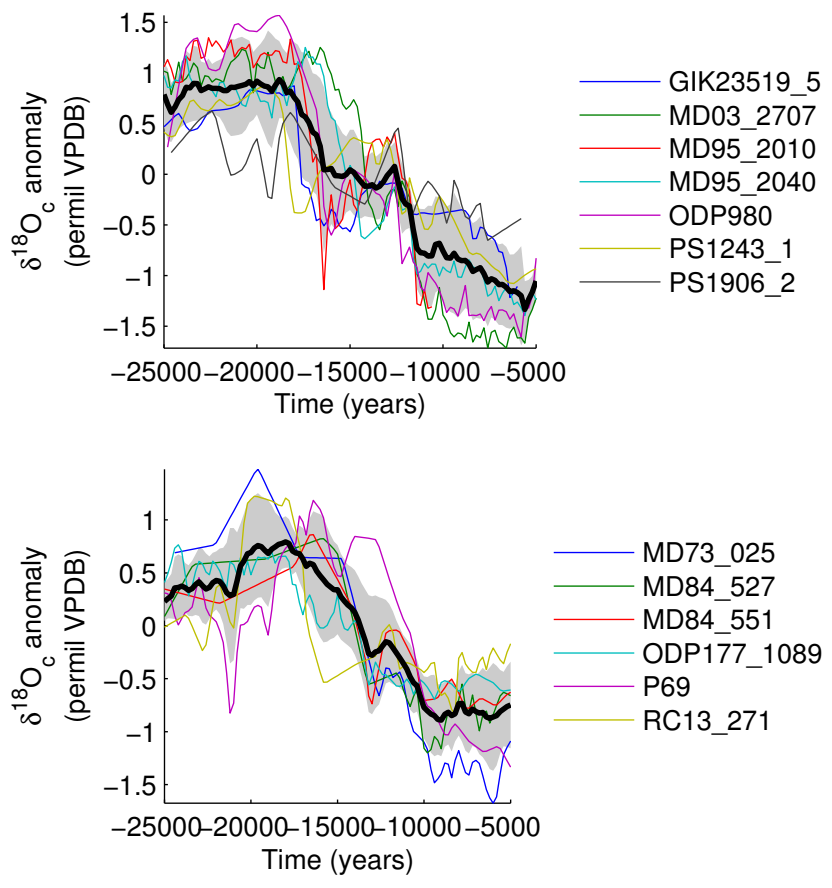

Figure 2.7: Northern (a) and Southern (b) Hemisphere planktonic $\delta^{18} \mathrm{O}_{c}$ anomalies overlaid with means removed. Thick black lines are the hemispheric time means computed after smoothing and subsampling each record (see text). Grey bars give the standard deviations of the records about the mean at each time. 

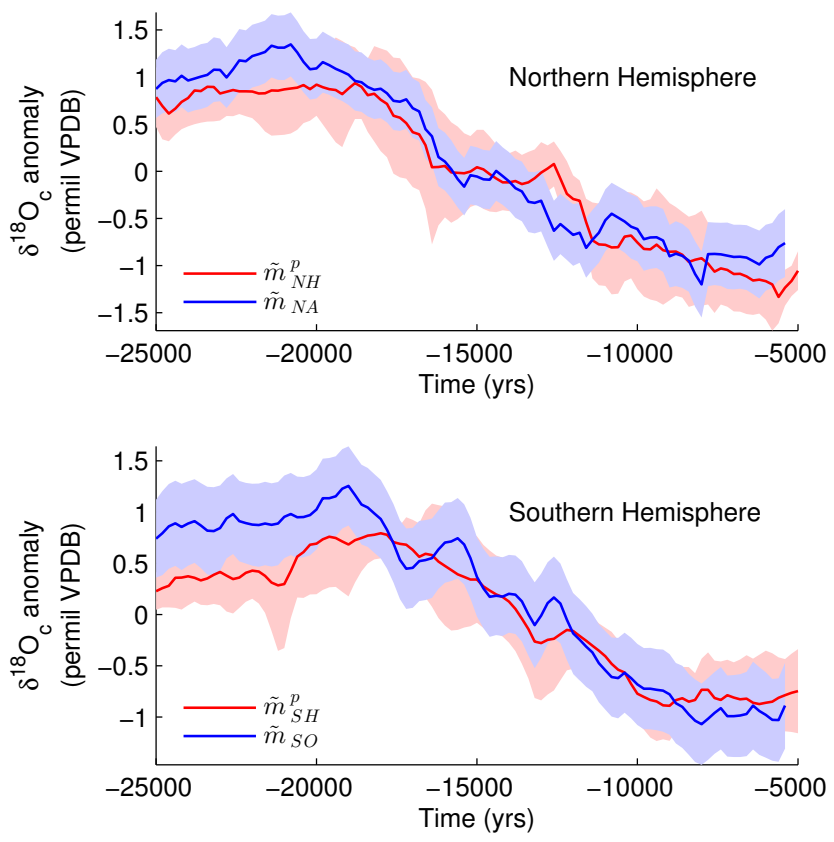

Figure 2.8: Comparison of spatially averaged $\mathrm{ML} \delta^{18} \mathrm{O}_{c}$ anomalies inferred from benthic records in the North Atlantic $\left(\tilde{m}_{N A}\right)$ and Southern $\left(\tilde{m}_{S O}\right)$ Oceans to averages of planktonic $\delta^{18} \mathrm{O}_{c}$ anomalies from the Northern and Southern Hemispheres $\left(\tilde{m}_{N H}^{p}\right.$ and $\tilde{m}_{S H}^{p}$, respectively). Uncertainties are the same as those described for Figures 2.5 and 2.7 . 
represent many observations from the LGM, though Gebbie [2014] suggested that $\delta^{13} \mathrm{C}$ measurements might disprove the null hypothesis at the LGM given additional constraints on surface distributions of that tracer. The LGM is arguably better constrained than the deglaciation because data are assumed to represent a steady state.

The modern circulation poses a difficult null hypothesis to reject using passive tracer observations because mixing, advection, and deep water formation processes filter tracer signals in space and time as they propagate downstream of the ML. Other candidate circulations (such as an ocean with a flushing time of $10^{6}$ years) could likely be readily rejected by the paleo data but would be less relevant to the central question of how past ocean states might have differed from the present. The challenge lies in generating a network of paleoceanographic observations with the power to test the null hypothesis and in representing those observations, along with salient a priori information, in an inverse model. The present framework for using upstream planktonic and downstream benthic records suggests ways forward on both fronts.

To test circulation hypotheses in the upstream-downstream framework, networks of benthic and planktonic records ideally should each be able to constrain all degrees of freedom (that is, all possible spatiotemporal structures) in $\mathrm{ML} \delta^{18} \mathrm{O}_{c}$. Short of that ideal, benthic and planktonic networks must be able to constrain a common subset of the spatiotemporal structures. In theory, two records (one benthic, one planktonic) could suffice to disprove the null hypothesis, provided their ML $\delta^{18} \mathrm{O}_{c}$ estimates had sufficiently low uncertainty; in practice, using multiple records of each type is more robust. Consideration of the nullspace of the benthic inverse problem suggests that low-frequency variability at high latitudes is the best target for reconstruction. Thus, additional high-resolution planktonic data representing high-latitude regions would be valuable, with the goal of estimating the $\mathrm{ML} \delta^{18} \mathrm{O}_{c}$ histories of waters that ultimately bathe benthic core sites. Similarly, benthic observations constraining $\mathrm{ML} \delta^{18} \mathrm{O}_{c}$ in regions where planktonic records are available would be useful. Benthic 
Table 2.5: Data importance for benthic records averaged over 20-10 ka calculated from the data resolution matrix $\mathbf{T}_{u}$

\begin{tabular}{cccccccc}
\hline SA1967 & NA3223 & NA1299 & SA3770 & EI2100 & NA3146 & NI1580 & EP3210 \\
\hline 0.16 & 0.30 & 0.29 & 0.94 & 0.12 & 0.25 & 0.17 & 0.13 \\
\hline
\end{tabular}

records recovered near deep water formation regions are expected to have better preservation of high-frequency ML signals.

The data importance (defined in Section ) indicates the relative roles of benthic sediment core records for constraining the present $\mathrm{ML} \delta^{18} \mathrm{O}_{c}$ solution. A value near 1 (the maximum) indicates that the solution is constrained to fit the corresponding observation well, whereas values near 0 (the minimum) indicate that the data minimally influence the solution and may be poorly reconstructed as a consequence. Data importance values for the eight benthic sediment core records averaged in time from 20 to $10 \mathrm{ka}$ (the shortened time interval avoids edge effects) range from 0.12 (EI2100) to 0.94 (SA3770) (Table 2.5). Cores in the North and South Atlantic near deep-water formation sites (where high-frequency ML signals have been attenuated less by ocean transport) have generally higher importance than records from the Indian or Pacific Oceans. The proximity of SA3770 to the Southern Ocean, which is important for setting $\delta^{18} \mathrm{O}_{c}$ in the global ocean and is poorly constrained by other records, presumably contributes to its particularly high importance.

Data importance can be computed for any hypothetical core location (given assumptions about the observational noise, which affects $\mathbf{T}_{u}$ ) and could be useful for choosing future core recovery sites. The broader question of sediment core location as it applies to experimental design in paleoceanography is a complex subject that should be addressed in a future study. Restrictions due to calcium carbonate preservation, sedimentation rates, bioturbation, and age model construction dramatically limit the set of available core sites.

Prior knowledge of how ocean ML $\delta^{18} \mathrm{O}_{c}$ varies in space and time (i.e. of $\left\langle\underline{\mathbf{q q}}{ }^{\top}\right\rangle$ ) would reduce the number of unconstrained degrees of freedom in the evolution of ML $\delta^{18} \mathrm{O}_{c}$, 
thereby making the problem of constraining its evolution more feasible. This information could be used by imposing smoothness constraints on the solution, as was done by Gebbie [2012] using modern distributions of temperature and $\delta^{18} \mathrm{O}_{w}$. In that study, solution values at high latitudes likely reflected constraints from observations, whereas values elsewhere reflected a priori constraints. Estimating $\left\langle\underline{\mathbf{q q}}^{\top}\right\rangle$ over the last deglaciation is challenging, as it is influenced by complex processes including surface ocean transport, patterns of evaporation and precipitation [Eisenman et al., 2009], and the spreading of low- $\delta^{18} \mathrm{O}_{c}$ waters from melting ice sheets [Condron and Winsor, 2011, 2012].

We have ignored uncertainties in record chronologies and assumed a best-case scenario in which published chronologies are correct. Inferences of past circulations from passive tracers are likely sensitive to chronological errors, which artificially phase-shift signals. Because the nullspace uncertainties are so large, it is unlikely that the fundamental results of this study would be changed by different core chronologies. Explicit descriptions of chronological uncertainties are critical for including that information in inverse modeling studies.

\subsection{Conclusions}

The inference of ML $\delta^{18} \mathrm{O}_{c}$ conditions is an important goal in paleoceanography, as surface $\delta^{18} \mathrm{O}_{c}$ changes are related to the Earth's climate. This work was performed with a further goal in mind - the eventual inference of past ocean circulation changes from passive tracers estimated upstream at the ocean surface and downstream in the abyss. Here, the modern ocean circulation was found to adequately represent planktonic and benthic $\delta^{18} \mathrm{O}_{c}$ records during the last deglaciation given the large uncertainties estimated for both types of record. This result does not disprove scenarios suggested by Waelbroeck et al. [2006], Waelbroeck et al. [2011], or Skinner and Shackleton [2005], but states that deviations from the modern 
circulation could not be diagnosed on the basis of the $\delta^{18} \mathrm{O}_{c}$ records considered under the assumptions used in this paper.

An improved understanding of how planktonic observations record $\mathrm{ML} \delta^{18} \mathrm{O}_{c}$ (especially at deep water formation sites, which are best constrained by benthic records) and how ML $\delta^{18} \mathrm{O}_{c}$ covaries in space and time is necessary if benthic and planktonic records are to be used in tandem with tracer transport models to constrain the circulation. Additional highlyresolved benthic $\delta^{18} \mathrm{O}_{c}$ records with reliable age models, especially those near deep water formation sites or in the Southern Ocean, will further constrain the solution towards the goal of rejecting the null hypothesis. The analyses presented are also well-suited to other geochemical tracers.

\subsection{Appendices}

\subsubsection{Statement of the inverse problem}

Applying Eq. (2.1) recursively, the tracer state vector at time $\mathbf{c}\left(t_{0}+s \Delta t\right)$ for any time step number $s=1,2, \ldots S$ is a linear function of the initial conditions and of the time history of $\mathbf{q}$,

$$
\mathbf{c}\left(t_{0}+s \Delta t\right)=\mathbf{A}^{s} \mathbf{c}\left(t_{0}\right)+\sum_{k=1}^{s} \mathbf{A}^{k-1} \mathbf{F} \mathbf{q}\left(t_{0}+(s-k) \Delta t\right) .
$$

Substituting Eq. 2.17) into Eq. 2.2 yields an expression relating the initial conditions, noise, and ML conditions to the observation $\mathbf{y}\left(t_{0}+s \Delta t\right)$,

$$
\mathbf{y}\left(t_{0}+s \Delta t\right)=\mathbf{B} \mathbf{A}^{s} \mathbf{c}\left(t_{0}\right)+\mathbf{B} \sum_{k=1}^{s} \mathbf{A}^{k-1} \mathbf{F} \mathbf{q}\left(t_{0}+(s-k) \Delta t\right)+\mathbf{n}\left(t_{0}+s \Delta t\right)
$$


Using $\underline{\mathbf{q}}($ Eq. (2.5)), Eq. (2.18) can be written

$$
\mathbf{y}\left(t_{0}+s \Delta t\right)=\mathbf{B A}^{s} \mathbf{c}\left(t_{0}\right)+\mathbf{B G}(s) \underline{\mathbf{q}}+\mathbf{n}\left(t_{0}+s \Delta t\right),
$$

where $\mathbf{G}(s)$ is the matrix (composed of model Green's functions) whose operation on $\underline{\mathbf{q}}$ gives the summation in Eq. (2.18). Together, the equations for $\mathbf{y}\left(t_{0}+s \Delta t\right)$ at all times $t_{0}, t_{0}+\Delta t, \ldots t_{0}+S \Delta t$ constitute a simultaneous set of linear equations in terms of $\mathbf{c}\left(t_{0}\right), \underline{\mathbf{q}}$, and $\mathbf{n}$,

$$
\left[\begin{array}{c}
\mathbf{y}\left(t_{0}\right) \\
\mathbf{y}\left(t_{0}+\Delta t\right) \\
\vdots \\
\mathbf{y}\left(t_{0}+S \Delta t\right)
\end{array}\right]=\left[\begin{array}{c}
\mathbf{B A}^{0} \\
\mathbf{B} \mathbf{A}^{1} \\
\vdots \\
\mathbf{B A}^{S}
\end{array}\right] \mathbf{c}\left(t_{0}\right)+\left[\begin{array}{c}
\mathbf{0} \\
\mathbf{B G}(1) \\
\vdots \\
\mathbf{B G}(S)
\end{array}\right] \underline{\mathbf{q}}+\left[\begin{array}{c}
\mathbf{n}\left(t_{0}\right) \\
\mathbf{n}\left(t_{0}+\Delta t\right) \\
\vdots \\
\mathbf{n}\left(t_{0}+S \Delta t\right)
\end{array}\right]
$$

Defining the matrices

$$
\underline{\mathbf{A}}=\left[\begin{array}{c}
\mathbf{B} \mathbf{A}^{0} \\
\mathbf{B} \mathbf{A}^{1} \\
\vdots \\
\mathbf{B A}^{S}
\end{array}\right], \underline{\mathbf{G}}=\left[\begin{array}{c}
\mathbf{0} \\
\mathbf{B G}(1) \\
\vdots \\
\mathbf{B G}(S)
\end{array}\right]
$$

and substituting $\underline{\mathbf{y}}$ and $\underline{\mathbf{n}}$ from Eq. (2.5), Eq. 2.20) becomes, collectively for all space and time,

$$
\underline{\mathbf{y}}=\underline{\mathbf{A}} \mathbf{c}\left(t_{0}\right)+\underline{\mathbf{G}} \underline{\mathbf{q}}+\underline{\mathbf{n}} \text {. }
$$


Defining $\underline{\mathbf{y}}_{0}=\underline{\mathbf{A}} \mathbf{c}\left(t_{0}\right)$ yields Eq. (2.6).

\subsubsection{Computation of regional averages and uncertainties}

Regional averages $\tilde{m}_{N A}(t)$ and $\tilde{m}_{S O}(t)$ are computed via least squares [Wunsch, 2006] as area- and uncertainty-weighted means of $\underline{\tilde{\mathbf{q}}}$ assuming that the deviations of $\underline{\tilde{\mathbf{q}}}$ about true regional means are described by $\mathbf{P}_{c}$; effects of this assumption are not assessed. Regional means are linear combinations of the elements in $\underline{\tilde{\mathbf{q}}}$, so it is possible to write (for the North Atlantic region; the same procedure is also used in the Southern Ocean) $\tilde{\mathbf{m}}_{N A}=\mathbf{M}_{N A}^{\prime} \underline{\tilde{\mathbf{q}}}$, where $\mathbf{M}_{N A}^{\prime}$ is the matrix specifying the mean estimation procedure and $\tilde{\mathbf{m}}_{N}$ is $\tilde{m}_{N A}(t)$ vectorized in

time, $\tilde{\mathbf{m}}_{N A}=\left[\tilde{m}_{N A}(0), \tilde{m}_{N A}(\Delta t), \ldots \tilde{m}_{N A}(S \Delta t)\right]^{\top}$. Similarly, define the matrix $\mathbf{M}_{N A}$ relating the true area-weighted regional average vector to the true ML conditions, $\mathbf{m}_{N A}=\mathbf{M}_{N A} \underline{\mathbf{q}}$. Using the definition of $\mathbf{T}_{v}$ (Eq. 2.12), the nullspace uncertainty due to approximating $\mathbf{m}_{N A}$ by $\tilde{\mathbf{m}}_{N A}$ is

$$
\begin{aligned}
& \left\langle\left(\mathbf{m}_{N A}-\tilde{\mathbf{m}}_{N A}\right)\left(\mathbf{m}_{N A}-\tilde{\mathbf{m}}_{N A}\right)^{\top}\right\rangle=\left\langle\left(\mathbf{M}_{N A} \underline{\mathbf{q}}-\mathbf{M}_{N A}^{\prime} \underline{\tilde{\mathbf{q}}}\right)\left(\mathbf{M}_{N A} \underline{\mathbf{q}}-\mathbf{M}_{N A}^{\prime} \underline{\tilde{\mathbf{q}}}\right)^{\top}\right\rangle \\
& =\left\langle\left(\mathbf{M}_{N A} \underline{\mathbf{q}}-\mathbf{M}_{N A}^{\prime} \mathbf{T}_{v} \underline{\mathbf{q}}\right)\left(\mathbf{M}_{N A} \underline{\mathbf{q}}-\mathbf{M}_{N A}^{\prime} \mathbf{T}_{v} \underline{\mathbf{q}}\right)^{\top}\right\rangle \\
& =\left(\mathbf{M}_{N A}-\mathbf{M}_{N A}^{\prime} \mathbf{T}_{v}\right)\left\langle\underline{\mathbf{q q}}^{\top}\right\rangle\left(\mathbf{M}_{N A}-\mathbf{M}_{N A}^{\prime} \mathbf{T}_{v}\right)^{\top}
\end{aligned}
$$

where $\left\langle\underline{\mathbf{q q}}^{\top}\right\rangle$ is the true covariance of $\underline{\mathbf{q}}$. Two choices of $\left\langle\underline{\mathbf{q q}}^{\top}\right\rangle$, chosen as examples, are described in the main text and used to estimate the nullspace uncertainty. 


\subsubsection{Inference of ML $\delta^{18} \mathrm{O}_{c}$ conditions in a four-box model}

This appendix demonstrates a relationship between pointwise resolvability and ventilation rates in a four-box model (Figure 2.9 using a single benthic $\delta^{18} \mathrm{O}_{c}$ record, NA3146. The utility of column-weighting to avoid solution artifacts is also discussed.

Model volumes and fluxes correspond in an order-of-magnitude sense to the geometry and vertical transports of the modern North Atlantic [Ganachaud and Wunsch, 2000]. Fluid with $\delta^{18} \mathrm{O}_{c}$ equal to $q_{M}(t)$ and $q_{H}(t)$ is transported at constant rates $F_{M}=1 \mathrm{~Sv}(1 \mathrm{~Sv}=$ $10^{6} \mathrm{~m}^{3} \mathrm{~s}^{-1}$ ) and $F_{H}=9 \mathrm{~Sv}$ from middle- and high-latitude ML boxes, respectively, to an intermediate box with volume $V_{1}=10^{8} \mathrm{~km}^{3}$ and $\delta^{18} \mathrm{O}_{c}$ equal to $c_{1}(t)$. The intermediate box in turn exchanges fluid at constant rate $F_{12}=10 \mathrm{~Sv}$ with a deep box with volume $V_{2}=$ $10^{8} \mathrm{~km}^{3}$ and tracer concentration $c_{2}(t)$. A compensating flux, $F_{2 \infty}=10 \mathrm{~Sv}$, evacuates fluid from the deep box out of the system.

The state vector in the four-box model is $\mathbf{c}(t)=\left[c_{1}(t), c_{2}(t)\right]^{\top}$ and the ML vector is $\mathbf{q}(t)=\left[q_{M}(t), q_{H}(t)\right]^{\top}$. Combining the equations for conservation of volume and $\delta^{18} \mathrm{O}_{c}$, the evolution in each box is described by a vector equation in the form of Eq. (2.1),

$$
\mathbf{c}(t+\Delta t)=\left[\begin{array}{cc}
1-\frac{\Delta t}{\tau_{1}} & 0 \\
\frac{F_{12} \Delta t}{V_{2}} & 1-\frac{\Delta t}{\tau_{2}}
\end{array}\right] \mathbf{c}(t)+\left[\begin{array}{cc}
\frac{F_{H} \Delta t}{V_{1}} & \frac{F_{M} \Delta t}{V_{1}} \\
0 & 0
\end{array}\right] \mathbf{q}(t)
$$

where the flushing times, $\tau_{1}$ and $\tau_{2}$, are defined as $\tau_{1}=V_{1} /\left(F_{M}+F_{H}\right)$ and $\tau_{2}=V_{2} /\left(F_{M}+F_{H}\right)$; here $\tau_{1}=\tau_{2}=317$ years. Green's functions describing $\delta^{18} \mathrm{O}_{c}$ propagation from the two ML regions to the deep box (Figure 2.9) are qualitatively similar to those derived from GH12 for the North Atlantic core site (Figure 2.3. Green's function for $q_{H}$ is $\frac{F_{H}}{F_{M}}=9$ times larger than that for $q_{M}$ at all times, a consequence of differing volume fluxes out of the two ML regions. In short, deep box $\delta^{18} \mathrm{O}_{c}$ is influenced by a sum of ML signals, $q_{M}(t)$ and $q_{H}(t)$, lagged and smoothed in time and spatially weighted, similar to the model of GH12. 
(a)

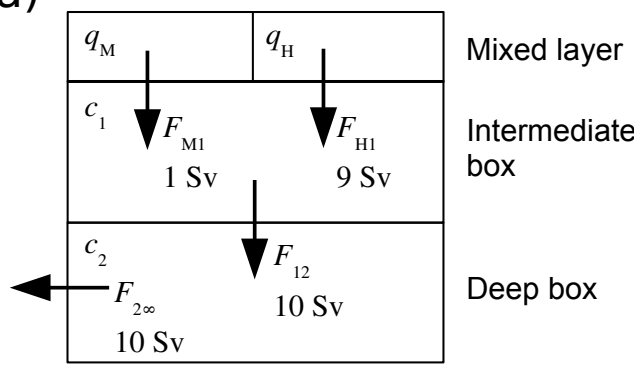

(b)

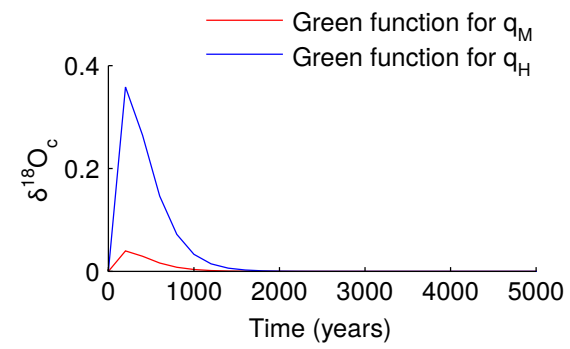

(c)

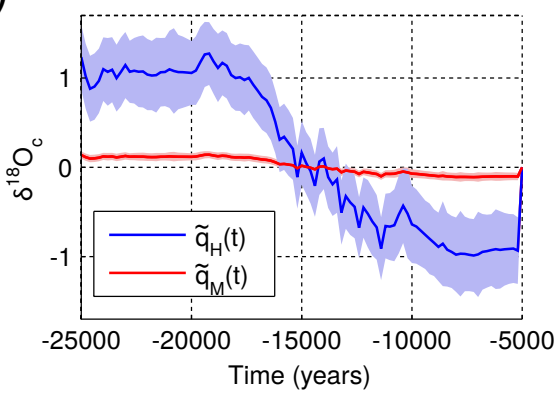

(d)

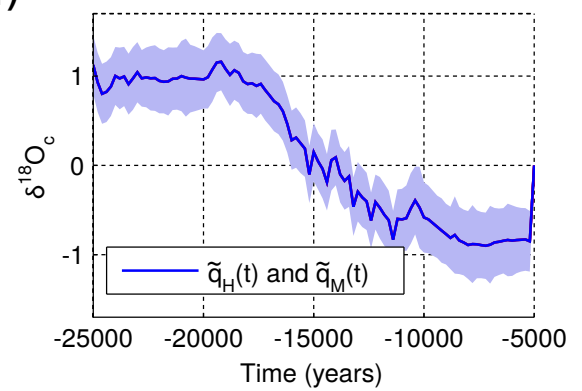

Figure 2.9: (a) Four-box tracer transport model schematic. $\delta^{18} \mathrm{O}_{c}$ values $q_{M}$ and $q_{H}$ in two ML boxes are injected into box 1 at rates $F_{M}=1 \mathrm{~Sv}$ and $F_{H}=9 \mathrm{~Sv}$. Fluid is homogenized in box 1 and flows into box 2 at a rate $F_{M}+F_{H}=10 \mathrm{~Sv}$, where it subsequently leaves the system. (b) Green's function for $q_{M}\left(q_{H}\right)$ is generated by stepping Eq. (2.26) forward in time forced by ML conditions that are zero in both boxes at all times except $q_{M}(0)=1\left(q_{H}(0)=1\right)$. (c, d) ML $\delta^{18} \mathrm{O}_{c}$ anomaly estimates and their standard error in cases without (c) and with (d) column normalization. In both cases values at $5 \mathrm{ka}$ lie in the nullspace and are 0 , as they are not constrained by benthic observations. In (d), $\tilde{q}_{M}(t)$ and $\tilde{q}_{H}(t)$ are identical. All $\delta^{18} \mathrm{O}_{c}$ values have units of permil VPDB. 
As in Section 2.4, set $t_{0}=-25000$ years, $\Delta t=200$ years, and the number of times $S=101$. Choose $\mathbf{B}=[0,1]$ in Eq. (2.2) to represent NA3146 as a noisy estimate of deep box $\delta^{18} \mathrm{O}_{c}$. The four-box inverse problem is described by Eq. 2.7, where $\underline{\mathbf{G}}$ is constructed (Appendix 2.7.1) from $\mathbf{B}$ and the matrices in Eq. 2.26, the $101 \times 1$ vector $\underline{\mathbf{y}}$ is the objectivelymapped NA3146 record, $\underline{\mathbf{n}}$ is a $101 \times 1$ vector of observational error, and $\underline{\mathbf{q}}$ is a $202 \times 1$ vector of $q_{M}$ and $q_{H}$ at all times. Effects of initial conditions are ignored. The problem is row-weighted to reflect the objectively-mapped uncertainties of NA3146 (Section 2.2).

The problem is solved with and without scaling the columns of $\underline{\mathbf{G}}$ by their norms [Wunsch, 2006]. Both the scaled and unscaled problems have $K=100$ nonzero singular values with the corresponding singular vectors describing variations in $q_{M}$ and $q_{H}$ in a fixed ratio at all times except $t=5000$ years, which is not constrained by the benthic data due to the finite lag in tracer transport. Information about differences between $q_{M}$ and $q_{H}$ is destroyed en route to box 2 by mixing in box 1; as a result, these differences, at all times, lie in the nullspace. $K^{\prime}=K=100$ is used in both solutions because all nonzero singular values are greater than the observational uncertainties.

The scaled and unscaled solutions are shown in Figure 2.9. In both cases, the solution exactly reconstructs NA3146 (not shown) except for an initial deviation due to erroneous initial conditions. The unscaled solution is biased so that the solution in each ML location is proportional to the respective volume fluxes into box 1, i.e. $\tilde{q}_{H}(t) / \tilde{q}_{M}(t)=F_{H} / F_{M}$. By contrast, the scaled solution infers the same values in the two ML regions at all times, so that $\tilde{q}_{H}(t)=\tilde{q}_{M}(t)$. Neither solution is truly desirable, but because signals from the two surface boxes are mixed en route to the observations, the data cannot "unmix" spatial gradients and the solutions, $\tilde{q}_{H}(t)$ and $\tilde{q}_{M}(t)$, can only be inferred in a fixed ratio. Without scaling the problem, that ratio is set by $F_{H} / F_{M}$, and large spatial gradients ( $a$ priori undesirable for ocean tracer distributions) in $\mathrm{ML} \delta^{18} \mathrm{O}_{c}$ may be inferred because of the distribution of surface ocean vertical velocities. The four-box model recovers two characteristic features of the problem 
solved in Section 2.4. First, scaling by column norms removes spurious spatial gradients but forces ML boxes to covary; this effect is probably responsible for the highly uncertain patterns of spatial covariance inferred in Section 2.4. Second, in the scaled case, pointwise resolvabilities in the two ML boxes are proportional to volume fluxes out of those boxes (and, by extension, to corresponding Green's function amplitudes), $r_{M}=F_{M} /\left(F_{H}+F_{M}\right)=0.1$ and $r_{H}=F_{H} /\left(F_{H}+F_{M}\right)=0.9$. This result again echoes findings in Section 2.4, though the relationship between the pointwise resolvability $r$ and ventilation rates is expected to be more complex in the many-box model of GH12. 


\section{Chapter 3}

\section{GOSE: A Glacial Ocean State Estimate constrained by LGM near-sea surface temperature proxies}

\subsection{Introduction}

Advances in data collection and computing power in recent decades have permitted increasingly sophisticated descriptions of the world ocean circulation. So-called ocean state estimates are of particular utility because they are dynamically consistent fits of ocean general circulation models (OGCMs) to observations. Because state estimates conserve mass, heat, and other physical quantities, they have proven useful for calculation of budgets, heat transports, and trends.

Extending these techniques to other geologic intervals is a promising avenue for understanding climate change [Winguth et al., 2000, Dail and Wunsch, 2014] as variability in the Earth's climate and oceans is believed to extend to all scales in time and space, but direct 
observations are limited to decadal and shorter time scales. Inference of the evolution of past climates from paleoclimate archives can improve our understanding of the physics underlying low-frequency variability in the climate system and may provide analogs for future climate change.

Paleoceanographic state estimation is complicated by the lack of direct observations. Only indirect proxy observations of past environmental conditions are available, most of which are derived from the biological remnants of ancient ocean-dwelling organisms. These observations are noisy, sparse in space and time, and may have only tenuous relationships to environmental variables. In this context, paleoceanographic state estimation is also promising because it permits using model dynamics to constrain interpretations of proxy observations and to quantify the inferential power of the data.

Several factors motivate the selection of the Last Glacial Maximum (LGM, ca. 23 - 19 thousand years ago, ka) in particular as a study subject. First, the LGM and last deglaciation are comparatively well-studied intervals in the Earth's past, and a relatively large number of observations exists to constrain physical models. Second, a preponderance of evidence suggests that the LGM conditions, which fell at the end of the last glacial period, were a persistent and dramatic excursion from the present-day climate, with large ice sheets in the Northern Hemisphere, lower sea levels, and a global mean cooling of several degrees [Clark] et al., 2012]. Beyond the scientific motivations of studying a strongly perturbed climate, the large amplitude of the glacial signal aids making inferences from noisy paleoclimate proxies, and the ability to use radiocarbon dating techniques reduces chronological uncertainty. Finally, the LGM has motivated numerous provocative hypotheses about the sensitivity of the ocean circulation and the role of carbon dioxide in the evolution of climate on centennial and longer time scales, with implications for modern climate change [e.g. Shakun et al., 2012]. Better constraints on the LGM will ultimately aid understanding of deglacial climate change, with possible implications for understanding the future evolution of modern climate. 
This chapter presents a global state estimate at the LGM, an OGCM simulation fit to the LGM NSST observations. This work expands results from Dail and Wunsch [2014] through use of a global model domain and because the state estimate duration is 100 years (as opposed to 10 years in Dail and Wunsch [2014]), more consistent with a circulation state that could have persisted for a long period of time, as has been hypothesized for the LGM.

To fit NSST observations, a seasonally quasi-steady state estimate (SQSE) is sought in which ocean state variables (velocities, densities, temperature, etc.) and model forcings (winds, air temperature, etc.) consist of repeated seasonal cycles in time. The assumption made in this chapter is that data spanning 23 - $19 \mathrm{ka}$ are taken to be representative of a single state (as opposed to the approach of Chapter 2, where interest lay in inferring transient ocean properties). Such approximations are common (often implicitly) in paleoclimate studies, where measurements nominally from the same era are taken to be contemporaneous. The ocean circulation is not expected to be steady on any time scale; nevertheless, the ill-posedness of the LGM ocean circulation inverse problem motivates the search for SQSEs as a way to extend the power of sparse observations. Making a steady assumption over the reconstruction interval greatly reduces the number of unknown model parameters, making the inverse problem less underconstrained. This is in contrast to state estimates of the modern ocean such as ECCO (Estimating the Climate and Circulation of the Ocean; Wunsch and Heimbach [2007]) which are typically time-varying, reflecting the non-steady nature of the ocean (due to both internal variability and exogenic forcing) and the ability of the growing wealth of modern observations (including from satellites, ARGO floats, and ship-based hydrography) to constraint climate transience. 


\subsection{Background}

\subsubsection{Observations and simulations of near-sea surface temperature at the LGM}

Understanding the ocean at the LGM has been a major focus of the paleoceanographic community for several decades. Most information about the LGM ocean state comes from proxy records of ocean tracers derived from sediment core records. Commonly used paleoproxy quantities include the isotope ratios $\delta^{18} \mathrm{O}, \delta^{13} \mathrm{C}$, and $\Delta^{14} \mathrm{C}$, and the elemental ratios $\mathrm{Mg} / \mathrm{Ca}$, $\mathrm{Cd} / \mathrm{Ca}$, and ${ }^{231} \mathrm{~Pa} /{ }^{230} \mathrm{Th}$. This chapter focuses on near-sea-surface temperature (NSST) proxy observations; Chapter 4 treats the generic problem of ocean state estimation using passive tracers.

NSST data used in this study are from the Multiproxy Approach for the Reconstruction of the Glacial Ocean Surface (MARGO) compilation of near sea surface temperature values and sea ice proxies from 19-23 ka (Waelbroeck et al. [2009]; Figure 3.6). Observations are taken to represent "near" sea surface temperature rather than sea surface temperature because of the variable depth ranges presumably occupied by organisms used for temperature reconstructions. The compilation includes both "transfer function" approaches, which match past abundances of planktonic foraminifera, diatoms, dinoflagellate cysts and radiolarians to modern analogues, as well as geochemical thermometers based on alkenones and planktonic foraminiferal $\mathrm{Mg} / \mathrm{Ca}$ ratios.

Waelbroeck et al. [2009] described several features in MARGO reconstructed LGMmodern anomalies (Figure 3.1) as being consistent across proxy types. In the North Atlantic, cooling in midlatitudes is stronger in the eastern than in the western basin, and the Nordic seas are ice-free during glacial summer. In the tropics, east-west gradients in cooling are observed in the Pacific, Atlantic, and Indian Oceans, including a cooling of the western Pacific warm pool. Cooling in the Southern Ocean suggests a shift in the polar front to $45^{\circ}$ 
$\mathrm{S}$ and a cooling by $2-6^{\circ}$ in austral summer. Understanding the dynamical origins of these apparently coherent signals would have implications for the LGM atmospheric state, which is closely related to the surface ocean temperature by communication of air-sea heat fluxes and momentum, and the ocean's role in transporting and storing heat.

Numerous studies have described NSSTs in forward general circulation models run under LGM conditions, including simulations from the first, second and third phases of the Paleoclimate Modeling Intercomparison Project (PMIP1, PMIP2 and PMIP3). These initiatives used standardized LGM boundary conditions (sea level, orography, greenhouse gases, and orbital parameters; see Braconnot et al. [2007]) in several coupled ocean-atmosphere and ocean-land-atmosphere climate models. Climate models typically disagree with one or more of the large-scale temperature signals observed in MARGO. For example, in the tropical oceans, Otto-Bliesner et al. [2007] found that PMIP2 models had a similar range of cooling $\left(1^{\circ}-2.4^{\circ} \mathrm{K}\right)$ to that estimated from MARGO $\left(1.7^{\circ} \pm 1^{\circ} \mathrm{K}\right)$, and that simulated Atlantic cooling was larger than in the Pacific, also in agreement with the observations. However, the large east-west gradients in tropical oceans were much less pronounced than in MARGO. The authors suggested that model biases in tropical upwelling and thermocline dynamics were at least partly to blame for the misfit. Hargreaves et al. [2011] found that simulated NSSTs in PMIP1 and PMIP2 did not give unreliable estimates of global MARGO data when the model results were treated as an ensemble; i.e., the spread in model results was comparable to observational uncertainty. However, Dail and Wunsch [2014] found that individual PMIP2 simulations did not fit MARGO data within their uncertainties in the North Atlantic, and ensemble averages reported by Braconnot et al. [2007] and individual model results from Tao et al. [2013] showed North Atlantic cooling patterns with an east-west gradient opposite that seen in the data. Ballarotta et al. [2013] compared LGM simulations at $1^{\circ}$ and $0.25^{\circ}$ horizontal resolution in the NEMO (Nucleus for European Modeling of the Ocean) OGCM and found that increasing resolution to permit the presence of eddies did not have a significant 
a

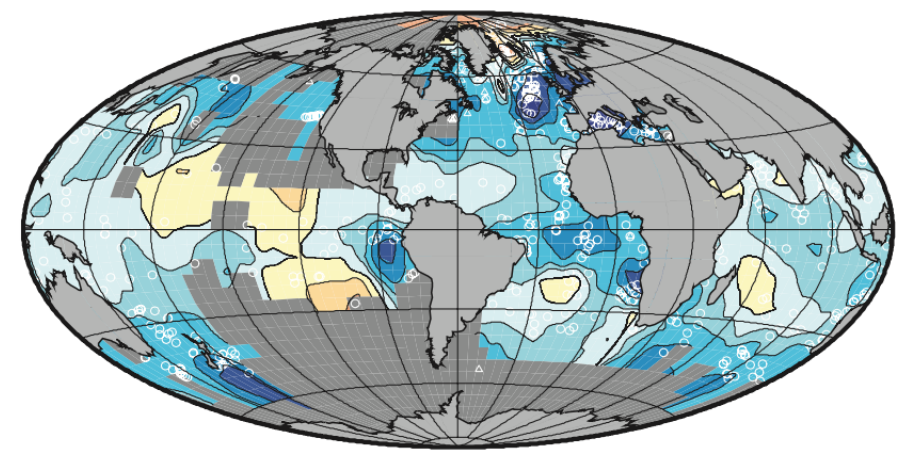

b

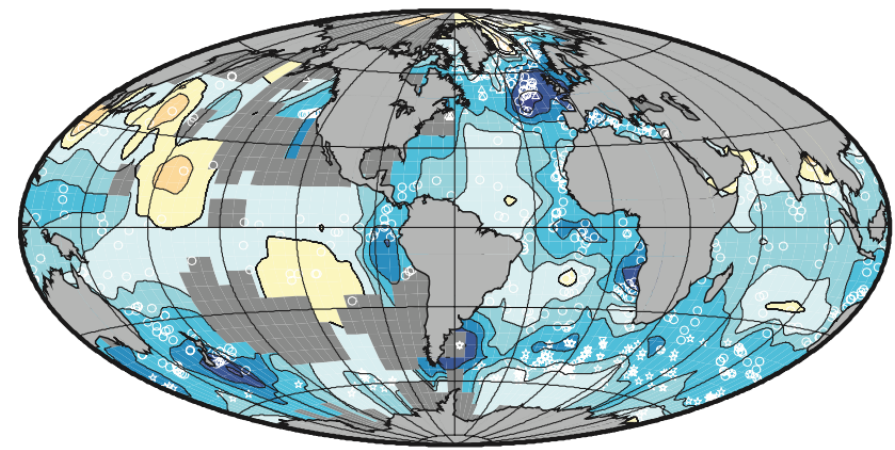

C
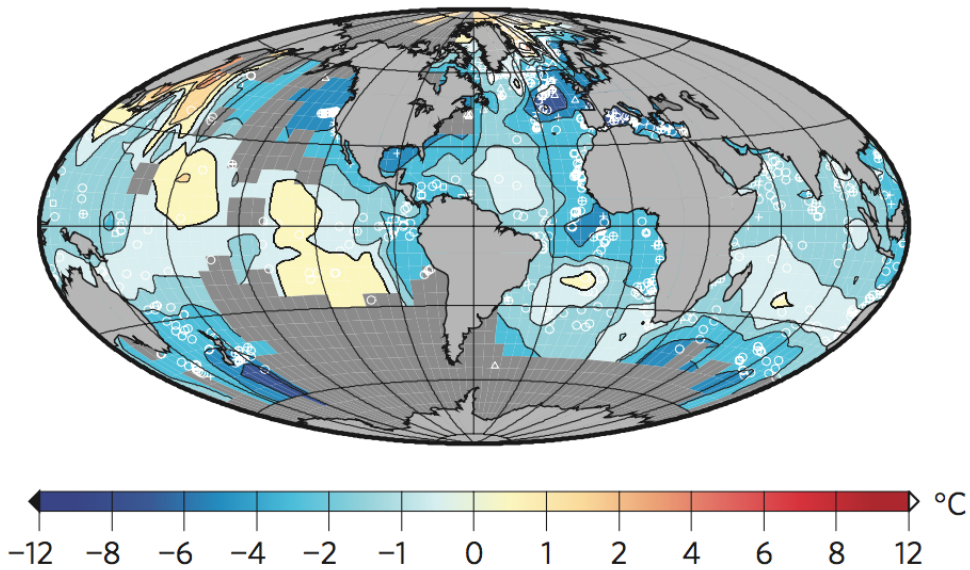

Figure 3.1: Interpolated LGM sea surface temperature anomalies from Waelbroeck et al. [2009], their Figure 2. Anomalies are computed as the difference between LGM temperature reconstructions and the World Ocean Atlas [Conkright et al., 1998]. a, July-AugustSeptember mean; b, January-February-March mean; $\mathbf{c}$, annual mean. Symbols denote proxy locations. Reprinted by permission from Macmillan Publishers Ltd: Nature Geoscience, Waelbroeck et al. [2009]. 
impact in fitting the MARGO data.

A popular metric of modern and past circulation states is the strength of the Atlantic meridional overturning circulation, which has been related to surface buoyancy transport and fluxes [e.g., Kuhlbrodt et al., 2007] and NSSTs [Delworth and Mann, 2000] and is widely used to diagnose large-scale climate changes and sensitivities despite being a problematic quantity that can obscure underlying physical processes [Wunsch and Heimbach, 2013]. Figure 3.2 shows AMOC streamfunctions in several PMIP2 simulations [Otto-Bliesner et al. 2007]. While the maximum AMOC in the modern control runs of all models fall within the observational range of $18 \pm(3-5)$ Sv [Talley et al., 2003], visual inspection shows qualitative disagreement between the modern simulations. Under LGM conditions, one of the models has a shallower and weaker AMOC; one model remains nearly the same; and two have deeper, stronger overturning cells. Some of this disagreement may be attributable to different initialization and insufficient equilibration of the ocean models [Zhang et al., 2013]. Muglia and Schmittner [2015] report that PMIP3 simulations show stronger AMOC transport (computed as the maximum streamfunction value at $25^{\circ} \mathrm{N}$ ) at the LGM than in the modern, and that all except the Community Climate System Model, version 4 (CCSM4) had deeper upper AMOC cells (defined as positive values of the overturning streamfunction). Whether the improved agreement among PMIP3 models in simulating AMOC strength is due to different equilibration or model initialization, model grids or bathymetry, model physics, or some convergence in the results brought on by the more complex models used in PMIP3, is not clear. 

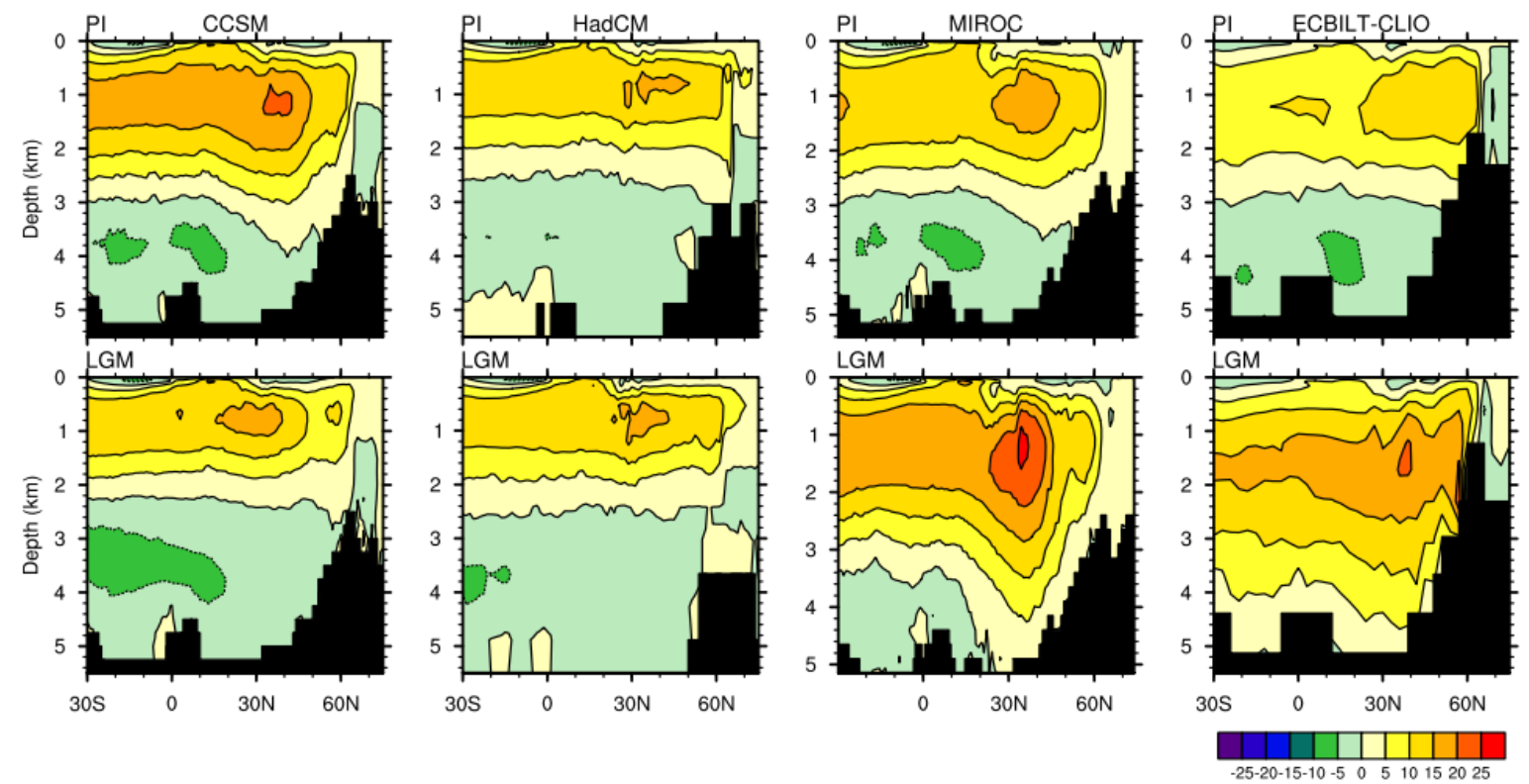

Figure 3.2: Figure 1 from Otto-Bliesner et al. [2007] showing the Atlantic Meridional Overturning Circulation (AMOC) streamfunction simulated by the PMIP2 coupled atmosphereocean general circulation models (GCMs) for modern (top) and LGM conditions (bottom). Units are in Sverdrups $\left(\mathrm{Sv}, 10^{6} \mathrm{~m}^{3} \mathrm{~s}^{-1}\right)$. A modern observational range of AMOC maxima is $18 \pm(3-5) \mathrm{Sv}$ [Talley et al., 2003]. Streamfunctions in PMIP3 are different still; see Muglia and Schmittner [2015]. 


\subsubsection{Previous inverse studies of the LGM constrained by MARGO ob- servations}

Showing how an OGCM can be adjusted to reproduce the MARGO data, or concluding that the data are in conflict with model dynamics, would be a step forward in our understanding of climate and upper-ocean circulation at the LGM. Efforts to combine models and data to constrain the ocean and climate at the LGM have been ongoing since the seminal work of LeGrand and Wunsch [1995]. Many of these studies focus on the distribution of passive tracers, especially in the abyss, and use some form of kinematic model with dynamical constraints; a review of this literature is presented in Chapter 4 . Fewer studies have focused on inferring ocean circulation from the MARGO database, in part because temperature is an active tracer (i.e., it affects tracer transport through its influence on the density field) and thus requires a more complex model to represent its distribution (e.g., Marchal et al. [2016]).

Dail [2012b] and Dail and Wunsch [2014] (hereafter DW14) obtained solutions for the circulation and climate of the LGM Atlantic by fitting the MITgcm to the MARGO data using the method of Lagrange multipliers (discussed in the next section). Their LGM solution requires only moderate changes from modern climate to fit the data, though it was necessary to double reported uncertainties for dinoflagellates and alkenones to bring the MARGO dataset into self-consistency. The resulting state estimate has higher transports in both the subtropical and subpolar gyres, a larger Gulf Stream transport, and a more zonal North Atlantic Current at the LGM. The LGM AMOC is weaker than a modern simulation (9 Sv vs $16 \mathrm{~Sv}$, measured as the maximum of the zonally averaged meridional streamfunction), though the 10-year integration time was presumably insufficient to spin up changes to the deep circulation.

Kurahashi-Nakamura et al. [2013] sought to quantify the ability of the MARGO data to infer LGM AMOC strength. Several circulation scenarios were simulated in the MITgcm through different choices of boundary conditions and internal physics, and "pseudoproxy" 
data were constructed by adding noise to model values at MARGO data locations averaged over the last ten years of each model run. The authors used the pseudoproxy data and the method of Lagrange multipliers to estimate AMOC strength in the various circulation scenarios. They found that using pseudoproxy data improved AMOC estimates in some cases, though their results were sensitive to choices of initial conditions. AMOC strengths were not found to be a robust measure of reconstruction fidelity in other variables, such as highlatitude SST fields.

These studies lay a groundwork for using MARGO to generate LGM state estimates. Key questions remain regarding physical mechanisms that might explain large-scale variations in NSST observations. Next we describe in greater depth the state estimation machinery used in this study as well as by Kurahashi-Nakamura et al. [2013] and DW14.

\subsubsection{State estimation using the method of Lagrange multipliers}

The method of Lagrange multipliers (also known as the adjoint gradient method, 4DVAR, and the Pontryagin principle) is an established method for ocean state estimation. The approach has yielded state estimates fit to modern oceanographic data through the ECCO project [e.g., Forget et al., 2015a] with a broad range of applications. A brief summary is presented below; for more detail, the reader is invited to consult discussions in Gebbie [2004], Wunsch [2006], Wunsch and Heimbach [2007], and references therein. The OGCM used is the MITgcm, an evolved form of that described by Marshall et al. [1997] and Adcroft et al. [2004] that simulates the ocean circulation under hydrostatic and Boussinesq assumptions.

Denote vectors and matrices by lower- and uppercase bold letters, respectively, and define the state vector at a time $t, \mathbf{x}(t)$, to be a complete list of the prognostic variables of an ocean circulation model (temperature, salinity, velocity, sea ice thickness, passive tracer 
concentrations, etc.). The evolution of the MITgcm in time can be written as a function of the state vector

$$
\mathbf{x}(t+\Delta t)=\mathscr{L}(\mathbf{x}(t), \mathbf{q}(t), \mathbf{u}(t)), 0 \leq t \leq t_{f}=M \Delta t
$$

where $\mathscr{L}$ is a nonlinear operator, $\Delta t$ is the discrete model time step, $M$ is a positive integer, $\mathbf{q}(t)$ is a vector of model parameters that are assumed to be known (e.g., the model bathymetry), and $\mathbf{u}(t)$ are the so-called control variables and are any elements of the problem that are taken to be unknown, such as model initial and boundary conditions. Different choices of the control variables lead to different trajectories $\mathbf{x}(t)$ of the model state.

The goal of time-dependent state estimation is to find a set of controls, $\mathbf{u}(t)$, such that the system trajectory $\mathbf{x}(t)$ agrees with available observations within their uncertainties. The estimation is performed by minimizing a scalar cost function, $J(\mathbf{u})$, with several contributions. Represent the vector of observations $\mathbf{y}(t)$ at a time $t$ as a linear combination of state vector elements,

$$
\mathbf{y}(t)=\mathbf{E}(t) \mathbf{x}(t)+\mathbf{n}(t)
$$

where $\mathbf{n}(t)$ is a vector of observational noise. Define the the cost function term $J_{d a t a}$ to be the squared, weighted model-data misfit,

$$
J_{d a t a}=\frac{1}{M N} \sum_{m=1}^{M}(\mathbf{y}(t)-\mathbf{E}(t) \mathbf{x}(t))^{\top} \mathbf{R}^{-1}(t)(\mathbf{y}(t)-\mathbf{E}(t) \mathbf{x}(t))
$$

where $t=m \Delta t, M$ is the number of time steps over which the misfit is computed, $N$ is the number of observations, and $\mathbf{R}=\left\langle\mathbf{n} \mathbf{n}^{\top}\right\rangle$ is the covariance matrix of the observational noise. Adding a cost contribution of the form

$$
J_{\text {model }}=-2 \sum_{m=0}^{M-1} \mu(\mathbf{t})^{\top}[\mathbf{x}(t)-\mathbf{L}(\mathbf{x}(t-\Delta t), \mathbf{q}(t-\Delta t), \mathbf{u}(t-\Delta t))], t=m \Delta t
$$


where $\mu(t)$ is a vector of Lagrange multipliers, serves to impose the model equations upon the solution. Finally, in order to constrain the magnitude and spatial variability of inferred control variables, a third cost function term is introduced,

$$
J_{c t r l}=\sum_{m=0}^{M-1} \mathbf{u}(t)^{\top} \mathbf{Q}(t)^{-1} \mathbf{u}(t), t=m \Delta t
$$

where $\mathbf{Q}$ is the prior spatial covariance of the control variables.

Minimization of the total cost function,

$$
J=J_{\text {data }}+J_{c t r l}+J_{\text {model }}
$$

is a problem of constrained optimization, whereby the model-data misfit is reduced through a choice of controls and the state vector evolution is forced to exactly obey the dynamical constraints in $\mathscr{L}$. For low-dimensional problems, minima of $J$ can be found analytically by setting the derivatives $\partial J / \partial \mathbf{u}=0$ and solving the resulting equations. In the context of ocean modeling, the size of the state space (typically millions of state variables) and complexity of $\mathscr{L}$ (specified in the MITgcm by many thousands of lines of FORTRAN code) do not permit an analytical solution. Instead, an iterative procedure is used to compute the direction, $\partial J / \partial \mathbf{u}$, of maximally decreasing cost for infinitesimal perturbations about a particular location in the control vector space by automatic differentiation of the MITgcm (Giering and Kaminski [1998]). The controls are adjusted in the direction of locally steepest descent using a quasi-Newton algorithm [Gilbert and Lemaréchal, 1989], the cost function and local sensitivities recomputed, and the procedure repeated until a choice of controls, $\tilde{\mathbf{u}}(t)$, has decreased the total cost function so that $J_{\text {data }} \approx 1$, at which point the state estimate is considered complete.

In contrast to time-dependent state estimation, seasonally quasi-steady state estimation seeks to find time series of state vectors spanning a one-year period (a climatology) and 
satisfying dynamical, observational, and equilibration constraints. Here we discuss the example of a monthly climatology, though the resolution in time can vary depending on the application. Define the climatology $\underline{\mathbf{x}}_{i}$ to be the vector of monthly average seasonal cycle state values in the $i^{\text {th }}$ year of a simulation,

$$
\underline{\mathbf{x}}_{i}=\left[{\overline{\mathbf{x}\left(t \in \operatorname{Jan}_{i}\right)}}^{\top},{\overline{\mathbf{x}\left(t \in \mathrm{Feb}_{i}\right)}}^{\top}, \quad \ldots, \overline{\mathbf{x}\left(t \in \mathrm{Dec}_{i}\right)}\right]^{\top} .
$$

A climatology $\underline{\tilde{\mathbf{x}}}_{i}$ is a seasonally quasi-steady state estimate (SQSE) if it satisfying the following constraints:

$$
\begin{aligned}
\mathbf{x}(t+\Delta t) & =\mathscr{L}\left(\mathbf{x}(t), \mathbf{q}(t), \mathbf{u}_{c}(t)\right), t_{q e 0} \leq t \leq t_{q e f} \\
\mathbf{y} & =\mathbf{E}_{\underline{\mathbf{x}}_{i}}+\mathbf{n} \\
\underline{\tilde{\mathbf{x}}}_{i} & \approx \underline{\mathbf{x}}_{j} \forall i, j \in R_{q e}
\end{aligned}
$$

where $t_{q e 0}$ and $t_{q e 1}$ are initial and final times of a quasi-equilibrated model simulation, $R_{q e}$ is the set of calendar years spanned by those times, and $\mathbf{u}_{c}(t)$ is a climatological control vector that repeats every year. By minimizing a cost function having the form of Equation (3.6) but constrained to fit Equations (3.8)-(3.10) rather than (3.1) and (3.2), one seeks a state that obeys the model equations forced by climatological controls, can fit the data as a linear function of the monthly climatological state vector, and is approximately steady from year to year over a time duration $\tau_{e q}=t_{q e 1}-t_{q e 0}$. How long $\tau_{e q}$ should be depends on the duration deemed necessary to demonstrate quasi-steady behavior, and exactly what is meant by $\underline{\mathbf{x}}_{i} \approx \underline{\mathbf{x}}_{j}$ depends on how much drift one is willing to accept in deciding whether a model is in quasi-equilibrium.

There is no guarantee that an ocean model (let alone the real climate system, which has variability on all spatial and temporal scales) has a seasonally steady state equilibrium. The 
search for quasi-steady circulation states biases the inverse procedure against states with variability on time scales longer than the seasonal cycle that may fit the data better and be closer to the true state, though the available data do not permit inference of (for example) interannual variability. Any quasi-equilibrium ocean state estimate should be viewed as an approximate fit of a steady model to observations of a fundamentally transient system.

\subsection{Inverse modeling setup}

\subsubsection{The MITgem}

The LGM MITgcm configuration runs on a subsampled version of the ECCO version 4 grid [Forget et al., 2015a, hereafter ECCO v4], with roughly 2 degree horizontal resolution telescoping to higher resolution at the equator and poles and 50 vertical levels with thickness varying between 10 meters at the surface and 456 meters at 5900 meters depth. The lower resolution permits longer simulation times, at the expense of inferior representation of some ocean features, especially boundary currents. A model runtime of 100 years was chosen as a compromise to reduce computational time; this duration is a tenfold increase in the integration period used by DW14. The MITgcm is coupled to a viscous plastic dynamicthermodynamic sea ice model [Losch et al., 2010] subject to the same atmospheric forcings as the ocean model. Air-sea fluxes are computed using the bulk formulae of Large and Yeager [2004]. No salinity or temperature relaxation is used, but net changes in evaporation, precipitation, and runoff are compensated at every time step by adding or subtracting a uniform salinity from the entire model domain in order to prevent drifts in ocean mean salinity.

Model bathymetry (Figure 3.3) was constructed by subsampling the ECCO v4 bathymetry (itself a smoothed and subsampled version of the bathymetry described in Smith and Sandwell [1997]) and adding the anomaly between the $21 \mathrm{ka}$ and $0 \mathrm{ka}$ ICE-5G bathymetry reconstruc- 

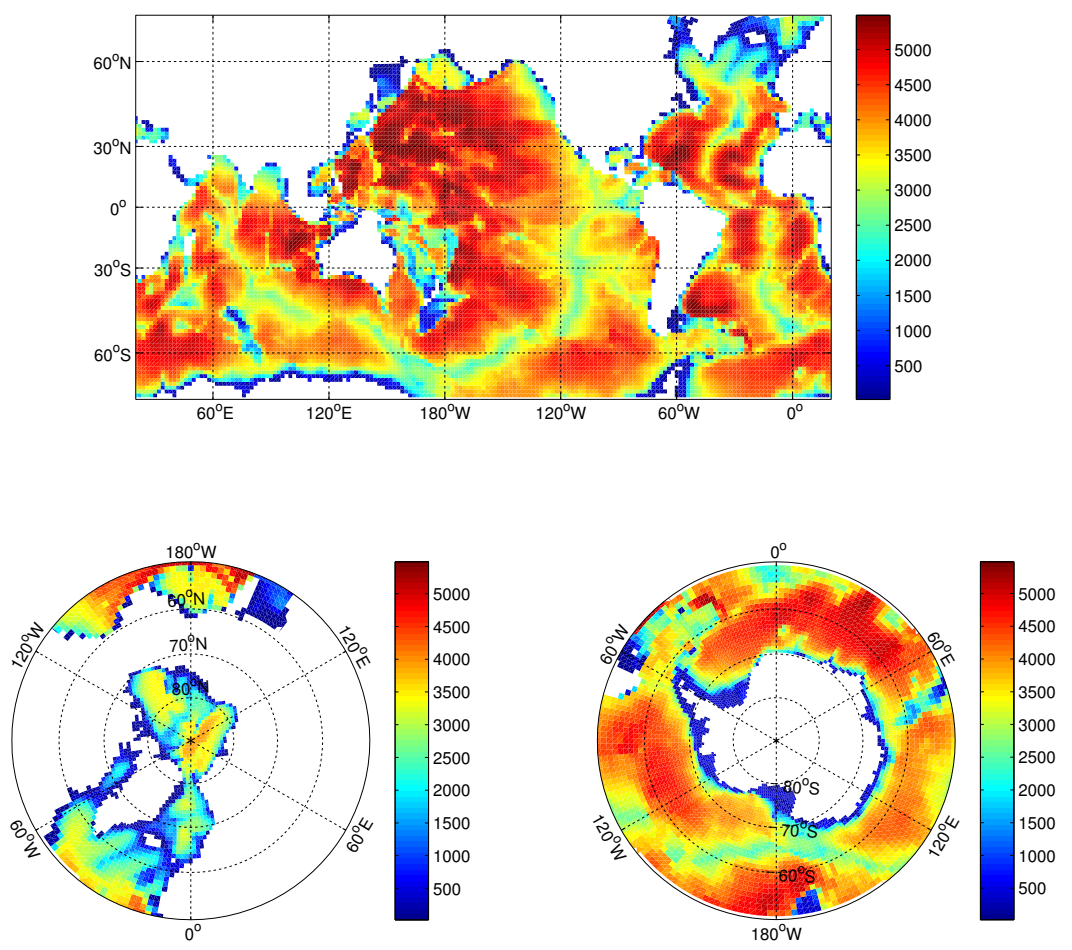

Figure 3.3: LGM bathymetry used in the state estimate in meters.

tions [Peltier, 2004]. The median LGM-modern depth anomaly is roughly 130 meters, with spatial deviations due to flexural changes in Earth's crust from ice sheet loading. A seasonal climatology of runoff is derived from Fekete et al. [2002], with runoff on the European continent between $50^{\circ}$ and $72^{\circ} \mathrm{N}$ rerouted at the latitude of the English Channel, reflecting the reconstruction of Alkama et al. [2006]. Sea ice parameters used are the same as in ECCO $\mathrm{v} 4$ except that sea ice and snow albedos were reduced by roughly $30 \%$ and sea ice strength [Weeks and Ackley, 1986] is reduced to 10,000 Pa; these changes sufficed to prevent monotonic growth in Arctic sea ice thickness that was approximately linear in time and yielded up to 100 -meter-thick sea ice after 100 years in test simulations. While there are suggestions that Arctic sea ice may have been very thick during past climate states [e.g., Hughes et al. 1977, Bradley and England, 2008], we deliberately preclude this possibility because sea ice growth contributes to ocean transience and because the sea ice model has not been tested for ice thicknesses of more than order $10 \mathrm{~m}$. 


\subsubsection{First guess control variables and prior covariances}

Choices of which model parameters to use in the vector $\mathbf{u}$ of adjustable controls (Equation (3.1), the "first guesses" of control values, and the covariances specifying how controls may be adjusted can all have a large influence on the final state estimate. Here we use the same control vector variables used in ECCO v4 - which sufficed to fit the MITgcm to a database of modern oceanographic observations - though here, as our objective is a seasonally quasisteady state estimate, control adjustments are only allowed in the monthly climatology of atmospheric variables. Omission of other model parameters from the control vector (e.g., the model bathymetry, sea ice albedo, or run length) is tantamount to assuming that those quantities are known and introduces biases into the inverse solution that are not quantified here; see Forget et al. [2015a] and references therein for a discussion of the types of error affecting ocean models.

"First guesses" of control parameters (listed in Table 3.1) specify the prior ocean state before controls are adjusted to fit the data. For underconstrained problems such as paleoceanographic state estimation, the available data are unlikely to constrain the ocean state uniquely, and the choice of first guess control parameters will influence the final state estimate. Here we choose atmospheric forcings constructed from ECCO v4 adjusted fields plus LGM minus pre-industrial (LGM-PI) anomalies computed from simulations of the CCSM4.

The ECCO v4 fields consist of 6-hourly ERA interim reanalysis fields [Dee et al., 2011] with additional control adjustments inferred using the method of Lagrange multipliers to fit a diverse modern data set; see Forget et al. [2015a] for more details. The CCSM4 consists of coupled ocean, atmosphere, land, and sea ice models, each having nominal $1^{\circ}$ horizontal resolution. The PI simulation [Gent et al., 2011] follows protocols for the fifth phase of the Climate Model Intercomparison Project (CMIP5) and uses atmospheric $\mathrm{CO}_{2}$ concentrations estimated for the year 1850 in a 1300 year simulation. The LGM simulation [Brady et al. 


\begin{tabular}{|c|c|c|c|}
\hline Control variable & $\sigma$ & First guess & Period/duration \\
\hline "SW radiation & $20 \mathrm{Wm}^{-2}$ & 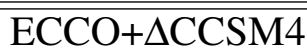 & Monthly climatology \\
\hline LW radiation & $20 \mathrm{Wm}^{-2}$ & $\mathrm{ECCO}+\Delta \mathrm{CCSM} 4$ & Monthly climatology \\
\hline Humidity & $2 \times 10^{-3}$ & $\mathrm{ECCO}+\Delta \mathrm{CCSM} 4$ & Monthly climatology \\
\hline Precipitation & $4 \times 10^{-8}$ & $\mathrm{ECCO}+\Delta \mathrm{CCSM} 4$ & Monthly climatology \\
\hline $2 \mathrm{~m}$ air temperature & $4^{\circ} \mathrm{K}$ & $\mathrm{ECCO}+\Delta \mathrm{CCSM} 4$ & Monthly climatology \\
\hline Zonal wind stress & $0.1 \mathrm{~Pa}$ & $\mathrm{ECCO}+\Delta \mathrm{CCSM} 4$ & Monthly climatology \\
\hline Meridional wind stress & $0.1 \mathrm{~Pa}$ & $\mathrm{ECCO}+\Delta \mathrm{CCSM} 4$ & Monthly climatology \\
\hline$\kappa_{d}$ & $10^{-4} \mathrm{~m}^{2} \mathrm{~s}^{-1}$ & ECCO & Constant \\
\hline$\kappa_{\sigma}$ & $500 \mathrm{~m}^{2} \mathrm{~s}^{-1}$ & ECCO & Constant \\
\hline$\kappa_{G M}$ & $500 \mathrm{~m}^{2} \mathrm{~s}^{-1}$ & $\mathrm{ECCO}$ & Constant \\
\hline Initial temperature & $3^{\circ} \mathrm{K}$ & Spin-up run & Initial \\
\hline Initial salinity & $1 \mathrm{~g} \mathrm{~kg}^{-1}$ & Spin-up run & Initial \\
\hline
\end{tabular}

Table 3.1: Control variables, prior control adjustment uncertainties $(\sigma)$, sources of first guesses, and period and duration for control elements used in the LGM state estimate. ECCO refers to the ECCO version 4 simulation in the year 2007, and averaged in space to be used on the lower-resolution llc45 grid. $\triangle \mathrm{CCSM} 4$ refers to the LGM minus pre-industrial control runs in coupled CCSM4 simulations illustrated in Figure 3.4. $\kappa_{\sigma}, \kappa_{d}$, and $\kappa_{G M}$ refer to coefficients of isopycnal diffusivity [Redi, 1982], diapycnal diffusivity, and eddy bolus velocity [Gent and McWilliams, 1990]. "Spin-up run" refers to the long forward simulation under $\mathrm{ECCO}+\triangle \mathrm{CCSM} 4$ forcings described in Section 3.4.1. 
2013] followed PMIP3 protocols, including using greenhouse gas concentrations estimated from ice cores, initializing simulations with Northern Hemisphere ice sheets, and reducing global sea level. Anomalies were added (rather than using the simulated LGM fields directly) in an effort to eliminate model biases that might be present in both the PI and the LGM CCSM4 simulations. Initial temperature and salinity distributions were generated by a 4900-year integration under first-guess conditions initialized from modern hydrography to minimize drift in the final solution.

LGM minus PI atmospheric quantities are plotted in Figure 3.4. Large changes in wind stress largely reflect orographic changes due to the presence of Northern Hemisphere ice sheets. Two-meter air temperatures are everywhere reduced, with especially pronounced cooling in the subpolar North Atlantic, Southern Ocean, and North Pacific. Downwelling longwave radiation and humidity are also uniformly lower at the LGM, likely reflecting changes atmospheric heat content and the reduced capacity of colder air to hold moisture. Patterns of precipitation and shortwave downwelling radiation are more complex, reflecting behavior of the model atmospheric circulation and cloud distribution as well as changes in Earth's orbital configuration. First guess forcing fields (the sum of CCSM4 anomalies and ECCO v4 adjusted fields) are shown in Figure 3.5. In several cases the anomalies have the same order of magnitude as the ECCO v4 fields, so that the first guess forcings constitute a substantial deviation from the modern climate.

The choice to begin with an LGM-like ocean state diverges from the approach of DW14, who used modern climatologies as the first guess and obtained a solution that diverged from the modern circulation only where necessary to accommodate observations. One rationale for our approach is that coupled model LGM simulations consistently reproduce some features (such as widespread cooling and drying and changing in wind stress due to ice sheet orography) that are desirable in a glacial state estimate. If some of these features are removed in the process of adjusting controls to fit observations, then perhaps those features are 


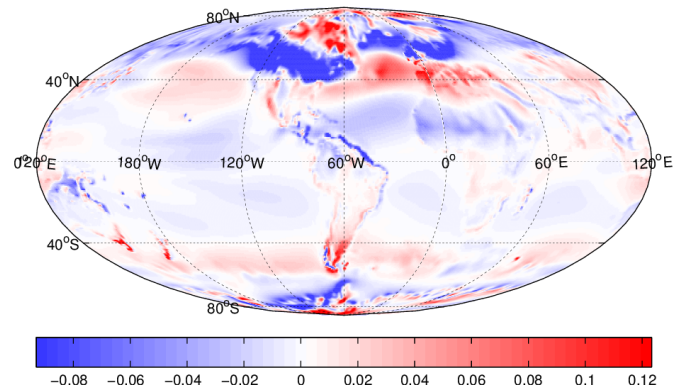

(a) Zonal wind stress anomaly $(\mathrm{Pa})$

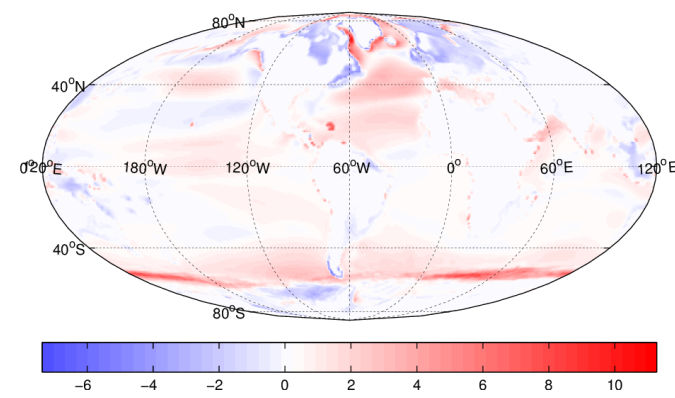

(c) Wind speed anomaly $\left(\mathrm{m} \mathrm{s}^{-1}\right)$

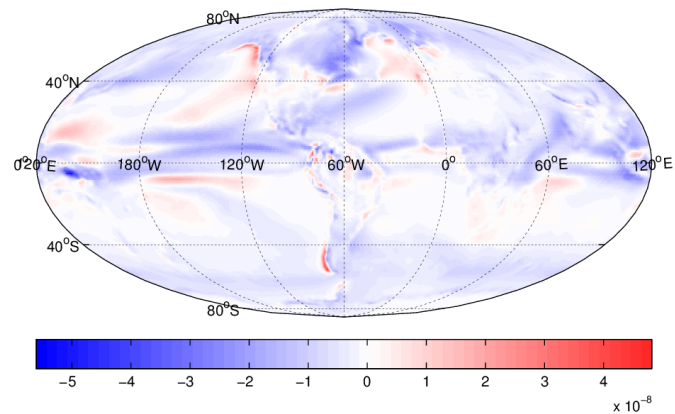

(e) Precipitation anomaly $\left(\mathrm{m} \mathrm{s}^{-1}\right)$

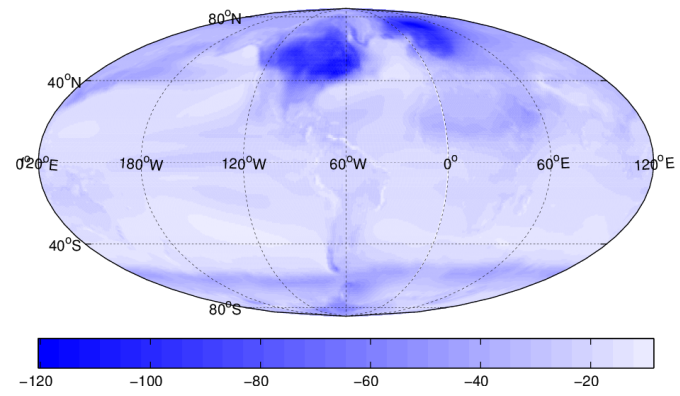

(g) Longwave radiation anomaly $\left(\mathrm{W} \mathrm{m}^{-2}\right)$

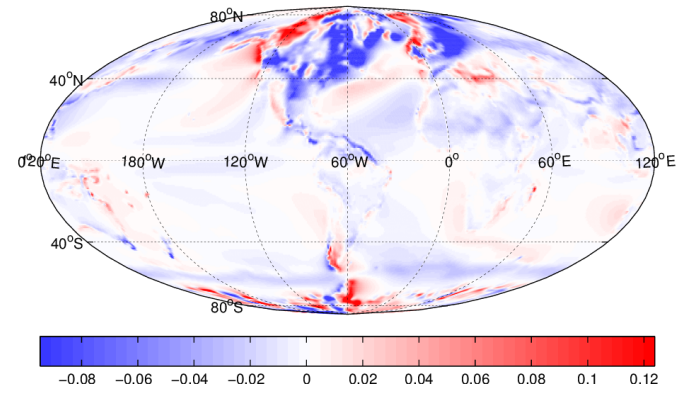

(b) Meridional wind stress anomaly $(\mathrm{Pa})$

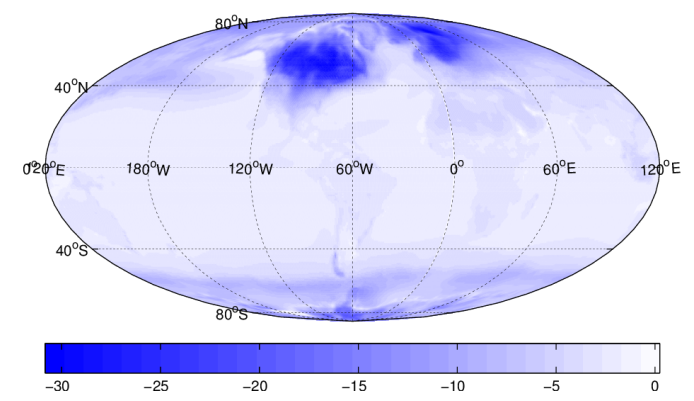

(d) Air temperature at $2 \mathrm{~m}$ anomaly $\left({ }^{\circ} \mathrm{K}\right)$

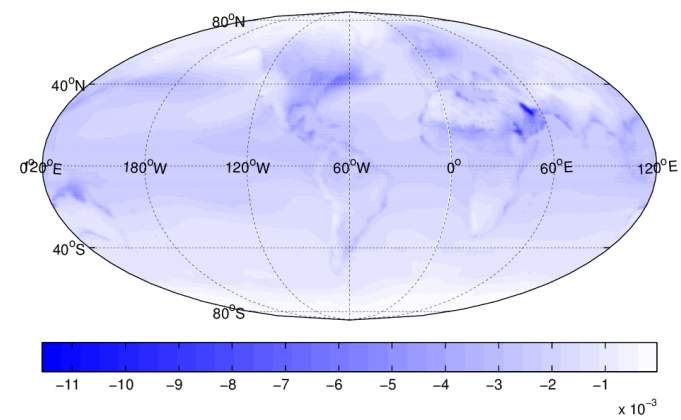

(f) Specific humidity anomaly (unitless)

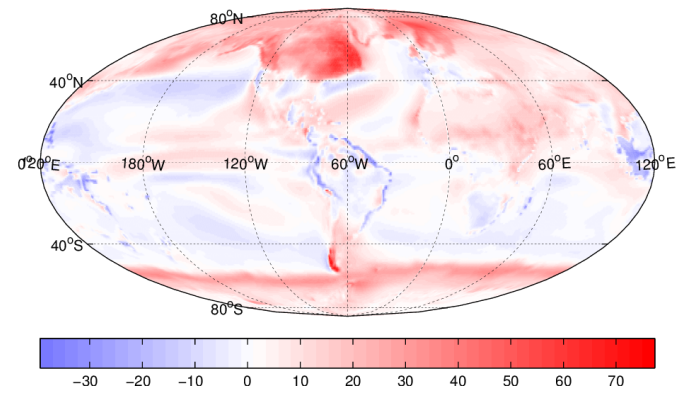

(h) Shortwave radiation anomaly $\left(\mathrm{W} \mathrm{m}^{-2}\right)$

Figure 3.4: LGM minus preindustrial quantities derived from the CCSM4 LGM PMIP3 simulation [Brady et al., 2013]. 


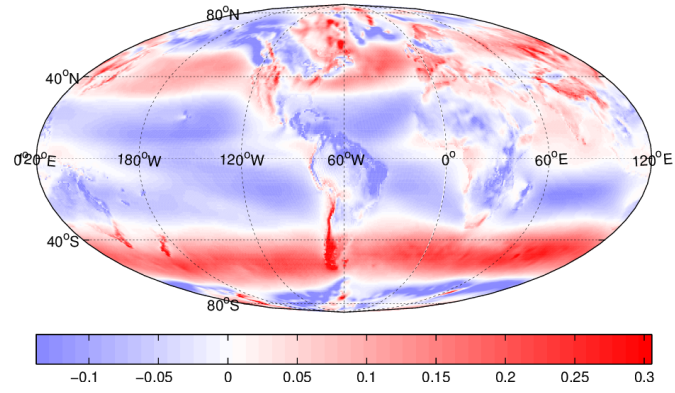

(a) Zonal wind stress (Pa)

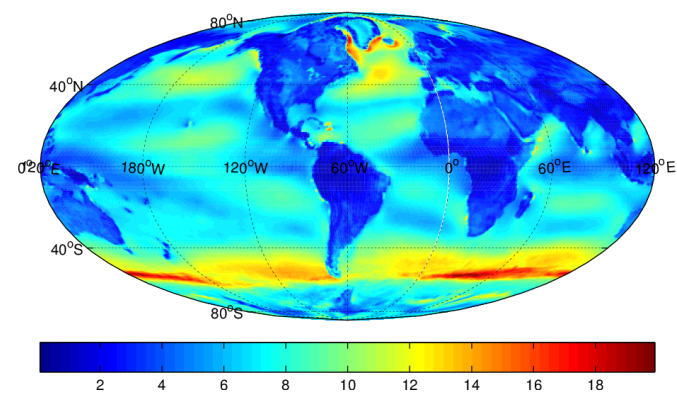

(c) Wind speed $\left(\mathrm{m} \mathrm{s}^{-1}\right)$

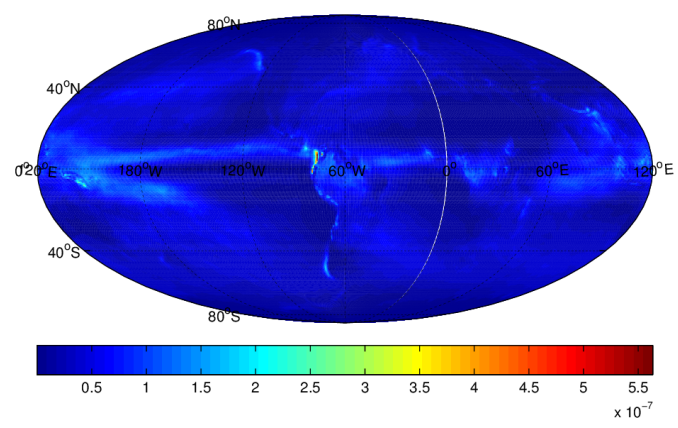

(e) Precipitation $\left(\mathrm{m} \mathrm{s}^{-1}\right)$

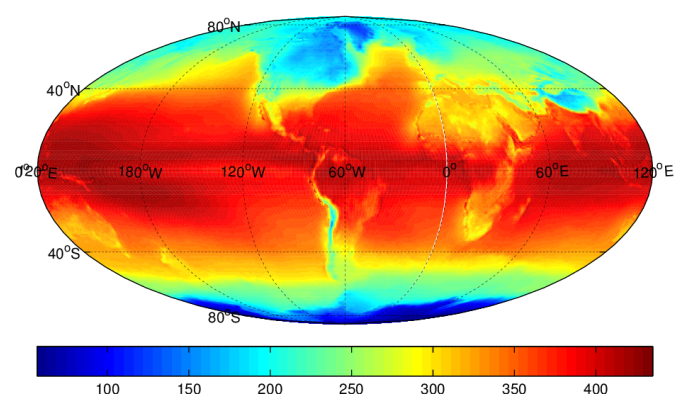

(g) Longwave radiation $\left(\mathrm{W} \mathrm{m}^{-2}\right)$

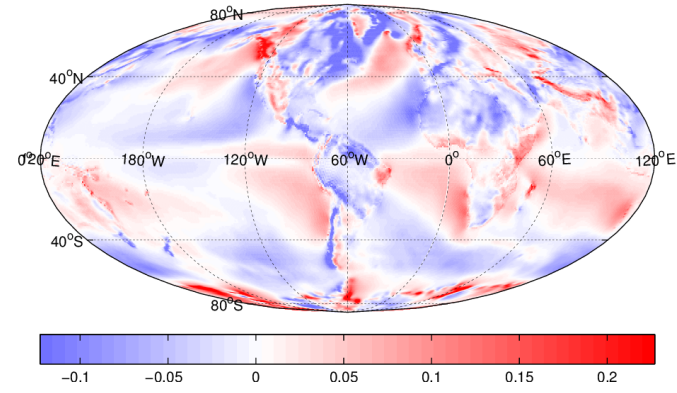

(b) Meridional wind stress (Pa)

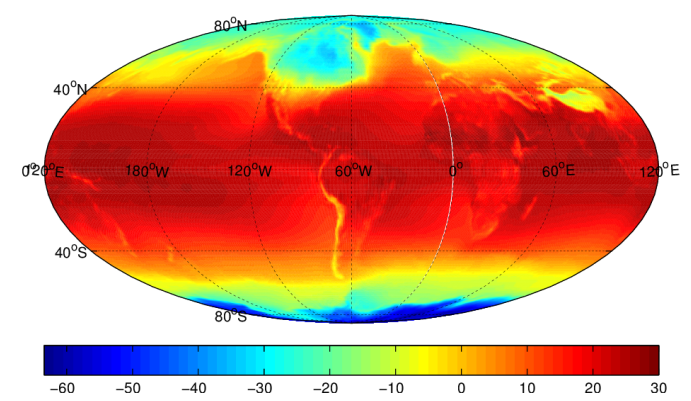

(d) Air temperature at $2 \mathrm{~m}\left({ }^{\circ} \mathrm{K}\right)$

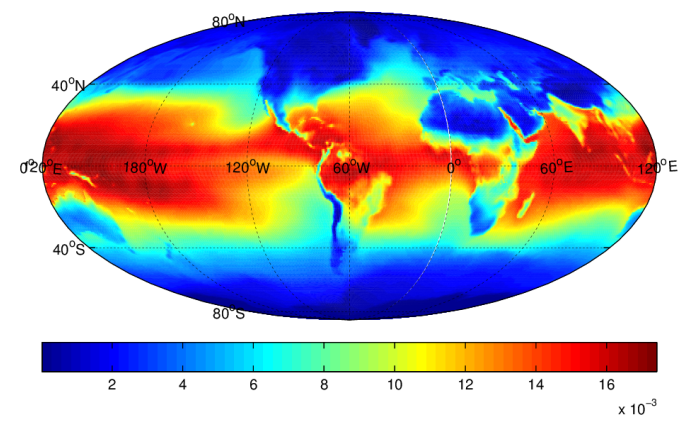

(f) Specific humidity (unitless)

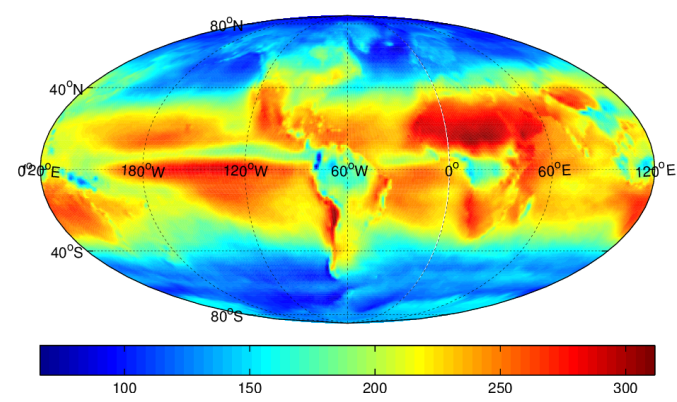

(h) Shortwave radiation $\left(\mathrm{W} \mathrm{m}^{-2}\right)$

Figure 3.5: First guess forcings constructed by adding the CCSM4 LGM minus pre-industrial quantities (Figure 3.4, Brady et al. [2013]) to the ECCO v4 adjusted forcings [Forget et al., 2015a. 
misrepresented in the model. A second, more practical rationale is that the CCSM4 LGM PI anomalies are highly coherent in space and time, reflecting in part dynamical constraints in the atmospheric components of that model. Because it is not possible to include this spatiotemporal information in the state estimation framework as currently configured, these patterns are instead introduced in the first guess. The two approaches can be seen as complementary: DW14 clearly illustrate limitations of the data, whereas here we make explicit use of prior information from modeling studies.

Prior standard deviations $(\sigma)$ of control variables are listed in Table 3.1. Following DW14, values for shortwave and longwave downwelling radiation, humidity, and precipitation are twice those used in $\mathrm{ECCO} v 4$, and $\sigma$ for temperature is four times that in ECCO v4. Standard deviations for wind stress were chosen to be $0.1 \mathrm{~Pa}$, reflecting the amplitude of CCSM4 LGM-PI wind stress changes. Standard deviations for turbulent transport parameters were taken from ECCO v4. As noted above, a limitation of the existing ECCO framework is that prior covariances can be specified only by a single standard deviation, $\sigma$, meaning that most prior information about how variables vary in space or time or with one another cannot be included in the state estimate. (An exception is the MITgcm "smooth" package, which uses the method of Weaver and Courtier [2001] to smooth inferred control changes isotropically in space to avoid grid-scale variations. The smooth package was used in ECCO v4 with a horizontal scale of 3 grid boxes; here, as we are using a lower-resolution model, smoothing is not used.)

\subsubsection{Construction of the cost function}

The $J_{\text {data }}$ term in the cost function (Equation (3.3) ) is constructed using the $5^{\circ} \times 5^{\circ}$ gridded product provided by Waelbroeck et al. [2009], which weights grid box means and uncertainties based on data type, numbers of observations available during the time period, instru- 


\begin{tabular}{|c|c|c|}
\hline Cost function term & Period/Duration & $\sigma$ \\
\hline \hline MARGO annual average & Annual average/All years & Figure 3.6 \\
\hline MARGO Jan-Feb-Mar average & Monthly average/All years & Figure 3.6 \\
\hline MARGO Jul-Aug-Sep average & Annual average/All years & Figure 3.6 \\
\hline Deviation of annual-mean T from 100-yr mean & Annual average/All years & $1 \mathrm{~K}$ \\
\hline Deviation of annual-mean S from 100-yr mean & Annual average/All years & $1 \mathrm{~g} / \mathrm{kg}$ \\
\hline
\end{tabular}

Table 3.2: Contributions to the the cost function in the LGM state estimate.

ment error, and other factors. Reflecting seasonal effects in the different proxies used (e.g., growing season), the MARGO gridded data are divided into three subsets intended to represent annual, January-February-March, and July-August-September time averages. As such, $J_{\text {data }}$ has three terms corresponding to the annual, Jan-Feb-March (JFM) and July-AugustSeptember (JAS) average proxy reconstructions. Deviations in model annual-average temperature from annual-average observations in the uppermost 310 -meter grid boxes of the model are penalized at every model year. Deviations from JAS and JFM data are penalized month-by-month in all of the corresponding months, also in the top three layers. Uncertainties used to weight the cost function are shown in Figure 3.6

An additional model drift cost function term, $J_{d r i f t}$, is penalized by computing the 100 year average (i.e., over the entire run length) of temperature and salinity at every grid box and adding the squared differences of each annual mean about the 100-year mean to the cost function. Squared misfits are scaled so that the expected standard deviations of interannual variability about the mean is $1 \mathrm{~g} / \mathrm{kg}$ and 1 degree Kelvin.

\subsection{Results}

This section quantifies improvements in the fit of the LGM MITgcm configuration to the MARGO data made using the adjoint machinery and describes features of the best-fit solution. The focus is on the 9th and 10th iterations of the adjoint; the tenth iteration is taken to be the final solution and is labeled $\overline{\mathbf{x}}_{M A R G O}$. In those solutions, model-data misfits remain, 


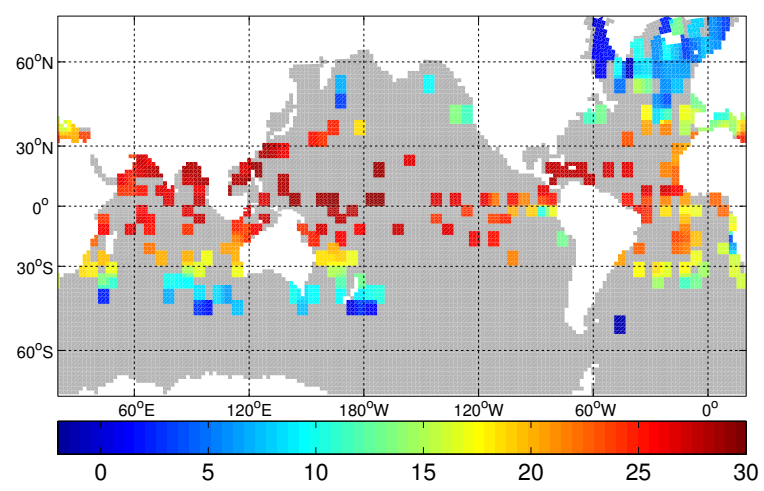

(a) July-Aug-Sep average NSST $\left({ }^{\circ} \mathrm{C}\right)$

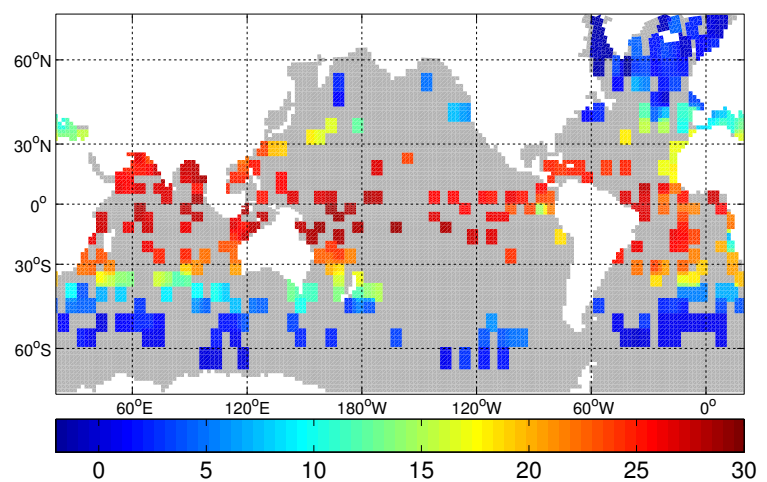

(c) Jan-Feb-March average NSST $\left({ }^{\circ} \mathrm{C}\right)$

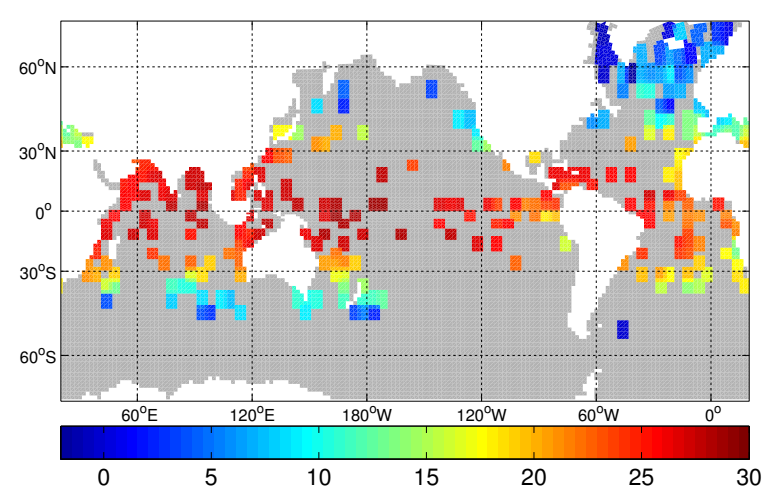

(e) Annual average $\operatorname{NSST}\left({ }^{\circ} \mathrm{C}\right)$

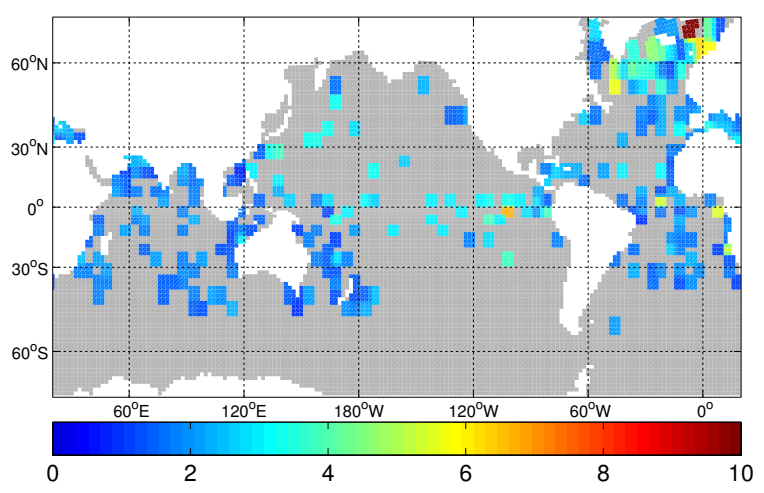

(b) July-Aug-Sep NSST uncertainty $\left({ }^{\circ} \mathrm{C}\right)$

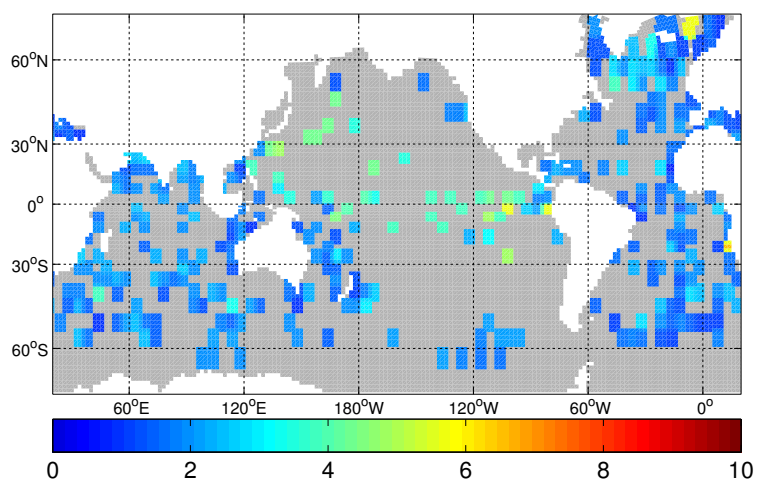

(d) Jan-Feb-March NSST uncertainty $\left({ }^{\circ} \mathrm{C}\right)$

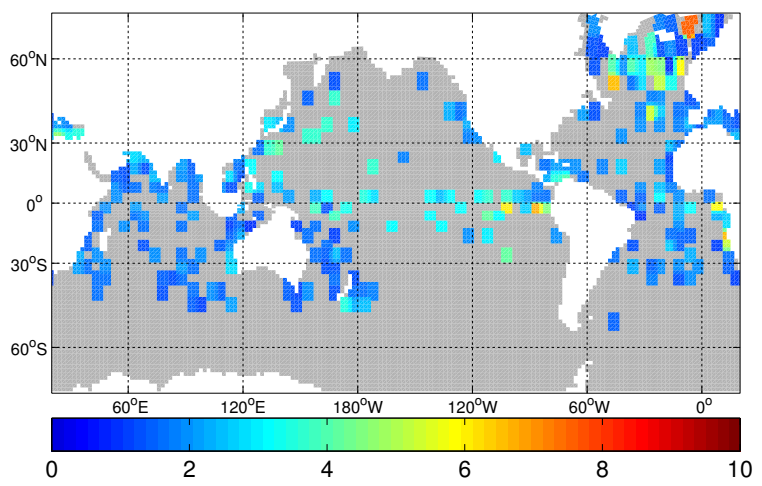

(f) Annual average NSST uncertainty $\left({ }^{\circ} \mathrm{C}\right)$

Figure 3.6: MARGO $5^{\circ} \times 5^{\circ}$ gridded temperature data and uncertainties from Waelbroeck et al. [2009] interpolated to the model grid. 
though they are of similar magnitude to those obtained in the final solution of DW14 for the North Atlantic, and could be deemed acceptable if the MARGO uncertainty estimates were increased in JFM. After more iterations it is likely that the misfit could be further reduced, at the risk of overfitting annual average and JAS observations. We are able to identify features of the state estimate and control variables that are expected to be robust under additional iterations.

\subsubsection{A quasi-equilibrated MITgem simulation forced by CCSM4 bound- ary conditions}

The initial ocean state used in the inverse procedure ("iteration 0") is obtained from the last year of a 4900-year simulation initialized with the ECCO v4 solution (year 2007) after adding $1 \mathrm{~g} / \mathrm{kg}$ to salinity at every grid box and forced by LGM first-guess atmospheric forcings (Table 3.1, including the ECCO v4 turbulent mixing parameters). This spin-up period is characterized by strong initial transients in diagnostics of tracer and mass transports, followed by a gradual approach to apparent equilibrium values (not shown). Using this quasi-equilibrated state as a starting place (rather than, e.g., the modern state, as was done by DW14) reduces model drift and equilibrates regions of the deep ocean (which might not otherwise be strongly influenced by model adjustments given the 100-year simulations used) to an estimate of LGM atmospheric conditions.

Iteration 0 conditions are not drastically different from the modern ECCO estimate. Firstguess temperatures at $5 \mathrm{~m}$ (Figure 3.7) are colder at the poles and show a substantial east-west gradient in the Pacific Ocean. The Atlantic meridional streamfunction is somewhat shallower and stronger, with a stronger upper cell, similar to the LGM-preindustrial difference diagnosed in CCSM4 [Brady et al., 2013] but at odds with several other PMIP3 simulations that show streamfunctions that are deeper and stronger [Muglia and Schmittner, 2015] and with 
scaling arguments suggesting that upper cell AMOC strength should be proportional to depth [Gnanadesikan, 1999]. Transport in the ACC is roughly $10 \mathrm{~Sv}$ larger than in ECCO; CCSM4 exhibits an even larger increase at the LGM. Transport in the Florida Straits ( 12 Sv) is substantially lower than in ECCO ( $\sim 30 \mathrm{~Sv})$ and modern estimates, possibly owing to a bias due to lower resolution; examination of the barotropic streamfunction (not shown) shows that the overall pattern of flow is similar in the two estimates, suggesting that more transport feeding the Gulf Stream originates east of the model Caribbean. In addition to forcing changes, differences between first-guess conditions and ECCO are also expected to arise because of changes in bathymetry, the coarsening of the model grid, and the long duration of the simulation; here we argue that this quasi-equilibrated simulation is a reasonable starting place for fitting the data. 

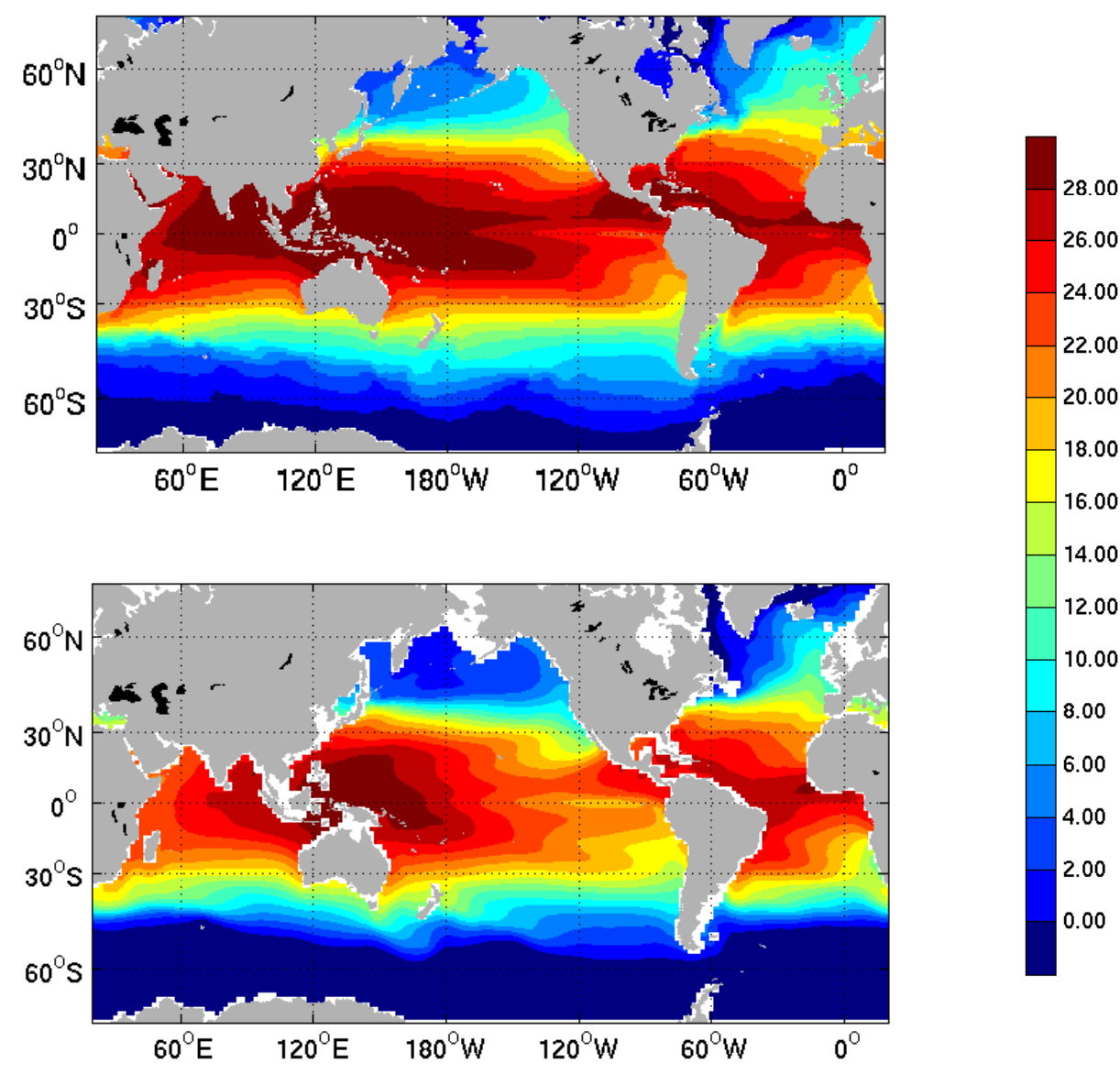

Figure 3.7: Temperature at $5 \mathrm{~m}$ averaged over years 1992-2011 in the ECCO v4 solution (top) and over years 4800-4900 of the spin-up simulation under CCSM4 LGM atmospheric conditions (bottom). 

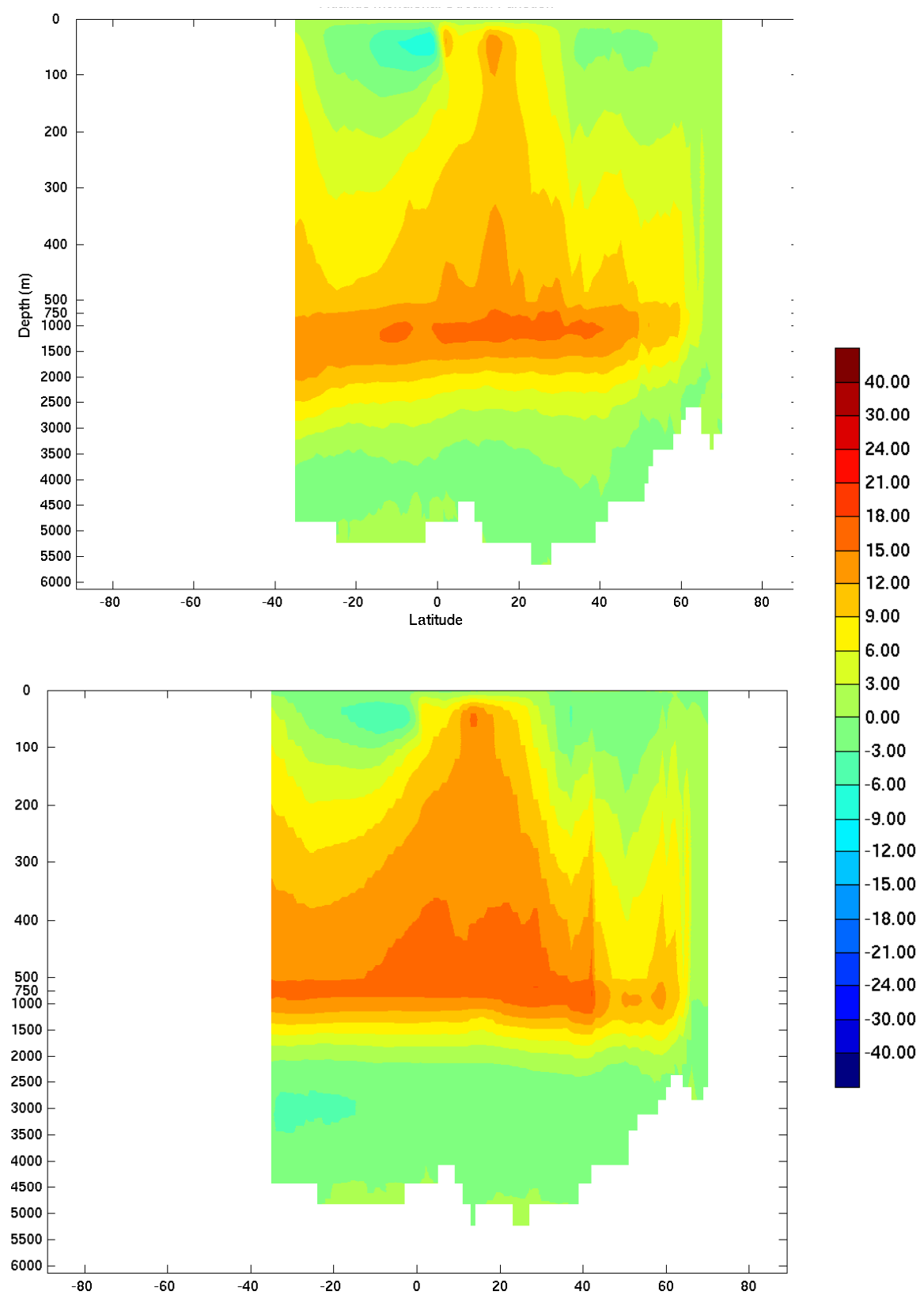

Figure 3.8: Zonal mean Atlantic meridional streamfunction plotted as a function of latitude and depth in units of Sverdrups in the ECCO v4 solution averaged over years 1992-2011 (top) and in the spin-up simulation under CCSM4 LGM atmospheric conditions averaged over years 4800-4900 (bottom). 


\subsubsection{Fitting the model to data}

Misfits between first guess NSSTs and the MARGO data set are both regional and basin-wide in scale and exceed published uncertainties in many instances (Figure 3.9). In the tropical Indian Ocean and the Southern Ocean, model temperatures are consistently higher than reported by the data. Warm model biases in the Indian and Atlantic sectors of the Southern Ocean suggest that the model Polar Front lies too far south. In the Pacific Ocean, the model east-west gradient in temperature is stronger than that seen in the data; in the North Atlantic and Arctic, the model is too warm in the east and too cold in the west. These patterns of model-data disagreement are similar to those observed in other LGM simulations [Waelbroeck et al., 2009, Braconnot et al., 2007]. As shown by histograms of misfits (Figure 3.11), typical normalized model-data misfits are larger than 1 (which would indicate consistency of the model with the data and their uncertainties) for annual, JAS, and JFM data. All three time periods show a cold bias in the model.

Spatial patterns of model-data misfit in $\overline{\mathbf{x}}_{M A R G O}$ are broadly similar to initial misfits, but misfit magnitudes have been reduced in most regions (Figures 3.10 and 3.11). Gradients of remaining misfits within some interpolated MARGO $5^{\circ} \times 5^{\circ}$ grid boxes, particularly in frontal regions where horizontal NSST gradients are large (notably, off the coast of modernday Nova Scotia), suggest that the coarseness of that gridded data product may limit how much the model-data misfit may be reduced. Several regions where costs were increased by the inverse procedure lie in the Northeast Atlantic, where the minority of observations suggesting cooler temperatures that in the first guess state were overwhelmed by nearby data suggesting warmer temperatures. The presence of coherent patterns in the model-data misfit in the solution may indicate persistent regional biases in the model or data, though more iterations could eliminate misfit covariance.

Histograms of misfits indicate improvements between iterations 0 and 9 in all seasons. 

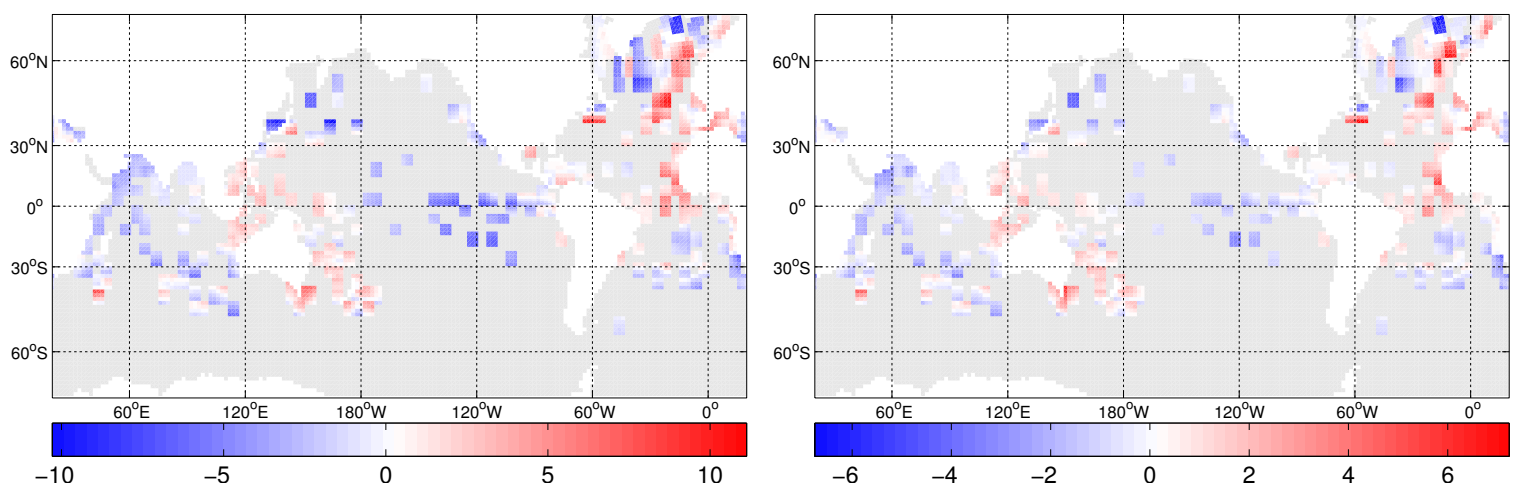

(a) Annual misfits
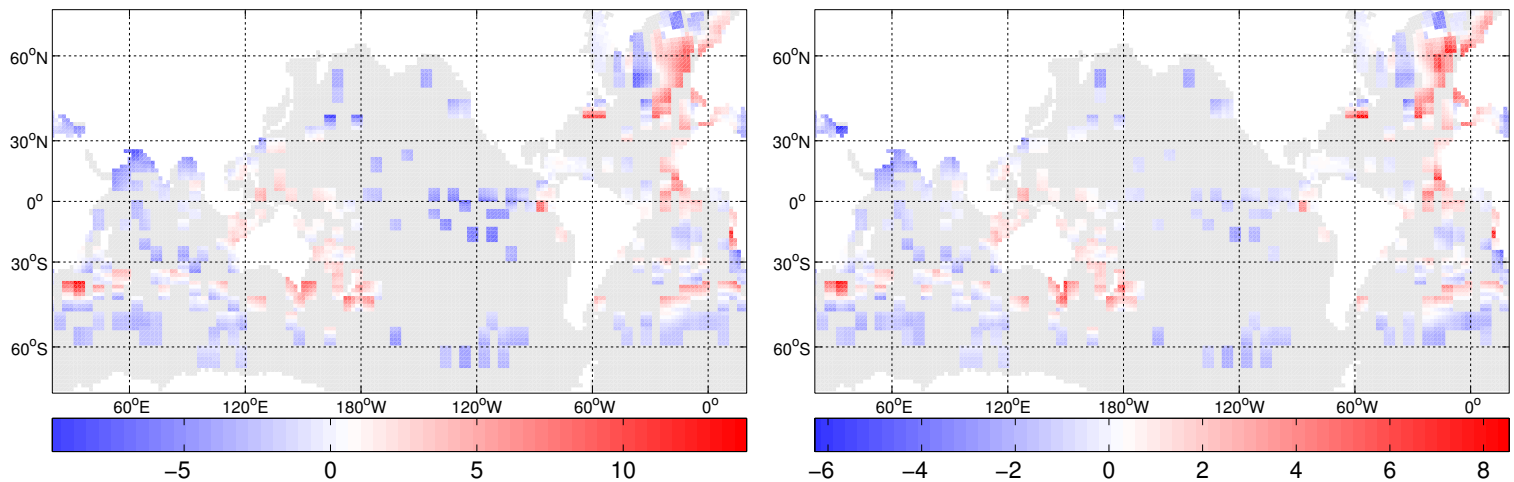

(b) January-February-March misfits
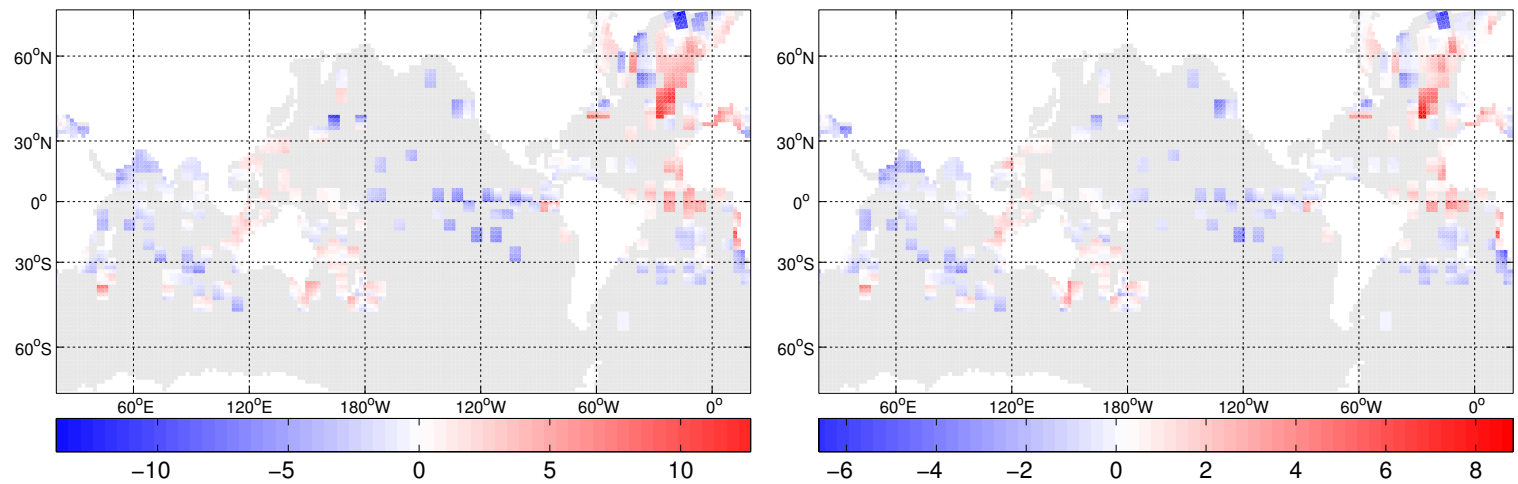

(c) July-August-September misfits

Figure 3.9: Unscaled (left, ${ }^{\circ} \mathrm{K}$ ) and scaled (right, normalized units) model-data disagreement in the initial simulation of the MITgcm. Blue (red) values indicate that the top 3 model grid boxes are cold (warm) relative to the data. Scaled misfits are computed by dividing the unscaled misfit by the data uncertainty (Figure 3.6. 

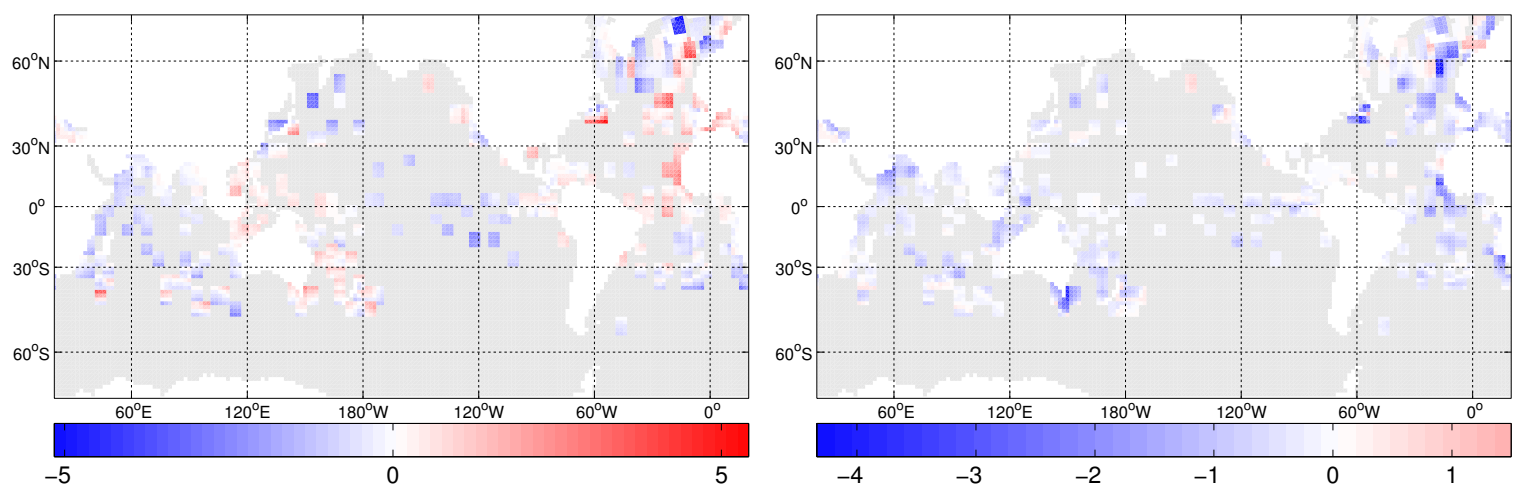

Figure 3.10: Annual model-data misfit scaled by data uncertainties after 10 iterations of the adjoint (left); as before, blue (red) values indicate that the top 3 model grid boxes are cold (warm) relative to the data. The right panel shows scaled changes in absolute misfit since iteration 0 (right); blue (red) areas indicate regions of cost reduction (increase). Results for JFM and JAS misfits are qualitatively similar.

Misfits for JFM data at iterations 9 and 10 remain unacceptably large given their estimated uncertainties, possibly due to the presence of Southern Ocean diatom records, which contribute to the disagreement for that season. Changes to misfits between iterations 9 and 10 are uniformly modest, consistent with a cost reduction that is most efficient during early iterations.

Changes in atmospheric forcings derived to fit the data are shown in Figure 3.12. Magnitudes of changes reflect prior uncertainties (listed in Table 3.12); spatial distributions of control changes. Changes in two-meter air temperature and shortwave and longwave radiation, which serve to warm or cool underlying grid boxes directly, are nearly identical and have the opposite sign of the misfits in Figure 3.9. Humidity changes are similar to temperature changes, probably because increasing humidity has a similar effect as increasing temperature by reducing latent heat loss through evaporation. Changes in precipitation are large-scale and not obviously directly related to NSST changes; because deep ocean circulation rates in the MITgcm are sensitive to surface salinity [Pillar et al., 2016], it may be that inferred precipitation changes serve primarily to reduce the model drift. Wind stress 

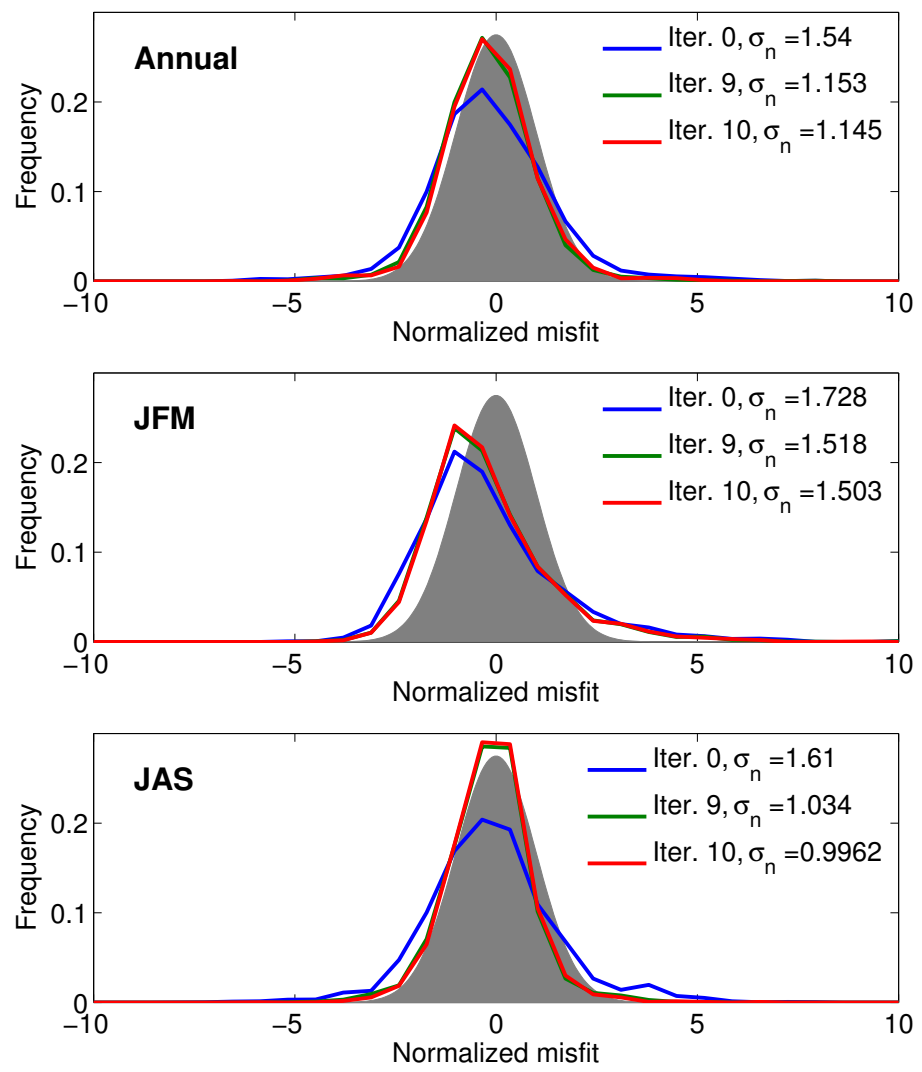

Figure 3.11: Histograms of normalized misfits to annual and boreal and austral winter average MARGO data of the $\overline{\mathbf{x}}_{M A R G O}$ solution in iterations 0,9 , and 10 . Values for iteration 9 and 10 lie very close together, and in many cases only iteration 10 (red lines) is visible. Ideally, the distribution of normalized model-data misfits would be Gaussian with zero mean and standard deviation $\sigma_{n}=1$, indicated by the grey curves. 
changes are strongest where there are MARGO data, and may change temperature locally by changing wind-stress curls and thereby local upwelling and downwelling. Visual inspection suggests that adjusted forcings have few similar spatial structures as the CCSM4 LGM-PI anomalies for most variables and thus cannot readily be interpreted as strengthening or removing those anomalies from the atmospheric controls. An exception is in surface air temperature adjustments, which run counter to CCSM4 anomalies in the North Pacific, North Atlantic, and Southern Oceans.

Changes to turbulent transports served mostly to relax the ECCO adjusted parameters back to constant background values $\left(10^{3} \mathrm{~m}^{2} \mathrm{~s}^{-1}\right.$ for isopycnal mixing and bolus transport and $10^{-4} \mathrm{~m}^{2} \mathrm{~s}^{-1}$ for diapycnal diffusivity) and are not plotted here. These changes are to be expected in the absence of deep ocean observations (from shipboard hydrography and ARGO floats) that were better fit by changes to those parameters in the ECCO v4 inversion [Forget et al. 2015b]. Changes to initial temperature and salinity are large-scale with small amplitudes (order 1/100 of the prior uncertainty) at shallow depths; in the abyss the changes are on the order of prior uncertainties with mostly-small scale variations in space. 


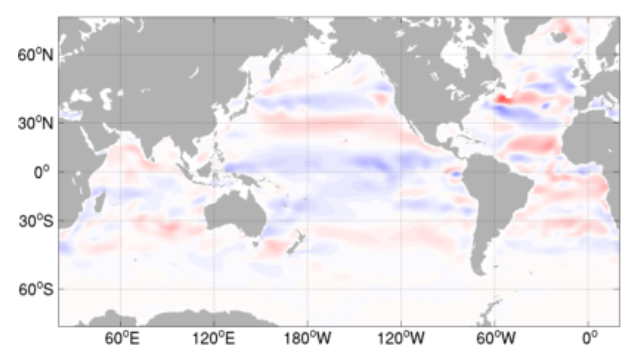

(a) Zonal wind stress anomaly

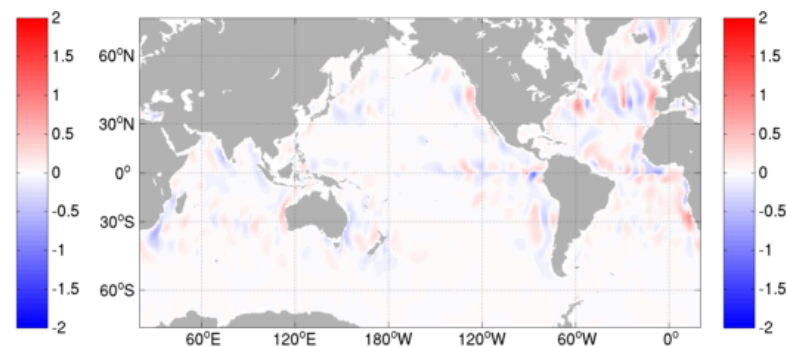

(b) Meridional wind stress anomaly

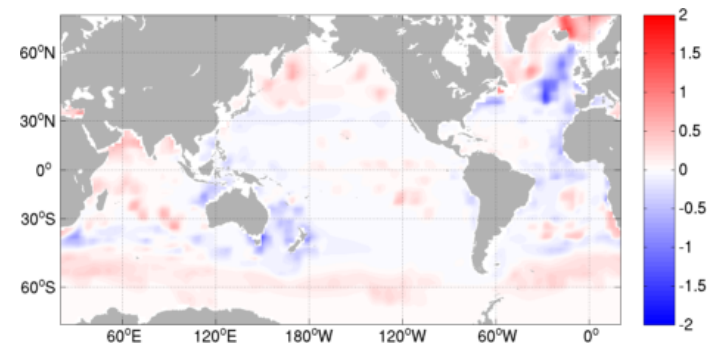

(c) Air temperature at $2 \mathrm{~m}$ anomaly

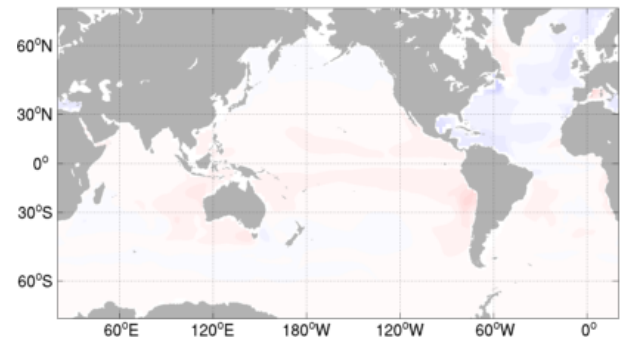

(d) Precipitation anomaly

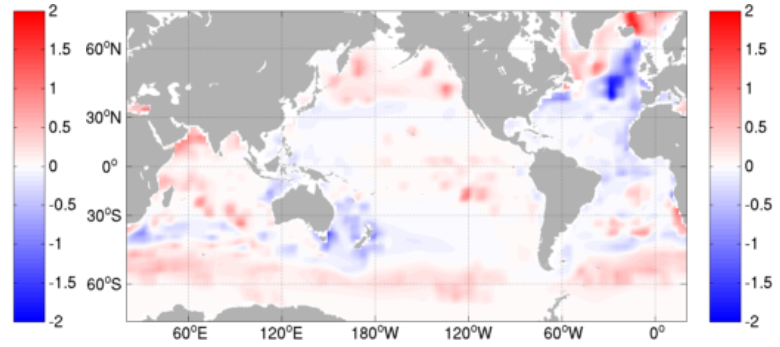

(e) Specific humidity anomaly

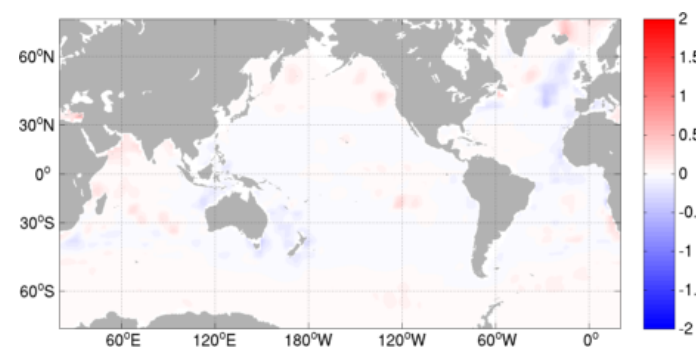

(f) Longwave radiation anomaly

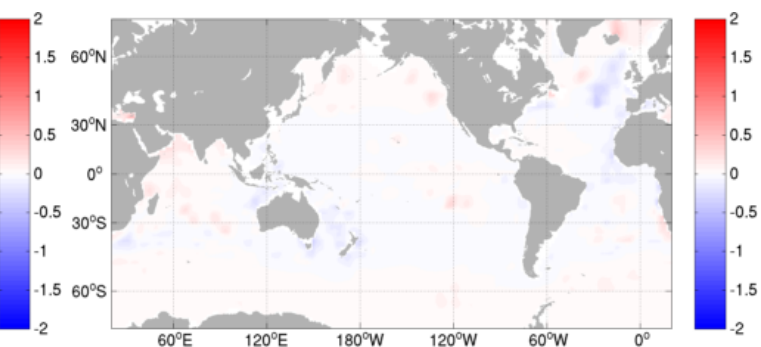

(g) Shortwave radiation anomaly

Figure 3.12: Annual mean control changes used to generate $\overline{\mathbf{x}}_{M A R G O}$, scaled by their prior uncertainties (units of $\sigma$; see Table 3.1). 


\subsubsection{Analysis of the state estimate}

This section describes several features of interest in the LGM state estimate. Quantities discussed were chosen to facilitate comparison with previously published results, and the focus is on the Atlantic, where there is a higher density of observations. Subsequent analyses will focus more on other regions of interest such as the Pacific and Southern Oceans. In several instances, distinctions are drawn between simulations at iterations 9 and 10 of the adjoint estimation procedure; unless otherwise noted, results refer to iteration 10.

\subsubsection{Near-sea surface temperatures}

Global distributions of LGM-PI NSST anomalies for annual, JFM, and JAS time periods are shown in Figure 3.13, These anomalies can be interpreted as an alternate interpolation to that of Waelbroeck et al. [2009] (Figure 3.1), one which satisfies the dynamical equations of the MITgcm. Differences between these interpolations are due both to differences in reconstructed LGM values and to the different modern NSST estimates used Gouretski and Koltermann [2004] vs ECCO v4). Moreover, the interpolation of Waelbroeck et al. [2009] does not explicitly account for uncertainties, and some prominent anomaly values (e.g., in the Pacific) arise from LGM reconstructed values with large uncertainties.

Reconstructed LGM-PI NSST anomalies indicate cooling nearly everywhere except for modest regional warming in the western tropical Pacific, western midlatitude North Atlantic, and boreal winter Arctic. The warming in the Arctic may be important for the maintenance of a sea ice-free summer Northeast Atlantic, which is suggested by the MARGO data and discussed further below. Regions of strongest cooling include the East and North Pacific, North Atlantic, and Southern Oceans. Pronounced east-west gradients in cooling are evident in the tropical Pacific and Southern Atlantic, though in the Pacific the gradient is of the opposite sign of MARGO. Cooling of more than 5 degrees in the Northeast Atlantic is evident 
in all seasons, but the the east-west gradient described in MARGO is only clearly evident in JAS. That the model fits the data in a quantifiable sense but does not reproduce some features in the MARGO interpolation argues against the robustness of those features. Reconstructed NSST anomalies differ from those found by DW14 in the subtropical and tropical Atlantic, where those authors inferred modest warming at the LGM; however, as they noted, those regions are ill-constrained by data.

\subsubsection{Barotropic streamfunction}

Model LGM and LGM-PI anomaly barotropic streamfunctions are shown in Figure 3.14. Barotropic transports are somewhat larger practically everywhere, absent small-scale changes near western boundaries (e.g., adjacent to the model Gulf Stream and Kuroshio) indicating shifts in the mean positions of those currents. More vigorous large-scale gyre circulations are consistent with a windier LGM. Some features of the picture are similar to results of DW14, who found increased southward return flow in the subtropical Atlantic gyre and a more zonal Gulf Stream, though the strong eastward shift of the Atlantic subpolar gyre that they describe is not evident.

\subsubsection{AMOC and meridional heat transport}

Iteration 10 zonally averaged Atlantic meridional overturning strength (Figure 3.15, cf. 3.2) indicates an upper cell that is shallower in the tropics and uniformly stronger than the ECCO v4 estimate by 5-10 Sverdrups. This picture is qualitatively consistent with the CCSM4 LGM simulation and somewhat stronger than the first-guess state (Figure 3.8). However, the iteration 9 streamfunction is substantially different, indicating AMOC strengths $\sim 10$ Sv less than in iteration 10. Larger values are likely symptomatic at least in part of higher surface salinities in the high-latitude Atlantic in iteration 10 due to precipitation adjustments. It is possible that these salinity changes, possibly compensated by other changes in the control 

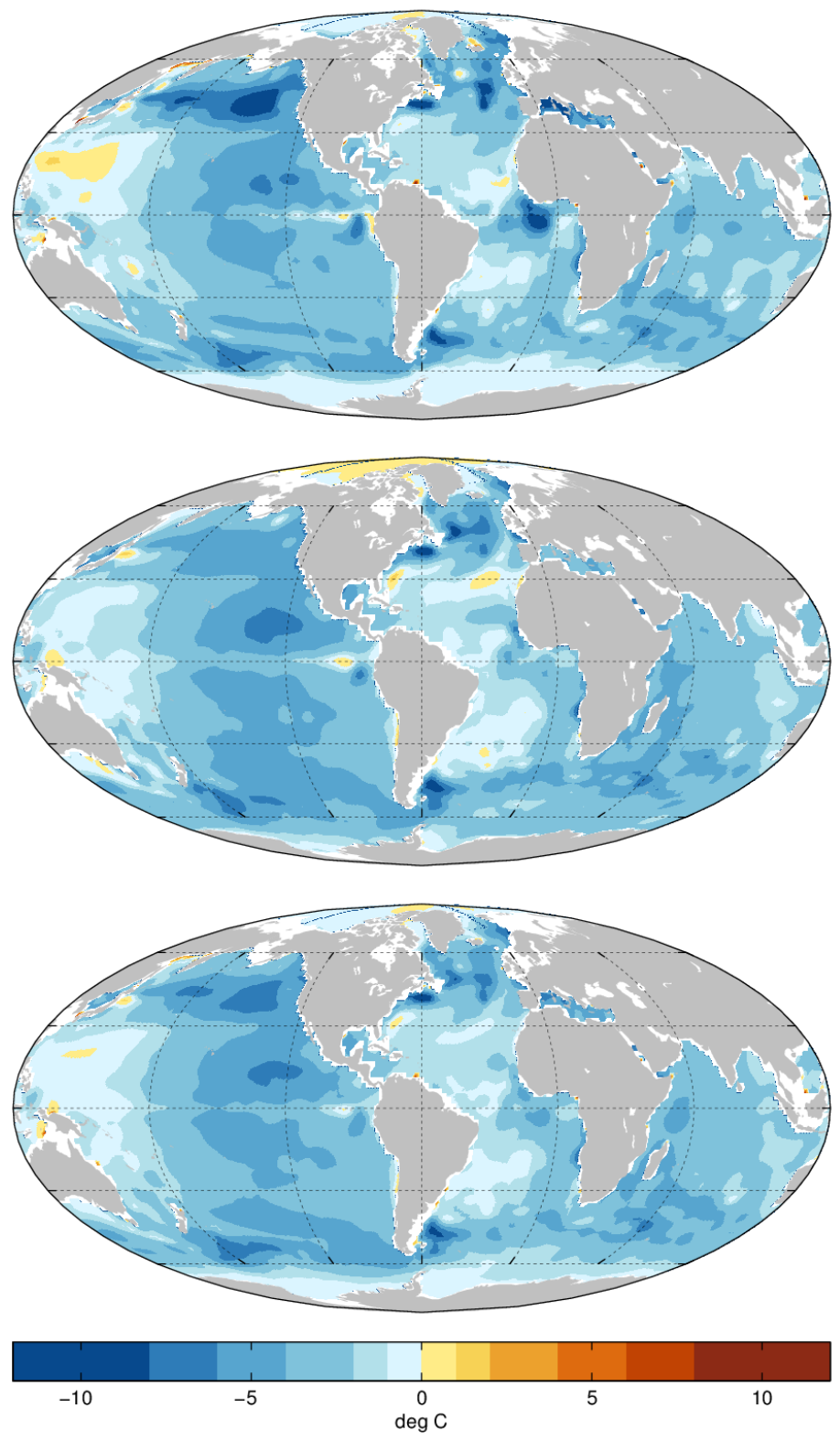

Figure 3.13: LGM-modern NSST temperature anomalies averaged over the top three model grid boxes (spanning 30 meters) computed as the difference between $\overline{\mathbf{x}}_{M A R G O}$ and ECCO v4 for means over July-August-September (top), January-February-March (middle), and the annual mean (bottom). LGM temperatures are computed over the last ten years of the 100year $\overline{\mathbf{x}}_{M A R G O}$ simulation to avoid initial transience as the model adjusts to control changes in initial temperature and salinity. 

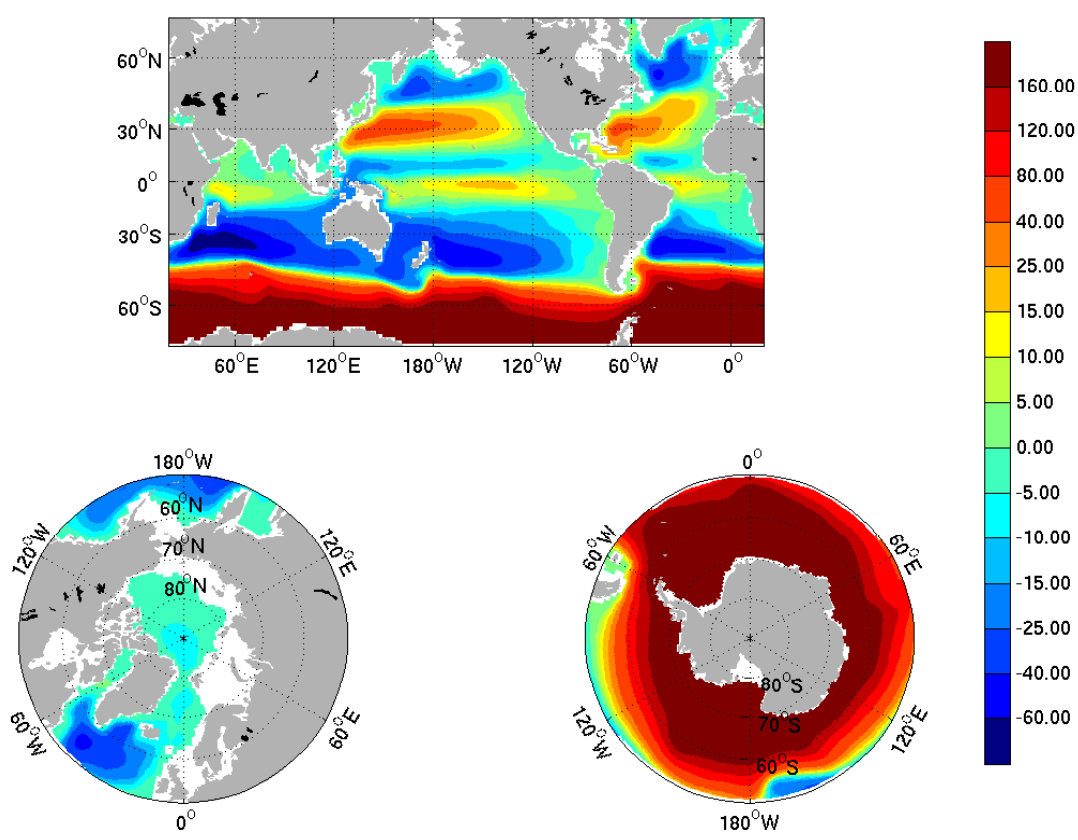

0.00

$-5.00$

$-10.00$

$-15.00$

$-25.00$

$-40.00$

$-60.00$
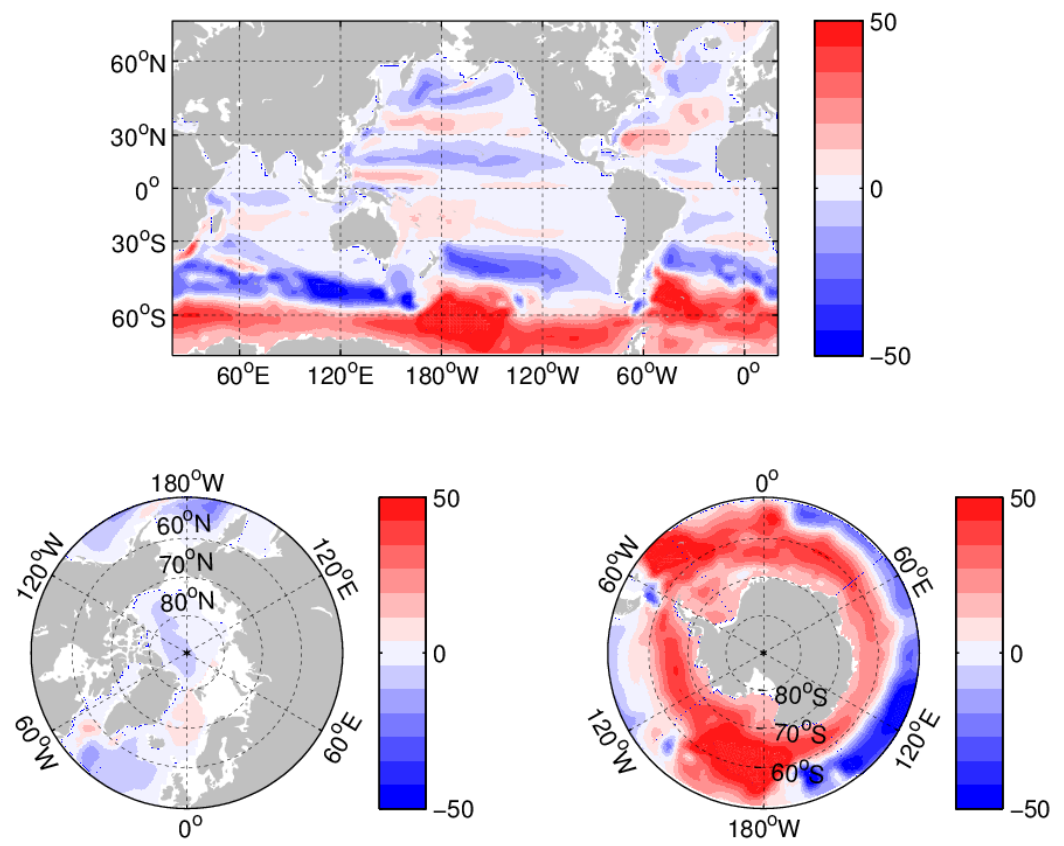

Figure 3.14: LGM (top) and LGM-modern barotropic streamfunctions plotted in Sverdrups. The modern streamfunction is computed from the ECCO v4 solution averaged over 19922011. 
vector, had a modest effect on the model-data misfit, but changed abyssal circulation substantially. Time series of drifts in AMOC strength at 25, 35, 45, and $55 \mathrm{deg} \mathrm{N}$ (not shown) are small at 100 years (less than $.5 \mathrm{~Sv}$ per decade) after transient intervals of several decades at the beginnings of simulations, suggesting that model spin-up is not the problem, as was suggested by Zhang et al. [2013] to explain differences between PMIP2 simulations. AMOC structure and strength also vary in the preceding iterations. That the model-data misfit in iterations 9 and 10 is similar but corresponding AMOC strengths are different suggests that the MARGO dataset is ill-suited to constraining AMOC strength, echoing results of KurahashiNakamura et al. [2013].

The ocean plays an important role in the climate system by transporting heat northward in the Atlantic Ocean; many studies invoke changes in heat transport to explain regional patterns of warming or cooling at the LGM and during other climate intervals. At iteration 9, heat transport in the Northern Hemisphere is slightly reduced $(\sim 10 \%)$ from the modern ECCO estimate, while transports in the Southern Hemisphere are slightly increased, commensurate with a modestly weakened upper branch and modestly strengthened lower branch of the AMOC (Figure 3.16). By contrast, iteration 10 shows the opposite pattern, with stronger poleward transport in the north and weaker in the south relative to the modern. As with AMOC strength, given the relative insensitivity of the model-data misfit to these qualitative differences, a conclusion is that the MARGO data and the model used lack the power to constrain changes in ocean heat transports.

\subsubsection{Northern Hemisphere sea ice}

The $\overline{\mathbf{x}}_{M A R G O}$ Arctic Ocean is filled with sea ice year-round, and some perennial sea ice extends down the western coasts of Canada and Greenland (Figure 3.17). In winter, sea ice extends south of the Newfoundland coast and covers parts of the Nordic seas. The reconstruction agrees with an estimate by De Vernal et al. [2006], who analyzed planktonic assem- 


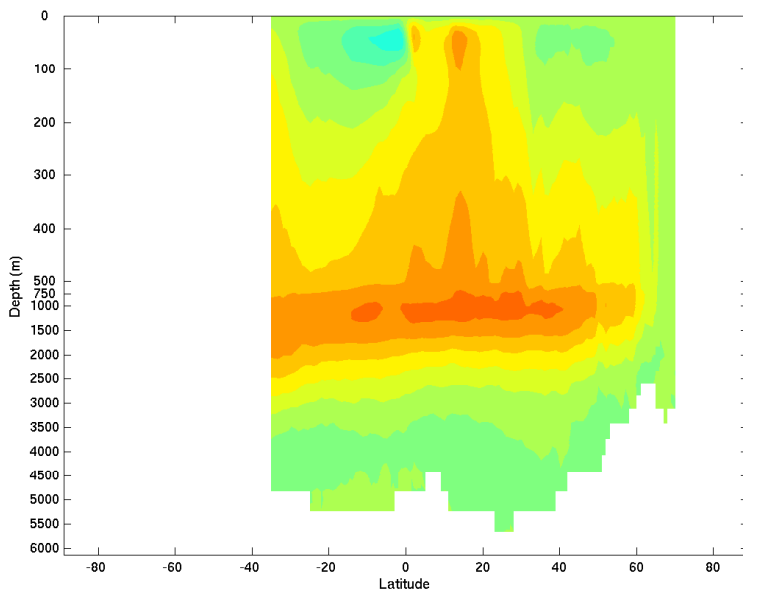

\subsection{0 \\ 30.00 \\ 24.00 \\ 21.00 \\ 18.00 \\ 15.00 \\ 12.00}

9.00

$-6.00$
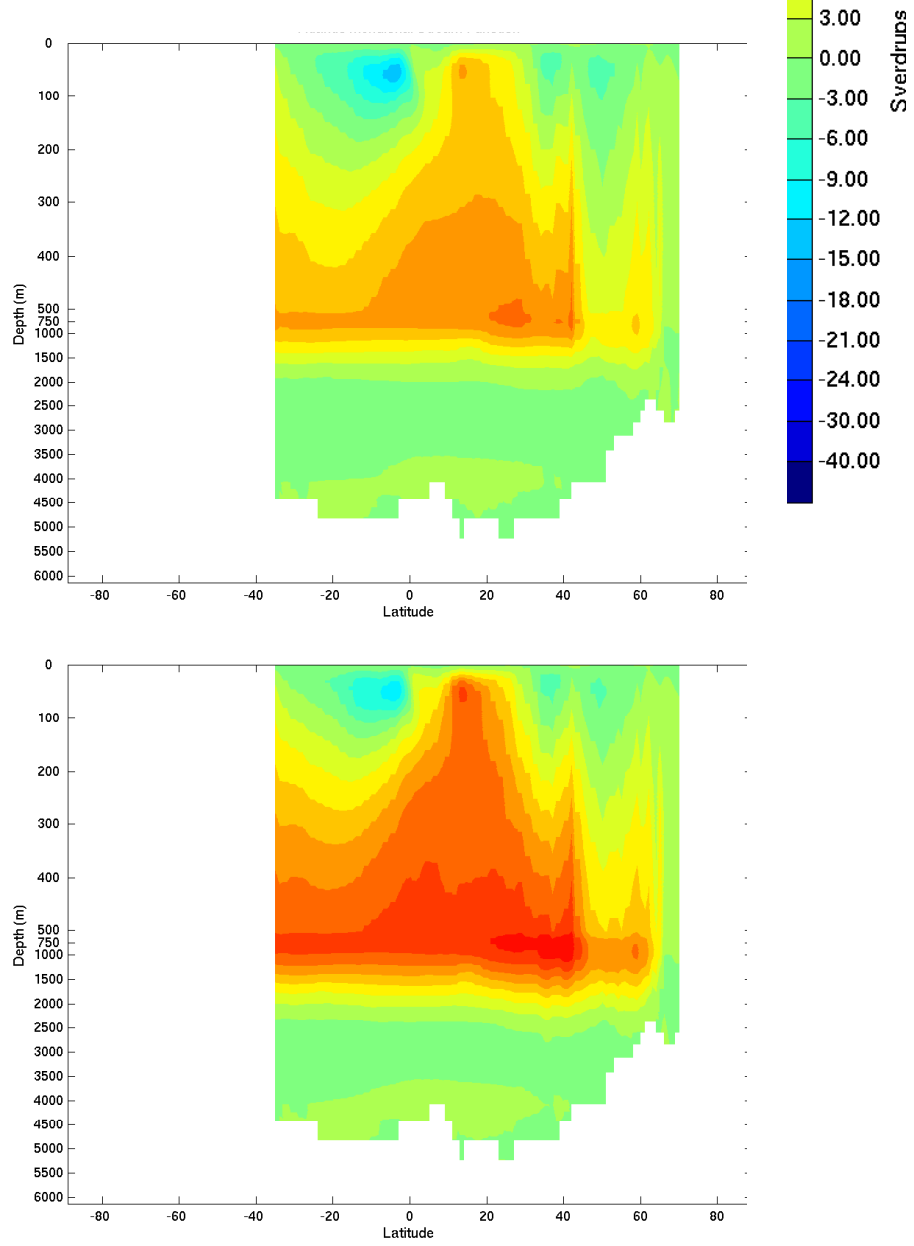

Figure 3.15: Atlantic zonal mean streamfunctions in ECCO v4 (top, 1992-2011 mean), $\overline{\mathbf{x}}_{M A R G O}$ after 9 iterations (middle) and $\overline{\mathbf{x}}_{M A R G O}$ at the final (10th) iteration (bottom). Large changes in AMOC strength between the 9th and 10th iterations, when model-data misfit is similar, suggest that the data do not constrain this quantity well. 

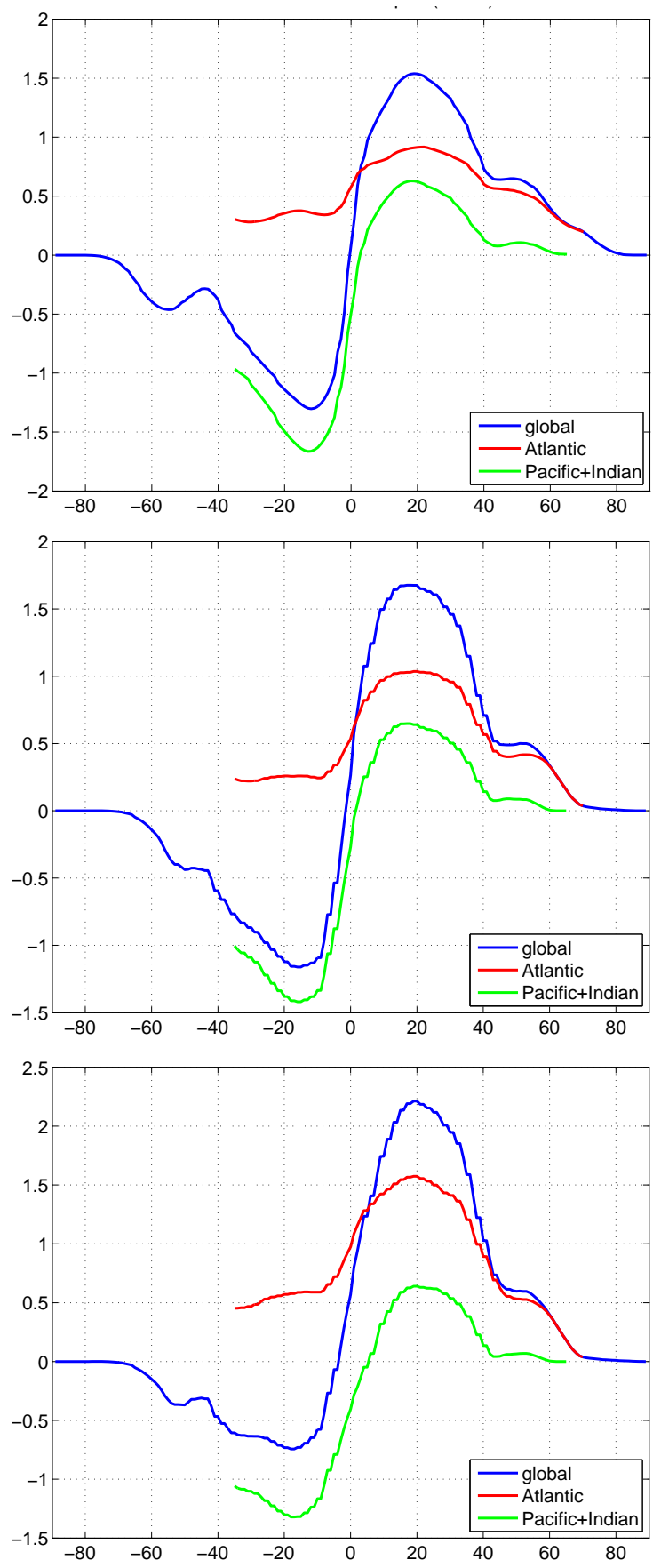

Figure 3.16: Heat transport in PW as a function of latitude for ECCO v4 (1992-2011 mean, top) and $\overline{\mathbf{x}}_{\text {MARGO }}$ iterations 9 (middle) and 10 (bottom). Note the different vertical axes. 
blages and dinoflagellate cysts in the MARGO database and proposed seasonal and perennial sea ice extents (Figure 3.18). Their reconstruction for winter sea ice from foraminifera more closely resembles $\overline{\mathbf{x}}_{\text {MARGO }}$ than does the reconstruction from dinocysts, possibly because dinocyst measurements were downweighted in the MARGO gridded product due to their high uncertainties. Reconstructed LGM sea ice is substantially greater (in extent and total volume) than observed in the modern ocean and simulated in ECCO v4; for instance, modern sea ice does not cover the entire Arctic year-round. Additional experiments are necessary to test to what extent these changes are due to colder temperatures and other atmospheric forcings versus other changes, such as closing the Bering Strait and shrinking the areal extent of the Arctic basin.

Winter ice thicknesses in the central Arctic are roughly 10 meters, with an abrupt change in thickness evident just north of Greenland (not shown), likely driven by warmer air temperatures (Figure 3.19) to fit MARGO observations of warm summer temperatures there. The reader is reminded that snow and ice albedos were reduced in the model to avoid monotonic sea ice growth, and as such any reconstructed features in the sea ice should be viewed cautiously. More sophisticated models (ideally able to simulate thick sea ice as has been hypothesized for the LGM Arctic) and more observations are necessary to better constrain these properties.

\subsubsection{Mixed layer depth}

Differences between the spatial distributions of NH winter mixed layer depth in $\overline{\mathbf{x}}_{M A R G O}$ and the modern ocean (Figure 3.20) are visible in both iterations 9 and 10, suggesting that they may be a robust feature of the reconstruction. Large MLD changes off of Nova Scotia in the glacial state estimate compared to the modern are likely an artifact of penalizing model deviations over a $5^{\circ} \times 5^{\circ}$ grid box in a region with a large background SST gradient, leading to large wind stresses in that region that change NSSTs through local upwelling. MLD in 

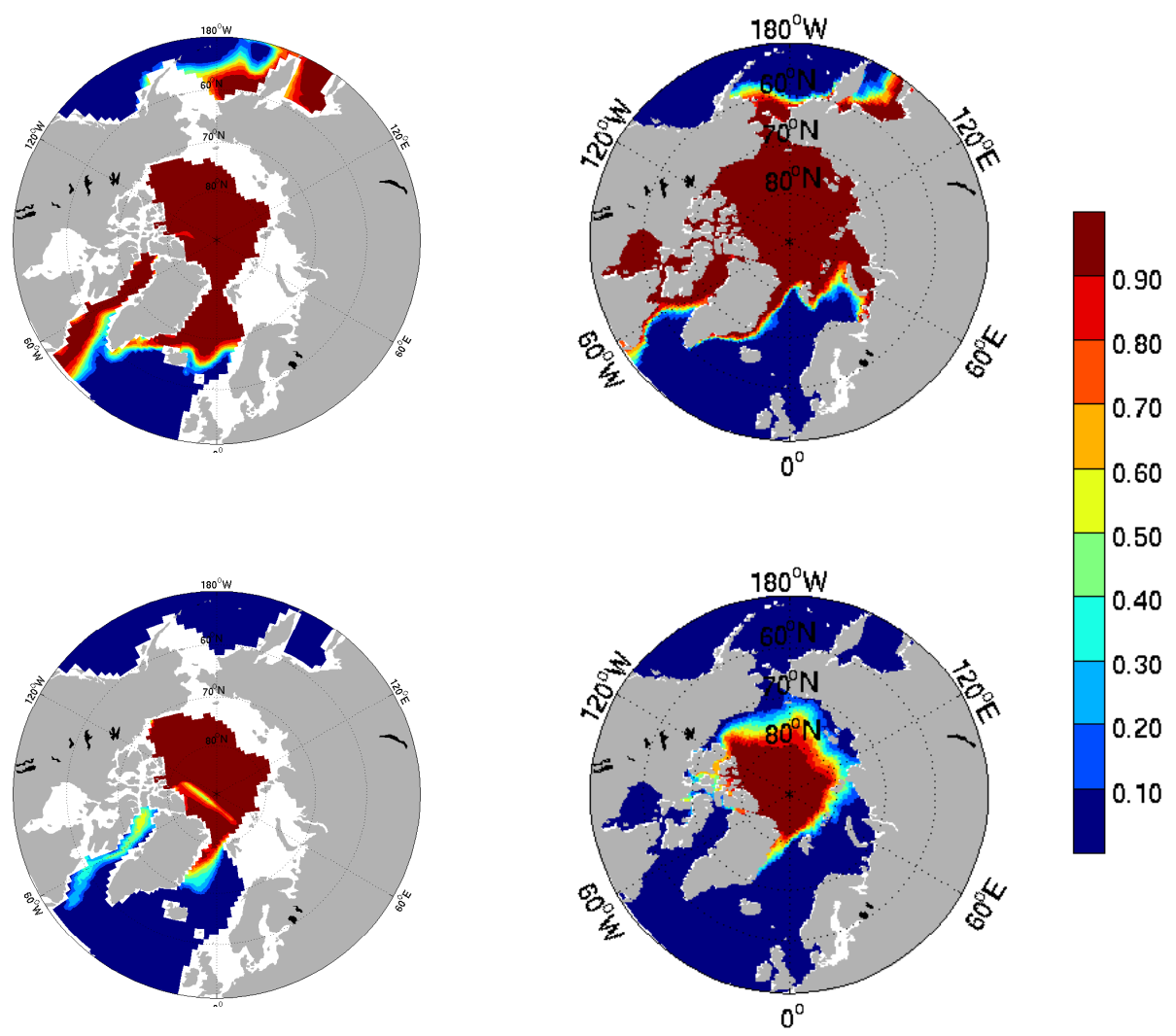

Figure 3.17: Sea ice concentration in $\overline{\mathbf{x}}_{M A R G O}$ (left) and from modern observations [Comiso, 1999] in March (top) and September (bottom).

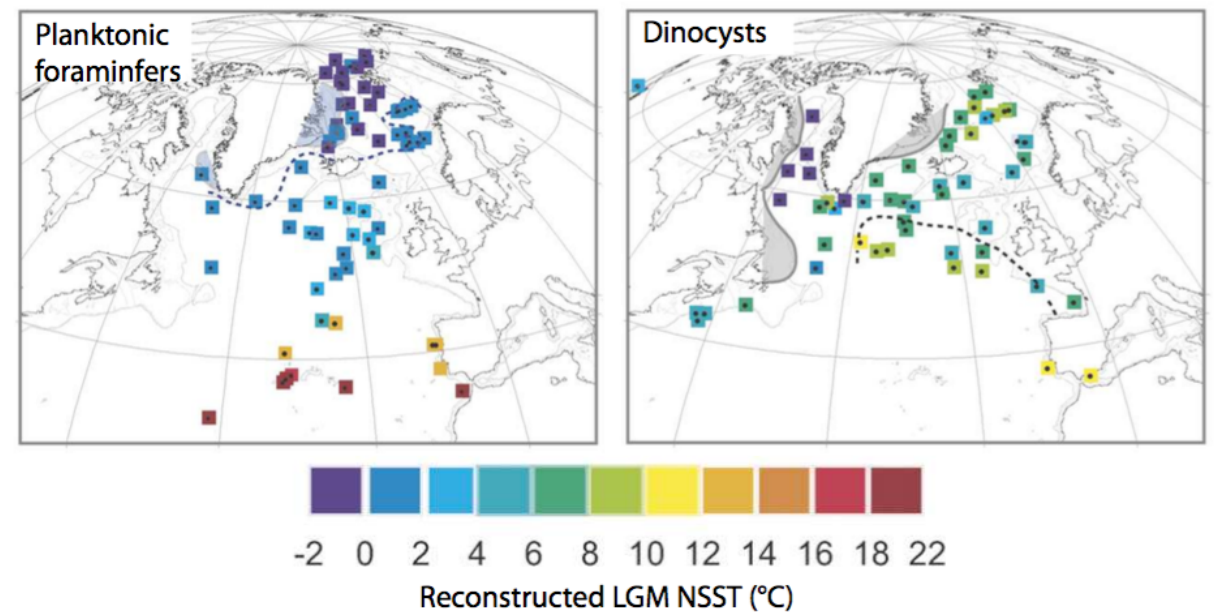

Figure 3.18: Modified figure from De Vernal et al. [2006] used in Dail [2012b] showing MARGO LGM NSST reconstructions from planktonic foraminiferal assemblages (left) and from dinoflagellate cysts (dynocysts, right) and estimates of LGM sea ice extent based on these data. In both plots, grey shading shows estimates of perennial sea ice, and the dotted lines illustrate estimated maximum winter sea ice extent. 


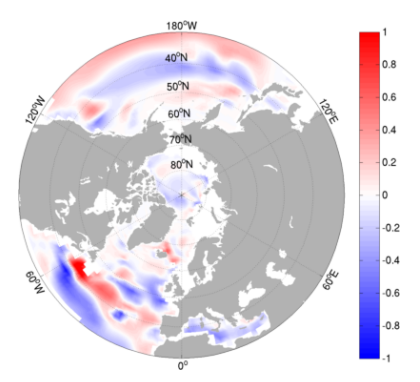

(a) Zonal wind stress anomaly $(\mathrm{Pa})$

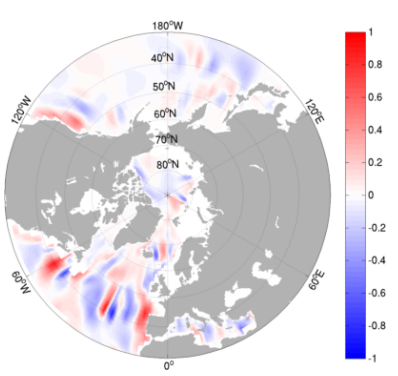

(b) Meridional wind stress anomaly $(\mathrm{Pa})$

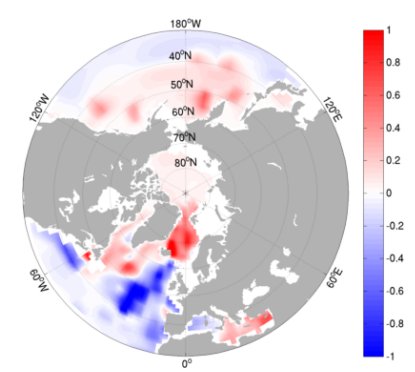

(c) Air temperature at $2 \mathrm{~m}$ anomaly $\left({ }^{\circ} \mathrm{K}\right)$

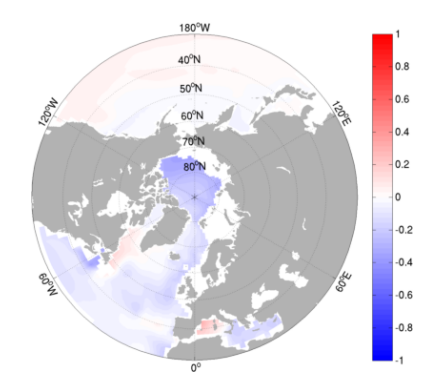

(d) Precipitation anomaly $\left(\mathrm{m} \mathrm{s}^{-1}\right)$

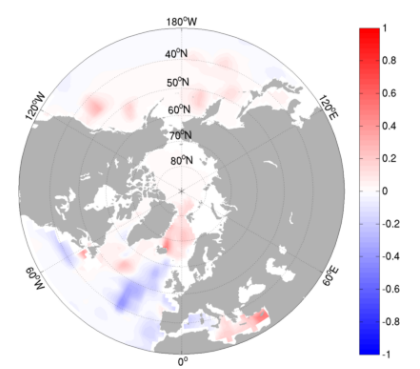

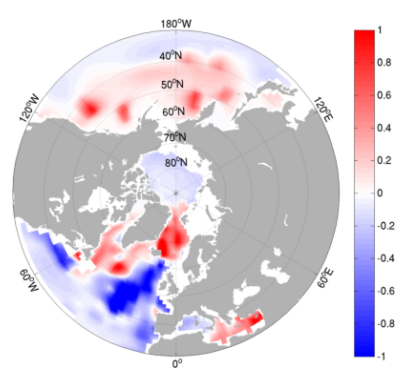

(e) Specific humidity anomaly (unitless)

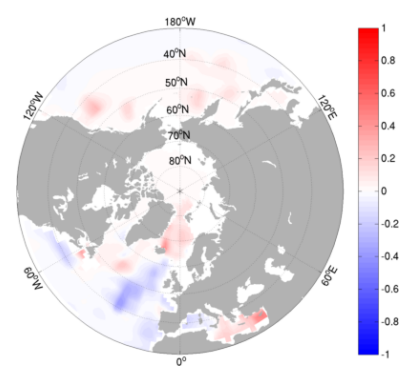

(f) Longwave radiation anomaly $\left(\mathrm{W} \mathrm{m}^{-2}\right)(\mathrm{g})$ Shortwave radiation anomaly $\left(\mathrm{W} \mathrm{m}^{-2}\right)$

Figure 3.19: Annual mean control changes used to generate $\overline{\mathbf{x}}_{M A R G O}$ in the Northern Hemisphere, scaled by their prior standard deviation (Table 3.1). 
the Labrador Sea may be reduced due to the greater sea ice extent in that region, which can enhance upper ocean stratification. Given that horizontal gradients of geochemical tracers such as $\delta^{18} \mathrm{O}_{c}$ in the surface ocean can be large relative to vertical gradients in the deep ocean, horizontal changes in the contributions of ventilating regions to deep water masses can change those abyssal tracer quantities substantially, as described by Miller et al. [2012] for temperature and salinity in the Southern Ocean. One can speculate that changes in MLD are a circulation change that could explain a substantial part of the changes in abyssal tracers during the LGM and subsequent deglaciation; to what extent such changes would be accompanied by changes in abyssal density field and hence large-scale circulation requires further investigation.

\subsubsection{Evaluating the fit of $\overline{\mathbf{x}}_{M A R G O}$ to LGM $\delta^{18}$ O observations}

Motivated by the apparent inability of the MARGO data to constrain features of the abyssal circulation, we next evaluate the utility of oxygen isotope measurements, which have been hypothesized to constrain paleo circulation both in steady [e.g., Lund et al., 2011] and transient intervals (Chapter 2 and references therein). The approach is to integrate the MITgcm forward using the $\overline{\mathbf{x}}_{M A R G O}$ circulation under "upstream" LGM mixed layer (ML) boundary conditions based on planktonic foraminiferal records and compare simulated deep-sea $\delta^{18} \mathrm{O}_{c}$ to "downstream" benthic foraminiferal observations. If benthic data and model abyssal $\delta^{18} \mathrm{O}_{c}$ are in agreement, then the model agrees with the data and there is little to be gained by including $\delta^{18} \mathrm{O}$ in the cost function. We also assess whether $\delta^{18} \mathrm{O}_{c}$ model-data misfits differ

in the two abyssal circulation scenarios in iterations 9 and 10 . Sensitivity of $\delta^{18} \mathrm{O}_{c}$ misfits to those scenarios could indicate that including those data can refine inferences of AMOC strength and meridional heat transport.

Planktonic $\delta^{18} \mathrm{O}$ observations are compiled from Caley et al. [2014] and Oliver et al. [2010] and come from multiple species, though most are G. bulloides and N. pachyderma 

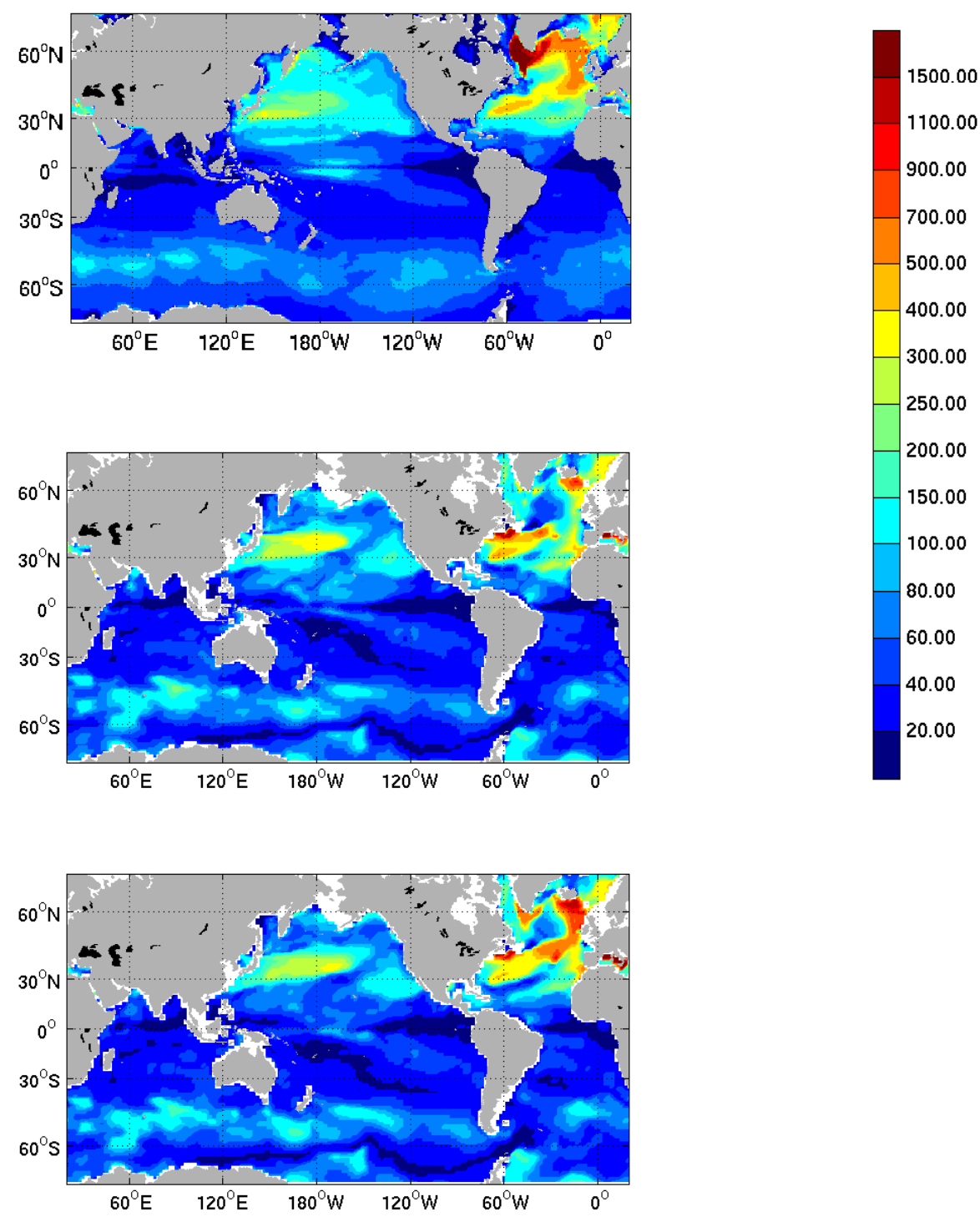

Figure 3.20: Mean March mixed layer depth in meters computed via Kara et al. [2000] in ECCO v4 (top) and $\overline{\mathbf{x}}_{\text {MARGO }}$ in iteration 9 (middle) and 10 (bottom). 
$s$. The distribution of observations is global, but with relatively many observations in the tropics and North Atlantic and many fewer observations in the Pacific and south of $60^{\circ} \mathrm{S}$. Benthic observations are compiled from Oliver et al. [2010] and include the Marchal and Curry [2008] data. Benthic data show smaller horizontal gradients than planktonic observations because the deep ocean is primarily filled by polar regions in both hemispheres, which have similarly high $\delta^{18} \mathrm{O}_{c}$ values. Core sites are distributed inhomogeneously in space, with many observations from the mid-Atlantic ridge and few observations from greater than 4000 $\mathrm{m}$ and in the Pacific. Genera are primarily but not exclusively Cibicidoides.

The distribution of planktonic observations is insufficient alone to describe ML values in each roughly $2 \times 2$ model grid box, so some form of interpolation is necessary to derive a $\mathrm{ML} \delta^{18} \mathrm{O}_{c}$ boundary condition. One procedure would be to derive spatial covariances of $\delta^{18} \mathrm{O}$ from modern distributions or LGM simulations and use the covariances in an objective mapping procedure. Here, a simpler procedure is found to fit planktonic data adequately and incorporate salient spatial covariance information. NSSTs from $\overline{\mathbf{x}}_{M A R G O}$ were combined with the seawater oxygen isotope distributions of LeGrande and Schmidt [2006] (with a correction of $1 \%$ corresponding to a deglacial decrease in $\delta^{18} \mathrm{O}$ of benthic seawater [Adkins et al., 2002], but note that this value may be poorly constrained [Miller et al., 2015, Wunsch, 2016]). These fields were combined using the empirical formula of Bemis et al. [1998],

$$
\delta^{18} \mathrm{O}_{c}=3.4+\delta^{18} \mathrm{O}_{s w}-0.21 T
$$

where $\delta^{18} \mathrm{O}_{s w}$ is the $\delta^{18} \mathrm{O}$ of seawater standardized by VSMOW and $T$ is temperature in degrees Celsius.

Two such fields were derived in this way. Boundary conditions used to simulate deep $\delta^{18} \mathrm{O}_{c}$ were derived using temperatures in the coldest month within each grid box, as deep waters are typically formed in the coldest month of the year, when overlying waters are dens- 
est and most prone to vertical instability. A second field was constructed using NSST values computed from the annual mean of the state estimate, which better represents temperatures at which planktonic foraminifera calcify their shells; these values are compared to planktonic observations in Figure 3.22. The extent to which the annual mean NSST adequately represents temperatures in the growing season of planktonic foraminifera depends on several factors including the foraminiferal species. The annual average is a reasonable time period for G. bulloides (D. Oppo, personal communication), which make up most of the data, and was also used in the model-data comparison of Caley et al. [2014]; the standard deviation of the monthly values is used to quantify uncertainty in this approach in Figure 3.22. Annual mean $\mathrm{ML} \delta^{18} \mathrm{O}_{c}$ conditions agree with planktonic data to within the model annual cycle at many locations, with disagreements in midlatitudes near large meridional temperature gradi-

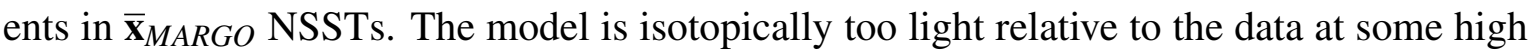
latitude locations. A truly quantitative comparison of derived annual-average $\delta^{18} \mathrm{O}_{c}$ boundary conditions and data would require estimating uncertainties of planktonic data to represent $\delta^{18} \mathrm{O}_{c}$ in surface model grid boxes, which is challenging given the diverse sources of error and which is not attempted here. Instead, we simply note that the relationship appears overall to be satisfactory and proceed. Eventually it may be desirable to have ML $\delta^{18} \mathrm{O}_{c}$ conditions in the control vector.

The MITgcm LGM configuration was integrated forward separately under controls derived in iterations 9 and 10 for 1470 years using vertically nonuniform time steps, and equilibrated values are estimated using the extrapolation approach outlined in Chapter 4. Estimates of equilibrium values at data locations drift by at most $0.0002 \%$ per year after 1470 years, an indication that these values are close to a true equilibrium.

The distribution of simulated $\delta^{18} \mathrm{O}_{c}$ is complex, reflecting spatial variations in the boundary conditions and the model tracer transport flow. To illustrate model-data misfits we focus on the region between 10 and $20^{\circ} \mathrm{W}$, which includes a large concentration of benthic $\delta^{18} \mathrm{O}_{c}$ 
observations lying along the Mid-Atlantic Ridge. The zonal mean of modeled tracer and benthic data in this region from the 1470-year iteration 10 simulation are shown in Figure 3.23 . The distribution of the southern-source high- $\delta^{18} \mathrm{O}_{c}$ water is reminiscent of modern Antarctic Bottom Water and penetrates far into the abyssal Atlantic. A tongue resembling Antarctic Intermediate Water is also evident in the Southern Hemisphere. An isolated maximum in the northern end of the domain could be due to a deep North Atlantic contribution or some lateral transport, possibly from the Western North Atlantic. $\delta^{18} \mathrm{O}_{c}$ values of Northern- and Southern- source end members diagnosed from this figure are roughly 3.5 and $4 \%$, respectively. These values are less than the those inferred by Lund et al. [2011], who extrapolated sediment core data from the Atlantic to estimate that the southern component water had $\delta^{18} \mathrm{O}_{c}=5 \%$ and the northern component had $\delta^{18} \mathrm{O}_{c} \leq 4.0 \%$, and by Oppo et al. [2015], who estimated values of greater than $\geq 4.84 \pm 0.10 \%$ and equal to $4.48 \pm 0.07 \%$. While this disagreement could be due to biases in the model or in those studies, this comparison suggests that estimated high latitude surface values of $\delta^{18} \mathrm{O}_{c}$ may be too low, as was also suggested by the misfit to planktonic observations.

Benthic observations in the region between 10 and $20^{\circ} \mathrm{W}$ do not appear to be spatially coherent. In the relatively narrow latitude band chosen, zonal variations in tracer are unlikely to explain much of the dispersion. A detailed accounting of uncertainties in the benthic records is not undertaken here, but uncertainties are expected to be large and to vary substantially by observation, particularly as some core sites average over a large number of LGM-era observations to obtain a measurement (thereby mitigating aliasing of higher-frequency signals onto the mean), while other observations come from single time points. Instead we use $\sigma=0.2 \%$ as an estimate of uncertainty, following Chapter 2 and Marchal and Curry [2008]. While model-data misfits are large relative to this uncertainty, no coherent patterns are apparent, and it is not immediately clear how the model could be changed in order to improve the fit. 
Global normalized model-data misfits (Figure 3.24) from iterations 9 and 10 both have standard deviations of 2.2; iteration 9 misfits are slightly biased positive. That the model and data do not agree would imply that the data have new information about the LGM that can hypothetically be incorporated in the model; however, the lack of consistency in the data set suggests that the error estimate of $0.2 \%$ may be too small. If so, this results calls into question the utility of using these $\delta^{18} \mathrm{O}$ observations in the LGM state estimate. Moreover, similarities in model-data misfits for iterations 9 and 10 make it unlikely that the $\delta^{18} \mathrm{O}$ data could distinguish between the two AMOC strengths and meridional heat transports in those simulations. If, similar to experiments conducted in Marchal and Curry [2008], observational uncertainties were doubled from 0.2 to $0.4 \%$, then the normalized misfits would have standard deviations of roughly 1, suggesting an acceptable fit between the model and data. However, this result is not surprising given the estimates of the differences between Northern- and Southern-source watermass of roughly $0.5 \%$. An uncertainty of $0.4 \%$ would mean that the data probably cannot distinguish between end members, at least in the Atlantic, and could be fit to nearly any deep distribution.

\subsection{Discussion}

This chapter presents a picture of the LGM ocean that is plausible in the sense that it fits a subset of the available data within uncertainties and obeys physical constraints as represented in a coarse-resolution OGCM. The solution shown should be taken as a current best estimate of the LGM circulation, subject to revision as the model is fit to more observations and additional adjoint iterations are performed. Care should be taken in interpreting any feature of the solution as representing true LGM conditions, not least because of model biases that have not been quantified. The data used, paltry relative to that available for modern state estimation, are inadequate to identify a single, optimal LGM state estimate, and any inferred 


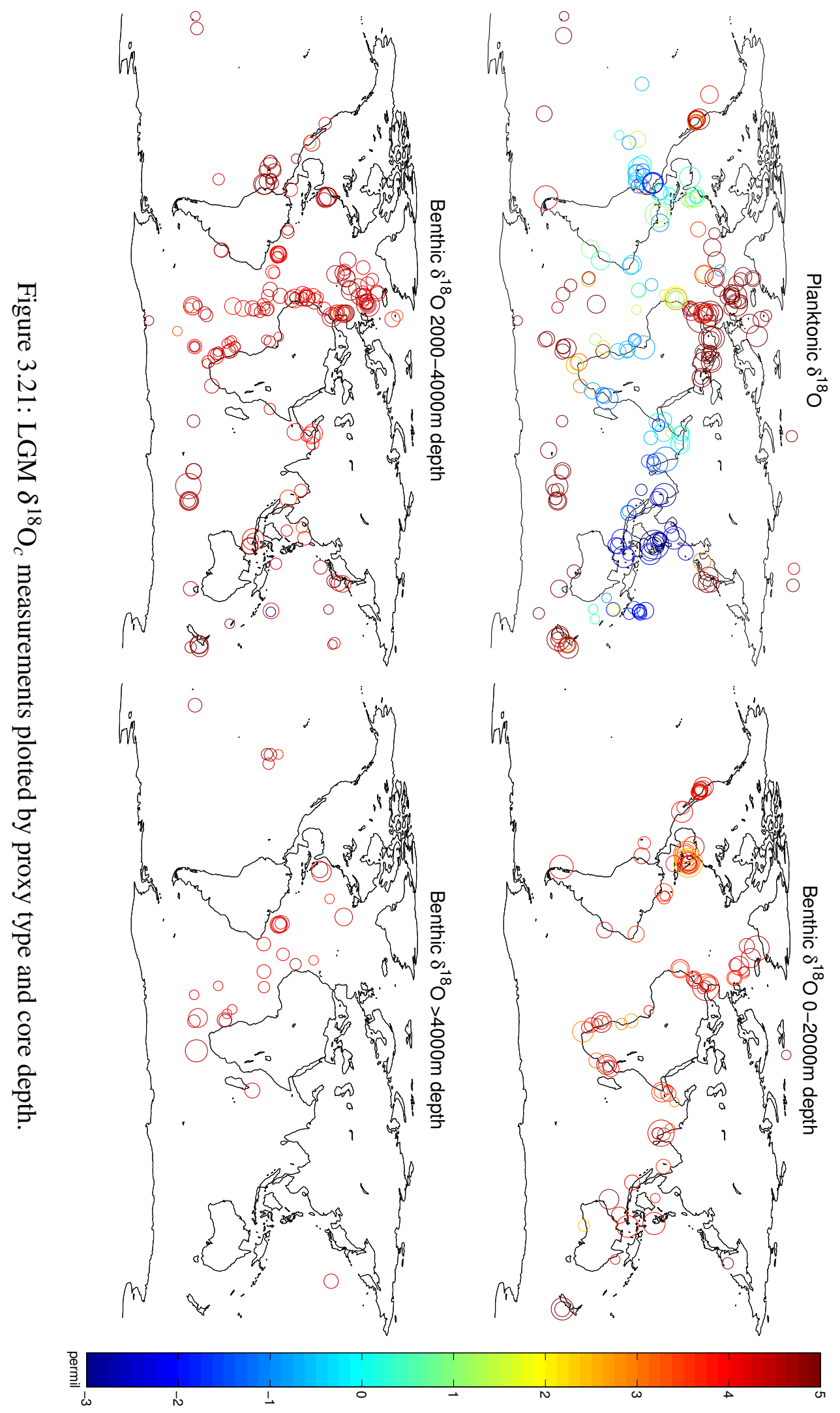



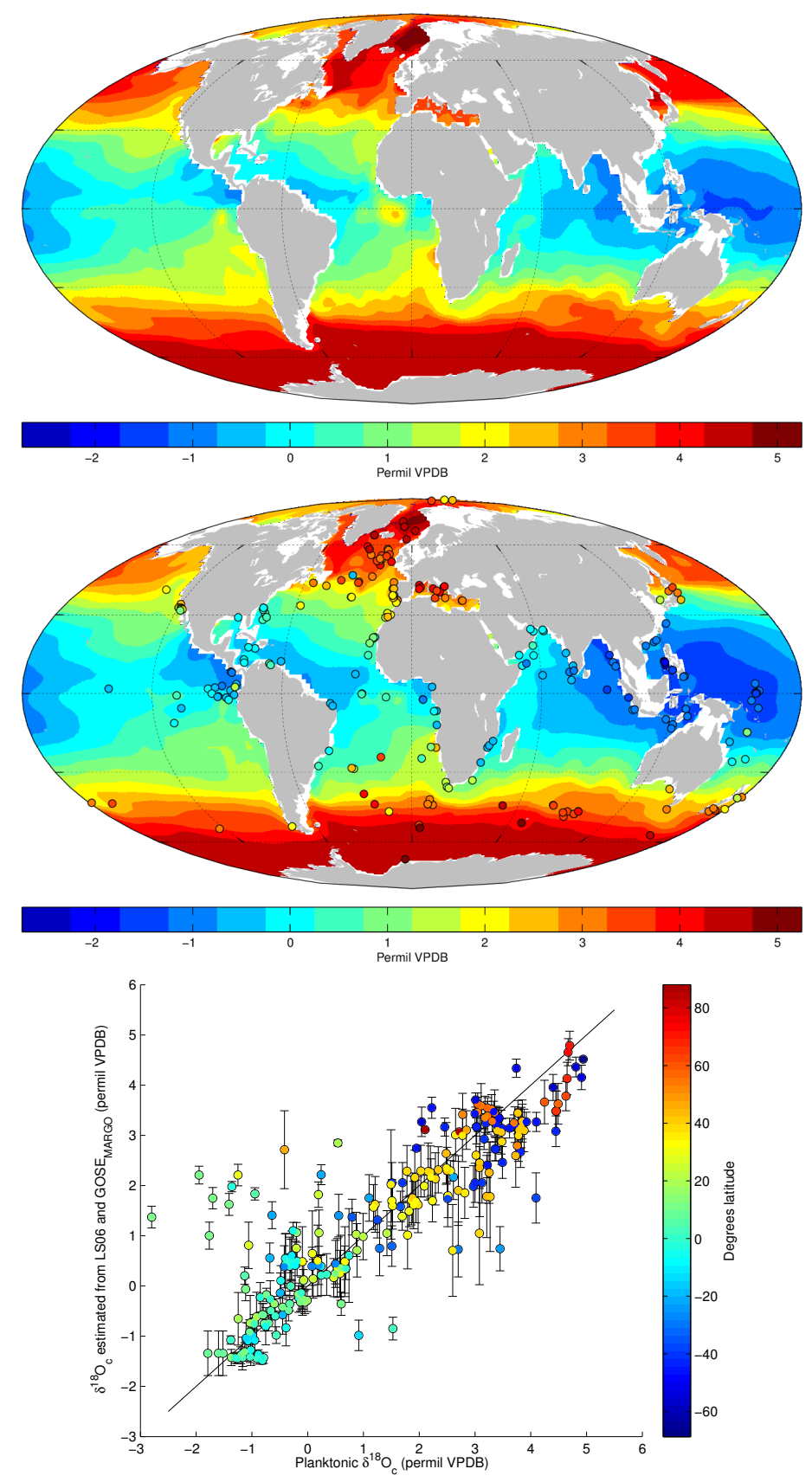

Figure 3.22: Top: Glacial $\delta^{18} \mathrm{O}_{c}$ ML boundary conditions estimated using the coldest monthly average temperatures from $\overline{\mathbf{x}}_{M A R G O}$ (see text). Middle and bottom: Comparison of annual average $\delta^{18} \mathrm{O}_{c}$ derived from $\overline{\mathbf{x}}_{M A R G O}$ with a database of glacial planktonic $\delta^{18} \mathrm{O}_{c}$ measurements. The black line in the bottom panel indicates perfect model-data agreement. Error bars show the range of $\delta^{18} \mathrm{O}_{c}$ arising from seasonal variations as summarized by standard deviations of monthly climatologies at data locations. 


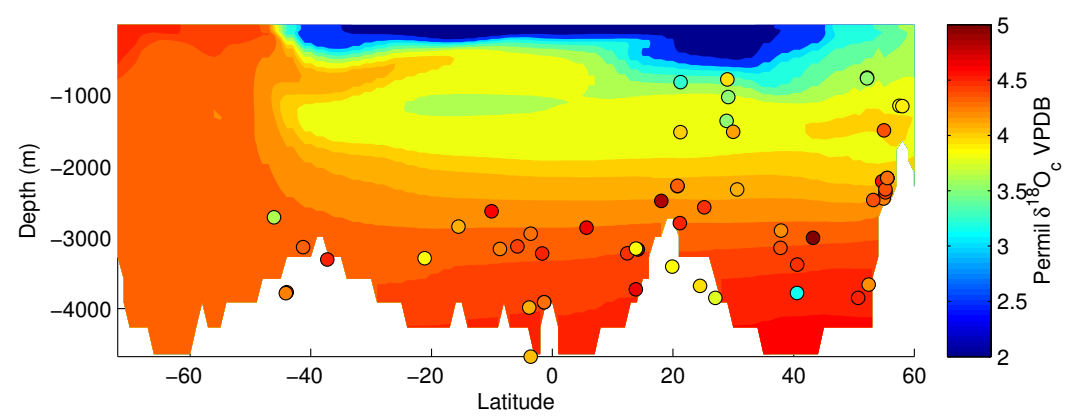

Figure 3.23: Model $\delta^{18} \mathrm{O}_{c}$ averaged zonally over $20^{\circ} \mathrm{W}$ to $10^{\circ} \mathrm{W}$ (a region coincident with many benthic observations, see Figure 3.21) with glacial benthic values overlaid.

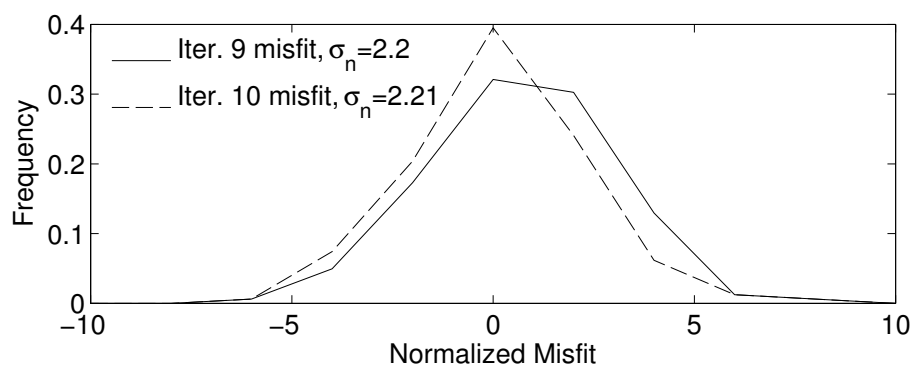

Figure 3.24: Histogram of normalized misfits between the global distribution of benthic $\delta^{18} \mathrm{O}_{c}$ data (Figure 3.21) and the extrapolated equilibrium $\delta^{18} \mathrm{O}_{c}$ simulated in $\overline{\mathbf{x}}_{\text {MARGO }}$ under surface boundary conditions shown in Figure 3.22. Misfits are normalized by a uniform uncertainty of 0.2\% following Marchal and Curry [2008] and Amrhein et al. [2015]. 
solution will depend on the choice of "first guess" (iteration zero) controls. The assumption of a seasonally steady state biases the ways that the model can fit the data, and longer integration times could yield a qualitatively different solution. While work is underway to permit explicit statements of adjoint solution uncertainty [Kalmikov and Heimbach, 2014], here we are unable to make quantitative statements about the solution likelihood. The ad hoc uncertainty quantification procedure of comparing iterations 9 and 10 is instructive but limited, and it seems likely that subsequent iterations or carefully chosen perturbations of the controls could give rise to other qualitatively different solutions with acceptable misfits.

Despite the uncertainties, some simulated features appear to be robust. The ocean surface is inferred to be broadly colder, driven by changes in air temperature and radiation. The barotropic circulation is stronger, consistent with a windier LGM. Both perennial and seasonal sea ice extent is larger, and the Arctic is filled with sea ice year round. Mixed layer depths differ from the modern, with possible implications for abyssal circulation.

These deviations paint a picture of a substantial departure from the modern climate, broadly in line with evidence from various ocean, cryosphere, and terrestrial proxies not explicitly considered in the state estimate. Nevertheless, the MARGO data do not appear to demand that the ocean's role in the climate system departed in some major way from the modern. The simulated LGM ocean still plays a role in poleward heat transport both through abyssal transports and by the northward extensions of western boundary currents. The structure of gyre circulation, while stronger, is largely the same as today.

Several choices were made that differentiate procedures used here from those of DW14 and Dail [2012b], and their influences are evident in the solution. Dail [2012b] described large drifts in 80-year simulations that are not evident in the 100-year simulations described. This is likely because this author began with a first guess model state that was constrained to reproduce observations of the modern ocean over a finite period but that drifted when it was run forward over a longer period. Here we begin with a model that was spun up for 
4,900 years, resulting in much smaller drift. DW14 had a "patchier" solution, with changes to NSSTs most pronounced in regions of high data density; $\overline{\mathbf{x}}_{M A R G O}$ is less patchy because we begin with initial guess forcings from CCSM4, which already have much of the cooling that is necessary to fit the MARGO data imposed over large spatial scales determined by the coupled atmospheric model. A consequence of this choice is that many features of $\overline{\mathbf{x}}_{M A R G O}$ are determined by the CCSM4 forcings, which likely have their own sets of biases. This holds true especially in the deep ocean, which cannot be adjusted much over the 100 year integration period except through changes in initial temperature and salinity. Finally, DW14 used individual proxy observations rather than the MARGO gridded product in the cost function; artifacts in the solution due to the coarseness of the $5^{\circ} \times 5^{\circ}$ grid boxes argue for using the individual proxy measurements in future work, despite the attendant challenges with data uncertainties, or averaging over similar grid boxes in the model when computing the cost function.

The apparent inability of $\delta^{18} \mathrm{O}_{c}$ to constrain abyssal mass and heat transports echoes results of LeGrand and Wunsch [1995] and Huybers et al. [2007], who determined that in order to constrain flow rates from tracer distributions, a "clock" - some measure of the time dependence of the observed tracer field - is necessary. In $\overline{\mathbf{x}}_{M A R G O}$, a clock is present via the primitive equations of the MITgcm, but tracers with additional information about flow speeds or residence times (such as radiocarbon) could prove useful in determining transport rates. A hypothetical tracer experiment in Chapter 4 shows that misfits to abyssal passive tracers are sensitive to flow rates in some parts of the ocean; the limited power of $\delta^{18} \mathrm{O}_{c}$ diagnosed here may chiefly be a result of the noisiness and inconsistency of the data set. If so, it would suggest that those data are better suited to applications where the data are averaged over a large region, thereby mitigating the effects of uncorrelated observational errors.

The presence of a shallower upper AMOC cell, which is reconstructed in both iterations 9 and 10, is in contrast with all of the PMIP3 reconstructions except CCSM4 [Muglia 
and Schmittner, 2015], atmospheric forcings from which were used as the first guess in the state estimate. Running a second state estimate with an alternative first guess (from another PMIP3 model) would be instructive to test the range of abyssal circulations that agree with the MARGO data. Comparison of abyssal stratification to Southern Ocean sea ice extents will permit evaluation of the hypothesis [Ferrari et al., 2014] that sea ice driven buoyancy fluxes play a role in determining abyssal circulation geometry. Several lines of evidence, including studies investigating a closed Bering Strait [Hu et al., 2010] and investigating the role of tidal mixing [Schmittner et al., 2015], as well as the simple argument that larger equator-to-pole atmospheric temperature gradients at the LGM should give rise to windier conditions, argue for a stronger abyssal circulation.

The list of available diagnostics and quantities of interest in an OGCM run is very long, and I have had to be selective in determining what to discuss here. For instance, initial analyses suggest that the global mean stratification was larger, due mostly in changes in salinity. These changes are mostly due to the spin-up under CCSM4 conditions; nevertheless, it is noteworthy that the MARGO data are consistent with a solution that has substantially different stratification from the modern. Southern Ocean sea ice extent is also greater than in the modern, with commensurate effects on mixed layer depths in that region.

Finally, it is worth speculating on the extent to which inferred atmospheric control changes might represent physical atmospheric processes at the LGM, and to what extent such changes are resolvable by the MARGO data. To reiterate, spatiotemporal patterns are not constrained by atmospheric dynamics, and instead reflect data availability and dynamical processes in the upper ocean (though the latter is expected to have a strong effect on the atmosphere). "Patchy" inferred changes in air temperature and radiation are artifacts of the tendency of least-squares solutions that penalize solution norms (as is done here through $J_{c t r l}$ ) to have nonzero solution features only where necessary to fit observations; nevertheless, those changes have the same signs within large regions, consistent with length scales of large-scale 
atmospheric circulation change. Caution is urged in attempting to physically rationalize any inferred control fields too specifically, as different choices of atmospheric controls can have very similar effects on the ocean state, and the partitioning of variance between those fields is in part a function of the prior uncertainty. For instance, similarities between inferred air temperature and surface downwelling radiation suggest changes in those variables are effectively interchangeable and reflects the inability of the data to differentiate between different sources of ocean heating. Overall it seems safe to characterize inferred changes as perturbations to the first guess that are not obviously unphysical, with the caveat that a different set of atmospheric forcings may fit the data equally well and yield a qualitatively different ocean state.

\subsection{Conclusions and future work}

Obtaining a picture of the ocean circulation at the LGM that is consistent with available observations and knowledge of ocean physics is important to understand both the range of climate variability - what might a colder climate look like? - and as the initial conditions for studying the subsequent deglacial transient, when rapid changes in paleoclimate proxy records have been attributed to changes in ocean circulation and chemistry. The least-squares fit of a global dataset of near sea surface observations to the MITgcm is a step towards this goal. In order to fit the data, the ocean state simulated by the MITgcm shows modest deviations from the modern, consistent with a colder climate but inconsistent with hypothesized reorganizations of ocean pathways or dramatic changes in circulation rates.

Priorities for future work include incorporating additional observations, particularly from tracer observations that may be able to constrain the abyssal circulation. Good candidates for additional data include compilations of radiocarbon [Zhao et al., in prep] and stable carbon isotopes; while these tracers are nonconservative and thus more complicated to simulate than 
oxygen isotopes, they benefit from having larger observed gradients in the abyss and from having "clocks" by virtue of their nonconservation. Speculations in this chapter about which regimes (time scales, depths, latitudes) can be constrained by the MARGO network can be tested by an analysis of the adjoint sensitivities to model-data misfits. Ultimately, the goal is to constrain the MITgcm using all possible observations from the LGM data and pose features of the state estimate as hypotheses that can be tested by additional observations. 


\section{Chapter 4}

\section{Ocean state estimation from passive}

\section{tracer observations}

\subsection{Introduction}

This chapter describes and applies techniques for constraining ocean state estimates using passive tracer observations. By contrast with Chapter 2 , the focus here is on estimating seasonally steady circulation, and the model and inverse procedure used are more complex (the method of Lagrange multipliers in the MITgcm, also used in Chapter 3). A practical obstacle to using passive tracer observations is that ocean equilibration times can be very long (up to thousands of years in some regions; Fig. 4.1p, which can make state estimation using the ECCO framework prohibitively computationally expensive. Less complex inverse models can be used used to find steady states at modest computational expense (e.g., LeGrand and Wunsch [1995], Gebbie and Huybers [2006], Gebbie [2014], Marchal and Curry [2008]), but lack seasonal cycles and the more complete representation of ocean dynamics present in OGCMs.

The ability to fit the MITgcm to passive tracer observations using the method of La- 


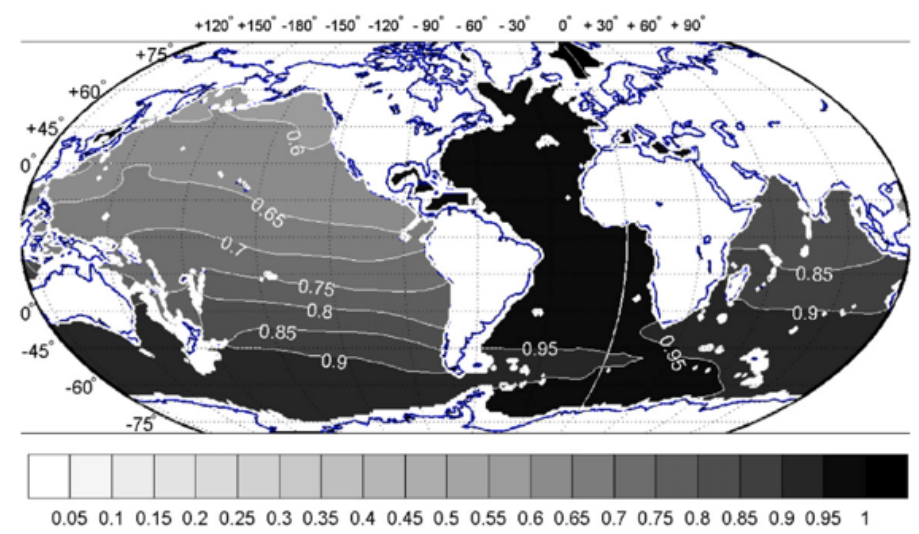

Figure 4.1: Concentration at $1975 \mathrm{~m}$ after 1900 years from a globally uniform surface dye distribution advected in an offline transport simulation derived from ECCO-GODAE; figure from Wunsch and Heimbach [2008]. The system is far from equilibrium (where the concentration everywhere is 1) in large parts of the Indian and Pacific Oceans.

grange multipliers is developed and demonstrated in idealized sensitivity experiments evaluating what model changes cause model Antarctic Bottom Water to shoal. The analyses rely on two technical contributions outlined in this chapter: 1) a generalization of the Bryan [1984] asynchronous time stepping scheme that further accelerates tracer equilibration (Section 4.3.2), and 2) an extrapolation procedure that usefully predicts tracer equilibrium values in a numerical model long before equilibrium is actually reached (Section 4.3.3).

\subsection{Background}

\subsubsection{Inferences from tracers at the LGM}

Current perspectives on the LGM abyssal ocean are shaped largely by proxy observations of passive ocean tracers. For instance, a leading interpretation of data from Atlantic suggests that during the LGM, abyssal waters formed in the North Atlantic (an analog of modern North Atlantic Deep Water (NADW)) shoaled and that deep water from the Southern Ocean filled more of the deep ocean [e.g., Curry et al., 1988, Duplessy et al., 1988, Marchitto et al., 2002, Curry and Oppo, 2005, Lynch-Stieglitz et al., 2007]. Support for these conclusions 
is rooted in semi-conservative proxies for nutrient concentration, such as $\delta^{13} \mathrm{C}$, although some studies have argued [e.g., LeGrand and Wunsch, 1995] that different nutrient concentrations (for instance, from changing biological productivity or remineralization) might produce a similar tracer distribution given a modern-like mass circulation. Exactly what physical changes could cause the different observed LGM tracer distributions are not clear [Huybers et al., 2007].

More generally, while numerous geochemical tools have been developed to constrain abyssal LGM circulation, significant uncertainties remain, and our understanding of LGM circulation is far from complete. A body of evidence suggests that abyssal circulation rates were reduced and deep ocean residence times were increased at the LGM, at odds with the notion of a more vigorous circulation driven by larger wind stress and tidal dissipation [Wunsch, 2003] and the results of Chapter 3 . Keigwin and Schlegel] [2002] argued that ventilation ages (the time since a parcel was exposed to the atmosphere, measured in that study by ${ }^{14} \mathrm{C}$ ages) in the western North Atlantic during several time slices within the LGM (defined as 21-18 kya) were roughly five times as great as those found in modern NADW, suggesting a longer parcel residence time. McManus et al. [2004] measured ratios of ${ }^{213} \mathrm{~Pa} /$ ${ }^{230} \mathrm{Th}$ excess (which includes a time component from the respective scavenging rates of the two isotopes by particles in the ocean) at Bermuda Rise and postulated a slightly reduced AMOC upper strength during the LGM, though subsequent work has revealed problems with the interpretation of ${ }^{213} \mathrm{~Pa} /{ }^{230} \mathrm{Th}$ [e.g., Burke et al., 2011, Hayes et al., 2015]. LynchStieglitz et al. [1999] assumed a relationship between density and the oxygen isotope ratio $\delta^{18} \mathrm{O}_{c}$ measured in benthic foraminifera and used the thermal wind relationship to infer a reduced LGM circulation. However, their assumption of a level of no motion may have been flawed [Wunsch, 2003] and variations in the relationship between $\delta^{18} \mathrm{O}_{c}$ and density [e.g. Gebbie and Huybers, 2006] complicate the picture further.

Reconciling these observations with one another and with the relevant dynamics would 
be a major step in understanding LGM abyssal circulation. Several studies have combined paleoceanographic measurements and physical models to impose explicit physical constraints on inferences of the ocean state during the LGM [LeGrand and Wunsch, 1995. Winguth et al., 2000, Rutberg and Peacock, 2006, Huybers et al., 2007, Gebbie and Huybers, 2006, Marchal and Curry, 2008, Burke et al., 2011, Lund et al., 2011, Dail, 2012a, Gebbie, 2014]. A brief summary of these studies follows. In all cases, the model physics is steady and consists of some combination of mass and tracer conservation, planetary vorticity conservation, and thermal wind relationships. Many of the studies seek to disprove the null hypothesis $\left(\mathrm{H}^{0}{ }_{\text {mod }}\right.$, first introduced in LeGrand and Wunsch [1995] and a focus of Chapter 2) that the paleo observations are consistent with the modern ocean circulation.

- LeGrand and Wunsch [1995] used a $10 \times 10$ degree model of the deep North Atlantic with $1 \mathrm{~km}$ vertical resolution, thermal wind balance, and conservation of mass, tracers, and vorticity. They concluded that LGM estimates of oxygen and carbon isotopes could not constrain transport rates in the model.

- Winguth et al. [2000] used the method of Lagrange multipliers with a $3.5 \times 3.5$ degree global geostrophic model that had simple sea ice and biological pump processes. They found that LGM benthic $\delta^{13} \mathrm{C}$ and $\mathrm{Cd} / \mathrm{Ca}$ data are consistent with a model ocean state having transport in an NADW analog that is half as strong as estimated in the modern ocean.

- Gebbie and Huybers [2006] used $\delta^{18} \mathrm{O}_{c}$ as a proxy for density and inferred LGM meridional transports along a zonal section in the South Atlantic using a two-dimensional 1 degree geostrophic model with $120 \mathrm{~m}$ vertical resolution. They found that LGM observations could reject $\mathrm{H}^{0}{ }_{\text {mod }}$ only if assumptions were made about LGM temperature and salinity distributions and if one assumed a spatially stationary relationship between 
salinity and the $\delta^{18} \mathrm{O}$ of seawater.

- Marchal and Curry [2008] used a $2 \times 2$ degree geostrophic model of the abyssal Atlantic Ocean with vertical resolution varying between $1000 \mathrm{~m}$ and $1500 \mathrm{~m}$. They compiled a set of benthic $\delta^{18} \mathrm{O}_{c}$ and $\delta^{13} \mathrm{C}$ observations and demonstrated that about $20 \%$ of LGM $\delta^{13} \mathrm{C}$ data in the Atlantic are inconsistent with modern estimates, but only if sediment core measurement error is less than $0.1 \%$ and mixing processes have a negligible influence on the transport of $\delta^{13} \mathrm{C}$.

- Burke et al. [2011] used a $4 \times 4$ degree model of the Atlantic abyssal ocean with 1000 $\mathrm{m}$ vertical resolution and found that a compilation of ${ }^{213} \mathrm{~Pa} /{ }^{230} \mathrm{Th}$ measurements from the Holocene, LGM, and Heinrich Event 1 did not provide disprove $\mathrm{H}^{0}{ }_{\text {mod }}$ for those intervals.

- Lund et al. [2011] used a box model and LGM measurements of $\delta^{18} \mathrm{O}$ and $\delta^{13} \mathrm{C}$ to constrain ratios of advection and diffusion. They proposed that shoaling of watermass boundaries away from regions of vigorous mixing at the depth of the Mid-Atlantic Ridge could explain observations of increased LGM vertical tracer gradients.

- Gebbie [2014] found a watermass pathways solution consistent with several hundred $\delta^{18} \mathrm{O}, \delta^{13} \mathrm{C}$, and $\mathrm{Cd} / \mathrm{Ca}$ observations at the LGM that indicates a shoaling of the boundary between NADW and AABW. If assumptions are made about the $\delta^{13} \mathrm{C}$ exported from the Southern Ocean, then Gebbie [2014] is able to reject $\mathrm{H}^{0}{ }_{\text {mod }}$.

In contrast to these studies using models with simplified dynamics, Dail [2012b] used a primitive equation model (the MITgcm) and the method of Lagrange multipliers (Section 3.2.3) to fit abyssal observations of $\delta^{18} \mathrm{O}$ and $\delta^{13} \mathrm{C}$ in the Atlantic, but inferences from those data were limited by computational expense. This chapter extends that work by introducing 
numerical tools that accelerate equilibration of tracer distributions in OGCMs so that modeldata misfit for passive tracer observations can be computed more efficiently.

\subsubsection{Challenges of seasonally quasi-steady ocean state estimation us- ing passive tracer observations}

Motivations for using passive tracer observations in the ECCO framework arise because (1) the primitive equations introduce more dynamical information that can make paleoceanographic inverse problems better constrained, and (2) simulating changes in the ocean state that obey those equations may yield new physical insights. Ideally, the procedure would be similar to those used with simpler ocean circulation models, where a steady solution to the governing equations is found that best fits the data in a least-squares sense. Unfortunately, numerical tools to solve directly for seasonally steady solutions to OGCMs are unavailable, and are not likely to be developed for the foreseeable future. Instead, the typical procedure (e.g. Braconnot et al. [2012]) is to integrate an OGCM forward in time for an interval $\tau_{s}$ until the system has nearly equilibrated. In the absence of computational constraints, one could adopt a similar procedure in state estimation, integrating the MITgem forward over a spin-up interval $\tau_{s}$ and computing the model-data misfit $J_{\text {data }}$ over a subsequent interval. Lagrange multipliers computed for this integration would give sensitivities of the misfit at quasi-equilibrium to the climatological controls, and the procedure could be iterated until a cost minimum was achieved.

In practice, integrating the model over a long enough interval $\tau_{s}$ (thousands or tens of thousands of years of years) such that it is nearly equilibrated at every iteration is too computationally onerous, and one is restricted to using shorter spin-up intervals. Two major shortcomings of this procedure are (1) biases in the model-data misfit because of model disequilibrium and (2) exclusion of processes with time scales longer than $\tau_{s}$ to adjust the model 
to the data. Figure (4.3) illustrates how model-data misfit bias is manifest in passive tracers. Problems stemming from excluding long time scale processes are illustrated by Dail [2012b], who constructed a modern ocean state estimate constrained in part by measurements of the carbon isotope ratio of total dissolved inorganic carbon $\left(\delta^{13} \mathrm{C}_{\text {DIC }}\right)$. Figure 4.2 illustrates a solution that fits the $\delta^{13} \mathrm{C}_{\mathrm{DIC}}$ data within their uncertainties but has patchiness in modeled $\delta^{13} \mathrm{C}_{\text {DIC }}$ arising from inferred changes in the tracer initial conditions, which were the only part of the control vector able to reduce model-data misfit on time scales of the model simulation (20 years). These features appear to suggest strong, localized interior sources for this tracer, which are improbable in the deep ocean, where it is nearly conservative [Marchal and Curry, 2008].

To address these challenges, Dail [2012b] proposed using asynchronous time stepping (ATS, discussed further below), and carry-over control, whereby increasingly longer state estimates are performed in sequence. The carry-over control technique was shown to reduce model drift in longer simulations, improving the model's ability to reduce model-data misfit. Despite these advances, a SQSE could not be found that fit the model to the OCCA ocean atlas [Forget, 2010]; in particular, the solution drifted strongly. It was hypothesized that this inability could be due to inadequacies in the model or observations; the necessity for including a broader spectrum of variability beyond the seasonal cycle to include sub- or interannual variability; or the inability of a steady state circulation to reproduce observations of the modern ocean, possibly because of strong disequilibria between the modern ocean and atmosphere. 


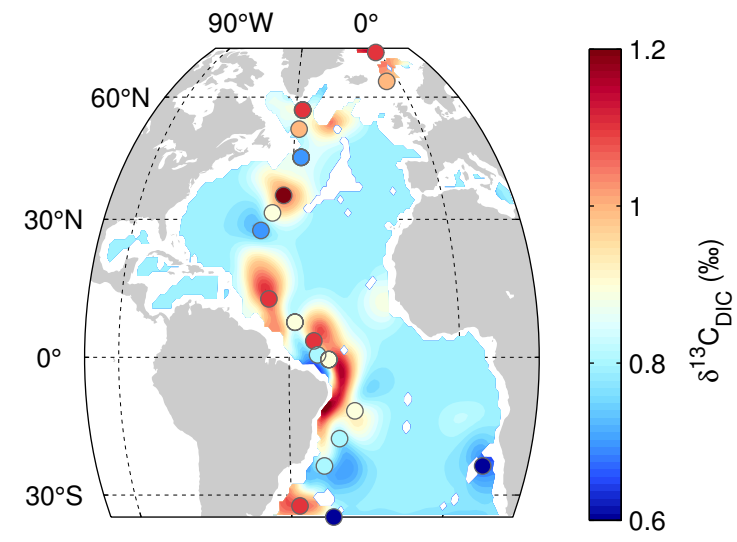

Figure 4.2: Comparison of the carbon isotope ratio of dissolved organic carbon $\delta^{13} \mathrm{C}_{\mathrm{DIC}}$ at model depth $2730 \mathrm{~m}$ (colored contours) in a 10-year state estimate from Dail [2012b] (her Figure 3-17) with modern GEOSECS $\delta^{13} \mathrm{C}_{\text {DIC }}$ observations. The modeled distribution of $\delta^{13} \mathrm{C}_{\text {DIC }}$ is patchy.

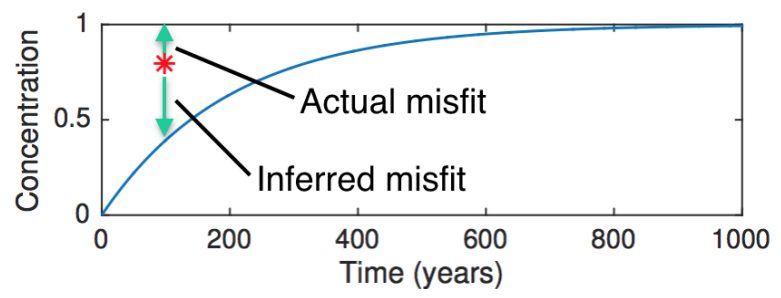

Figure 4.3: Misfits between observations and model simulated tracer concentrations can be biased for disequilibrated model tracer states. Here when a tracer observation (red star) assumed to represent an equilibrated system is compared to a hypothetical grid box tracer concentration trajectory (blue line) after 100 years, the sign of the misfit is incorrect. 


\subsection{Procedures for accelerating passive tracer equilibra- tion for use in state estimation}

This section describes modeling approaches for accelerating and extrapolating tracer equilibration towards the goal of using passive tracers in ocean state estimation. Asynchronous time stepping is used in the momentum and tracer equations, allowing for faster simulations, and vertically asynchronous time steps are used in addition for the passive tracers. A tracer acceleration approach that generalizes the level-by-level approach of Bryan [1984] is derived as a matrix preconditioning operation; this approach yields a scaling for the vertically asynchronous passive tracer time steps. A tracer extrapolation procedure is also developed. The longer simulations afforded by asynchronous time stepping and the ability to penalize deviations from estimated equilibrium tracer values mitigate the shortcomings (discussed in the previous section) of using model integrations that are short relative to modern spin-up times. These procedures are explored in simple models here and tested in a more complex GCM in the following section.

\subsubsection{Asynchronous time stepping}

Bryan [1984] described a numerical scheme for accelerating the convergence of ocean GCMs by introducing depth-dependent scalar factors $\alpha(z)$ and $\gamma(z)$ into ocean momentum and tracer equations,

$$
\begin{aligned}
\alpha(z) \frac{\partial \mathbf{u}}{\partial t} & =F \\
\gamma(z) \frac{\partial(\theta, S, C)}{\partial t} & =G .
\end{aligned}
$$

Here $z$ is depth, $\mathbf{u}$ is horizontal velocity, $t$ is time, $\theta$ is potential temperature, $S$ is salinity, $C$ 
is a passive tracer, and $F$ and $G$ are the remaining (forcing) terms in each equation. Different values for $\alpha(z)$ and $\gamma(z)$ serve to accelerate different parts of the ocean domain, either by stretching the model time step or changing the local heat and salt capacities at a particular depth level. When $\alpha(z)=\gamma(z)=1$, the normal equations using synchronous time-stepping are recovered. When these parameters are less than 1, Equations 4.1 and 4.2 describe a distorted physics that narrows the frequency band of ocean model variability by reducing Rossby and Kelvin wave speeds while shrinking very long time scales of advection and diffusion in the abyss. Because $\alpha(z)$ and $\gamma(z)$ only affect local time derivatives, equilibrium ocean solutions under truly steady forcing (which are obtained by setting $\partial / \partial t$ terms to zero) are analytically identical under distorted physics. Studies employing seasonally steady forcing have also produced satisfactory results, with some caveats (discussed below). This approach has been used in many contexts to speed equilibration in ocean models as well as coupled atmosphere-ocean GCMs. The success of the scheme ultimately relies on the dominance of geostrophic balance in the oceans, whereby the momentum equations adjust rapidly to pressure gradients and vice versa [Hecht, 2006].

Following Dail [2012b] and Bugnion and Hill [2006], I use ATS in the temperature and salinity equations by setting $\alpha<1$. Dail [2012b] showed that use of $\alpha<1$ has a minor effect on the final state estimate. Danabasoglu et al. [1996] and Wang [2001] note that in forward simulations there can be distortion of seasonal cycles resulting from ATS, but that most characteristics of the seasonal cycle can be recovered by integrating over a short period (several decades) using synchronous time steps after an asynchronous equilibration. 


\subsubsection{Jacobi preconditioning to accelerate tracer convergence and pro- vide a scaling for vertically asynchronous time stepping}

This subsection extends the Bryan [1984] approach by considering horizontal variations in time steps (as opposed to strictly depth-dependent procedures described in Bryan [1984]), which increases the rate of convergence whenever fluxes or grid box volumes are nonuniform within depth levels. Work is underway to implement this procedure in the MITgcm, and may prove useful in future work implementing nonconservative tracers (such as radiocarbon) where the extrapolation methods described in Section 4.3 .3 fail. The scaling for $\gamma(z)$ that emerges from this work is used for vertically-varying time steps (the Bryan [1984] scheme) in the MITgem forward and adjoint simulation discussed in Sections 4.4 and 4.5 .

Related approaches taken to improve convergence rates include using implicit time steps [Bardin et al., 2014], so-called matrix methods, which rely on constructing a state transition matrix from a steady flow regime [Khatiwala et al., 2005, Khatiwala, 2007], and NewtonKrylov preconditioning [Li and Primeau, 2008, Khatiwala, 2008]. The method described here accelerates convergence but does not require construction of the state transition matrix or use of an implicit solver, neither of which is feasible in the MITgcm adjoint. Instead, time steps are scaled in model grid boxes so that the Courant number $\mathrm{Cr}$ is 1 everywhere; this scaling can be interpreted as applying a Jacobi preconditioner.

\subsubsection{Development}

Define the system state vector $\mathbf{c}(t)$ to be the list of tracer concentrations in every model grid box of an OGCM. The state evolution under concentration boundary conditions $\mathbf{q}(t)$ is a solution to linear, constant-coefficient, inhomogeneous system of first-order differential 
equations that can be written

$$
\frac{d}{d t} \mathbf{c}(t)=\mathbf{A c}(t)+\mathbf{F q}(t)
$$

The matrices $\mathbf{A}$ and $\mathbf{F}$ are determined by the flow and model geometry and can be written in terms of grid box volumes, $V_{i}$, and fluxes, $\mathbf{J}_{i j}$, representing the positive volume transport from the $i^{t h}$ to the $j^{t h}$ grid box due to both advective and diffusive processes [Wunsch, 2003],

$$
\begin{aligned}
\mathbf{A}_{i i} & =-\frac{\sum_{j \in N_{i}} \mathbf{J}_{i j}}{V_{i}} \\
\mathbf{A}_{i j} & =\frac{\mathbf{J}_{j i}}{V_{i}}, i \neq j \\
\mathbf{F}_{k i} & =\frac{\mathbf{J}_{k i}}{V_{i}}
\end{aligned}
$$

The set $N_{i}$ is the neighborhood of points adjacent to the $i^{\text {th }}$ grid box. Equation 4.3) can be discretized in time by finite differences as

$$
\begin{aligned}
\frac{\mathbf{c}(t+\Delta t)-\mathbf{c}(t)}{\Delta t} & =\mathbf{A c}(t)+\mathbf{F} \mathbf{q}_{0} \\
\mathbf{c}(t+\Delta t) & =(\mathbf{I}+\mathbf{A} \Delta t) \mathbf{c}(t)+\mathbf{F} \Delta t \mathbf{q}_{0}
\end{aligned}
$$

The system equilibrium state, $\mathbf{c}_{0}$, can be found by computing the inverse of $\mathbf{A}$

$$
\begin{aligned}
\mathbf{c}_{0} & =(\mathbf{I}+\mathbf{A} \Delta t) \mathbf{c}_{0}+\mathbf{F} \Delta t \mathbf{q}_{0} \\
-\mathbf{A} \Delta t \mathbf{c}_{0} & =\mathbf{F} \Delta t \mathbf{q}_{0} \\
\mathbf{c}_{0} & =(-\mathbf{A})^{-1} \mathbf{F} \mathbf{q}_{0} .
\end{aligned}
$$

Existence of $\mathbf{c}_{0}$ depends on the invertibility of $\mathbf{A}$. Construction of $\mathbf{A}$ and computation of $(-\mathbf{A})^{-1}$ explicitly is often technically and computationally challenging and is not usable in 
the MITgcm adjoint.

Approximations to $\mathbf{c}_{0}$ can be found by truncating the geometric series expansion for $-\mathbf{A}^{-1}$ which is equivalent to running a tracer transport model for a finite number of time steps. Iterating Equation (4.8) gives the system state after $k$ time steps,

$$
\mathbf{c}(k \Delta t)=\left[\mathbf{I}+(\mathbf{I}+\mathbf{A})+(\mathbf{I}+\mathbf{A})^{2}+\cdots+(\mathbf{I}+\mathbf{A})^{k-1}\right] \mathbf{F} \mathbf{q}_{0} .
$$

By the geometric series expansion for $-\mathbf{A}^{-1}$,

$$
(-\mathbf{A})^{-1}=\sum_{n=0}^{\infty}(\mathbf{I}+\mathbf{A})^{n}
$$

the iterations will eventually converge to $\mathbf{c}_{0}$,

$$
\lim _{k \rightarrow \infty} \mathbf{c}(k \Delta t)=\sum_{n=0}^{\infty}(\mathbf{I}+\mathbf{A})^{n} \mathbf{F} \mathbf{q}_{0}=\mathbf{c}_{0} .
$$

A general solution for Equation 4.3 can also be written in terms of the eigenvectors and eigenvalues of $\mathbf{A}$,

$$
\mathbf{c}(t)=\sum_{m=1}^{M} \kappa_{m} e^{\lambda_{m} t} \mathbf{v}_{m}+\mathbf{c}_{0} .
$$

where $\lambda_{m}$ and $\mathbf{v}_{m}$ are the eigenvalues and eigenvectors, respectively, and the $\kappa_{m}$ are arbitrary constants. For a stable system, the transient contributions must decay exponentially in time $\left(\lambda_{m} \leq 0\right.$ for all $\left.m\right)$. Convergence to $\mathbf{c}_{0}$ within a $5 \%$ tolerance in large advective-diffusive systems such as ocean GCMs can take hundreds or thousands of years model, depending on the geographical region of interest (Wunsch and Heimbach [2008]), implying some $\left|\lambda_{m}\right| \ll 1$.

Preconditioning scales the matrices $\mathbf{A}$ and $\mathbf{F}$ so that the equilibrium solution is the same but convergence to equilibrium through the time-stepping scheme (whether by computation of matrix powers (Equation (4.8) or by a GCM) is faster. The goal is to make the $\lambda_{m}$ as neg- 
ative as possible. Left multiplying Equation 4.10 by an invertible matrix $\overline{\mathbf{P}}$ and rearranging gives

$$
\overline{\mathbf{P}} \mathbf{A} \Delta t \mathbf{c}_{0}+\overline{\mathbf{P}} \mathbf{F} \Delta t \mathbf{q}_{0}=\mathbf{0}
$$

This left matrix multiplication changes the appearance of the problem, but it does not change the equilibrium distribution, $\mathbf{c}_{0}$. To demonstrate this, define $\mathbf{A}^{\prime}=\overline{\mathbf{P}} \mathbf{A}$ and $\mathbf{F}^{\prime}=\overline{\mathbf{P}} \mathbf{F}$ and let $\mathbf{c}_{0}^{\prime}$ be the equilibrium solution in the preconditioned system. Then Equation (4.16) can be written $-\mathbf{A}^{\prime} \Delta t \mathbf{c}_{0}^{\prime}=\mathbf{F}^{\prime} \Delta t \mathbf{q}_{0}$, the same form as Equation (4.10) and having solution $\mathbf{c}_{0}^{\prime}=$ $\left(-\mathbf{A}^{\prime}\right)^{-1} \mathbf{F}^{\prime} \mathbf{q}_{0}$. Substituting primed variables,

$$
\begin{aligned}
\mathbf{c}_{0}^{\prime} & =\left(-\mathbf{A}^{\prime}\right)^{-1} \mathbf{F}^{\prime} \mathbf{q}_{0} \\
& =(-\overline{\mathbf{P}} \mathbf{A})^{-1} \overline{\mathbf{P}} \mathbf{F} \mathbf{q}_{0} \\
& =(-\mathbf{A})^{-1} \overline{\mathbf{P}}^{-1} \overline{\mathbf{P}} \mathbf{F} \mathbf{q}_{0} \\
& =(-\mathbf{A})^{-1} \mathbf{F} \mathbf{q}_{0} \\
& =\mathbf{c}_{0}
\end{aligned}
$$

Numerous choices for $\overline{\mathbf{P}}$ have been studied to improve convergence of iterative solutions to problems of the form $\mathbf{A x}=\mathbf{b}$ [Strang and Aarikka, 1986]; here we select the Jacobi preconditioner, written as $\mathbf{P}$, which has a simple physical interpretation. (For a broad introduction to applications of preconditioning in fluid dynamics, see Turkel[[1993]). The Jacobi preconditioner is the diagonal matrix whose diagonal elements are the inverse of the diagonal elements of $-\mathbf{A} \Delta t$,

$$
\mathbf{P}^{-1}=\operatorname{diag}(-\mathbf{A} \Delta t)
$$

so that

$$
\mathbf{P}_{i i}=\frac{V_{i}}{\sum_{j \in N_{i}} \mathbf{J}_{i j} \Delta t}=\frac{1}{C r_{i}}
$$


where $C r_{i}$ is the Courant number in each model grid box. If any diagonal elements of $\mathbf{A} \Delta t$ are zero, the preconditioner is undefined.

Left preconditioning has the effect of row-wise multiplying $\mathbf{A} \Delta t$ and $\mathbf{F} \Delta t$ by the diagonal elements $\mathbf{P}_{i i}=1 / C r_{i}$ of the preconditioning matrix,

$$
\begin{aligned}
& {[\mathbf{P A} \Delta t]_{i i}=-\frac{1}{C r_{i}}\left(\frac{\sum_{j} \mathbf{J}_{i j}}{V_{i}} \Delta t\right)} \\
& {[\mathbf{P A} \Delta t]_{i j}=\frac{1}{C r_{i}}\left(\frac{\mathbf{J}_{j i}}{V_{i}} \Delta t\right), i \neq j} \\
& {[\mathbf{P F} \Delta t]_{k i}=\frac{1}{C r_{i}}\left(\frac{\mathbf{J}_{k i}}{V_{i}} \Delta t\right) .}
\end{aligned}
$$

Per Equations (4.24)-(4.26), Jacobi preconditioning is equivalent to scaling the volumes of every grid box, or the fluxes out of every grid box, or the time step in every grid box. We adopt the latter way of thinking because it is easiest to implement in an ocean GCM, and define

$$
\mathbf{P}_{i i}=\frac{\Delta t_{i}^{\prime}}{\Delta t} .
$$

where $\Delta t_{i}^{\prime}$ are the new grid box-specific time steps. Combining with Equation (4.23),

$$
\begin{aligned}
\frac{\Delta t_{i}^{\prime}}{\Delta t} & =\frac{1}{C r_{i}} \\
\Delta t_{i}^{\prime} & =\frac{\Delta t}{C r_{i}}=\frac{V_{i}}{\sum_{j \in N_{i}} \mathbf{J}_{i j}}
\end{aligned}
$$

Jacobi preconditioning scales time steps in every grid box by $1 / C r_{i}$ so that the new time steps $\Delta t_{i}^{\prime}$ are equal to the grid box flushing times $V_{i} / \sum_{j} \mathbf{J}_{i j}$. The new Courant numbers,

$$
C r_{i}^{\prime}=\frac{\sum_{j \in N_{i}} \mathbf{J}_{i j}}{V_{i}} \Delta t^{\prime},
$$


obey $C r_{i}^{\prime}=1$ for all $i$. In an ocean GCM, rapid convergence can be achieved by choosing time steps satisfying Equation (4.29), and $\mathbf{A}$ and $\mathbf{F}$ need not be computed or stored.

Combining Equations (4.24) and (4.28), the diagonal elements $[\mathbf{P A}]_{i i}$ obey

$$
\begin{aligned}
{[\mathbf{P A}]_{i i} } & =-\frac{\sum_{j} \mathbf{J}_{i j}}{V_{i}} \frac{V_{i}}{\sum_{j} \mathbf{J}_{i j}} \\
& =-1 .
\end{aligned}
$$

As any diagonal values less than -1 would violate the CFL condition, the choice of time scaling in Equation (4.29) makes the trace of PA as negative as possible. This is relevant because, defining $\lambda_{m}^{\prime}$ to be the eigenvalues of $\mathbf{P A}$,

$$
\operatorname{tr}(\mathbf{P A})=\sum_{m} \lambda_{m}^{\prime} .
$$

Because $\lambda_{m}^{\prime}<0$ for all $m$, Equation 4.29 is also the choice of time step change that maximizes $\sum_{m}\left|\lambda_{m}^{\prime}\right|$, which is one metric for the convergence of the matrix power series. However, small values of $\left|\lambda_{m}^{\prime}\right|$ may still remain, and rapid convergence to equilibrium is not guaranteed.

\subsubsection{Demonstration in a four-box model}

Here we illustrate the benefits of preconditioning on the four box model used in Chapter 2. Model volumes and fluxes (Figure (4.4)) correspond in an order-of-magnitude sense to the geometry and vertical transports of the modern North Atlantic [Ganachaud and Wunsch. [2000]. Fluid with steady tracer values equal to $q_{M}=1$ and $q_{H}=0$ is transported at constant rates $F_{M}=1 \mathrm{~Sv}$ and $F_{H}=9 \mathrm{~Sv}$ from middle- and high-latitude ML boxes, respectively, to an intermediate box with volume $V_{1}=10^{8} \mathrm{~km}^{3}$ and tracer concentration equal to $c_{1}(t)$. The intermediate box in turn exchanges fluid at constant rate $F_{12}=10 \mathrm{~Sv}$ with a deep box with 
volume $V_{2}=10^{8} \mathrm{~km}^{3}$ and tracer concentration $c_{2}(t)$. A compensating flux, $F_{2 \infty}=10 \mathrm{~Sv}$, evacuates fluid from the deep box out of the system.

The state vector is $\mathbf{c}(t)=\left[c_{1}(t), c_{2}(t)\right]^{\top}$ and the ML vector is $\mathbf{q}(t)=\left[q_{M}(t), q_{H}(t)\right]^{\top}$. Combining the equations for conservation of volume and tracer describes the evolution in each box as a vector equation in the form of Eq. 4.8,

$$
\mathbf{c}(t+\Delta t)=\left[\begin{array}{cc}
1-\frac{\Delta t}{\tau_{1}} & 0 \\
\frac{F_{12} \Delta t}{V_{2}} & 1-\frac{\Delta t}{\tau_{2}}
\end{array}\right] \mathbf{c}(t)+\left[\begin{array}{cc}
\frac{F_{H} \Delta t}{V_{1}} & \frac{F_{M} \Delta t}{V_{1}} \\
0 & 0
\end{array}\right] \mathbf{q}(t)
$$

where the flushing times, $\tau_{1}$ and $\tau_{2}$, are defined as $\tau_{1}=V_{1} /\left(F_{M}+F_{H}\right)$ and $\tau_{2}=V_{2} /\left(F_{M}+F_{H}\right)$; here $\tau_{1}=\tau_{2}=317$ years.

Figure (4.4) shows equilibration in the intermediate and deep boxes (in both cases, the equilibrium values are 0.1 ) from a zero initial state in the preconditioned (scaled time steps) and non-preconditioned cases. In non-preconditioned cases, time steps of 20 years are used, and there is an exponential approach to equilibrium, with $90 \%$ equilibration at 37 time steps in the intermediate box and 61 time steps in the deep box. In the preconditioned cases, the effective time steps are 317 years (the flushing times in each box). The intermediate box is equilibrated immediately (after the first time step), while the deep box is equilibrated after two time steps. An additional time step is required for the deep box because at the model initial time the intermediate box, which lies "upstream," has not yet equilibrated. More generally, the time to equilibrium under this choice of preconditioning is limited by the connectivity of grid boxes rather than their flushing times. 

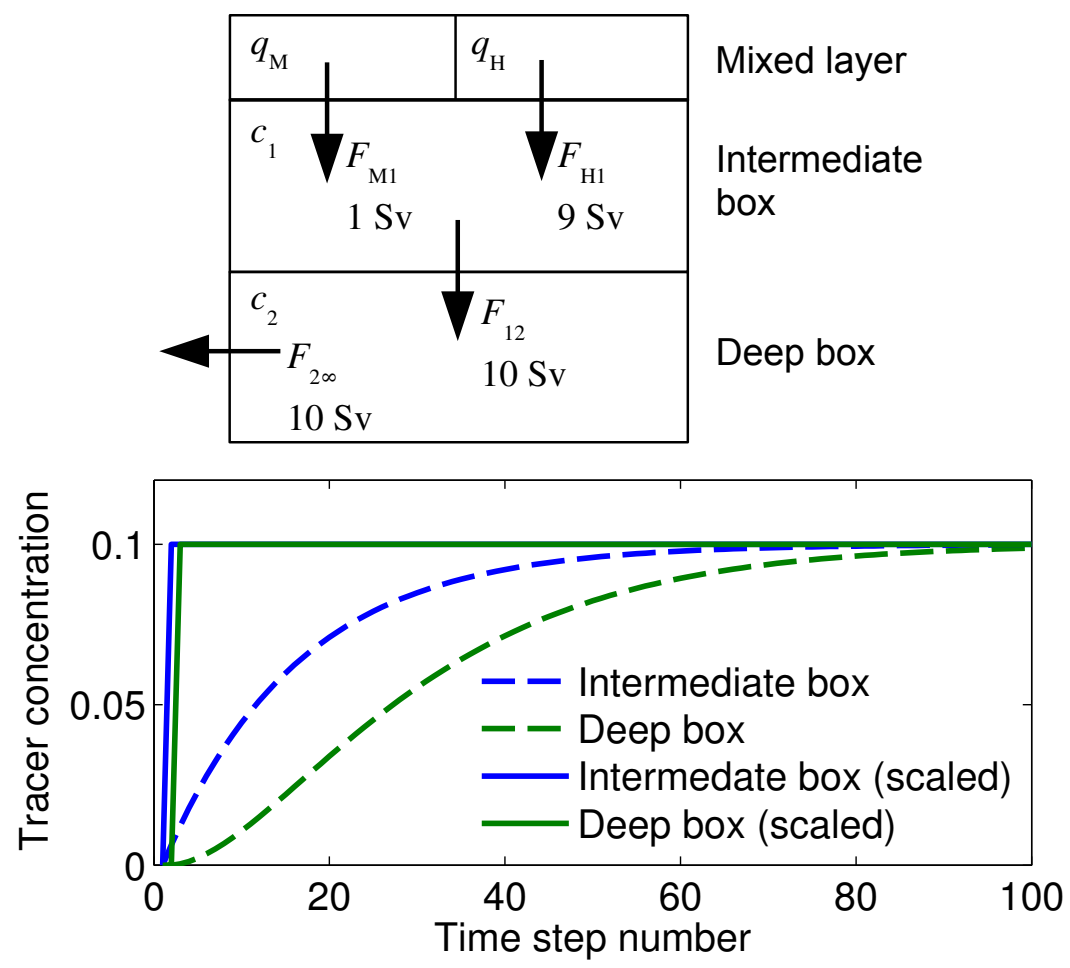

Figure 4.4: TOP: Schematic of the four-box tracer transport model. Passive tracer values $q_{M}$ and $q_{H}$ in two ML boxes are injected into box 1 at rates $F_{M}=1 \mathrm{~Sv}$ and $F_{H}=9 \mathrm{~Sv}$. Fluid is homogenized in box 1 and flows into box 2 at a rate $F_{M}+F_{H}=10 \mathrm{~Sv}$, where it subsequently leaves the system. BOTTOM: Convergence to tracer equilibrium states under boundary conditions $q_{M}=1, q_{H}=0$ under standard (dashed) and preconditioned (solid) integration.

\subsubsection{Extrapolating tracer values using a "helper" tracer}

A complementary approach to the acceleration of tracer advection and diffusion in an OGCM is extrapolation, the approximation of equilibrium tracer values based on information available in a finite-time integration. Wunsch and Heimbach [2008] practiced one form of extrapolation when they linearly extrapolated equilibration times for some regions in the North Pacific. This section presents another such approach that assumes that the fractions of grid boxes ventilated by various regions of the model surface ocean are set relatively early in an integration. The approach can be used in the MITgcm adjoint, as demonstrated in Section 


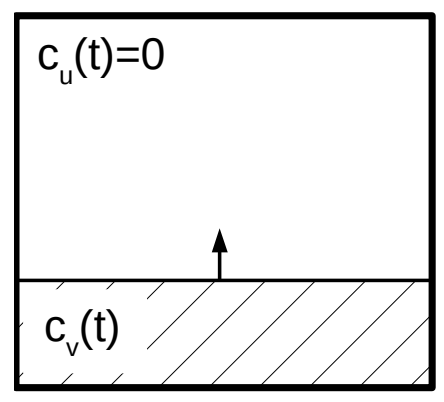

\author{
Unventilated \\ volume $v_{u}(t)$ \\ Ventilated \\ volume $v_{v}(t)$
}

Figure 4.5: Schematic illustrating grid box tracer equilibration as the expansion of the ventilated subvolume. As new waters enter the grid box, the ventilated fraction increases until it occupies the entire grid box at equilibrium.

4.5. making it a useful tool for including equilibrated tracer observations in the cost function.

\title{
4.3.3.1 Development
}

The evolution of tracer values in a grid box as an OGCM is integrated forward in time is typically illustrated by noting the change in grid box concentration, $C(t)$. A complementary approach is to decompose the grid box volume into two fractions, a "ventilated" fraction, $v_{v}(t)$, that has passed through the model mixed layer since the model initiation time $t=t_{0}$, and an "unventilated" fraction, $v_{u}(t)$, that has remained in the interior. As the model steps forward, the ventilated fraction expands and eventually (at $t=\infty$ ) occupies the entire grid box volume $V$, i.e. $v_{v}(\infty)=V$.

This approach solves for the concentration of the ventilated fraction, $c_{v}(t)$, which is a good estimate of $C(\infty)$ in many cases. In the real ocean, we would expect tracer values in many ocean regions never to truly equilibrate to a single value, but instead to have timevariable concentrations affected by seasonal cycles and other variability. However, equilibration to a single constant value is a reasonable approximation at locations where temporal variability is believed to be small, particularly in the deep ocean where most paleoceanographic data lie and where most seasonal variability in tracer transport originating in the 
mixed layer is likely to have been filtered out over long transit times.

The concentration $C(t)$ of an arbitrary grid box can be decomposed as

$$
C(t)=\frac{v_{v}(t) c_{v}(t)+v_{u}(t) c_{u}(t)}{V}
$$

where $c_{u}(t)$ is the concentration of the unventilated volume. If we set $c_{u}=0$ by the choice of initial conditions, the second term is zero and

$$
C(t)=\frac{v_{v}(t)}{V} c_{v}(t) .
$$

As $t \rightarrow \infty, v_{v}(t) \rightarrow V$ and $C(t)$ approaches the equilibrium ventilated tracer value $c_{v}(\infty)$.

If $c_{v}(t)$ is constant for all $t^{\prime}>t$, then $C(\infty)=c_{v}(t)$. Denote $\tilde{C}_{\infty}(t)=c_{v}(t)$ to be the equilibrium value estimated under this assumption. Substituting into Equation (4.35) and rearranging gives

$$
\tilde{C}_{\infty}(t)=\frac{V}{v_{v}(t)} C(t) .
$$

The ratio $V / v_{v}(t)$ of total grid box volume to ventilated volume can be computed at a time $t$ by use of a "helper tracer" that is initialized at zero concentration everywhere and subject to uniform tracer concentration of 1 at all boundary locations. By this choice of boundary conditions, the ventilated concentration of helper tracer is $c_{v}^{h}(t)=1$ at all times, so that Equation 4.35 becomes

$$
C^{h}(t)=\frac{v_{v}(t)}{V} .
$$

Substituting into Equation 4.36 yields

$$
\tilde{C}_{\infty}(t)=\frac{C(t)}{C^{h}(t)},
$$

an estimate of the equilibrium tracer value in the grid box. Because $C^{h}(t) \leq 1, \tilde{C}(t)>C(t)$ 
for all finite $t$. An important property of this estimate is that as $t \rightarrow \infty, C^{h}(t) \rightarrow 1$, so that the estimate converges to the true equilibrium value, $\lim _{t \rightarrow \infty} \tilde{C}_{\infty}(t)=C(\infty)$.

Intuitively, the helper tracer is exactly the fraction of the grid box that has been ventilated. If, for instance, that fraction is $v_{v}(t) / V=C^{h}(t)=0.33$, then dividing by $C^{h}(t)$ is equivalent to multiplying by 3 the amount (in moles or whichever relevant unit) of tracer in the grid box, the same thing that would happen if the rest of the grid box were ventilated and "filled up" with tracer-bearing fluid.

The accuracy of the assumption that $c_{v}(t)$ is constant determines the accuracy of the extrapolation. Regions in the ocean interior can have many time scales of ventilation, e.g. from advective (more rapid) and diffusive (slower) pathways. As such, early in an integration, extrapolated values will be biased towards tracer contributions borne along more rapid pathways. As an integration progresses, $c_{v}(t)$ approaches the constant final value and the assumption becomes increasingly accurate. Knowledge of how $c_{v}(t)$ changes in time, if available, could be used to improve the extrapolation.

Support for the validity of this approximation comes from Amrhein et al. [2015], who showed that over $90 \%$ of the variability in deep ocean adjoint Green's functions (Figure 2.3, derived from the tracer transport model of Gebbie and Huybers [2012]) could be represented using the first leading EOF-PC pairs. Thus, in that model, the fractional ventilating contributions from different surface grid boxes at deep ocean grid boxes could be described to first order by a spatial pattern that was constant, i.e. the spatial distribution of regions ventilating the deep ocean did not appear to be a strong function of time scale. Physical reasons for this observation are not obvious and will be the subject of future investigations.

In practice when used in a numerical model, concentrations of both tracers are very low or zero at the beginning of integration, yielding a singularity in Equation (4.38). This effect 
is mitigated by adding a small value $\varepsilon$ into the denominator and multiplying by $1+\varepsilon$, so that

$$
\tilde{C}_{\infty}(t)=\frac{C(t)(1+\varepsilon)}{C^{h}(t)+\varepsilon}
$$

Multiplication by $1+\varepsilon$ forces convergence of $\tilde{C}_{\infty}(t)$ to $C(t)$ as $t \rightarrow \infty$. The disadvantage of using $\varepsilon>0$ is that estimates for finite $t$ will be biased low.

\subsubsection{Demonstration in a four-box model}

Next we evaluate and demonstrate some properties of the helper tracer approach in a four-box model. The model is very similar to that described in Figure 4.4, except that the high-latitude ML box connects directly to the deep ocean volume, so that there are distinct "slow" (via the intermediate box) and "fast" (directly from the high-latitude mixed layer) tracer transport pathways to the deep box. Fluid with tracer concentration equal to $q_{M}(t)$ and $q_{H}(t)$ is transported at constant rates $F_{M}=1 \mathrm{~Sv}$ and $F_{H}=9 \mathrm{~Sv}$ from middle- and high-latitude ML boxes, respectively, to an intermediate box with volume $V_{1}=10^{8} \mathrm{~km}^{3}$ and tracer concentration $c_{1}(t)$. The intermediate box in turn exchanges fluid at constant rate $F_{12}=10 \mathrm{~Sv}$ with a deep box with volume $V_{2}=10^{8} \mathrm{~km}^{3}$ and tracer concentration $c_{2}(t)$. A compensating flux, $F_{2 \infty}=10 \mathrm{~Sv}$, evacuates fluid from the deep box out of the system.

The state vector in the four-box model is $\mathbf{c}(t)=\left[c_{1}(t), c_{2}(t)\right]^{\top}$ and the ML vector is $\mathbf{q}(t)=\left[q_{M}(t), q_{H}(t)\right]^{\top}$. Combining the equations for conservation of volume and tracer, the evolution in each box is described by a vector equation in the form of Eq. 2.1],

$$
\mathbf{c}(t+\Delta t)=\left[\begin{array}{cc}
1-\frac{F_{M}+F_{H}}{V_{1}} \Delta t & 0 \\
\frac{F_{M}+F_{H}}{V_{2}} \Delta t & 1-\frac{F_{M}+2 F_{H}}{V_{2}} \Delta t
\end{array}\right] \mathbf{c}(t)+\left[\begin{array}{cc}
\frac{F_{M} \Delta t}{V_{1}} & \frac{F_{H} \Delta t}{V_{1}} \\
0 & \frac{F_{H} \Delta t}{V_{2}}
\end{array}\right] \mathbf{q}(t)
$$




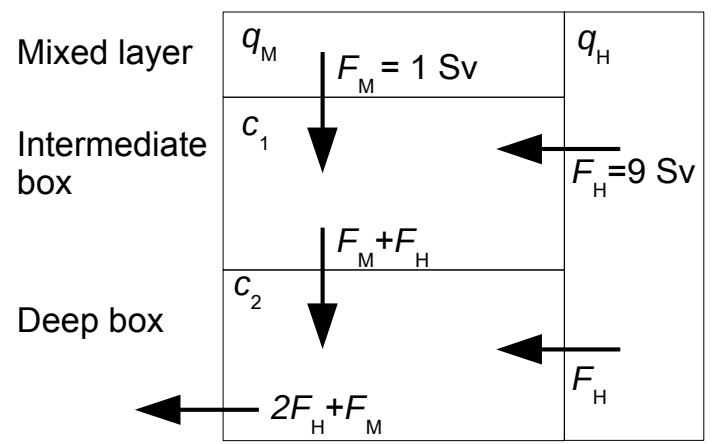

Figure 4.6: Four-box tracer transport model schematic modified from Figure 2.9. Tracer concentration values $q_{M}$ and $q_{H}$ in two mixed-layer boxes are injected into box 1 at rates $F_{M}=1 \mathrm{~Sv}$ and $F_{H}=9 \mathrm{~Sv}$, and $q_{H}$ is injected into box 2 at rate $F_{H}=9 \mathrm{~Sv}$. Fluid is homogenized in box 1 and flows into box 2 at a rate $F_{M}+F_{H}=10 \mathrm{~Sv}$, from which it eventually leaves the system.

Figure 4.7 compares tracer concentrations and extrapolated equilibrium concentrations in the intermediate and deep boxes under initial conditions $\mathbf{c}(0)=[0,0]^{\top}$ and under two different sets of boundary conditions over a 3000 year integration. In the first set, constant boundary conditions $q_{M}=1, q_{H}=0$ are imposed beginning at $t=0$ and in the second case the boundary conditions are $q_{M}=0, q_{H}=1$. In both cases, the intermediate concentration $C_{1}(t)$ gradually relaxes to equilibrium, while extrapolated values $\tilde{C}_{\infty 1}(t)$ take exactly the equilibrium value after the first time step. The intermediate box exemplifies ocean regions connected to the ocean surface via one or more "fast" pathways, whereby the fraction of ventilating water masses is established very early in an integration, and the extrapolation is very accurate almost immediately. By contrast, in the deep box under $q_{M}=0, q_{H}=1$, the approach to equilibrium of $\tilde{C}_{\infty 2}(t)$ is only slightly faster than $C_{2}(t)$ because the ventilated fraction originating from $q_{M}$ is changing as a function of time as box 1 (which feeds box 2) equilibrates. This illustrates the shortcomings of the extrapolation if an ocean region is ventilated by more than one region with very different time scales: because the fraction of ventilating water masses is slowly changing, the extrapolation is only accurate as the box is 

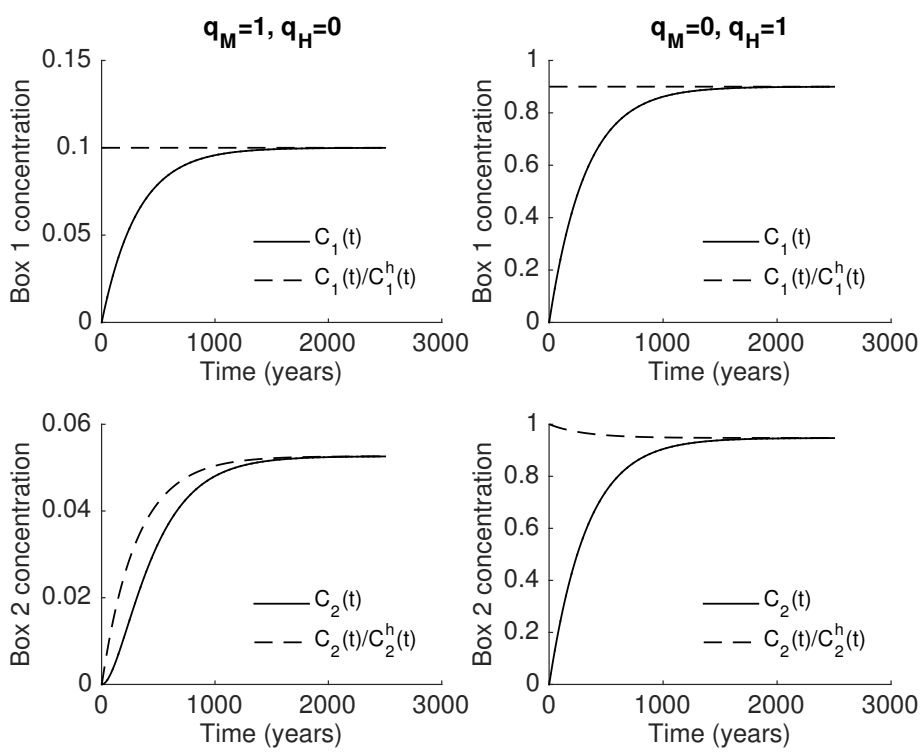

Figure 4.7: Evolution of tracer concentrations (solid lines) and extrapolated equilibrium values (dashed lines) in the intermediate (top) and deep (bottom) boxes of the idealized model in Figure 4.6. The left column corresponds to to surface boundary conditions $q_{M}=1$, $q_{H}=0$ and the right column to $q_{M}=0, q_{H}=1$.

approaching equilibrium. Finally, the deep box under $q_{M}=0, q_{H}=1$ illustrates how the extrapolation may overshoot equilibrium values if tracer values borne along "fast" pathways are larger than those borne by "slow" pathways.

\subsubsection{Extensions and future directions}

This technique can be generalized to the case where tracer initial conditions are not zero, but are nearly constant in time under a previous set of boundary conditions (for instance, after a long period of tracer spin-up). In this case, let $t=0$ be the time when new concentration boundary conditions are imposed, and put $c_{u}=C(0)$. Then, noting that $V=v_{u}+v_{v}$, Equation (4.34) becomes

$$
\begin{aligned}
C(t) & =\frac{v_{v} c_{v}(t)+\left(V-v_{v}\right) C(0)}{V} \\
& =\frac{v_{v}}{V}\left(c_{v}(t)-C(0)\right)+C(0) .
\end{aligned}
$$


Introducing the helper tracer through Equation 4.37) yields

$$
C(t)=C^{h}(t)\left(c_{v}(t)-C(0)\right)+C(0)
$$

Solving for $c_{v}(t)=\tilde{C}(t)$,

$$
\begin{aligned}
c_{v}(t) & =C(0)+\frac{1}{C^{h}(t)}(C(t)-C(0)) \\
& =\frac{C(t)}{C^{h}(t)}-\left(\frac{1-C^{h}(t)}{C^{h}(t)}\right) C(0) \\
\Longrightarrow \tilde{C}_{\infty}(t) & =\frac{C(t)}{C^{h}(t)}-\left(\frac{1-C^{h}(t)}{C^{h}(t)}\right) C(0)
\end{aligned}
$$

The factor multiplying $C(0)$ in the subtracted term is always positive and approaches zero as $t \rightarrow \infty$; it is a correction that subtracts the concentration due to unventilated tracer. As before, it will be necessary to introduce small numbers in the denominators of Equation (4.46) to avoid singularities, so that

$$
\tilde{C}_{\infty}(t)=\frac{C(t)(1+\varepsilon)}{C^{h}(t)+\varepsilon}-\frac{\left(1-C^{h}(t)\right)\left(1+\varepsilon_{1}\right)}{C^{h}(t)+\varepsilon_{1}} C(0)
$$

This generalization is of practical use in the case where a long tracer equilibration already exists and one is interested in equilibrating a boundary condition perturbation.

This approach may be of use in cases where one is interested in estimating the equilibrium passive tracer response to a change in the ocean circulation, e.g., from an instantaneous strengthening of the wind field. If time scales of tracer concentrations due to such circulation changes are similar to those of $C_{h}(t)$ (for instance, if the circulation changes primarily result in a change in tracer concentrations of waters exported to the deep ocean), then the problem is similar to the case with nonzero initial conditions and changing boundary conditions discussed in the previous paragraph. If the circulation changes are more complex 
and drive a rerouting of ocean tracer transport pathways, there may be complicated transient behavior as the new circulation flushes out the initial tracer distribution, and the helper tracer approach could yield unstable results. The scheme is nevertheless guaranteed to converge to true equilibrium values and may have some utility after an initial interval.

\subsection{Evaluating tracer equilibration procedures in the MIT- gcm}

Here we test the procedures developed in Section 4.3 in the context of a full OGCM. Extrapolated tracer values were computed with and without vertically varying tracer integration time steps in a 3700-year integration of the MITgcm. The model is an evolved form of that described by Marshall et al. [1997] and Adcroft et al. [2004] and simulates the ocean circulation under hydrostatic and Boussinesq assumptions. The model domain [Lauderdale et al., 2013] is global with 2.8 degree horizontal resolution and 15 non-uniform vertical levels. The model is forced by 12-month cycles of heat and freshwater fluxes with additional relaxation toward seasonal cycles of temperature and salinity with time scales of 60 and 90 days, respectively. Monthly winds are from the ECMWF annual climatology [Trenberth et al., 1989]. Transport by mesoscale eddies is parameterized using the Gent and McWilliams [1990] scheme with constant isopycnal thickness diffusivity of $10^{3} \mathrm{~m}^{2} \mathrm{~s}^{-1}$ and vertical mixing by a diapycnal diffusivity of $5 \times 10^{-5} \mathrm{~m}^{2} \mathrm{~s}^{-1}$. Prior to integrating the passive tracer, the model was spun up for 25,000 years to a nearly seasonally steady state. Boundary conditions used (Figure 4.9) are from an estimate of the modern distribution of preformed oxygen, which is treated in the model as a passive, conservative tracer.

To generate vertically varying time step values, I first computed the maximum value $m_{k}$ 


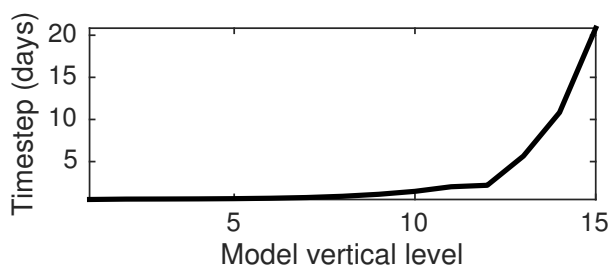

Figure 4.8: Passive tracer time step as a function of vertical level in the 15-layer model used for forward tracer simulations.

of the mass-weighted velocities within each vertical layer,

$$
m_{k}=\max _{i, j}\left(\left|\mathrm{UVELMASS}_{i, j, k}\right|+\left|\operatorname{VVELMASS}_{i, j, k}\right|+\left|\mathrm{WVELMASS}_{i, j, k}\right|\right)
$$

where $\mathrm{UVELMASS}_{i, j, k}, \mathrm{VVELMASS}_{i, j, k}$ and $\mathrm{WVELMASS}_{i, j, k}$ are the meridional, zonal, and vertical mass-weighted velocities at the model grid box indexed by $i, j, k$ in the last year of a control simulation. The depth-varying time step, $t_{k}$, was computed as

$$
t_{k}=t_{1}+\left(\frac{m_{1}}{m_{k}} t_{1}-t_{1}\right) / 2
$$

where $t_{1}$ is the time step in the top most grid box (here, 12 hours). This choice is tantamount to using the approach documented in Section 4.3.2 on a per-level basis assuming that the top layer is at the CFL limit, ignoring changes in grid box volume as a function of depth, and dividing the changes in depth by a factor of 2 . This scheme was chosen because it was found through trial and error to be numerically stable. Further experiments can likely increase the time steps as a function of depth even more, with commensurate acceleration to tracer equilibrium (albeit at the risk of tracer nonconservation; see Danabasoglu [2004]).

Figure 4.10 compares time series of tracer concentrations computed with and without vertically varying tracer time steps, $C^{v}(t)$ and $C(t)$, and corresponding equilibrium estimates $\tilde{C}_{\infty}^{v}(t)$ and $\tilde{C}_{\infty}(t)$ obtained from model grid box locations in the North Atlantic, North Pacific, and Southern Oceans at various depths. The value of $\varepsilon=0.05$ in Equation (4.39) 


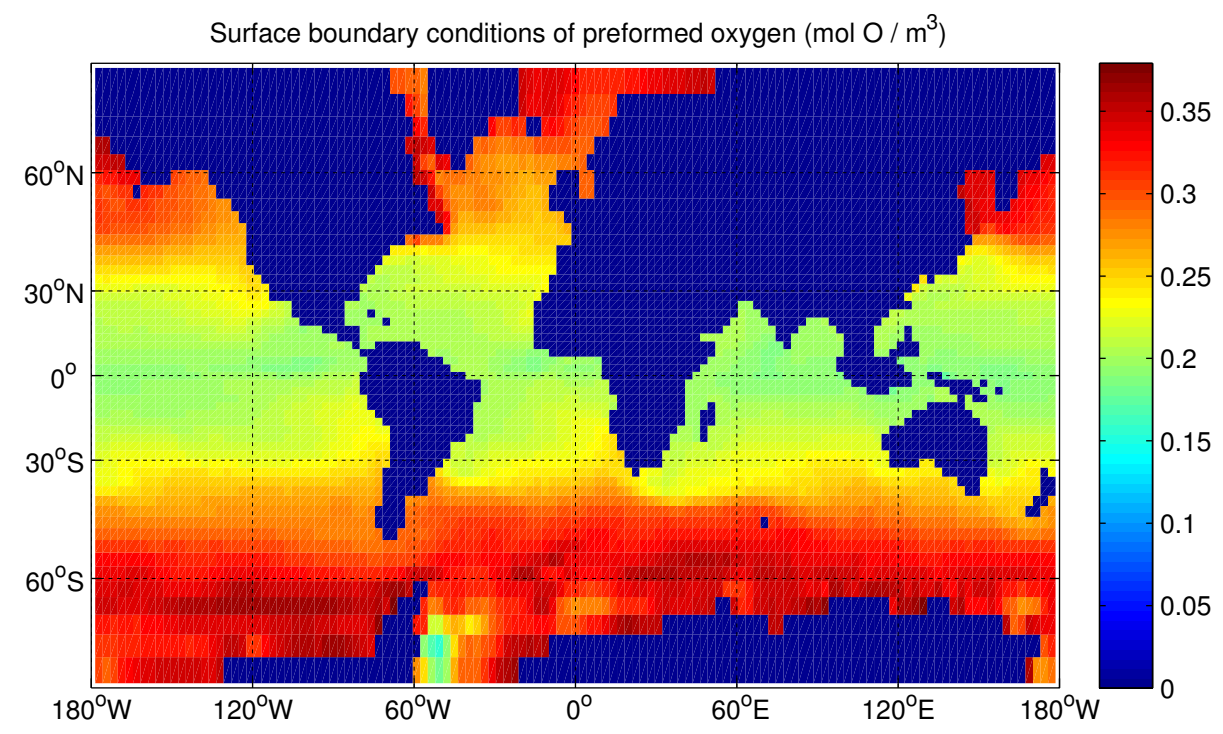

Figure 4.9: Constant (in time) surface concentration boundary conditions of preformed oxygen in $\mathrm{mol} \mathrm{O} / \mathrm{m}^{3}$. Values do not exceed 0.4 .

was chosen empirically; the choice of $10^{-3}$ yielded faster convergence, at the expense of overshoots in more locations, and the choice of $10^{-1}$ eliminated some numerical artifacts but yielded much slower convergence. At all locations, concentration and extrapolated concentrations appear to converge to the same final value. Use of extrapolation and vertical time step variation uniformly accelerates estimation of equilibria, and $\tilde{C}_{\infty}^{v}(t)$ is nearest the respective equilibrium values at all locations and times. The difference is most notable in the North Pacific, where tracer transit times are generally longest (Wunsch and Heimbach, 2008) and where $90 \%$ equilibrium times of $\tilde{C}_{\infty}^{v}(t)$ are less than a tenth of the time than for $C(t)$.

The location in the Southern Ocean was deliberately chosen to demonstrate the effects of numerical artifacts on $\tilde{C}_{\infty}(t)$. In the first 100 years, equilibrium estimates there are as high as $1200 \mathrm{~mol} \mathrm{O} \mathrm{m} \mathrm{m}^{-1}$. After 100-200 years these values return to smooth trajectories as seen at the other grid boxes. Increasing $\varepsilon$ to $10^{-1}$ eliminated these artifacts at the expense of slower convergence rates. To assess the spatial distribution of grid boxes affected by this artifact, the top panel of Figure 4.10 shows the map of maximum values of $\tilde{C}(t)$ at all depths 

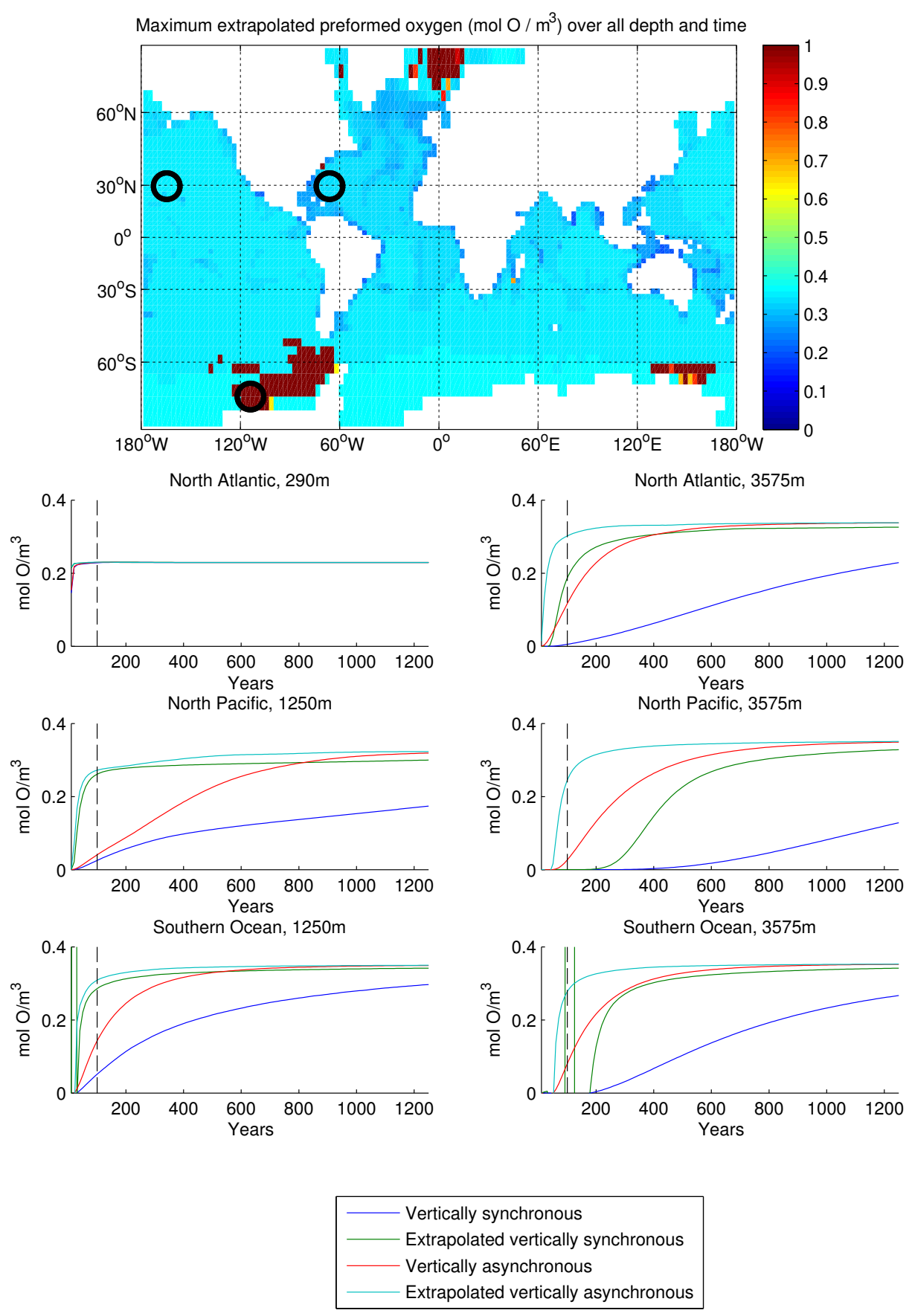

Figure 4.10: Top: Maximum values of $\tilde{C}_{\infty}(t)$ over all depths and times. Locations where values are larger than roughly $0.4 \mathrm{~mol} \mathrm{O} \mathrm{m}{ }^{-1}$ are affected by numerical artifacts. Bottom: Comparison at different locations of preformed oxygen concentrations, $C(t)$, and equilibrium estimates, $\tilde{C}_{\infty}(t)$, for vertically synchronous and asynchronous time steps computed over a 1250 year MITgcm simulation using $\varepsilon=0.05$. Grid box locations are given by black circles in the top panel. Large positive excursions in the Southern Ocean estimates arise from numerical artifacts. Extrapolated, vertically asynchronous estimates converge most quickly at all locations. Dotted vertical lines lie at 100 years, the duration integration used in Section 4.5 . 
and times. Values higher than $0.4 \mathrm{~mol} \mathrm{O} \mathrm{m}^{-1}$ (the maximum of the imposed concentration boundary conditions) indicate locations affected by numerical artifacts. The distribution of these locations reflects model regions where deep water forms via parameterized convection. Implicit vertical tracer diffusion, which acts more strongly on tracers with larger gradients, could be to blame, as could negative tracer values in the helper tracer driven by horizontal eddy parameterizations that could make the denominator in Equation (4.39) very small.

Equilibration times for active tracers (such as temperature and salinity) or nonconservative tracers (such as nutrients) could be accelerated in some regimes where the tracers behave nearly passively or where equilibration times are similar to those of $C_{h}(t)$. To evaluate this possibility I considered the ideal age tracer, which has zero surface boundary conditions and whose evolution in the interior is governed by the advective-diffusive equation with a source term,

$$
\frac{\partial A}{\partial t}=\mathbf{u} \cdot \nabla A-\nabla \cdot(\kappa \nabla A)+1
$$

where $A$ has units of time, $\mathbf{u}$ is the model velocity vector, and $\kappa$ is the mixing tensor.

Figure 4.11 shows the extrapolation applied to an ideal age tracer simulated in the same forward model run described above without vertically varying time steps. Extrapolated values yield improvements in convergence at some times and some locations, though with substantial overshoots that persist for thousands of model years. Unlike in the passive tracer case, $\tilde{C}_{\infty}(t)$ converges to $C(\infty)$ from above in some cases; this is because grid boxes that have low fractions of ventilated water in the spin-up will appear much older than they are at equilibrium. The pattern of maximum values in time and space does not have large values in deep water formation regions as seen in Figure 4.10, possibly because the vertical gradients between $C(t)$ and $C^{h}(t)$ in this case (which drive tracer transport through implicit diffusion) are more similar than for preformed oxygen. Instead, very high $(>30,000$ years) extrapolated ideal ages are computed for regions in the North Pacific even after 500 model years; 
in that region, ideal ages are expected to be oldest and may be inflated by small values in the denominator of Equation (4.39). Further efforts are necessary to determine whether a different scaling using $C^{h}(t)$ or a different type of helper tracer is better suited to the ideal age and other nonconservative tracers. 


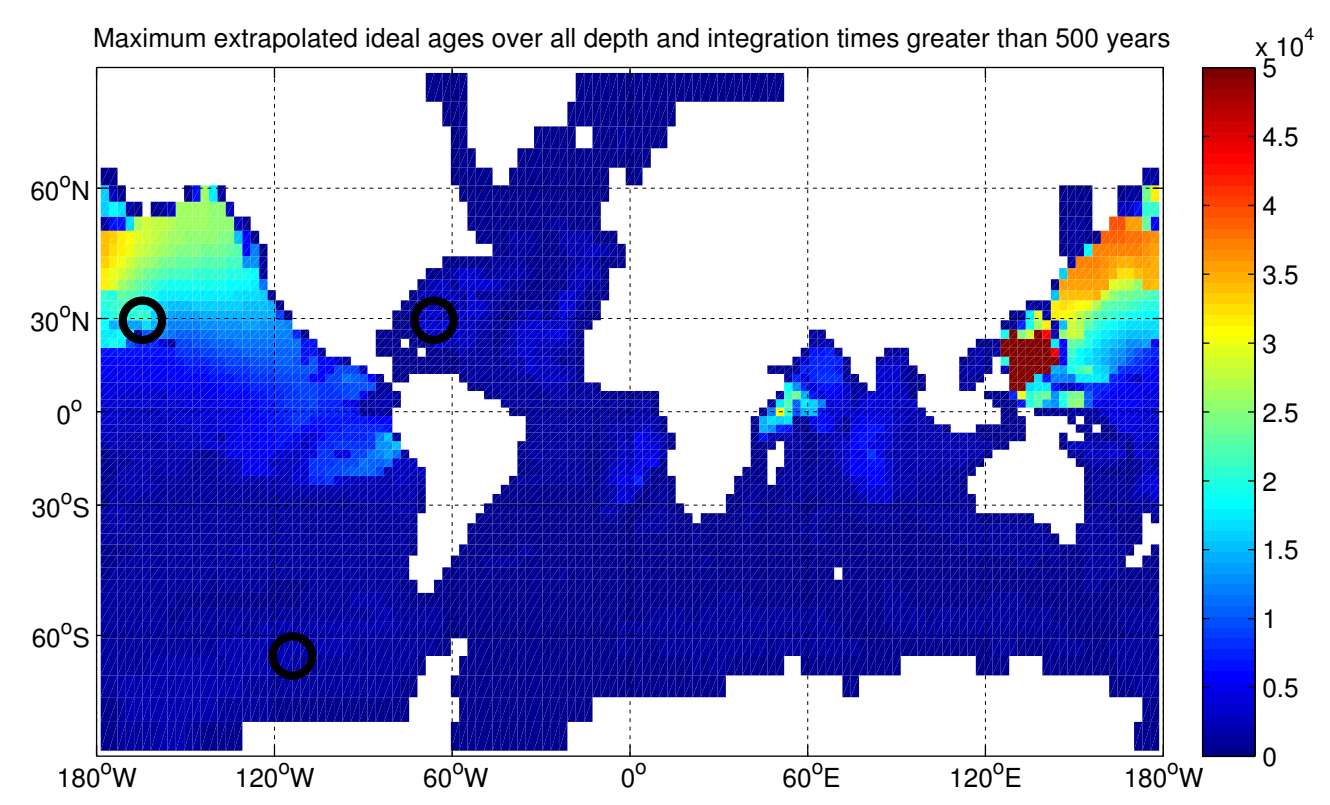

North Atlantic, $1250 \mathrm{~m}$

North Atlantic, 3575m
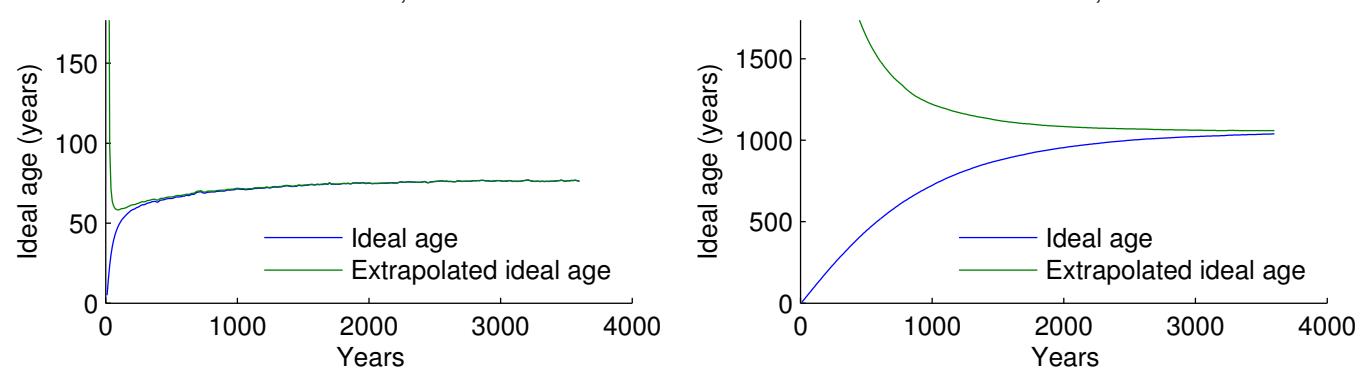

North Pacific, 1250m
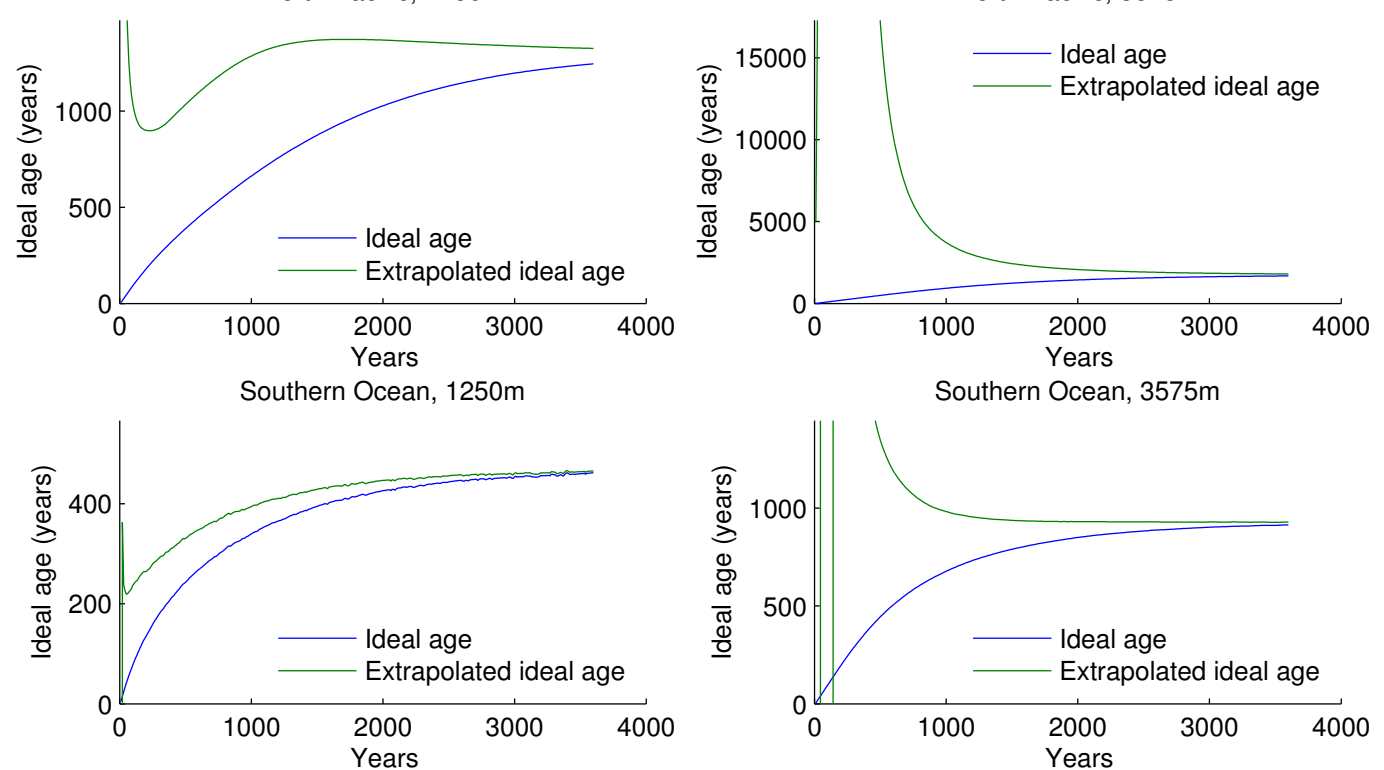

Figure 4.11: Same as Figure 4.10 but for ideal age and only computed using vertically synchronous time stepping. In the top panel the maximum values of $\tilde{C}(t)$ have been computed over all depths and times greater than 500 years due to the prevalence of overshoots during initial centuries of simulation. 


\subsection{Why might Antarctic Bottom Water shoal in the At- lantic Ocean?}

\subsubsection{Introduction}

This section applies the procedures described in previous sections to the problem of inferring ocean circulation changes from passive tracer observations. The motivation for this hypothetical experiment is the hypothesis that the LGM ocean was filled dominantly by Antarctic Bottom Water (AABW), with a lesser role for North Atlantic Deep Water (NADW). Observations interpreted to support this hypothesis have arisen from a variety of tracer types [Boyle and Keigwin, 1982, Curry and Lohmann, 1982, Oppo and Lehman, 1993, Lund et al., 2011, Marchitto et al., 2002, Marchitto and Broecker, 2006], and possible reasons for these changes include changes in the rate of Atlantic overturning circulation [Curry and Oppo, 2005] and nonconservative tracer effects [Gebbie, 2014]. The subject has broader implications for the climate system, as a larger reservoir of nutrient-rich southern-source waters could explain glacial-interglacial changes in carbon dioxide [Brovkin et al., 2007]. In addition to a basic test of using estimates of equilibrated passive tracers in the cost function, this choice of hy-

pothetical data is designed to test whether the model can adjust to accommodate the kinds of watermass distribution changes that one might expect to see at the LGM, with implications for interpreting results in Chapter 3 .

\subsubsection{Methods}

\subsubsection{MITgem configuration}

As in Section 4.4, the OGCM used is the MITgcm, with model bathymetry, atmospheric boundary conditions, internal mixing parameters, and initial conditions all from the ECCO v4 solution downsampled to a horizontal grid with half the resolution (llc45, nominally 2 degrees with higher resolution at the equator and poles) and the same number of vertical 


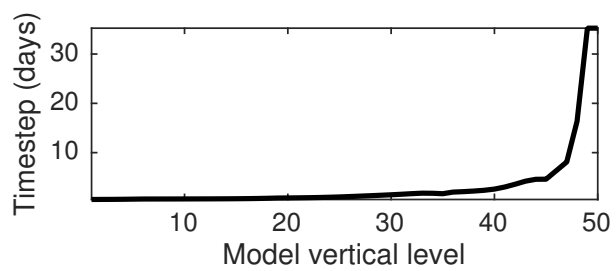

Figure 4.12: Passive tracer time step as a function of vertical level in the 50-layer model used for adjoint tracer simulations.

levels (50). An integration time of 100 years was chosen to minimize computational time and to yield sufficiently equilibrated extrapolated tracer values in much of the Atlantic in order to permit a basic test of the methods.

Two forms of asynchronous time stepping are used. The momentum time step is equal to 20 minutes and the tracer time step is 12 hours for temperature and salinity at all vertical levels. In addition, vertically asynchronous time stepping is employed for passive tracers, with a time step of 12 hours at the surface grid box and 1 month in the deepest grid box (Figure 4.12). Model transports over a 100-year simulation are highest in the initial transient as the model velocity fields (which are initialized to 0 ) adjust to the initial density field and atmospheric forcing. Using large passive tracer time steps computed per Equation (4.49) using the last time step of a control simulation yields numerical instabilities in the passive tracer fields during this initial transient. For this reason, all passive tracer boundary conditions are imposed beginning after the first year of model integration. In the extrapolation procedure, $\varepsilon=0.05$ was chosen by trial and error; $\varepsilon=0.01$ was found to generate instability in the adjoint simulation, believed to originate from division by very small numbers in Equation 4.39 because of negative tracer values generated by numerical artifacts.

\subsubsection{Setup of the inverse problem}

Control variables used (Table 4.1) and their first guess control values and prior standard deviations are taken from the ECCO v4 setup. Passive tracer initial and boundary conditions 
are not included in the control vector, in contrast with Dail [2012b], forcing the model to fit tracer observations using only the model dynamics. Control uncertainties $(\sigma)$ listed in Table 4.1 are the square roots of diagonal values of the control prior covariance matrices, $\mathbf{Q}=\mathbf{I} \sigma^{2}$, in Equation (3.5).

The cost function has contributions penalizing deviations from first-guess controls, two terms penalizing model drift, and a final term due to the misfit between model tracer and the hypothetical observations (Table 4.2). Similar to the controls, the data uncertainty covariance $\mathbf{R}(t)$ (Equation 3.3) is specified as a diagonal matrix. The vertical profile of hypothetical observations is illustrated in Figure 4.13 (bottom panel); deviations from these values are penalized at all vertical levels within the red region in Figure 4.13 (top panel). Tracer misfits are computed only in the last model year, when the extrapolated tracer distribution is closest to true equilibrium. The drift terms are calculated as the sum of the squared deviations of every model year annual average from the 100-year average and serve to minimize longerthan-annual variability in temperature and salinity. Including the drift terms attempts both to generate a seasonally quasi-steady solution and to ensure that the fit to tracer data is not the result of a transient near the end of the simulation.

In order to differentiate deep water masses originating in the Southern Hemisphere and permit their inclusion in the cost function, concentration boundary conditions of 0 north of $60^{\circ} \mathrm{S}$ and 1 south of $60^{\circ} \mathrm{S}$ are enforced for tracer $C^{A A B W}$ by relaxing the top three grid boxes with e-folding time scale equal to the tracer time step (12 hours) after the first model year. Concentration boundary conditions for the helper tracer $C^{h}$ are imposed in the same way, except that they are everywhere 1 in the top three grid boxes. Tracer initial conditions for both tracers are everywhere zero.

Figure 4.13 compares the distribution of extrapolated $\tilde{C}^{A A B W}$ in the initial model simulation (prior to any control adjustments, $\tilde{C}_{\infty, 0}^{A A B W}$ ) after 100 years and the modern distribution of AABW estimated in a watermass census [Johnson, 2008]. Modeled $\tilde{C}_{\infty, 0}^{A A B W}$ is qualitatively 


\begin{tabular}{|c|c|c|c|}
\hline Control variable & $\sigma$ & First guess & Period/duration \\
\hline \hline SW radiation & $20 \mathrm{Wm}^{-2}$ & ECCO v4, 2007 & Monthly climatology \\
\hline LW radiation & $20 \mathrm{Wm}^{-2}$ & ECCO v4, 2007 & Monthly climatology \\
\hline Humidity & $2 \times 10^{-3}$ & ECCO v4, 2007 & Monthly climatology \\
\hline Precipitation & $4 \times 10^{-8}$ & ECCO v4, 2007 & Monthly climatology \\
\hline $2 \mathrm{~m}$ air temperature & $4^{\circ} \mathrm{K}$ & ECCO v4, 2007 & Monthly climatology \\
\hline Zonal wind stress & $1 \mathrm{~Pa}$ & ECCO v4, 2007 & Monthly climatology \\
\hline Meridional wind stress & $1 \mathrm{~Pa}$ & ECCO v4, 2007 & Monthly climatology \\
\hline$\kappa_{d}$ & $10^{-4} \mathrm{~m}^{2} \mathrm{~s}^{-1}$ & ECCO v4 & Constant \\
\hline$\kappa_{\sigma}$ & $500 \mathrm{~m}^{2} \mathrm{~s}^{-1}$ & ECCO v4 & Constant \\
\hline$\kappa_{G M}$ & $500 \mathrm{~m}^{2} \mathrm{~s}^{-1}$ & ECCO v4 & Constant \\
\hline Initial temperature & $3^{\circ} \mathrm{K}$ & ECCO v4, Jan 1, 2007 & Initial \\
\hline Initial salinity & $1 \mathrm{~g} \mathrm{~kg}^{-1}$ & ECCO v4, Jan 1,2007 & Initial \\
\hline
\end{tabular}

Table 4.1: Control variables, first guesses, prior control adjustment uncertainties $(\sigma)$, smoothing extents, and period and duration for control elements used in the idealized adjoint simulation. All values are taken from the ECCO v4 simulation in the year 2007, and averaged in space to be used on the lower-resolution grid. $\kappa_{\sigma}, \kappa_{d}$, and $\kappa_{G M}$ refer to coefficients of isopycnal diffusivity [Redi, 1982], diapycnal diffusivity, and eddy bolus velocity [Gent and McWilliams, 1990].

\begin{tabular}{|c|c|c|}
\hline Cost function term & $\sigma$ & Period/Duration \\
\hline \hline Deviation from first-guess controls & see Table 4.1 & Various/All years \\
\hline Misfit of extrapolated tracer to bogus tracer data & $0.2 \mathrm{~mol} / \mathrm{L}$ & Annual average/Year 100 \\
\hline Deviation of annual-mean S from 100-yr mean & $0.1 \mathrm{~g} / \mathrm{km}$ & Annual average/All years \\
\hline Deviation of annual-mean T from 100-yr mean & $0.1 \mathrm{~K}$ & Annual average/All years \\
\hline
\end{tabular}

Table 4.2: Contributions to the the cost function in the idealized adjoint simulation. 
similar to the modern estimate in many respects. In the Southern Ocean, $\tilde{C}_{\infty, 0}^{A A B W}$ increases with depth below 1000 meters, suggesting that the tracer tracks a dense watermass communicated to the abyss by parameterized convection or salt plume processes. The northward penetration of $\tilde{C}_{\infty, 0}^{A A B W}$ in the deepest part of the western Atlantic is smaller than in the census, possibly because $\tilde{C}_{\infty, 0}^{A A B W}$ is still biased low after 100 years in the deeper reaches of the Atlantic. Greater penetration of sub-thermocline $\tilde{C}_{\infty, 0}^{A A B W}$ (Antarctic Intermediate Waters) could be explained by differences in watermass labeling (dye release versus T/S property analysis used in Johnson [2008]). Model errors likely also contribute to these misfits; the representation of the abyssal circulation in ECCO v4 is constrained by relatively few measurements

(compared to the upper ocean), particularly in the Southern Ocean. Overall the $\tilde{C}_{\infty, 0}^{A A B W}$ distribution appears to be a plausible starting place to ask questions about sensitivity of the model AABW-NADW boundary.

The bottom panel of Figure 4.13 shows the vertical profile of artificial observations, which indicate a greatly enhanced presence of AABW in the Atlantic abyss. Data are 1 below 4200 meters and decrease to 0.2 at 3000 meters, above which they are zero.

\subsubsection{Results}

A first test of passive tracers in state estimation is whether the model parameter adjustments computed in first iteration are able to reduce the model-data misfit. If this is the case, the indication is that useful information about the cost gradient is being generated and used by the model. In a linear optimization problem, sensitivities computed at the first iteration are sufficient to solve the problem and arrive immediately at an optimized solution. In nonlinear optimization problems (such as ocean state estimation), subsequent iterations compute sensitivities for the adjusted model state, and accumulated sensitivities (including interactions between control variable adjustments) may implicate more processes and provide a more 

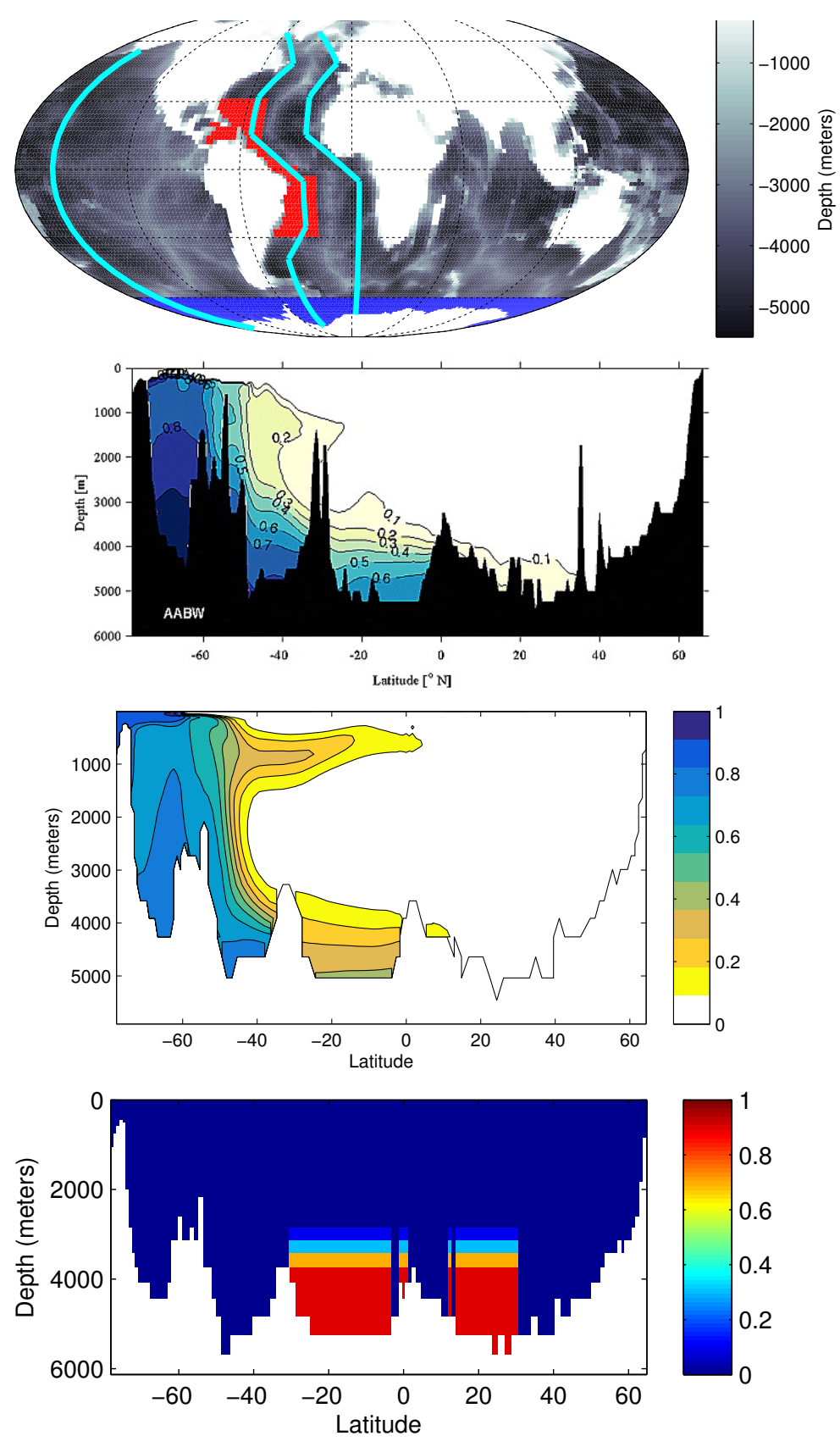

Figure 4.13: Top: Regions of nonzero tracer boundary conditions (blue), hypothetical data (red), and plotted sections (cyan lines) overlaid on model bathymetry used in the idealized adjoint experiment. Second: Fraction of AABW computed by Johnson [2008] along a Western Atlantic section similar to that shown in the top panel. Third: Western Atlantic section of extrapolated passive tracer concentrations after 100 years of integration before any control adjustments are made. Bottom: Western Atlantic section showing the vertical distribution of hypothetical data. 
complete picture of how data may be fit. The remainder of this section analyzes the results from a single iteration and may be viewed as a linear sensitivity experiment. Because a large fraction of model-data misfit sensitivity is often linear in ocean state estimation (P. Heimbach, personal communication), the linear sensitivity is a useful quantity to consider, and benefits from relative ease of interpretation.

Figure 4.14 compares $\tilde{C}_{\infty}^{A A B W}$ between the initial simulation and after making control adjustments computed in the first iteration $\left(\tilde{C}_{\infty, 1}^{A A B W}\right)$ along sections from the Western Atlantic, Eastern Atlantic, and Pacific Oceans (cyan lines, Figure 4.13, top panel). In the first iteration, total cost was reduced by $26 \%$. Anomalies $\tilde{C}_{\infty, 0}^{A A B W}-\tilde{C}_{\infty, 0}^{A A B W}$ in the Western Atlantic show clear increases in $\tilde{C}_{\infty}^{A A B W}$ below 3000 meters between -40 and 20 degrees $\mathrm{N}$, with modest increases further north and small reductions elsewhere in the section. This increase reflects the distribution of the artificial data, and shows that the adjoint sensitivities computed from extrapolated tracer values are able to improve the fit of those extrapolated values to observations. A profile from the Eastern Atlantic, which is largely separated from the Western Atlantic by mid-ocean ridges with model depths of 3000-3500 meters, is qualitatively similar, though the positive anomaly is strongest between 30 and 40 degrees N. Changes along a Pacific section are relatively modest, with a small reduction at depth and a moderate increase centered at roughly 2500 meters.

The improved fit to the hypothetical data owes largely to changes in the turbulent transport parameters $\kappa_{\sigma}, \kappa_{d}$, and especially $\kappa_{G M}$. Figure 4.15 shows the model-data misfit in a forward simulation where only the control adjustments to $\kappa_{G M}$ were used; this simulation recovers most of the improvement in model-data misfit. This parameter scales the parameterized eddy bolus transport in the scheme of Gent and McWilliams [1990] by inducing smaller or larger transports as a function of the zonal and meridional background density stratification, $S_{x}$ and $S_{y}$, 


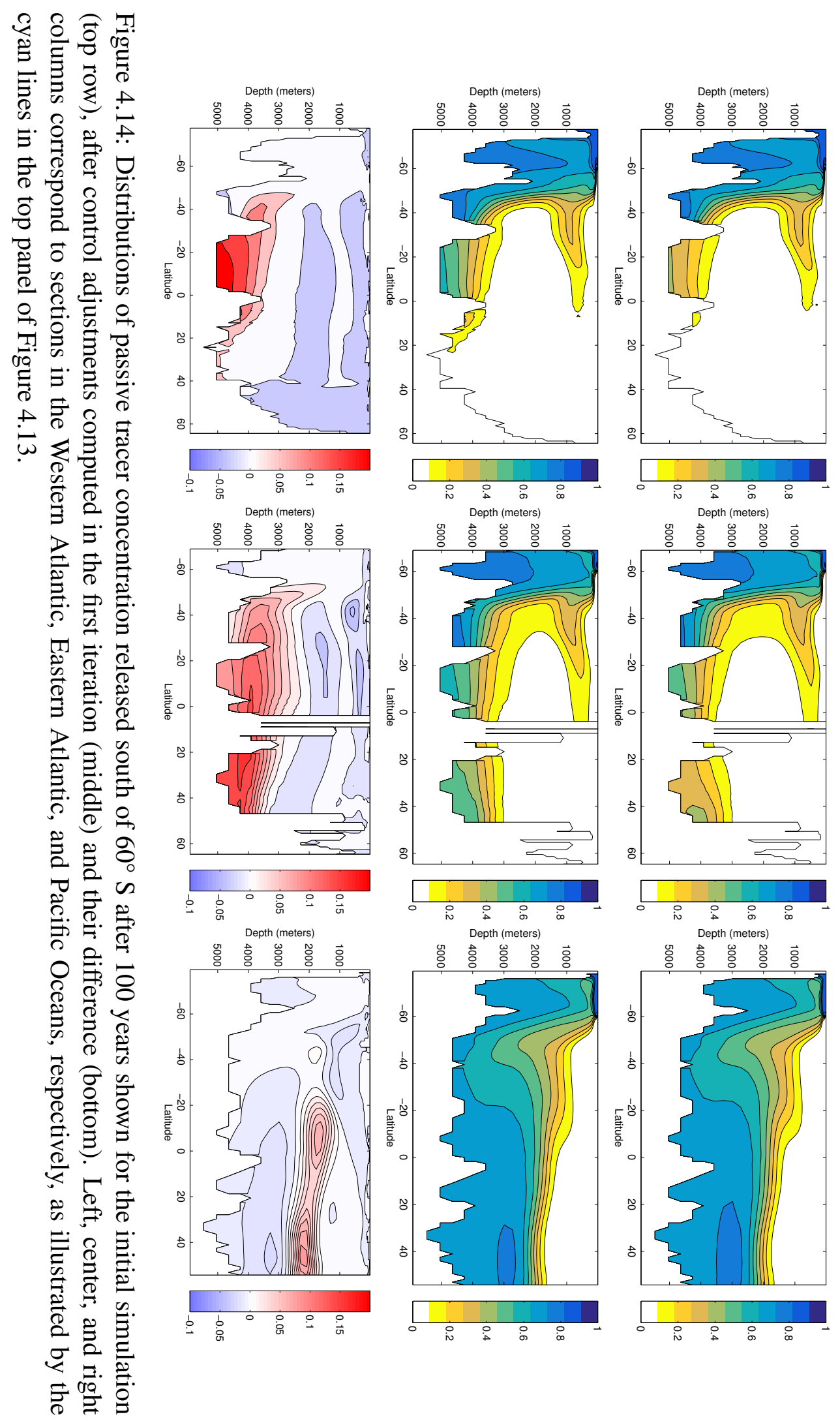




$$
\begin{aligned}
\mathbf{u}^{\star} & =-\nabla \times \Psi^{\star} \\
\Psi^{\star} & =\left(\kappa_{G M} S_{y}, \kappa_{G M} S_{x}\right) .
\end{aligned}
$$

where $\mathbf{u}^{\star}$ and $\Psi^{\star}$ are the bolus velocity and streamfunction, respectively. Patterns of changes in $\kappa_{G M}$ derived in the first iteration (Figure 4.15) are spatially nonuniform and mostly occur in regions of large background tracer gradients and above bathymetric features in the Atlantic. Changes are up to or exceed $500 \mathrm{~m}^{2} \mathrm{~s}^{-1}$ in some regions, in keeping with the prior uncertainty $\sigma_{\kappa_{G M}}=500 \mathrm{~m}^{2} \mathrm{~s}^{-1}$ (Table 4.1 .

To evaluate linear sensitivities when changes in turbulent transport parameters are more restricted, a second experiment was conducted using $\sigma_{\kappa_{\sigma}}=\sigma_{\kappa_{G M}}=50 \mathrm{~m}^{2} \mathrm{~s}^{-1}, \sigma_{\kappa_{d}}=10^{-5} \mathrm{~m}^{2} \mathrm{~s}^{-1}$, corresponding to prior uncertainty values that are ten times smaller than those parameters. In this experiment, changes to wind stresses play a dominant role in improving the fit to data. If only the wind stress changes are added to the first guess forcings and the model is run forward for 100 years, the result is similar to the case with $\kappa_{G M}$ changes, with a marked shoaling of $C^{A A B W}$ in both the Western and Eastern Atlantic (Figure 4.16). Wind stress patterns derived to shoal the tracer boundary are strongest in the Atlantic Equatorial regions; zonal wind anomalies form a dipole across the Equator that is suggestive of a northward shift in the trade winds. One result of these wind stress perturbations is a reduction in model Deep Western Boundary Current velocities centered at $3700 \mathrm{~m}$; this reduction is expected to slow the export of NADW, leading to a larger fraction of model AABW in the Atlantic abyss. The mechanisms by which midlatitude wind changes might alter abyssal flows are various and implicate many flow regimes; future work will describe these mechanisms and whether they might be related to hypothesized changes in the intertropical convergence zone at the LGM (e.g., McGee et al. [2014]). That the wind changes are largest by far near the 

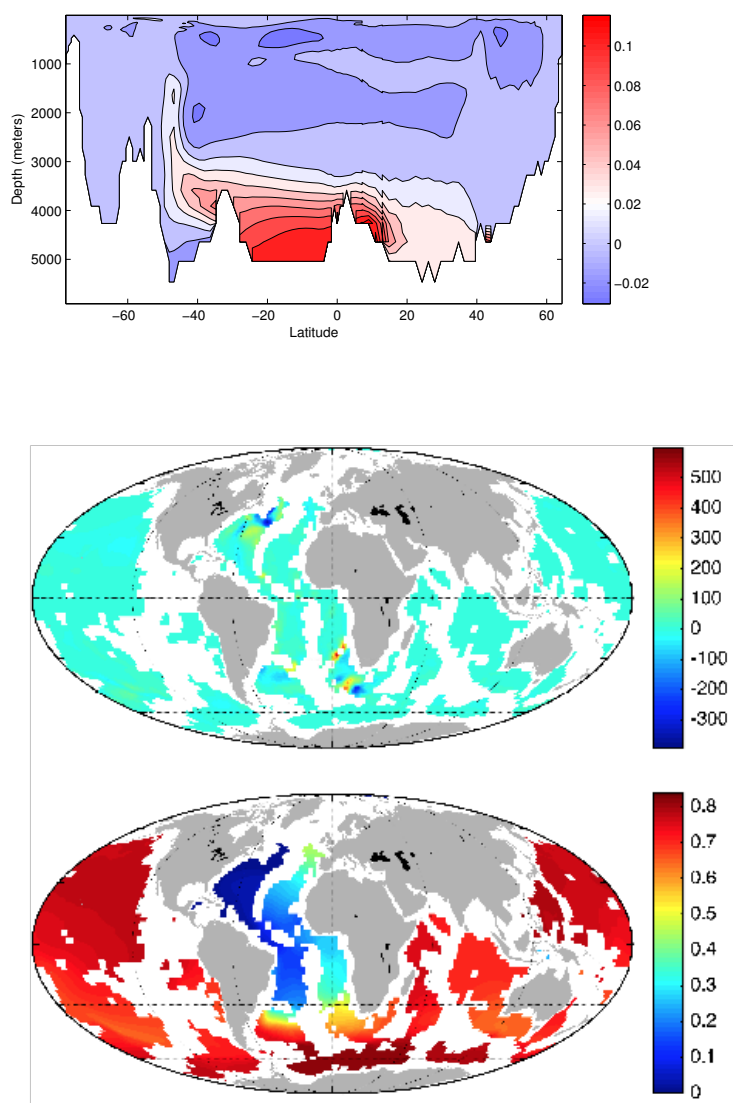

Figure 4.15: Top: Differences in tracer concentrations along the Western Atlantic section between the initial simulation and a forward simulation using only the control changes derived for $\kappa_{G M}$. Most of the shoaling of AABW seen when all controls are used is captured by changing only this variable. Middle: Changes in $\kappa_{G M}$ derived in the first iteration of the idealized tracer inverse experiment. Units are $\mathrm{m}^{2} \mathrm{~s}^{-1}$. Bottom: $C_{\infty, 0}^{A A B W}$ in the 100th year of simulation at $3900 \mathrm{~m}$. Most changes in $\kappa_{G M}$ lie in regions of large background tracer gradients. 
equator may emerge in part from a bias because those regions have adjustment time scales (e.g., by wave propagation) that are much shorter than at high latitudes, and thus those regions can have a stronger effect on fitting the data in this 100-year run than changes at high latitudes, which can take centuries to equilibrate. It is possible that poor representations of eddy compensation, which can negate wind-driven circulation changes, may bias the model towards large sensitivities to wind stress.

\subsubsection{Discussion}

This section demonstrates reductions in the misfit between an estimate of the model equilibrium tracer values and observed tracer distributions via model parameter adjustments derived using the MITgcm adjoint. The approach of using synthetic data can conceivably be extended to other regions, scenarios, and time periods to test what model mechanisms might reproduce hypothesized tracer distributions, and what the effects might be on the rest of the ocean. Such an approach is particularly useful when the evidence for an observed tracer distribution is circumstantial or the result of proxy systems (such as nutrient tracers, which rely on simulating ocean biogeochemical processes and ecosystems) that are very difficult to represent.

The fact that observations of a shoaled tracer boundary in the Western Atlantic are fit by the model by circulation changes that shoal the boundary throughout the Eastern Atlantic as well is promising for the ability of these data to "speak remotely," i.e. to constrain nonlocal physics in the ocean. These results also suggest that there is some synchrony in the movements of the model AABW-NADW boundary in the Western and Eastern Atlantic, as is often assumed in paleoceanographic studies, though there are substantial east-west gradients in equilibrium tracer, particularly at high latitudes. It is not clear to what extent this observation applies to the real ocean where the dominant mechanisms for changing watermass boundaries may be different from those at work in these sensitivity experiments. 

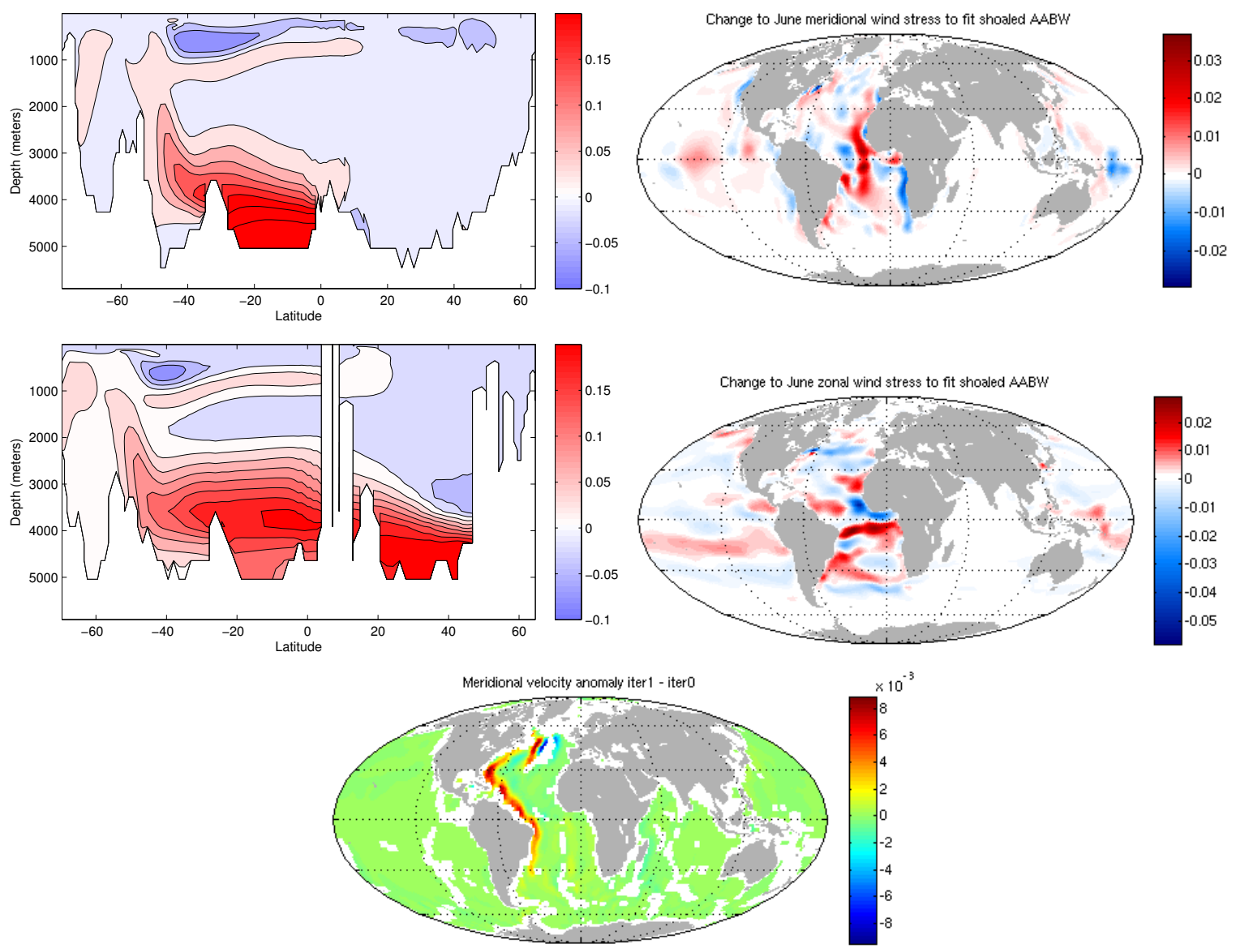

Figure 4.16: Left: Differences in tracer concentrations along the Western (top) and Eastern (bottom) Atlantic sections between the initial simulation and a forward simulation using only the wind stress control changes derived to reduce model misfit to hypothetical $C^{A A B W}$ in the sensitivity experiment with decreased $\sigma_{\kappa_{\sigma}}, \sigma_{\kappa_{G M}}$, and $\sigma_{\kappa_{d}}$. Right: Wind stress changes data in Pa, shown for June; other months are qualitatively similar. Bottom: resulting meridional velocity anomaly $\left(\mathrm{m} \mathrm{s}^{-1}\right)$ at $3700 \mathrm{~m}$. Positive values indicate a relative increase in northward velocity. 
The two sensitivity experiments show that multiple mechanisms suffice to improve the fit to extrapolated tracer distributions. This result is a symptom of the inability, characteristic of an underdetermined problem (see Chapter 2), of the observations to distinguish between more than one possible solution. That two quite different solutions (at least in terms of model parameter choices) arose from different choices in the prior uncertainties points to the important role of those values in the inverse problem. It is likely that other prior uncertainties could give rise to still more changes in the model that could fit the tracer observations.

That multiple solutions appear to fit the observations is not unexpected given the illposedness of the inverse problem. The fact that a dense distribution of hypothetical data with known boundary conditions was not able to distinguish between physical changes suggests that the picture may be even less clear using real data, where tracer end member gradients can be small and data are sparsely distributed and highly uncertain. Another caveat is that the processes leading to the formation of model AABW and NADW in the low-resolution model used are very different from the processes at work in the real ocean. For instance, water mass transformations leading to AABW formation via modification of high-salinity shelf water generated by brine rejection (believed to be the mechanism in the modern ocean) only begin to be resolved in the Southern Ocean State Estimate at 1/12 degree resolution (M. Mazloff, personal communication). For these reasons, the calculated sensitivities to control variables may be a strong function of the model's parameterized deep water formation.

The use of tracer extrapolation and AST must be handled with caution, particularly for simulations that are short relative to ocean equilibration times. To test whether control variable changes derived using asynchronous time stepping and extrapolation improve misfits in a forward simulation using synchronous time stepping, the model was integrated in two simulations, one under first-guess forcings and one including derived changes to $\kappa_{G M}$. After 600 years, the model-data misfit was not improved in the model including changes to $\kappa_{G M}$. Further analysis is necessary to determine whether this lack of improvement stems from in- 
complete model spin up, model drift, or biases in the inverse procedure due to AST and extrapolation. It is likely that model drift is to blame; there is no guarantee that changes in parameters that suffice to reduce model-data misfit over a 100-year interval will also reduce misfit in a much longer simulation. Beginning with an initial state that is equilibrated to first-guess forcings may mitigate this problem.

Previously suggested mechanisms to change the relative position of NADW and AABW during glacial periods include changes in abyssal stratification arising from to colder ocean temperatures [De Boer et al., 2007], changes in Southern Hemisphere westerly winds [Lauderdale et al., 2013], changes in the extent of Southern Ocean sea ice [Ferrari et al., 2014] and changes in North Atlantic salinity due to wind stress [Muglia and Schmittner, 2015]. The discussion in this chapter does not rule out the feasibility of those mechanisms, but rather adds to a growing list; perhaps what is most likely is that some combination of these and other influences contributed to observed features. More careful analysis of where abyssal mixing may have changed at the LGM - e.g., from changes in tides or stratification - will help evaluate the possibility that changes in turbulent transports could have played a major role in changing the LGM tracer distributions.

\subsection{Conclusions}

Many of the data available from past geological eras, including the LGM, comes from ocean tracers, but interpreting those data in terms of physical ocean processes has proven a challenging inverse problem, often yielding conflicting results. This chapter provides an answer to the question "What do paleo tracer data imply about the ocean circulation?" in the form of adjoint sensitivities of ocean forcing variables and internal mixing parameters (the control vector) to hypothetical tracer observations. This analysis required implementing two new approaches in the MITgcm adjoint, the use of vertically varying time steps in passive 
tracers and extrapolating to estimate tracer equilibria. In addition to paleoceanographic state estimation, these procedures could be used for any inverse problems where long time scales or tracers are implicated. For instance, using AST and tracer extrapolation for temperature and salinity in long simulations could be used to infer turbulent transport parameters in a quasi-steady simulation of the modern circulation. 


\section{Chapter 5}

\section{Conclusions and future work}

Our knowledge of the ocean in the geologic past comes both from paleoproxy data - collected from the seafloor, ice cores, and other natural archives - and from the physical relationships governing the motion of geophysical fluids as represented in ocean and climate models. This thesis uses models and data in concert to constrain paleoceanographic ocean circulations and paleoclimates, with an emphasis on proxy measurements of ocean tracers. The basis for all of the chapters is the least-squares fitting of ocean models to observations, which has the potential to yield best estimates constrained both by physics and data, to reveal shortcomings in models (towards their eventual improvement), and to extend the power and quantify the limitations of observations.

Chapter2 In Chapter 2, the modern ocean circulation was found to adequately fit $\delta^{18} \mathrm{O}_{c}$ time series from the last deglaciation by choice of mixed layer (ML) $\delta^{18} \mathrm{O}_{c}$ boundary conditions alone (no circulation changes were necessary). The strategy was to approximate ML $\delta^{18} \mathrm{O}_{c}$ from planktonic records, which record $\delta^{18} \mathrm{O}_{c}$ in the surface ocean, and benthic records, which record $\delta^{18} \mathrm{O}_{c}$ in the abyss. To estimate ML values from benthic records, I 
used an estimate of the modern circulation to backtrack tracer values from the deep ocean to the surface; disagreements between this benthic-derived $\mathrm{ML} \delta^{18} \mathrm{O}_{c}$ history and that inferred from planktonic records would indicate that some deviations from the modern circulation were necessary. Quantifying uncertainty in the reconstructions of $\mathrm{ML} \delta^{18} \mathrm{O}_{c}$ is necessary in order to make this comparison; it was found that the nullspace uncertainty in benthic reconstructions (due to the fact that most ML points do not communicate tracer signals to the abyss) limits this upstream-downstream approach using benthic and planktonic data as posed in that chapter. A prescription was made for record locations (at high latitudes near regions of deep water formation, where signals are filtered less by ocean mixing) that will be least affected by nullspace uncertainty. The framework established is a general one, and can be used with geochemical tracers other than $\delta^{18} \mathrm{O}_{c}$.

Future work on this problem has the potential to improve inference of circulation changes from passive tracer records and reconstructions of $\mathrm{ML} \delta^{18} \mathrm{O}_{c}$, which is an important goal in its own right, as it could inform whether ice sheets melted first in one hemisphere or the other. Solving for ML conditions weighted by their respective vertical fluxes to the abyss would make the inverse circulation problem better posed (fewer small singular values) and could be interpreted as inferring tracer end member values. Using information about how and why $\delta^{18} \mathrm{O}_{c}$ may have varied in the surface ocean could permit substantial progress on both fronts. $\mathrm{ML} \delta^{18} \mathrm{O}_{c}$ is set chiefly by atmospheric isotope cycling, upper ocean temperature, and river and glacial discharge (from meltwater, melting icebergs, etc.). Exactly how each of these processes may have been altered in the warming deglacial climate is a challenging problem in its own right, and lessons learned from data and models [Condron and Winsor, 2011, 2012, Caley et al., 2014, , Chapter 3 from this thesis] should be incorporated into future inverse work. For instance, we know that meltwater entering the ocean tends to flow along the coast in the direction of Kelvin wave propagation before gradually being entrained into ocean gyres. The spatial patterns resulting from likely outlets of meltwater (and their 
attendant time scales) could be taken as fingerprints of deglacial melting, and included in the inverse problem using column weighting. The effect would be both to reduce the space of unknowns and to make inferred $\mathrm{ML} \delta^{18} \mathrm{O}_{c}$ more physically comprehensible.

Chapter 3 Chapter 3 expands on the work of Dail [2012b] and Dail and Wunsch [2014] by constructing a glacial ocean state estimate - a fit of the MITgcm to the MARGO near-sea surface temperature data - derived by adjusting seasonal cycles of model atmospheric variables, initial conditions, and internal physical parameterizations. The fit to the data in the "current best estimate" is improved for all seasons (summer, winter, and the annual average) though sizable misfits to $\mathrm{SH}$ summer values remain at iteration 10 . The resulting simulation shows deviations from modern observations in the mean and spatial patterns of SST, sea ice extent, mixed layer depth, and circulation strength as measured by the barotropic streamfunction. Nevertheless, the changes diagnosed are not so large as to suggest major changes in the ocean's role in climate. An order 0 assessment of nullspace uncertainty (made by comparing two iterations with similar model-data misfits) suggests that the data poorly constrain flow strength in the deep ocean as diagnosed by the Atlantic Meridional Overturning Circulation and ocean heat transports.

Much work can still be done in quantifying and understanding features of the solution. Of particular interest is the Southern Ocean, where sea ice extent and changes in wind strength have been associated with changes in the deep circulation. Understanding why the model is slow to fit the data in that region could suggest problems in the model or in the data, which are nearly all of one type (diatoms). Changes in upper ocean heat transports and storage (e.g., thermocline depth) also warrant further investigation, as does the circulation under the perennially ice-covered Arctic.

Having a dynamically interpolated NSST reconstruction permits future work along sev- 
eral avenues. One might begin by combining inferred upper-ocean temperatures with planktonic measurements of $\delta^{18} \mathrm{O}_{c}$ to estimate surface $\delta^{18} \mathrm{O}_{w}$, which is locally correlated with salinity in the modern ocean [LeGrande and Schmidt, 2006] and could be instrumental in constraining AMOC rates if a paleo $\delta^{18} \mathrm{O}_{w}$-salinity relationship could be established. However, given that there are multiple ways for the model to fit the data, the implication is that there are multiple valid dynamical interpolations, and better quantification of adjoint solution uncertainties is essential. For instance, fitting the data by changing ocean heat transport driven by changes to large-scale wind stress would yield broader patterns of warming and cooling than would result from changes in air temperature and radiation, which can influence NSST locally. Solution NSST uncertainties should also reflect uncertainties in the data, which can be quite large. Progress is being made on formal adjoint uncertainty quantification [Kalmikov and Heimbach, 2014]; in the mean time, sensitivity experiments (for instance, obtaining solutions under different first guesses or prior control uncertainties) can be used to assess the robustness of solution features, as can comparing solutions at multiple iterations, as was done for $\mathrm{AMOC}$ reconstructions.

The goal for future state estimation at the LGM is to incorporate as much data as possible, including a wide diversity of data types. Here the major challenges lie in relating proxy observations to model variables and in adequately quantifying data uncertainties. From an adjoint modeling perspective, the issue of how to include new data types amounts to determining how an observation can be included in the cost function. In this work, it was straightforward to include NSST proxies in the cost function because ocean temperature is in the model state vector; by contrast, computing model-data misfits for $\delta^{18} \mathrm{O}_{c}$ necessitated including a new model state variable $\left(\delta^{18} \mathrm{O}_{c}\right)$ and approximating its equilibrium distribution. Substantial efforts (outlined in Chapter 4 ) were made towards fitting the model to these tracer observations; at the time of thesis completion, instabilities in the adjoint precluded their inclusion in the cost function. Implementing more complex proxy systems is likely to 
be even more involved. A first step is to adopt the same strategy used for $\delta^{18} \mathrm{O}_{c}$ and to compute the model-data misfit to get a sense of what can be learned. For instance, a first step in incorporating radiocarbon in the state estimate could be to include a radiocarbon-like tracer in the state vector and test whether any reservoirs of old abyssal waters [Marchitto et al. 2007, Stott et al., 2009, Burke and Robinson, 2012, Skinner et al., 2015] are simulated.

For new data to be used, descriptions of uncertainties alongside observational records must be publicly available and well documented, as they are in MARGO. Characterizing the residuals of least-squares fits to models may prove a useful post hoc assessment of these uncertainties. Many paleoceanographic data are affected by time uncertainty, which is problematic both for presumed steady intervals such as the LGM (because data from other geologic periods can erroneously presumed to be from the interval of interest) and especially in inference of transient intervals, where age errors can give rise to phase offsets and incorrect diagnoses of cause and effect. Development of statistical tools for handling this kind of uncertainty is important, as is the use of more precise dating techniques. Eventually, adopting Bayesian techniques or other approaches that can handle non-Gaussian uncertainties (as can arise from dating uncertainties) may be necessary.

The assumption of a seasonally steady circulation, while improving at face value how underconstrained the problem is by reducing the number of control variables and increasing the number of observations, biases the solution and may reduce the possible solution space. Errors in the assumption that the data represent a mean, quasi-steady state were not quantified, in part because glacial climate variability on interannual tand longer time scales is poorly known, and is likely to vary in space. Eventually, as more high-resolution records are developed over this interval, it will be useful to construct a time-varying LGM state estimate, both in order to reconstruct glacial climate variability and because transient tracer data have the potential to reveal more information about abyssal flows than do observations that are assumed steady, just as observations of a transient dye pulse transiting a pipe flow can reveal 
more about the flow than can observations downstream of a constant dye input.

The extent to which the adjoint solution is a "most likely" estimate of the LGM, rather than merely a curve-fitting exercise of a nonlinear model to data, is determined in part by how realistic the prior control uncertainties are. For instance, it is possible that a model fit to the data could be obtained by a 10,000-fold increase in the winds, but such a solution would be rejected as unphysical, and can be precluded by choosing the $\sigma$ values appropriately. More subtly, choices of these parameters can determine, e.g., whether the data are fit by changes in winds versus local heating, and can have a substantial effect on the final state, so that determining what likely typical excursions of the various controls were at the LGM is an important step for future state estimation work. Moreover, atmospheric controls were permitted to change independently in each $2^{\circ} \times 2^{\circ}$ model grid box, whereas observed length scales of these quantities are often much larger. Including information about spatiotemporal covariances of quantities like wind stress adjustments would reduce the number of effective unknown degrees of freedom and make control adjustments more readily relatable to atmospheric dynamics.

Chapter 4 Chapter 4 introduces two procedures to accelerate the equilibration of passive tracer distributions in ocean model. The first expands the approach of Bryan [1984] by generalizing the scheme for level-by-level variations in model time steps to vary in all three dimensions and showing that this is a form of Jacobi preconditioning. The second procedure shows that by integrating a "helper" tracer under specially chosen boundary conditions, a simple procedure exists to extrapolate to an estimate of equilibrium values based on an assumption of the arrival times of ventilating water masses. Derivation of these procedures was motivated by the particular necessities of using passive tracer measurements in the MITgcm state estimation framework, where integrating tracer distributions in adjoint simulations 
fully to equilibrium to compare to data is prohibitively computationally expensive.

These approaches were demonstrated in an experiment fitting the MITgcm to a hypothetical distribution of abyssal tracers intended to describe a shoaling of Antarctic Bottom Water, as has been hypothesized for the LGM based on several different data types. The adjoint procedure was able to improve the fit to the data over the integration period, and sensitivity tests suggest multiple possible mechanisms for explaining the observations. Future work will include investigating other possible mechanisms for shoaling AABW, looking at nonlinear sensitivities (by computing more than one iteration), and evaluating sensitivities over different integration lengths. Diagnosing which changes are most likely to have been responsible for shoaling LGM AABW will rely on careful consideration of the prior control uncertainties, as discussed in the previous section.

This type of idealized simulation, in which a relatively complex model (compared to the kinematic or geostrophic models typically used in abyssal inverse tracer problems) is fit to synthetic data, could be useful in future work by providing answers to the question "What might a change in ocean tracers imply about the ocean circulation?" For instance, in the context of the hypothetical deep ocean carbon reservoir described above, one could implement a radiocarbon-like tracer, construct synthetic data illustrating an isolated reservoir in the cost function, and use the adjoint to evaluate what changes (if any) could fit the data. If the data can be fit, then one could construct new, time-varying data illustrating a rapid ventilation of the isolated volume, again asking what ocean circulation changes could lead to this evolution.

Final thoughts A newcomer to the field might conclude upon reading this thesis that problems in paleoceanography are very difficult, even intractable, and that it is not clear where and how concrete progress (e.g., diagnosing abyssal circulation changes or the lack thereof 
with some high likelihood) can be made. Combining data and models makes it painfully clear just how underconstrained many of these problems are, in some cases with just a few hundreds of observations attempting to constrain thousands or millions of uncertain environmental variables. Perhaps for this reason, most of the work in paleoceanography and paleoclimate has a different tenor from that adopted here. Instead of posing an inverse problem, a typical procedure is to consider one or several records and suggest a hypothesis (often exciting) to explain them. This is useful for stretching our imagination about the possible range of climate states and climate transitions, but given how noisy the data are, it is often unclear how to test such hypotheses in a rigorous way. Left unchecked, there is a danger that some ideas may take on lives of their own and persist beyond their usefulness, similar to the notion of a light-bearing "luminiferous æther" that permeated the understanding of physics by the late 19 th century.

In light of the status quo, and despite the challenges, two important and promising roles for combining models and data in paleoclimatology are to investigate the boundaries of knowability and to identify and study those problems that do appear tractable. Somewhere between the statements that "the global mean surface ocean was colder at the LGM" and "the SST off the Galapagos on August 15 21,500 years ago was 24.7 degrees" lies a line between statements that are testable given current data and models, and those that are inspired by data but difficult to evaluate. Care must be taken when clarifying this boundary, as one can always find a model with enough unknown quantities that model-data inverse problems are underdetermined, potentially leading to an incorrect verdict that the data are insufficient. It is important to think carefully about the actual complexity of the system (how many degrees of freedom are there in the climate interval of interest?) and to use salient additional information whenever it exists. At the same time, caution is urged in not caricaturing the climate by oversimplifying the solution space, as can happen when the stated objective is to determine whether past climates were more or less "El Niño like" or when focusing too closely 
on AMOC strength without regard to structural changes in the circulation that might change what that number means. Such procedures have lurking priors and can impose unwanted structure and narrative on reconstructions of past climate. If a hypothesis appears nearly to be testable, then additional records can be suggested.

While there is no obvious recipe for identifying tractable problems, a sensible guideline is to look for proxy records that appear to have high signal-to-noise ratios. The LGM is a good example: its signature in paleoproxies is usually unambiguous, there is a relative abundance of data, and the climate excursion appears to have been large. Constraining the statistics of past events [e.g., Huybers and Curry, 2006] rather than actual historical sequences may be less sensitive to record uncertainty, though inferences of causality may be more difficult. Practitioners are urged to compare the degrees of freedom in data and models early on and not to shy from results that document data limitations, as they will guide future studies in these early days of paleoceanographic state estimation. 


\section{Bibliography}

Alistair Adcroft, Chris Hill, Jean-Michel Campin, John Marshall, and Patrick Heimbach. Overview of the formulation and numerics of the MIT GCM. In Proceedings of the ECMWF seminar series on Numerical Methods, Recent developments in numerical methods for atmosphere and ocean modelling, pages 139-149, 2004.

Jess F Adkins, Katherine McIntyre, and Daniel P Schrag. The salinity, temperature, and $\delta^{18} \mathrm{O}$ of the glacial deep ocean. Science, 298(5599):1769-1773, 2002.

R Alkama, M Kageyama, and G Ramstein. Freshwater discharges in a simulation of the Last Glacial Maximum climate using improved river routing. Geophysical research letters, 33 (21), 2006.

Daniel E. Amrhein. An inverse approach to to understanding deglacial benthic oxygen isotope records. Master's thesis, Massachusetts Institute of Technology / Woods Hole Oceanographic Inst., 2014.

Daniel E Amrhein, Geoffrey Gebbie, Olivier Marchal, and Carl Wunsch. Inferring surface water equilibrium calcite $\delta 180$ during the last deglacial period from benthic foraminiferal records: Implications for ocean circulation. Paleoceanography, 2015.

Maxime Ballarotta, L Brodeau, J Brandefelt, Peter Lundberg, and Kristofer Döös. Last Glacial Maximum world ocean simulations at eddy-permitting and coarse resolutions: do eddies contribute to a better consistency between models and palaeoproxies? Climate of the Past, 9:2669-2686, 2013.

Edouard Bard, Maurice Arnold, Pierre Maurice, Josette Duprat, Jean Moyes, and JeanClaude Duplessy. Retreat velocity of the North Atlantic polar front during the last deglaciation determined by ${ }^{14} \mathrm{C}$ accelerator mass spectrometry. Nature, 328(6133):791794, 1987.

Ann Bardin, François Primeau, and Keith Lindsay. An offline implicit solver for simulating prebomb radiocarbon. Ocean Modelling, 73:45-58, 2014.

S. Barker, P. Diz, M.J. Vautravers, J. Pike, G. Knorr, I.R. Hall, and W.S. Broecker. Interhemispheric Atlantic seesaw response during the last deglaciation. Nature, 457(7233): 1097-1102, 2009. 
Henning A Bauch, Helmut Erlenkeuser, Robert F Spielhagen, Ulrich Struck, Jens Matthiessen, Jörn Thiede, and Jan Heinemeier. A multiproxy reconstruction of the evolution of deep and surface waters in the subarctic Nordic seas over the last 30,000 yr. Quaternary Science Reviews, 20(4):659-678, 2001.

B.E. Bemis, H.J. Spero, J. Bijma, and D.W. Lea. Reevaluation of the oxygen isotopic composition of planktonic foraminifera: Experimental results and revised paleotemperature equations. Paleoceanography, 13(2):150-160, 1998.

Edward A Boyle and Lloyd D Keigwin. Deep circulation of the North Atlantic over the last 200,000 years: Geochemical evidence. Science, 218(4574):784-787, 1982.

P Braconnot, B Otto-Bliesner, M Kageyama, A Kitoh, A Laîné, M-F Loutre, O Marti, U Merkel, G Ramstein, P Valdes, et al. Results of PMIP2 coupled simulations of the Mid-Holocene and Last Glacial Maximum-Part 1: experiments and large-scale features. 2007.

Pascale Braconnot, Sandy P Harrison, Masa Kageyama, Patrick J Bartlein, Valerie MassonDelmotte, Ayako Abe-Ouchi, Bette Otto-Bliesner, and Yan Zhao. Evaluation of climate models using palaeoclimatic data. Nature Climate Change, 2(6):417-424, 2012.

Raymond S Bradley and John H England. The Younger Dryas and the sea of ancient ice. Quaternary Research, 70(1):1-10, 2008.

Esther C Brady, Bette L Otto-Bliesner, Jennifer E Kay, and Nan Rosenbloom. Sensitivity to glacial forcing in the CCSM4. Journal of Climate, 26(6):1901-1925, 2013.

Francis P Bretherton, Russ E Davis, and CB Fandry. A technique for objective analysis and design of oceanographic experiments applied to MODE-73. In Deep Sea Research and Oceanographic Abstracts, volume 23, pages 559-582. Elsevier, 1976.

C. Bronk Ramsey. Bayesian analysis of radiocarbon dates. Radiocarbon, 51(1):337-360, 2009.

Victor Brovkin, Andrey Ganopolski, David Archer, and Stefan Rahmstorf. Lowering of glacial atmospheric $\mathrm{CO} 2$ in response to changes in oceanic circulation and marine biogeochemistry. Paleoceanography, 22(4), 2007.

Kirk Bryan. Accelerating the convergence to equilibrium of ocean-climate models. Journal of Physical Oceanography, 14(4):666-673, 1984.

Harry L Bryden. New polynomials for thermal expansion, adiabatic temperature gradient and potential temperature of sea water. In Deep Sea Research and Oceanographic Abstracts, volume 20, pages 401-408. Elsevier, 1973.

Véronique Bugnion and Chris Hill. Equilibration mechanisms in an adjoint ocean general circulation model. Ocean Dynamics, 56(1):51-61, 2006. 
Andrea Burke and Laura F Robinson. The Southern Ocean's role in carbon exchange during the last deglaciation. Science, 335(6068):557-561, 2012.

Andrea Burke, Olivier Marchal, Louisa I Bradtmiller, Jerry F McManus, and Roger François. Application of an inverse method to interpret ${ }^{231} \mathrm{~Pa} /{ }^{230} \mathrm{Th}$ observations from marine sediments. Paleoceanography, 26(1), 2011.

T Caley, DM Roche, C Waelbroeck, and E Michel. Oxygen stable isotopes during the Last Glacial Maximum climate: perspectives from data-model (iLOVECLIM) comparison. Climate of the Past, 10(6):1939-1955, 2014.

Christopher D Charles, Philip N Froelich, Michael A Zibello, Richard A Mortlock, and Joseph J Morley. Biogenic opal in Southern Ocean sediments over the last 450,000 years: Implications for surface water chemistry and circulation. Paleoceanography, 6(6):697728, 1991.

P.U. Clark, J.D. Shakun, P.A. Baker, P.J. Bartlein, S. Brewer, E. Brook, A.E. Carlson, H. Cheng, D.S. Kaufman, Z. Liu, et al. Global climate evolution during the last deglaciation. Proceedings of the National Academy of Sciences, 109(19):E1134-E1142, 2012.

J Comiso. Bootstrap sea ice concentrations for NIMBUS-7 SMMR and DMSP SSM/I. Digital Media, National Snow and Ice Data Center, 1999.

A. Condron and P. Winsor. A subtropical fate awaited freshwater discharged from glacial Lake Agassiz. Geophysical Research Letters, 38(3):L03705, 2011.

Alan Condron and Peter Winsor. Meltwater routing and the Younger Dryas. Proceedings of the National Academy of Sciences, 109(49):19928-19933, 2012.

M Conkright, S Levitus, T O’Brien, T Boyer, J Antonov, and C Stephens. World Ocean Atlas 1998 CD-ROM data set documentation. National Oceanographic Data Center (NODC) Internal Report, Silver Spring, Maryland, 1998.

Margarita E Conkright, Syndey Levitus, and Timothy P Boyer. World Ocean Atlas: 1994 Nutrients, volume 1. NOAA Atlas NESDIS 1, 1994.

W Bo Curry and GP Lohmann. Carbon isotopic changes in benthic foraminifera from the western South Atlantic: Reconstruction of glacial abyssal circulation patterns. Quaternary Research, 18(2):218-235, 1982.

W.B. Curry and D.W. Oppo. Glacial water mass geometry and the distribution of $\delta^{13} \mathrm{C}$ of $\Sigma \mathrm{CO}_{2}$ in the western Atlantic Ocean. Paleoceanography, 20(1):PA1017, 2005.

W.B. Curry, J.C. Duplessy, L.D. Labeyrie, and N.J. Shackleton. Changes in the distribution of $\mathrm{d} 13 \mathrm{C}$ of deep water sigmaCO2 between the last glaciation and the Holocene. Paleoceanography, 3(3):317-341, 1988. 
Holly Dail. Atlantic Ocean circulation at the Last Glacial Maximum: Inferences from data and models. PhD thesis, MIT-WHOI Joint Program, 2012a.

Holly Dail. Atlantic Ocean circulation at the Last Glacial Maximum: Inferences from data and models. PhD thesis, MIT-WHOI Joint Program, 2012b.

Holly Dail and Carl Wunsch. Dynamical Reconstruction of Upper-Ocean Conditions in the Last Glacial Maximum Atlantic. Journal of Climate, 27(2):807-823, 2014.

Gokhan Danabasoglu. A comparison of global ocean general circulation model solutions obtained with synchronous and accelerated integration methods. Ocean Modelling, 7(3): 323-341, 2004.

Gokhan Danabasoglu, James C McWilliams, and William G Large. Approach to equilibrium in accelerated global oceanic models. Journal of Climate, 9(5):1092-1110, 1996.

AM De Boer, DM Sigman, JR Toggweiler, and JL Russell. Effect of global ocean temperature change on deep ocean ventilation. Paleoceanography, 22(2), 2007.

A De Vernal, A Rosell-Melé, M Kucera, C Hillaire-Marcel, F Eynaud, M Weinelt, T Dokken, and M Kageyama. Comparing proxies for the reconstruction of LGM sea-surface conditions in the northern North Atlantic. Quaternary Science Reviews, 25(21):2820-2834, 2006.

DP Dee, SM Uppala, AJ Simmons, Paul Berrisford, P Poli, S Kobayashi, U Andrae, MA Balmaseda, G Balsamo, P Bauer, et al. The ERA-Interim reanalysis: Configuration and performance of the data assimilation system. Quarterly Journal of the royal meteorological society, 137(656):553-597, 2011.

Thomas L Delworth and Michael E Mann. Observed and simulated multidecadal variability in the Northern Hemisphere. Climate Dynamics, 16(9):661-676, 2000.

Trond M Dokken and Eystein Jansen. Rapid changes in the mechanism of ocean convection during the last glacial period. Nature, 401(6752):458-461, 1999.

JC Duplessy, NJ Shackleton, RG Fairbanks, L Labeyrie, D Oppo, and N Kallel. Deepwater source variations during the last climatic cycle and their impact on the global deepwater circulation. Paleoceanography, 3(3):343-360, 1988.

I. Eisenman, C.M. Bitz, and E. Tziperman. Rain driven by receding ice sheets as a cause of past climate change. Paleoceanography, 24(4):PA4209, 2009.

Balázs M Fekete, Charles J Vörösmarty, and Wolfgang Grabs. High-resolution fields of global runoff combining observed river discharge and simulated water balances. Global Biogeochemical Cycles, 16(3), 2002. 
Raffaele Ferrari, Malte F Jansen, Jess F Adkins, Andrea Burke, Andrew L Stewart, and Andrew F Thompson. Antarctic sea ice control on ocean circulation in present and glacial climates. Proceedings of the National Academy of Sciences, 111(24):8753-8758, 2014.

P Fofonoff and RC Millard. Algorithms for computation of fundamental properties of seawater. UNESCO technical papers in marine science, 1983.

Gaël Forget. Mapping ocean observations in a dynamical framework: A 2004-06 ocean atlas. Journal of Physical Oceanography, 40(6):1201-1221, 2010.

GAEL Forget, J-M Campin, P Heimbach, CN Hill, RM Ponte, and C Wunsch. ECCO version 4: an integrated framework for non-linear inverse modeling and global ocean state estimation. 2015a.

Gaël Forget, David Ferreira, and Xingfeng Liang. On the observability of turbulent transport rates by Argo: supporting evidence from an inversion experiment. Ocean Science, 11(5): 839, $2015 b$.

Alexandre Ganachaud and Carl Wunsch. Improved estimates of global ocean circulation, heat transport and mixing from hydrographic data. Nature, 408(6811):453-457, 2000.

G. Gebbie. Tracer transport timescales and the observed Atlantic-Pacific lag in the timing of the Last Termination. Paleoceanography, 27(3):PA3225, 2012.

G. Gebbie and P. Huybers. Total matrix intercomparison: a method for resolving the geometry of water-mass pathways. Journal of Physical Oceanography, 40(8):1710-1728, 2010.

G. Gebbie and P. Huybers. How is the ocean filled? Geophysical Research Letters, 38(6): L06604, 2011.

G. Gebbie and P. Huybers. The mean age of ocean waters inferred from radiocarbon observations: sensitivity to surface sources and accounting for mixing histories. Journal of Physical Oceanography, 42(2):291-305, 2012.

Geoffrey Gebbie. How much did Glacial North Atlantic Water shoal? Paleoceanography, 29(3):190-209, 2014.

Geoffrey Gebbie and Peter Huybers. Meridional circulation during the Last Glacial Maximum explored through a combination of South Atlantic $\delta^{18} \mathrm{O}$ observations and a geostrophic inverse model. Geochemistry Geophysics Geosystems, 7(11):Q11N07, 2006.

Geoffrey A Gebbie. Subduction in an eddy-resolving state estimate of the northeast Atlantic Ocean. PhD thesis, Massachusetts Institute of Technology and Woods Hole Oceanographic Institution, 2004. 
Peter R Gent and James C McWilliams. Isopycnal mixing in ocean circulation models. Journal of Physical Oceanography, 20(1):150-155, 1990.

Peter R Gent, Gokhan Danabasoglu, Leo J Donner, Marika M Holland, Elizabeth C Hunke, Steve R Jayne, David M Lawrence, Richard B Neale, Philip J Rasch, Mariana Vertenstein, et al. The community climate system model version 4. Journal of Climate, 24(19):49734991, 2011.

Ralf Giering and Thomas Kaminski. Recipes for adjoint code construction. ACM Transactions on Mathematical Software (TOMS), 24(4):437-474, 1998.

Jean Charles Gilbert and Claude Lemaréchal. Some numerical experiments with variablestorage quasi-Newton algorithms. Mathematical programming, 45(1-3):407-435, 1989.

Ada Gjermundsen and J. H. LaCasce. Comparing the linear and nonlinear buoyancy-driven circulation. Tellus, submitted.

Anand Gnanadesikan. A simple predictive model for the structure of the oceanic pycnocline. Science, 283(5410):2077-2079, 1999.

Viktor Gouretski and Klaus Peter Koltermann. WOCE global hydrographic climatology. Berichte des BSH, 35:1-52, 2004.

JC Hargreaves, A Paul, R Ohgaito, A Abe-Ouchi, and JD Annan. Are paleoclimate model ensembles consistent with the MARGO data synthesis? Climate of the Past, 7(3):917933, 2011.

Christopher T Hayes, Robert F Anderson, Martin Q Fleisher, Kuo-Fang Huang, Laura F Robinson, Yanbin Lu, Hai Cheng, R Lawrence Edwards, and S Bradley Moran. 230 Th and $231 \mathrm{~Pa}$ on GEOTRACES GA03, the US GEOTRACES North Atlantic transect, and implications for modern and paleoceanographic chemical fluxes. Deep Sea Research Part II: Topical Studies in Oceanography, 116:29-41, 2015.

JD Hays, J. Imbrie, and NJ Shackleton. Variations in the Earth's Orbit: Pacemaker of the Ice Ages. Science, 194:1121-1134, 1976.

Matthew W Hecht. Forward-in-time upwind-weighted methods in ocean modelling. International journal for numerical methods in fluids, 50(10):1159-1173, 2006.

David A Hodell, Christopher D Charles, Jason H Curtis, P Graham Mortyn, Ulysses S Ninnemann, and Kathryn A Venz. Data report: Oxygen isotope stratigraphy of ODP Leg 177 Sites 1088, 1089, 1090, 1093, and 1094. In Proc. Ocean Drill. Program Sci. Results, volume 177, pages 1-26, 2003.

Aixue Hu, Gerald A Meehl, Bette L Otto-Bliesner, Claire Waelbroeck, Weiqing Han, MarieFrance Loutre, Kurt Lambeck, Jerry X Mitrovica, and Nan Rosenbloom. Influence of Bering Strait flow and North Atlantic circulation on glacial sea-level changes. Nature Geoscience, 3(2):118-121, 2010. 
T Hughes, GH Denton, and MG Grosswald. Was there a late-Wiirm Arctic ice sheet. Nature, 266:596-602, 1977.

M. Hüls and R. Zahn. Millennial-scale sea surface temperature variability in the western tropical North Atlantic from planktonic foraminiferal census counts. Paleoceanography, 15(6):659-678, 2000.

Peter Huybers and William Curry. Links between annual, Milankovitch and continuum temperature variability. Nature, 441(7091):329-332, 2006.

Peter Huybers, Geoffrey Gebbie, and Olivier Marchal. Can paleoceanographic tracers constrain meridional circulation rates? Journal of physical oceanography, 37(2):394-407, 2007.

T.S. Ivanochko, R.S. Ganeshram, G.J.A. Brummer, G. Ganssen, S.J.A. Jung, S.G. Moreton, and D. Kroon. Variations in tropical convection as an amplifier of global climate change at the millennial scale. Earth and Planetary Science Letters, 235(1-2):302-314, 2005.

Gregory C Johnson. Quantifying Antarctic bottom water and North Atlantic deep water volumes. Journal of Geophysical Research: Oceans (1978-2012), 113(C5), 2008.

SJA Jung, GM Ganssen, and GR Davies. Multidecadal variations in the early Holocene outflow of Red Sea Water into the Arabian Sea. Paleoceanography, 16(6):658-668, 2001.

S.J.A. Jung, D. Kroon, G. Ganssen, F. Peeters, and R. Ganeshram. Enhanced Arabian Sea intermediate water flow during glacial North Atlantic cold phases. Earth and Planetary Science Letters, 280(1-4):220-228, 2009.

Alexander G Kalmikov and Patrick Heimbach. A Hessian-based method for uncertainty quantification in global ocean state estimation. SIAM Journal on Scientific Computing, 36 (5):S267-S295, 2014.

A Birol Kara, Peter A Rochford, and Harley E Hurlburt. An optimal definition for ocean mixed layer depth. Technical report, DTIC Document, 2000.

LD Keigwin and MA Schlegel. Ocean ventilation and sedimentation since the glacial maximum at $3 \mathrm{~km}$ in the western North Atlantic. Geochemistry, Geophysics, Geosystems, 3 (6):1-14, 2002.

Lloyd D Keigwin. Radiocarbon and stable isotope constraints on Last Glacial Maximum and Younger Dryas ventilation in the western North Atlantic. Paleoceanography, 19(4), 2004.

Robert M Key, Alex Kozyr, Chris L Sabine, Kitack Lee, Rik Wanninkhof, John L Bullister, Richard A Feely, Frank J Millero, Calvin Mordy, and T-H Peng. A global ocean carbon climatology: Results from Global Data Analysis Project (GLODAP). Global Biogeochemical Cycles, 18(4), 2004. 
Samar Khatiwala. A computational framework for simulation of biogeochemical tracers in the ocean. Global Biogeochemical Cycles, 21(3):GB3001, 2007.

Samar Khatiwala. Fast spin up of ocean biogeochemical models using matrix-free NewtonKrylov. Ocean Modelling, 23(3):121-129, 2008.

Samar Khatiwala, Martin Visbeck, and Mark A Cane. Accelerated simulation of passive tracers in ocean circulation models. Ocean Modelling, 9(1):51-69, 2005.

Till Kuhlbrodt, Alexa Griesel, Marisa Montoya, Anders Levermann, Matthias Hofmann, and Stefan Rahmstorf. On the driving processes of the Atlantic meridional overturning circulation. Reviews of Geophysics, 45(2), 2007.

T Kurahashi-Nakamura, M Losch, and A Paul. Can sparse proxy data constrain the strength of the Atlantic meridional overturning circulation? Geoscientific Model Development Discussions, 6(3):4417-4445, 2013.

Monique Labracherie, Laurent D Labeyrie, Josette Duprat, Edouard Bard, Maurice Arnold, Jean-Jacques Pichon, and Jean-Claude Duplessy. The last deglaciation in the Southern Ocean. Paleoceanography, 4(6):629-638, 1989.

William George Large and Stephen G Yeager. Diurnal to decadal global forcing for ocean and sea-ice models: the data sets and flux climatologies. National Center for Atmospheric Research Boulder, 2004.

Jonathan M Lauderdale, Alberto C Naveira Garabato, Kevin IC Oliver, Michael J Follows, and Richard G Williams. Wind-driven changes in Southern Ocean residual circulation, ocean carbon reservoirs and atmospheric CO2. Climate dynamics, 41(7-8):2145-2164, 2013.

P. LeGrand and C. Wunsch. Constraints from paleotracer data on the North Atlantic circulation during the last glacial maximum. Paleoceanography, 10(6):1011-1045, 1995.

A.N. LeGrande and G.A. Schmidt. Global gridded data set of the oxygen isotopic composition in seawater. Geophysical Research Letters, 33(12):L12604, 2006.

Xingwen Li and François W Primeau. A fast Newton-Krylov solver for seasonally varying global ocean biogeochemistry models. Ocean Modelling, 23(1):13-20, 2008.

M. Little, RR. Schneider, D. Kroon, T. Price, T. Bickert, and G. Weger. Rapid palaeoceanographic changes in the Benguela Upwelling System for the last 160,000 years as indicated by abundances of planktonic foraminifer. Paleogeography, Palaeoclimatology, Palaeoecology, 130:135-161, 1997.

Z. Liu, BL Otto-Bliesner, F. He, EC Brady, R. Tomas, PU Clark, AE Carlson, J. LynchStieglitz, W. Curry, E. Brook, et al. Transient simulation of last deglaciation with a new mechanism for Bølling-Allerød warming. Science, 325(5938):310-314, 2009. 
Martin Losch, Dimitris Menemenlis, Jean-Michel Campin, Patick Heimbach, and Chris Hill. On the formulation of sea-ice models. Part 1: Effects of different solver implementations and parameterizations. Ocean Modelling, 33(1):129-144, 2010.

DC Lund, JF Adkins, and R. Ferrari. Abyssal Atlantic circulation during the Last Glacial Maximum: Constraining the ratio between transport and vertical mixing. Paleoceanography, 26(1):PA1213, 2011.

J. Lynch-Stieglitz, W.B. Curry, and N. Slowey. Weaker Gulf Stream in the Florida straits during the last glacial maximum. Nature, 402(6762):644-648, 1999.

Jean Lynch-Stieglitz, Jess F Adkins, William B Curry, Trond Dokken, Ian R Hall, Juan Carlos Herguera, Joël J-M Hirschi, Elena V Ivanova, Catherine Kissel, Olivier Marchal, et al. Atlantic meridional overturning circulation during the Last Glacial Maximum. science, 316(5821):66-69, 2007.

S. Manabe and RJ Stouffer. Two stable equilibria of a coupled ocean-atmosphere model. Journal of Climate, 1(9):841-866, 1988.

Olivier Marchal and William B Curry. On the abyssal circulation in the glacial Atlantic. Journal of Physical Oceanography, 38(9):2014-2037, 2008.

Olivier Marchal, Claire Waelbroeck, and Alain Colin de Verdière. On the Movements of the North Atlantic Subpolar Front in the Preinstrumental Past*. Journal of Climate, 29(4): 1545-1571, 2016.

Thomas M Marchitto and Wallace S Broecker. Deep water mass geometry in the glacial Atlantic Ocean: A review of constraints from the paleonutrient proxy $\mathrm{Cd} / \mathrm{Ca}$. Geochemistry, Geophysics, Geosystems, 7(12), 2006.

Thomas M Marchitto, Delia W Oppo, and William B Curry. Paired benthic foraminiferal $\mathrm{Cd} / \mathrm{Ca}$ and $\mathrm{Zn} / \mathrm{Ca}$ evidence for a greatly increased presence of Southern Ocean Water in the glacial North Atlantic. Paleoceanography, 17(3):10-1, 2002.

Thomas M Marchitto, Scott J Lehman, Joseph D Ortiz, Jacqueline Flückiger, and Alexander van Geen. Marine radiocarbon evidence for the mechanism of deglacial atmospheric CO2 rise. science, 316(5830):1456-1459, 2007.

John Marshall, Alistair Adcroft, Chris Hill, Lev Perelman, and Curt Heisey. A finite-volume, incompressible Navier Stokes model for studies of the ocean on parallel computers. Journal of Geophysical Research: Oceans (1978-2012), 102(C3):5753-5766, 1997.

Trevor J McDougall and Paul M Barker. Getting started with TEOS-10 and the Gibbs Seawater (GSW) oceanographic toolbox. SCOR/IAPSO WG, 127:1-28, 2011. 
David McGee, Aaron Donohoe, John Marshall, and David Ferreira. Changes in ITCZ location and cross-equatorial heat transport at the Last Glacial Maximum, Heinrich Stadial 1, and the mid-Holocene. Earth and Planetary Science Letters, 390:69-79, 2014.

JF McManus, R. Francois, J.M. Gherardi, LD Keigwin, and S. Brown-Leger. Collapse and rapid resumption of Atlantic meridional circulation linked to deglacial climate changes. Nature, 428(6985):834-837, 2004.

Madeline D Miller, Mark Simons, Jess F Adkins, and Sarah E Minson. The Information Content of Pore Fluid $\delta 18 \mathrm{O}$ and [Cl-]. Journal of Physical Oceanography, 45(8):20702094, 2015.

MD Miller, JF Adkins, D Menemenlis, and MP Schodlok. The role of ocean cooling in setting glacial southern source bottom water salinity. Paleoceanography, 27(3), 2012.

Christian Millo, Michael Sarnthein, Antje Voelker, and Helmut Erlenkeuser. Variability of the Denmark Strait overflow during the last glacial maximum. Boreas, 35(1):50-60, 2006.

Juan Muglia and Andreas Schmittner. Glacial Atlantic overturning increased by wind stress in climate models. Geophysical Research Letters, 42(22):9862-9868, 2015.

CS Nelson, IL Hendy, HL Neil, CH Hendy, and PPE Weaver. Last glacial jetting of cold waters through the Subtropical Convergence zone in the Southwest Pacific off eastern New Zealand, and some geological implications. Palaeogeography, Palaeoclimatology, Palaeoecology, 156(1):103-121, 2000.

Kevin IC Oliver, Babette AA Hoogakker, S Crowhurst, GM Henderson, REM Rickaby, NR Edwards, and H Elderfield. A synthesis of marine sediment core $\delta 13 \mathrm{C}$ data over the last 150000 years. Climate of the Past, 6:645-673, 2010.

Delia W Oppo, William B Curry, and Jerry F McManus. What do benthic $\delta 13 \mathrm{C}$ and $\delta 18 \mathrm{O}$ data tell us about Atlantic circulation during Heinrich Stadial 1? Paleoceanography, 30 (4):353-368, 2015.

DW Oppo and SJ Lehman. Mid-depth circulation of the subpolar North Atlantic during the last glacial maximum. Science, 259(5098):1148-1152, 1993.

DR Ostermann and WB Curry. Calibration of stable isotopic data: an enriched $\delta 180$ standard used for source gas mixing detection and correction. Paleoceanography, 15(3):353360, 2000.

BL Otto-Bliesner, CD Hewitt, TM Marchitto, E Brady, A Abe-Ouchi, M Crucifix, S Murakami, and SL Weber. Last Glacial Maximum ocean thermohaline circulation: PMIP2 model intercomparisons and data constraints. Geophysical Research Letters, 34(12), 2007.

S. Peacock and M. Maltrud. Transit-time distributions in a global ocean model. Journal of Physical Oceanography, 36(3):474-495, 2006. 
WR Peltier. Global glacial isostasy and the surface of the ice-age Earth: The ICE-5G(VM2) model and GRACE. Annu. Rev. Earth Planet. Sci., 32:111-149, 2004.

Jean-Jacques Pichon, Laurent D Labeyrie, Gilles Bareille, Monique Labracherie, Josette Duprat, and Jean Jouzel. Surface water temperature changes in the high latitudes of the southern hemisphere over the Last Glacial-Interglacial Cycle. Paleoceanography, 7(3): 289-318, 1992.

Helen R Pillar, Patrick Heimbach, Helen L Johnson, and David P Marshall. Dynamical attribution of recent variability in Atlantic overturning. Journal of Climate, 29(9):33393352, 2016.

Rudolph W Preisendorfer and Curtis D Mobley. Principal component analysis in meteorology and oceanography, volume 425. Elsevier Amsterdam, 1988.

William H Press, George B Rybicki, and Jacqueline N Hewitt. The time delay of gravitational lens 0957+ 561. I-Methodology and analysis of optical photometric data. IIAnalysis of radio data and combined optical-radio analysis. The Astrophysical Journal, 385:404-420, 1992.

Martha H Redi. Oceanic isopycnal mixing by coordinate rotation. Journal of Physical Oceanography, 12(10):1154-1158, 1982.

PJ Reimer, MGL Baillie, E. Bard, A. Bayliss, JW Beck, PG Blackwell, C.B. Ramsey, CE Buck, GS Burr, RL Edwards, et al. INTCAL09 and MARINE09 radiocarbon age calibration curves, 0-50,000 years calendar BP. Radiocarbon, 51(4):1111-1150, 2009.

Stephany I Rubin and Robert M Key. Separating natural and bomb-produced radiocarbon in the ocean: The potential alkalinity method. Global Biogeochemical Cycles, 16(4):52-1, 2002 .

C. Rühlemann, S. Mulitza, P.J. Muller, G. Wefer, and R. Zahn. Warming of the tropical Atlantic Ocean and slowdown of thermohaline circulation during the last deglaciation. Nature, 402(6761):511-514, 1999.

R.L. Rutberg and S.L. Peacock. High-latitude forcing of interior ocean $\delta 13 C$. Paleoceanography, 21(2):PA2012, 2006.

George B Rybicki and William H Press. Interpolation, realization, and reconstruction of noisy, irregularly sampled data. The Astrophysical Journal, 398:169-176, 1992.

Peter M Saunders. Practical conversion of pressure to depth. Journal of Physical Oceanography, 11:573-578, 1981.

A Schmittner, JAM Green, and S-B Wilmes. Glacial ocean overturning intensified by tidal mixing in a global circulation model. Geophysical Research Letters, 42(10):4014-4022, 2015. 
Joachim Schönfeld, Rainer Zahn, and Lúcia de Abreu. Surface and deep water response to rapid climate changes at the Western Iberian Margin. Global and Planetary Change, 36 (4):237-264, 2003.

NJ Shackleton, J-C Duplessy, M Arnold, P Maurice, MA Hall, and J Cartlidge. Radiocarbon age of last glacial Pacific deep water. Nature, 335(6192):708-711, 1988.

NJ Shackleton, RG Fairbanks, Tzu-chien Chiu, and F Parrenin. Absolute calibration of the Greenland time scale: implications for Antarctic time scales and for Delta ${ }^{14}$ C. Quaternary Science Reviews, 23(14):1513-1522, 2004.

J.D. Shakun, P.U. Clark, F. He, S.A. Marcott, A.C. Mix, Z. Liu, B. Otto-Bliesner, A. Schmittner, and E. Bard. Global warming preceded by increasing carbon dioxide concentrations during the last deglaciation. Nature, 484(7392):49-54, 2012.

L Skinner, IN McCave, Lionel Carter, Stewart Fallon, AE Scrivner, and F Primeau. Reduced ventilation and enhanced magnitude of the deep Pacific carbon pool during the last glacial period. Earth and Planetary Science Letters, 411:45-52, 2015.

LC Skinner and NJ Shackleton. Rapid transient changes in northeast Atlantic deep water ventilation age across Termination I. Paleoceanography, 19(2):PA2005, 2004.

LC Skinner and NJ Shackleton. An Atlantic lead over Pacific deep-water change across Termination I: implications for the application of the marine isotope stage stratigaphy. Quaternary Science Reviews, 24:571-580, 2005.

LC Skinner, NJ Shackleton, and H. Elderfield. Millennial-scale variability of deep-water temperature and $\delta^{18} \mathrm{O}_{\mathrm{dw}}$ indicating deep-water source variations in the Northeast Atlantic, 0-34 cal. ka BP. Geochemistry Geophysics Geosystems, 4(12):1098, 2003.

LC Skinner, S. Fallon, C. Waelbroeck, E. Michel, and S. Barker. Ventilation of the deep Southern Ocean and deglacial $\mathrm{CO}_{2}$ rise. Science, 328(5982):1147, 2010.

Walter HF Smith and David T Sandwell. Global sea floor topography from satellite altimetry and ship depth soundings. Science, 277(5334):1956-1962, 1997.

Lowell Stott, John Southon, Axel Timmermann, and Athanasios Koutavas. Radiocarbon age anomaly at intermediate water depth in the Pacific Ocean during the last deglaciation. Paleoceanography, 24(2), 2009.

Gilbert Strang and Kaija Aarikka. Introduction to applied mathematics, volume 16. Wellesley-Cambridge Press Wellesley, MA, 1986.

Lynne D Talley, Joseph L Reid, and Paul E Robbins. Data-based meridional overturning streamfunctions for the global ocean. Journal of Climate, 16(19):3213-3226, 2003. 
Wang Tao, Liu Yi, and Huang Wei. Last glacial maximum sea surface temperatures: A model-data comparison. Atmospheric and Oceanic Science Letters, 6(5):233-239, 2013.

Kevin E Trenberth, Jerry G Olson, and William George Large. A global ocean wind stress climatology based on ECMWF analyses. Climate and Global Dynamics Division, National Center for Atmospheric Research, 1989.

Eli Turkel. Review of preconditioning methods for fluid dynamics. Applied Numerical Mathematics, 12(1):257-284, 1993.

L. Vidal, RR Schneider, O. Marchal, T. Bickert, TF Stocker, and G. Wefer. Link between the North and South Atlantic during the Heinrich events of the last glacial period. Climate Dynamics, 15(12):909-919, 1999.

C. Waelbroeck, C. Levi, JC Duplessy, L. Labeyrie, E. Michel, E. Cortijo, F. Bassinot, and F. Guichard. Distant origin of circulation changes in the Indian Ocean during the last deglaciation. Earth and Planetary Science Letters, 243(1-2):244-251, 2006.

C. Waelbroeck, LC Skinner, L. Labeyrie, J.C. Duplessy, E. Michel, N.V. Riveiros, J.M. Gherardi, and F. Dewilde. The timing of deglacial circulation changes in the Atlantic. Paleoceanography, 26(3):PA3213, 2011.

Claere Waelbroeck, A Paul, Michael Kucera, A Rosell-Melé, Mara Weinelt, Ralph Schneider, AC Mix, A Abelmann, L Armand, E Bard, et al. Constraints on the magnitude and patterns of ocean cooling at the Last Glacial Maximum. Nature Geoscience, 2(2):127$132,2009$.

Dailin Wang. A note on using the accelerated convergence method in climate models. Tellus A, 53(1):27-34, 2001.

Anthony Weaver and Philippe Courtier. Correlation modelling on the sphere using a generalized diffusion equation. Quarterly Journal of the Royal Meteorological Society, 127 (575):1815-1846, 2001.

Wilford F Weeks and Stephen F Ackley. The growth, structure, and properties of sea ice. In The geophysics of sea ice, pages 9-164. Springer, 1986.

A. Winguth, D. Archer, E. Maier-Reimer, and U. Mikolajewicz. Paleonutrient data analysis of the glacial Atlantic using an adjoint ocean general circulation model. Inverse Methods in Global Biogeochemical Cycles. Geophys. Monogr., 114:171-183, 2000.

C. Wunsch. Determining paleoceanographic circulations, with emphasis on the Last Glacial Maximum. Quaternary Science Reviews, 22(2):371-385, 2003.

C. Wunsch. Discrete inverse and state estimation problems: with geophysical fluid applications. Cambridge Univ Pr, 2006. 
C. Wunsch and P. Heimbach. How long to oceanic tracer and proxy equilibrium? Quaternary Science Reviews, 27(7-8):637-651, 2008.

Carl Wunsch. Last Glacial Maximum and deglacial abyssal seawater oxygen isotopic ratios. Climate of the Past, 12(6):1281-1296, 2016.

Carl Wunsch and Patrick Heimbach. Practical global oceanic state estimation. Physica D: Nonlinear Phenomena, 230(1):197-208, 2007.

Carl Wunsch and Patrick Heimbach. Two Decades of the Atlantic Meridional Overturning Circulation: Anatomy, Variations, Extremes, Prediction, and Overcoming Its Limitations. Journal of Climate, (2013), 2013.

R. Zahn and A. Stüber. Suborbital intermediate water variability inferred from paired benthic foraminiferal $\mathrm{Cd} / \mathrm{Ca}$ and $\delta^{13} \mathrm{C}$ in the tropical West Atlantic and linking with North Atlantic climates. Earth and Planetary Science Letters, 200(1-2):191-205, 2002.

M. Zarriess and A. Mackensen. The tropical rainbelt and productivity changes off northwest Africa: a 31,000-year high-resolution record. Marine Micropaleontology, 76(3-4):76-91, 2010.

M. Zarriess and A. Mackensen. Testing the impact of seasonal phytodetritus deposition on $\delta 13 \mathrm{C}$ of epibenthic foraminifer Cibicidoides wuellerstorfi: A 31,000 year high-resolution record from the northwest African continental slope. Paleoceanography, 26(2):PA2202, 2011.

Xu Zhang, Gerrit Lohmann, Gregor Knorr, and Xu Xu. Different ocean states and transient characteristics in Last Glacial Maximum simulations and implications for deglaciation. Climate of the Past, 9:2319-2333, 2013.

Ning Zhao, Olivier Marchal, Lloyd D Keigwin, Daniel E Amrhein, and Geoffrey Gebbie. A synthesis of deglacial deep-sea radiocarbon records and a test of their consistency with modern ocean circulation. Quaternary Sci, in prep. 